$25-2983$

$I-11348$

$x^{e-4}$

ESTIMATES OF INTERNAL

DOSE EQUIVALENT FROM

INHALATION AND

INGESTION OF SELECTED

RADIONUCLIDES 


\section{DISCLAIMER}

This report was prepared as an account of work sponsored by an agency of the United States Government. Neither the United States Government nor any agency Thereof, nor any of their employees, makes any warranty, express or implied, or assumes any legal liability or responsibility for the accuracy, completeness, or usefulness of any information, apparatus, product, or process disclosed, or represents that its use would not infringe privately owned rights. Reference herein to any specific commercial product, process, or service by trade name, trademark, manufacturer, or otherwise does not necessarily constitute or imply its endorsement, recommendation, or favoring by the United States Government or any agency thereof. The views and opinions of authors expressed herein do not necessarily state or reflect those of the United States Government or any agency thereof. 


\section{DISCLAIMER}

Portions of this document may be illegible in electronic image products. Images are produced from the best available original document. 


\section{NOTICE}

This report was prepared as an account of work sponsored by the United States Government. Neither the United States nor the Department of Energy, nor any of their employees, nor any of their contractors, subcontractors, or their employees, makes any warranty, expressed or implied, or assumes any legal liability or responsibility for the accuracy, completeness, or usefulness of any information, apparatus, product, or process disclosed, or represents that its use would not infringe privately owned rights.

\section{Printed in the United States of America}

Available from

National Technical Information Service

U.S. Department of Commerce

5285 Port Royal Road

Springfield, VA 22161

NTIS price codes

Printed copy: A05

Microfiche copy: A01

\section{TECHNICAL SUPPORT CONTRACT}

DE-AC04-78ALO5346

WESTINGHOUSE ELECTRIC CORP.

D'APPOLONIA CONSULTING ENGRS.

DRAVO ENGINEERS, INC.

GIBBS \& HILL, INC.

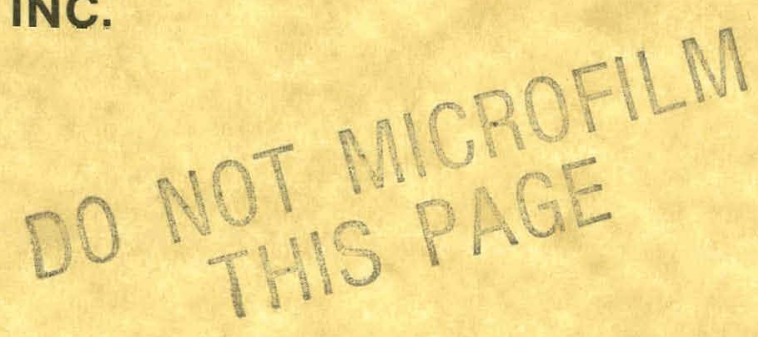




\title{
ESTIMATES OF INTERNAL-DOSE EQUIVALENT \\ FROM INHALATION AND INGESTION \\ OF SELECTED RADIONUCLIDES
}

WIPP-DOE- -176

DE83 017998

\author{
Donald E. Dunning \\ Evaluation Research Corporation \\ 800 Oak Ridge Turnpike, Sulte 501 \\ Oak Ridge, TN 37830
}

\section{DISCLAIMER}

This report was prepared as an account of work sponsored by an agency of the United States Government. Neither the United States Government nor any agency thereof, nor any of their employees, makes any warranty, express or implied, or assumes any legal liability or responsibility for the accuracy, completeness, or usefulness of any information, apparatus, product, or process disclosed, or represents that its use would not infringe privately owned rights. Reference herein to any specific commercial product, process, or service by trade name, trademark, manufacturer, or otherwise does not necessarily constitute or imply its endursement, recommendation, or favoring by the United States Government or any agency thereof. The views and opinions of authors expressed herein do not necessarily state or reflect those of the United States Government or any agency thereof.

\section{NOTICE}

POETHONS OF THIS REPBRT ARE ILLEGIBLE.

Prepared for

It has been reproduced from the best available copy to permit the broadest possible availability.

\author{
Westinghouse Electric Corporation \\ Advanced Energy Systems Division \\ W.I.P.P. Project \\ P.0. Box 40039
}

2501 Yale Blvd., S.E.

Albuquerque, NM 87196 
11

Table of Contents

Page

ABSTRACT . . . . . . . . . . . . . . . .

INTRODUCTION

REFERENCES

APPENDIX I - ESTIMATES OF 50 -YEAR COMMITTED DOSE EQUIVALENT FROM ACUTE RADIONUCLIDE EXPOSURES
1

8

10

41

APPENDIX III - ESTIMATES OF MAXIMUM ANNUAL DOSE EQUIVALENT FROM ACUTE RADIONUCLIDE EXPOSURES

72 


\section{ABSTRACT}

This report presents internal radiation dose conversion factors for radionuclides of interest in environmental assessments of nuclear fuel cycles. This volume provides an updated summary of estimates of comitted dose equivalent for radionuclides considered in three previous Oak Ridge National Laboratory (ORNL) reports.

Intakes by inhalation and ingestion are considered. The International Comission on Radiological Protection (ICRP) Task Group Lung Model has been used to simulate the deposition and retention of particulate matter in the respiratory tract. Results corresponding to activity median aerodynamic diameters (AMAD) of $0.3,1.0$, and $5.0 \mu \mathrm{m}$ are given. The gastrointestinal (GI) tract has been represented by a four-segment catenary model with exponential transfer of radioactivity from one segment to the next. Retention of radionuclides in systemic organs is characterized by linear combinations of decaying exponential functions, reconmancied in ICRP Publication 30. The first-year annual dose rate, maximum annual dose rate, and fifty-year dose comitment per microcurie intake of each radionuclide is given for selected target organs and the effective dose equivalent. These estimates include contributions from specified source organs plus the systemic activity residing in the rest of the body; cross irradiation due to penetrating radiations has been incorporated into these estimates. 


\section{INTRODUCTION}

This volume presents tabulations of dose conversion factors for target organs of man due to the inhalation or ingestion of any of the radionuclides listed in Table 1. The present tabulation provides a revised summary of 50-year committed dose equivalents published in three previous reports (Killough, et al.. 1978a; Dunning, et al., 1979, 1981). Annual dose equivalent rates for the first year after exposure and the year in which the maximum effective dose equivalent is received are al so tabulated. Metabolic models, assumptions, and methods recommended by the ICRP (1979, $1980,1981)$ have been used throughout this analysis.

Estimates of dose equivalent presented in this report have been computed using a computer code adapted from the INREM II ( $\mathrm{K}$ illough, Dunning, and Pleasant, 1978b) and RADRISK (Dunning, Leggett and Yalcintas, 1980) computer codes.

Intake of a radionucilde by ingestion and inhalation is considered. In the latter case a particulate form is assumed, with the exception of isotopes of noble gases, ${ }^{3} \mathrm{H}$, and ${ }^{14} \mathrm{C}$. Dynamic models are applied to the estimation of activity and cumulated activity of the radionuclide (and of radioactive daughters that are formed in the body) in the respiratory and GI traces and in other organs which may tend to concentrate the material. These activity integrals for the source organs are multiplied by dosimetric "S-factors" (rem/ $\mu \mathrm{Cl}$-day; Dunning, Pleasant, and K1llough, 1980; Snyder et al., 1974) to provide estimates of dose equivalent to each of a standard ifst of target organs. The terms "source" and "target" refer to the 
rable 1. Radionuclides for which dose estimates are tabulateda

\begin{tabular}{|c|c|c|c|c|c|c|}
\hline Hucilde & $\begin{array}{l}\text { Radloactlve } \\
\text { half-life } \\
\text { (days) }\end{array}$ & $\begin{array}{l}\text { Daughter } \\
\text { nuclide }\end{array}$ & $\begin{array}{l}\text { Branching } \\
\text { fraction }\end{array}$ & $\begin{array}{l}\text { Daugheer } \\
\text { nucilide }\end{array}$ & $\begin{array}{l}\text { Branching } \\
\text { fraction }\end{array}$ & $\begin{array}{l}\text { Spontanezis } \\
\text { fisston } \\
\text { fraction }\end{array}$ \\
\hline 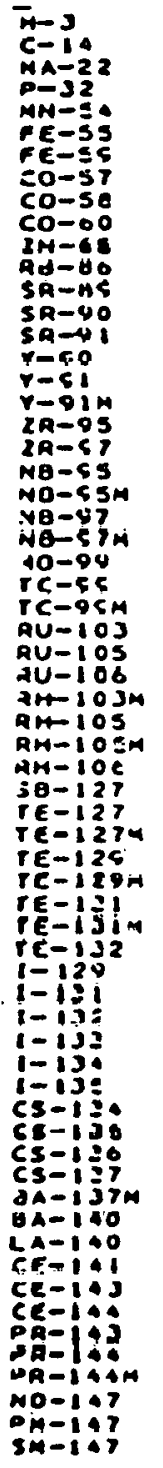 & 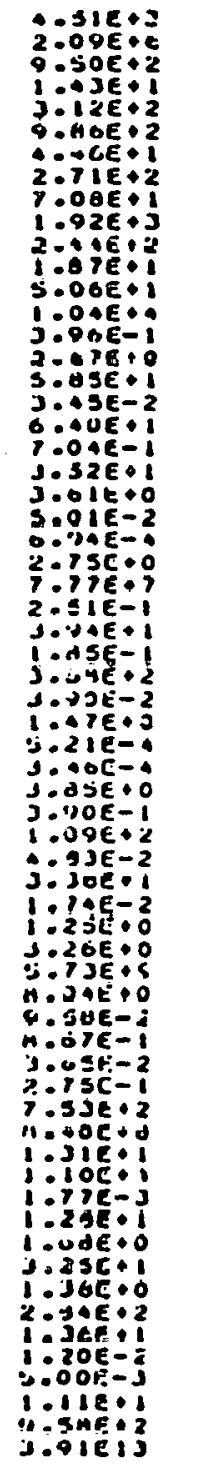 & 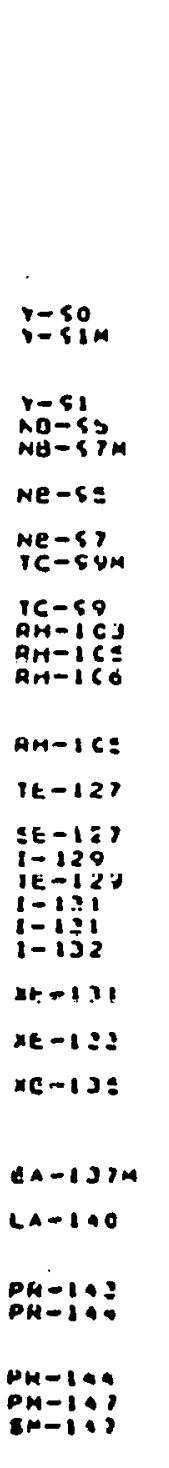 & 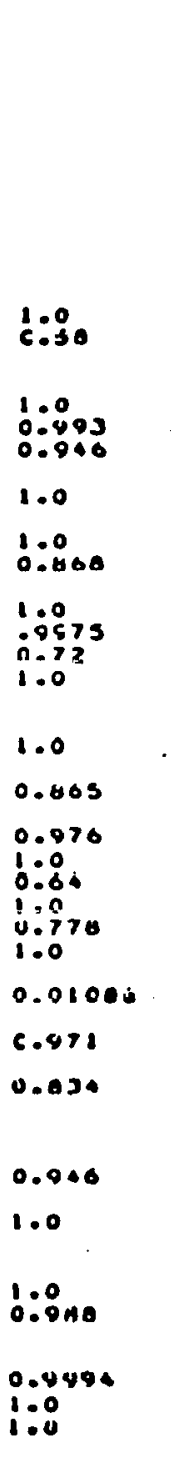 & $\begin{array}{l}\text { rC-99 } \\
\text { op- } 105 M\end{array}$ & $\begin{array}{c}1 \\
0.42 \\
0.007 \\
0.054\end{array}$ & . \\
\hline
\end{tabular}

aphysical data from O. C. Kocher. Muolear Deoay Data for Radionuallded Doourring in

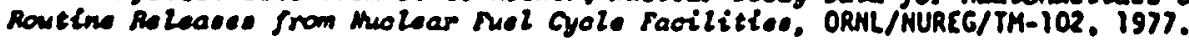


Table 1. (conelnued)

\begin{tabular}{|c|c|c|c|c|c|c|}
\hline Hucllide & $\begin{array}{c}\text { Radloactive } \\
\text { half-ilfe } \\
\text { (days) }\end{array}$ & $\begin{array}{l}\text { Dughter } \\
\text { nuelide }\end{array}$ & $\begin{array}{l}\text { oranchlng } \\
\text { fraction }\end{array}$ & $\begin{array}{l}\text { Dugher } \\
\text { nuclide }\end{array}$ & $\begin{array}{l}\text { Branching } \\
\text { fraction }\end{array}$ & $\begin{array}{l}\text { Spontancous } \\
\text { fisston } \\
\text { fraction }\end{array}$ \\
\hline 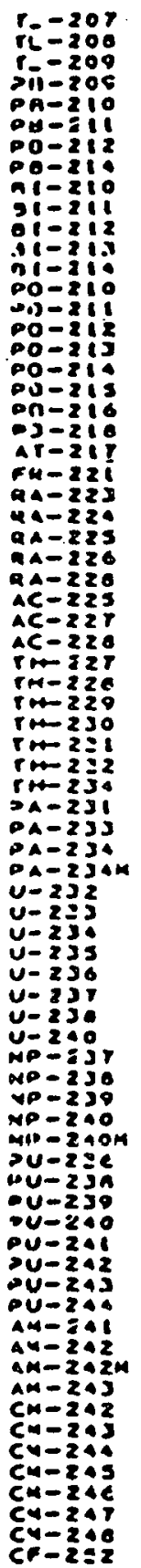 & 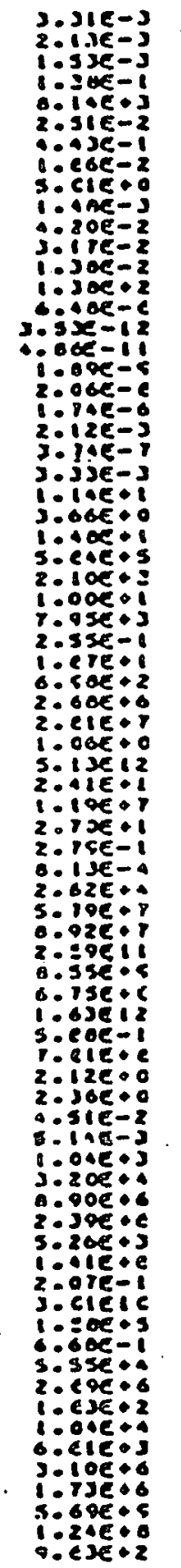 & 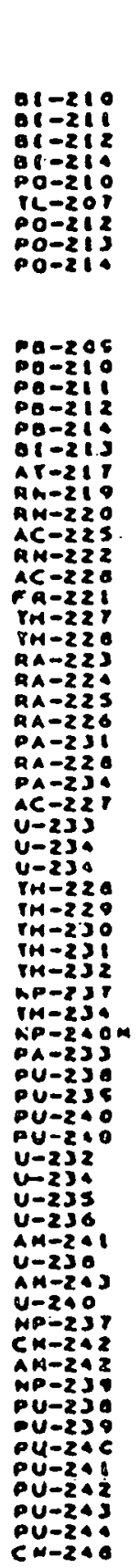 & 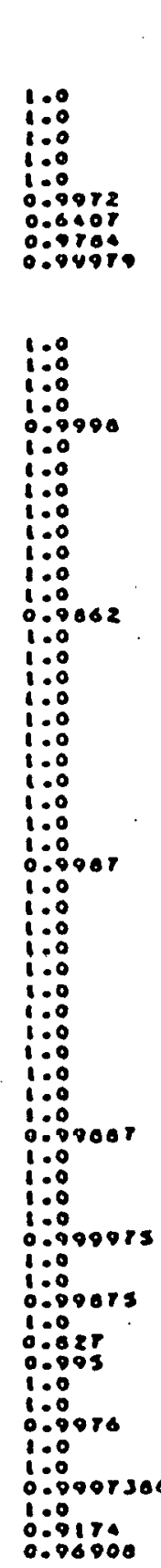 & 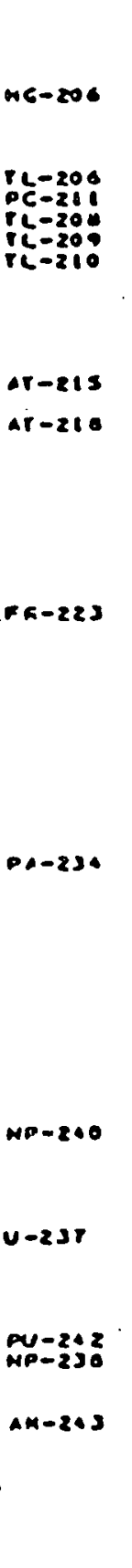 & $\begin{array}{l}2.00-00 \\
1.32-06 \\
0.0020 \\
0.3593 \\
0.0216 \\
0.00028 \\
2.02-06 \\
0.0008\end{array}$ & 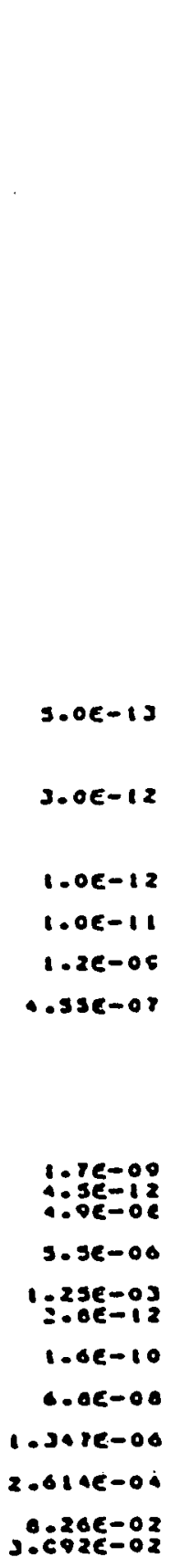 \\
\hline
\end{tabular}


irradiation or organs by emissions from radionuclide activity in other organs.

The dynamic models include the lung model for Inhaled particulates proposed by a task group of the ICRP (Morrow et al., 1966). This model, with parameters from ICRP Publication 19 (1972), is employed to estimate deposition and retention in the major regions of the respiratory tract and to calculatc translocation of the inspired material into the blood and other organs.

The GI tract is represented by a catenary model of four segments (Bernard, 1968) with mean transit times suggested by Eve (1966). The model permits absorption of activity into the blood from any combination of the segments, but $1 t$ is assumed that all absorption 15 from the small intcstine in this report.

Retention of activity in organs other than the respiratory and GI tracts is modeled by linear combinations of up to five exponential functions. Metabolic models reconmended by the ICRP $(1979,1980,1981)$ have been used throughout this report. Specific activity models have been used to compute doses from $\mathrm{H}-3$ and $\mathrm{C}-14$ (Kfllough, 1982; Kfllough, et al.; 1978a).

An important difference between estimates of dose equivalent presented here and those in the previous ORNL reports is the adoption of the ICRP assumptions concerning metabolic behavior of daughter radionuclides produced in the body. In this report, it is assumed that most radioactive 
daughters produced in the body by radioactive decay of a parent isotope adhere to the metabolic models and parameters appropriate for the parent, and that daughter activity produced in an organ compartment remains in that compartment without redistribution. Exceptions are made for radioisotopes of iodine occurring as daughters of tellurium and for noble gases. In the earlier reports, (K1llough, et al. 1978a; Dunning et al., 1979, 1981) each daughter was permitted to assume metabolic characteristics appropriate for that element. These assumptions can impact estimates of dose significantly; for example, the ICRP assumption yields an estimate of the dose to red marrow from ingestion or inhalation of $\mathrm{Pb}-210$, approximately one order of magnitude greater than the estimate produced using the assumption of independent daughter metabolic behavior. Table 2 is provided as a guide to the user to indicate those cases which are most significantly impacted by these metabolic assumptions. The ratio of the 50-year effective comitted dose equivalent (ICRP, 1977) computed using the ICRP metabolic assumptions to that computed using the assumption of independent daughter metabolism is presented for each radionuclide and exposure case where this ratio exceeds the value 2. At present, it is not clear which assumption is more correct, and work is continuing on this problem. The methods used in this report have been chosen for consistency with current ICRP recommendations.

Appendix I gives summary tabulations of 50-year committed dose equivalent (rem/ $\mathrm{HCi}$ ) to selected target organs of a reference aduit. The inhalation tables correspond to particle activity median aerodynamic diameters (AMAD) of $0.3,1.0$, and $5.0 \mathrm{~m}$. The respiratory clearance class of the parent nuclide $(D, W$, or $Y$, where applicable) is shown in the column 
Table 2. Comparison of 50-year effective committed dose equivalents ( $H_{0}$ ) computed using alternate metabolic assumptions discussed in the text

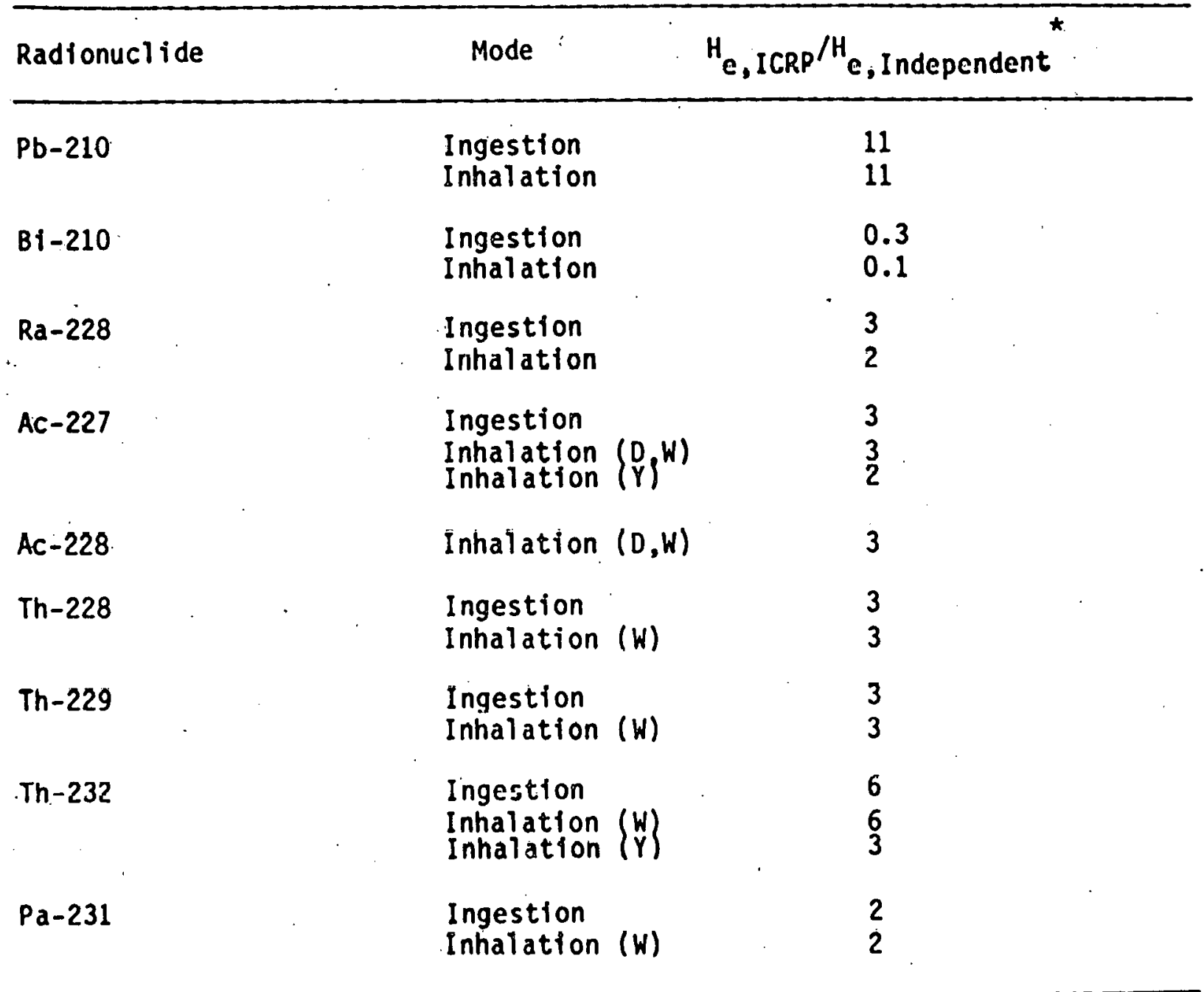

* Ratio of the 50-year effective comitted dose equivalent computed using the ICRP assumption that daughter radionuclides produced in the body retain the metabolic characteristics of the parent radionucilde to that computed using the assumption of independent daughter metabolism. 
labeled "SOL", and the GI tract absorption fraction "FI" is listed. The ingestion table shows only the GI absorption fraction of the parent nuclide, in addition to the dose conversion factors. Inhalation and ingestion cases are presented for each chemical form of a radionuclide considered appropriate (ICRP, 1979, 1980, 1981).

Appendix II presents tabulations of the first-year annual dose equivalent rate (rem/year per $\mathrm{HCl}$ intake) to each of the specified target organs from inhalation and ingestion of each radionuclide.

Estimates of the annual dose equivalent rate to each target organ are presented in Appendix III for the year in which the greatest effective dose equivalent rate (ICRP, 1977) is received, if different from the first year. For radionuclides with short radiological half-lives or rapid biological turnover in the body, the maximum effective annual dose rate occurs during the first year. For long-lived radionuclides, the maximum value of ten occurs in the second year. In situations where the dose is dominated by slow ingrowth of a daughter radionuclide, the maximum effective annual dose may occur many years after intake.

All dose equivalents presented in this report for alpha-emitting radionuclides utilize a quality factor for $Q_{\alpha}=20$ as recommended by the ICRP (1977). 
REFERENCES

Bernard, S.R., 1968, "A Model for Estimation of Mean Activity Present in Segments of the Gastrointestinal Tract at Any Time Post Ingestion " Heal th Physics Division Annual Progress Report for Period Ending July 31, 1968, ORNL-4316, pp. 283-287.

Dunning, D.E., S.R. Bernard, P.J. Wal sh, G.G. Killough, and J.C. Pleasant, 1979, Estimates of Internal Dose Equivalent to 22 Target Organs for Radionuclides Occurring in Routine Releases from Nuclear Fuel Cycle Facilities, Vol. II, ORNL/NUREG/TM-190/V2.

Dunning, D.E. J.C. Pleasant, and G.G. Killough, 1980, SFACTOR: A Computer Code for Calculating Dose Equivalent to a Target Organ per Microcurte-Day Residence of a Radionuclide in a Source Organ Supplementary Report, ORNL/NUREG/TM-85/S1.

Eve, I.S., 1966, "A Review of the Physfology of the Gastrointestinal Tract in Relation to Radiation Doses from Radioactive Materials," Health Physics, 12: 131 .

International Commission on Radiological Protection (ICRP), 1972b, The Metabolism of Compounds of Plutonium and Other Actinides, Pubiication 19, Pergamon Press.

International Comrnission on Radiological Protection (ICRP), 1977, Annals of the ICRP 1, 1, Publication 26, Pergamon Press.

International Commission on Radioiogical Protection (ICRP), 1979, Limits for Intakes of Radionuclides by Workers, Publication 30, Part 1, Annals of the ICRP, Vol. $3(3 / 4)$, Pergamon Press.

International Cormission on Radiological Protection, 1980, "Limits for Intakes of Radionucl Ides by Workers."ICRP Publication 30, Part 2. Annals of ICRP. Vol. $\$(3 / 4)$, Pergamion Press.

International Commission on Radiological Protection, 1981, "Limits for Intakes of Radionuclides by Workers." ICRP Publication 30, Part 3 , Annals of the ICRP, Vol. $6(2 / 3)$, Pergamon Press.

Killough, G.G., 1982, "Derivation of Dose Conversfon Factors for Tritium", NUREG/CR-2523, ORNL-5853.

Killough, G.G., D.E. Dunning, S.R. Bernard, and J.C. Pleasant, 1978a, Estimates of Internal Dose Equivalent to 22 Target Organs for Radionuclides Occurring in Routine Releases from Nuclear Fuel-Cycle Factlities, Vol. I, ORNL/NUREG/TM-190. 
9

Killough, G.G., D.E. Dunning, and J.C. Pleasant, 1978b, INREM II: A Computer Implementation of Recent Models for Estimating the Dose Equivalent to Organs of Man from an Inhaled or Ingested Radionuclide, NUREG/CR-0114, ORNL/NUREG/TM-84.

Kocher, D.C., 1977, Nuclear Decay Data for Radionuclide Occurring in Routine Releases from Nuclear Fuel Cycle Facilities, ORNL/NUREG/TM-102.

Morrow, P.W., D.V. Bates, B.R. Fish, T.F. Hatch, and T:T. Mercer, 1966 , "Deposition and Retention Models for Internal Dosimetry of the Human Respiratory Tract," Health Physics, 12: 173.

Snyder, W.S., M.R. Ford, G.G. Warner, and S.B. Watson, 1974, A Tabulation of Dose Equivalent per Microcurfe-Day for Source and Target Organs of an Adult for Various Radionuclides, Part 1, ORNL-5000. 
APPENDIX I

ESTIMATES OF 50-YEAR COMMITTED DOSE EQUIVALENT FROM ACUTE RADIONUCLIDE EXPOSURES 
(REM MICROCURIE)

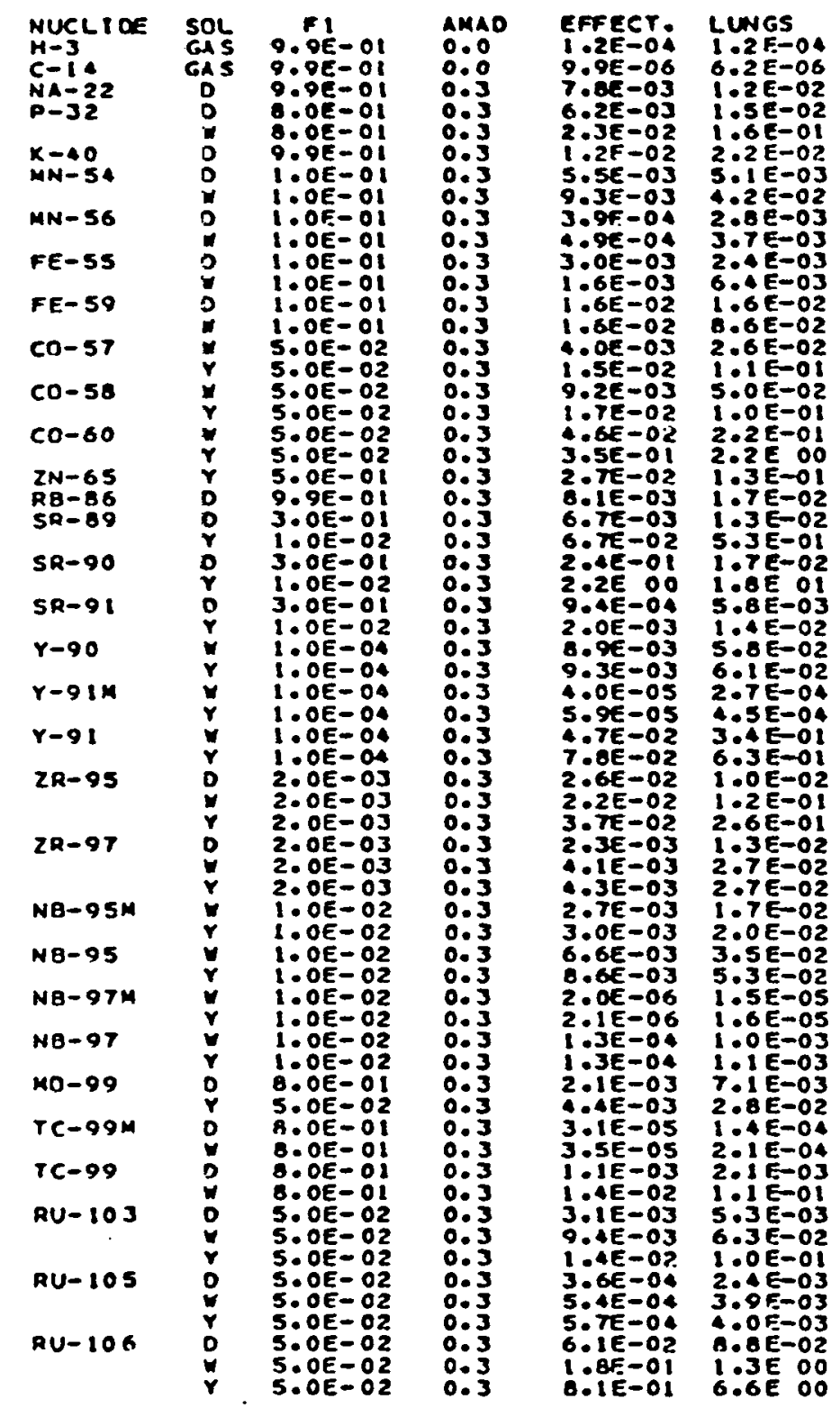

S WALL SI WALL $7: 3 E=06 \quad 1: 3 E-04$ $1.0 \mathrm{C}-03$ 9.4E-OA

$\begin{array}{ll}1.4 E-03 & 9: 4 E-04 \\ 6.3 E-03 & 5.7 E-03\end{array}$

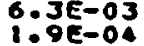

$1.7 E-04$

\begin{tabular}{l}
$1.3 E-03$ \\
\hdashline $2 E=04$
\end{tabular}

1:A $1: 0=02$

$7: 3 E-03$
$1: 1 E-03$

3.: $3 E-03$

$0 E-03$
2.85

$2: 8 E-02$

$1: B E=0$

$2: 7 E-03$
$1: 0 E-03$

$8.8 E=0.4$

2: $1 E=03$

2.8E-04

$9: 2 \in=04$

B:0E-06

$9: 0 E-06$

$9.3 E=04$

$4.7 E-03$
$3: B E-03$

$1.1 E-02$
$5.5 E E-04$

$1: 0 E=03$

5. $6 E-04$

6.5E-OT

$8.1 E-07$
$3.6 E-05$
$4.6 E-05$

$\because .8 E-05$
$\because .8 E-04$

6.: 4 E-O4

$7.1 E-05$

$3.9 \mathrm{E}-0.3$
$9.0 \mathrm{E}-0.3$

$2.3 E-03$
$2.1 E-03$

$2.1 E-03$

$1.7 E=04$

$2.0 E-04$
$3.2 E-02$

I. 1 IE-02
5. TE-0.
$3: 1 E-03$
$2: 9 E-03$

I. $\mathrm{SE}-\mathrm{OA}$

(1)

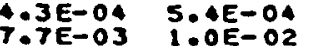

$5.0 E-03$

$6.5 E-04$

$3: 3 E-03$

$1.2 E-03$
$1.2 E-03$
$3.9 E-03$

$\begin{array}{ll}2: 4 E-03 & 3.9 E-03 \\ 2.7 E-03 & 4.2 E-03\end{array}$

$\begin{array}{ll}1.3 E-02 & 2: 0 E-02 \\ 3.5 E-02 & 5.0 E-02\end{array}$

$\begin{array}{ll}5.6 E-03 & 7.5 E-03 \\ 2.4 E-03 & 2.5 E-03\end{array}$

$1.1 E-03$ 2:1E-03

$\begin{array}{ll}2.2 E-03 & 1.3 E-02 \\ 409 E-03 & 5.5 E-03\end{array}$

$\because 2 E-03 \quad 2 . J E-02$

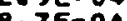

$2: 4 E-03$

$\begin{array}{ll}2.2 E=03 & 1: 1 E-02 \\ 2.5 E-03 & 1: 3 E=02\end{array}$

$4.2 E-06$ 1. IE-05

$2.6 E-03 \quad 1.4 E-02$

$2.3 E=03$
$3.0 E=03$

3. $3 E-03$

$6.0 E-03$

$6.3 E-03$

60

$1.9 E-03$
$2.4 E-03$

$6.0 \mathrm{E}-04$

$2.5 E-03$

$\begin{aligned} & 1.8 E-03 \\ & 3\end{aligned}, 1 E-03$

$1: 4 E-07$
$A: G E=07$

2.0E-OT

$3: 7 E-07$
$1.80-05$

$2.4 E-05$
$5.4 E-04$

5:- $:=03$

i.ie-05

6:7E-06

1.SE-OA

$6.6 \mathrm{E}-04$
$2.3 \mathrm{E}=03$
$3.9 \mathrm{E}-03$

$\begin{array}{ll}1: 5 E-03 & 3.9 E-03 \\ 1: A E-03 & 3.7 E-03\end{array}$

1.6E-04

3.0E-OA

$5.0 E-04$
$6: 4 E-04$

$\begin{array}{ll}2.0 E-04 & 6.4 E-04 \\ 3: 1 E-02 & 3: 5 E-02 \\ 15 E-02 & 4.6 E-02\end{array}$

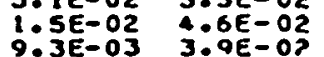

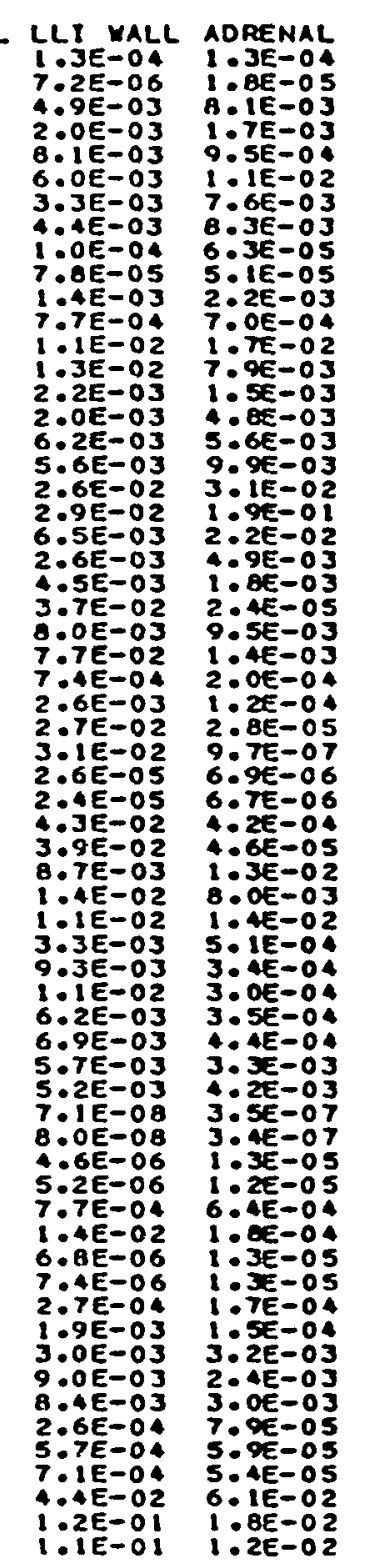

BL YALL

$2.2 E-04$ $6.3 E-03$

1: $\mathrm{TEE}=03$

$1.1 E-02$

1. $4 E-03$

3. $3 E-05$

2. $2 E-03$

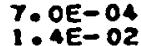

3. $B E-03$

$4.2 E=04$
$2.1 E-04$

$1: 2 E=03$

$1.3 E-02$

$6.5 E-03$

$\therefore B E=03$

$2.3 E-05$

P: $5 E=03$

$1 . .4 E-04$

2. $\theta E-05$

$9: 7 E=07$

$40 E=07$
$4.0 E-0.04$

2.:2E-05

: 6 E 003

$1.5 E-03$

3. $B E-04$

1: $3 E=-04$

$8: 1 E=05$
$: 0 E-05$

$3.9 E=00$

$1.0 E-08$

2.3 $3 E=06$

$4.5 E-04$

$\because 9 E=06$

$2.2 E=06$
$1.7 E=0.04$

3. $5 E-04$

$8.2 E=04$

S. $1 E-05$

i: TE-05

$6: 1 E-02$
$1.6 E-02$

$1.6 E-02$
$: 6 E-03$ $x$ IONEYS LIYER OREAST

$7: 0 E-03$ 6:jE-0J

$.7 E-03$ 1.TE-03 $1.7 E-03$

9.5E-04 0.SE-04 9.5E-04

$1 \cdot 1 E-02 \quad 1.1 E-02 \quad 1 \cdot 1 E-02$

I.9E-02

$5.1 E-05$ I:IE-04

3.JE-05 $7.1 E-05$

T. $0 E-0.0$

1:TE-02

Y. $1 E-03$

$7: 11 E-03$

$2.20-02$

$1: 1 E-02$
$1: 0 E-003$
6

$4: 4 E=03$

$\because A E-03 \quad: 0 E-02$

$2 \cdot 2 E-02 \quad 4.3 E-02$

:-3E-02 2:1E-02

$\begin{array}{ll}1: 0 E-03 & 1: 9 E-03 \\ 9 & 0 E-03\end{array}$

作

$0.5 E-03$
$1.4 E-03$

$1.7 E-04$ 1.SE-O

$2.0 E-05 \quad 7.3 E-04$

$\begin{array}{ll}2.7 E-07 & 2.5 E-05 \\ 3.4 E-06 & 1.8 E E-05\end{array}$

$3.1 E-06 \quad 7: 1 E-06$

$3.3 E-05$ 9.6E-0

$8.00-035.8 E-03$

$5.9 \varepsilon-03 \quad 1.3 \varepsilon-02$

2.1E-04 3.5E-04

$2.2 E-03$
$1.0 E-03$

1.6E-07 $3.5 E=07$

$6: 3 E-06$

$\begin{array}{ll}6.0 E-03 & 6.4 E-03 \\ 2.6 E-04 & 3.5 E-04\end{array}$

5. $8 E-06$ 1.2E-05

1.7E-04 2.2E-O4

1.SE-04 $1: 9 E-04$

$1 . A E-03 \quad 2.3 E-03$

$6.3 E-05$

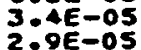

$6: 1 E-02$

$1: 7 E-02$
$7.6 E-03$

5. $9 \mathrm{EE}-05$

$6.1 E-02$

i. $1: \begin{aligned} & 7 \\ & 1.02\end{aligned}$

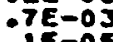

5.1E-05

$3.2 E-03$
$0 E-04$

$1: 5 E-02$
$5.0 E-03$

(1)

.

5.:TE

$2.22 E-02$

SE-O2

$40.2=03$

2.AE-05

104

T.9E-05

9 :7E-0

$4.2 E-06$

3.7E-06

.6E-05

:.3E-03

6E-OJ

2. $A E=04$

$2 \cdot 0 E=04$

$2.6 E-04$

2

$2.1 E-07$

$1.9 \varepsilon-07$

$7.7 E-06$

. $B E-04$

$1.2 E-04$

$7.2 E-06$

l.7E 1.04

$2.9 E-03$

$1.6 E=03$
$1.8 E=03$

उ. $B E=05$

$6.1 E-02$
$1.7 \varepsilon=02$
X.JE-04 1.2E-04 1.2E-0.

$1.9 E-04$ 3.2E 04

2:1E-04 $3: 4 E-04$

$5.6 E-06 \quad 1.2 E-05$

$1.7 E-02$
$8.5 E-03$ 
INHALATION DOSES AT 50 YEARS

(FEMUMICROCURIE)

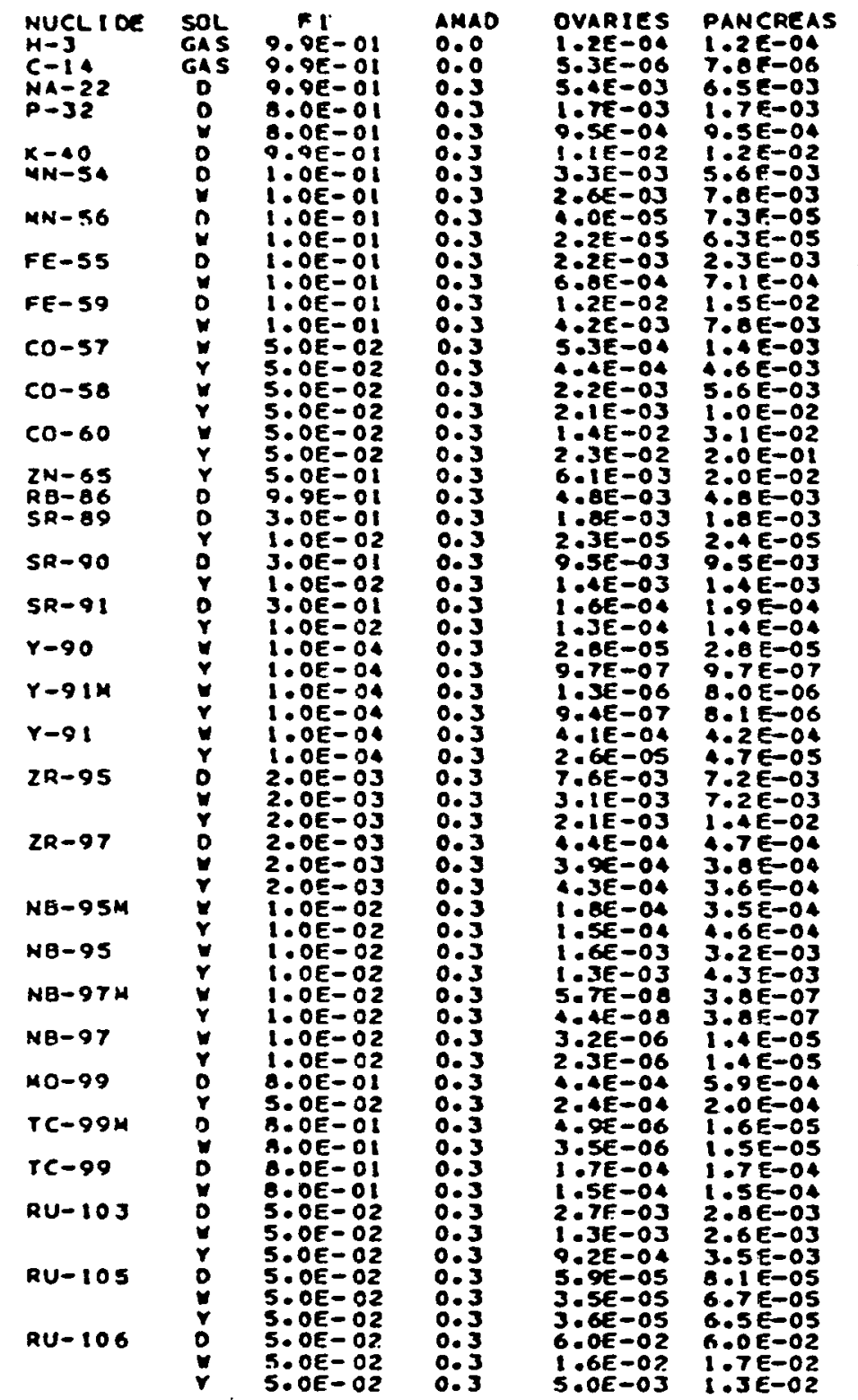

BRAIN

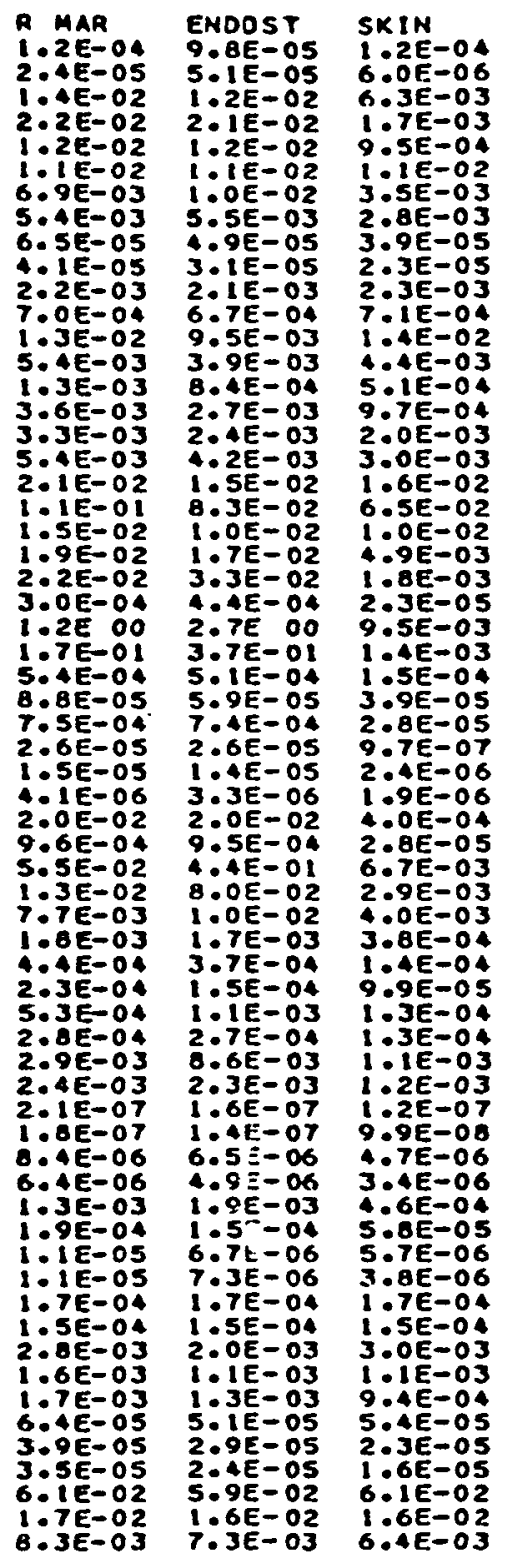

- 2E-0 TESTES 6. BE-06 5.4E-06 $1.7 E-03$ S.TE-OJ

E-04 $9.5 E=0$

$\begin{array}{ll}1 \cdot 1 E-02 & 1.1 E-02 \\ 3.8 E-03 & 2.5 E-03\end{array}$

$\begin{array}{ll}6 \cdot 7 E-03 & 9 \cdot 2 E-04 \\ 6.6 E-05 & 2 \cdot B E-05\end{array}$

$5.7 E-05$ B.TE-06

$1.2 E-02$ 2.2E-03

$3.7 E-03 \quad 6.9 E=04$

1. IE-02 3. DE-03

1.2E 3.1E-04

$5.0 E-03 \quad 6.7 E-0.4$

$20 E=03$

1.

$1 . T E-01 \quad 8.6 E-03$

$1.9 E-03$
$1.0 E-02$

$1.0 E-03 \quad 1.8 E-03$

i: $9 \mathrm{EE}-03$

$2.4 E-05$

$1.4 E-0.3$

2.JE-05

(1)

$1.2 E-a .4$

i.AE-03

$1.2 E=05$

$2.8 E-05$
$9: 7 E-07$

1. $1 E-06$

a

$6: 3 E-06$

$411-04$

2. $3 E-05$

$4.4 E-a 5$

$6.4 E-03$

$1.25=02$

$3: 0 E-03$

$1.4 E-03$

$3: \begin{array}{ll}E-a \\ 3\end{array}$

3.0

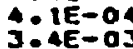

$3.0 E-a 3$

$3: 2 E-a 7$

$1.2 E-05$
$2.3 E=05$

$9.7 E-07$

$6.2 E-07$
$111 E-07$

$2.0 E-05$

$4.9 E-03$

1. $1 E=03$

2. $A E=04$

$3.8 E-05$
$1.9 \varepsilon-04$

$4.3 E-05$

2. $9 E-05$

5.:2E-04

-1
2.5

1. $1 E-a 7$

$2.0 E-04$
$2.1 E-08$

$1.1 E-06$

$4.5 E-04$
$2.2 E-05$

$5: 0$ -

$1 \cdot 3 E-06$
$2 \cdot 5 E-07$

$1: T E-a 4$

1: $2 E E=05$

3.: 1 - OS

$4.4 E=06$

$7 E-04$ I:TE-OA

$3: 0 E-03$

$1: 5 E-04$
$2: 7 E-034$

$\begin{array}{ll}2: 2 E-03 & 5.7 E-04 \\ 2.7 E-03 & 1.9 E-0.04\end{array}$

$7.5 E-05$ 4. $4 E-05$

$\begin{array}{ll}5.6 E-05 & 1.0 E-05 \\ 5.2 E-05 & 3.6 E-06\end{array}$

$6: 1 E-02$

$6.0 E-02$

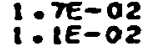

THYMUS THYROTD UTERUS

$\begin{array}{lll}1.1 E-04 & 1.2 E-04 & 1.2 E-0 \\ 7.1 E-06 & 0.5 E-06 & 7.0 E-06\end{array}$ $5.8 E-03 \quad 6.9 E-03 \quad 5.2 E-03$ $0.5 E-04$ OSE-0 $1.1 E-02$ 1. IE-02 $1: 1 E=02$ I.TE-O3 1.TE-03 $9.1 E-05$ औ.9E-05 3.6E-05 9.5E-OS $3.4 E-05$ 1.6E-OS $7.1 E-04 \quad 6.9 E-04$ 1.1E-02 $5.9 E-03$ 3.6E-03 $2.0 E-03$ 7.AE-O4 $4.0 E-04$ 7.7E-03 $1.7 E-03 \quad 3.0 E-04$ $1.6 E-02$ 5.5E-03 1.1E-03 $1.2 E-02$
$2.4 E-02$ $3.2 E-02 \quad 1.6 E-02 \quad 6.0 E-03$ $1.0 E-03 \quad 1.9 E-03 \quad 4.0 E-03$ $2.4 E-05 \quad 2.4 E-05 \quad 2: 3 E-05$ $1,4 E-03$ i. $2.3 E-04$ I:TE-OA 1:AE-O4 $1.8 E-046.1 E-05 \quad 6.8 E-05$ $\begin{array}{lll}2.8 E-05 & 2.8 E-05 & 2.8 E-O S \\ 9.7 E-07 & 9.7 E-07 & 9: 7 E-0 O\end{array}$ 9.9E-06 $3.7 E-07$ $1 \cdot 0 E-05 \quad 3.3 E-05$ $7.1 E-05$ 3:4E-OS

$6.4 E-03$

$2.2 E-02$

\begin{tabular}{l}
$5.2 E=04$ \\
\hdashline$T E=04$
\end{tabular}

$\because \because 4 E-04$

$7.0 E=04$
$406 E-03$
0.07

$6.7 E-03$

$5.5 E-07$
S. $4 E-07$

$1.8 E-05$

$4.8 E-04$
$2.6 E-0.4$

$1: 5 E-0 S$

1.9.

30

$3.2 E-03$

$9.1 E-05$

B.AE 0 -OS

$1.06-05$
$6.0 E-02$ $1.0 E-02$
$1.8 E-02$

$\begin{array}{ll}4.5 E-03 & 1.9 E-03\end{array}$

$4.2 E-043.7 E-0$.

1.TE-O4 2.1E-04

$13 E-04 \quad 2.0 E-04$

$\begin{array}{ll}2.3 E-04 & 6: A E=05 \\ 1.8 E-03 & 0.3 E=0.04\end{array}$

$2.3 E-03$ 6.2E-0.

2:0E-aY 4.6E-08

7. $3 E-06 \quad 2.7 E-06$

$42 E-04$ T. $3 E-04$

$7 \cdot 9 E-05$ i:1E-0.

5:JEE-OS $2: T E=06$

$4 . A E=03$ i.7E-OA

$\begin{array}{ll}3.8 E-03 & 1.5 E-04 \\ 2.9 E=03 \quad 2.5 E-0.03\end{array}$

$1: 4 E-03 \quad 7: 9 E=04$

6: $3 E-\infty \quad 5: 1 E-05$

$\begin{array}{ll}2: 3 E=05 & 2.3 E-05 \\ 2.6 E=05 & 2.1 E=05\end{array}$

$\begin{array}{ll}1: 7 E-02 & 1: 6 E-02 \\ 8.2 E-03 & 0.6 E-03\end{array}$ 
(REM/MICROCURIE)

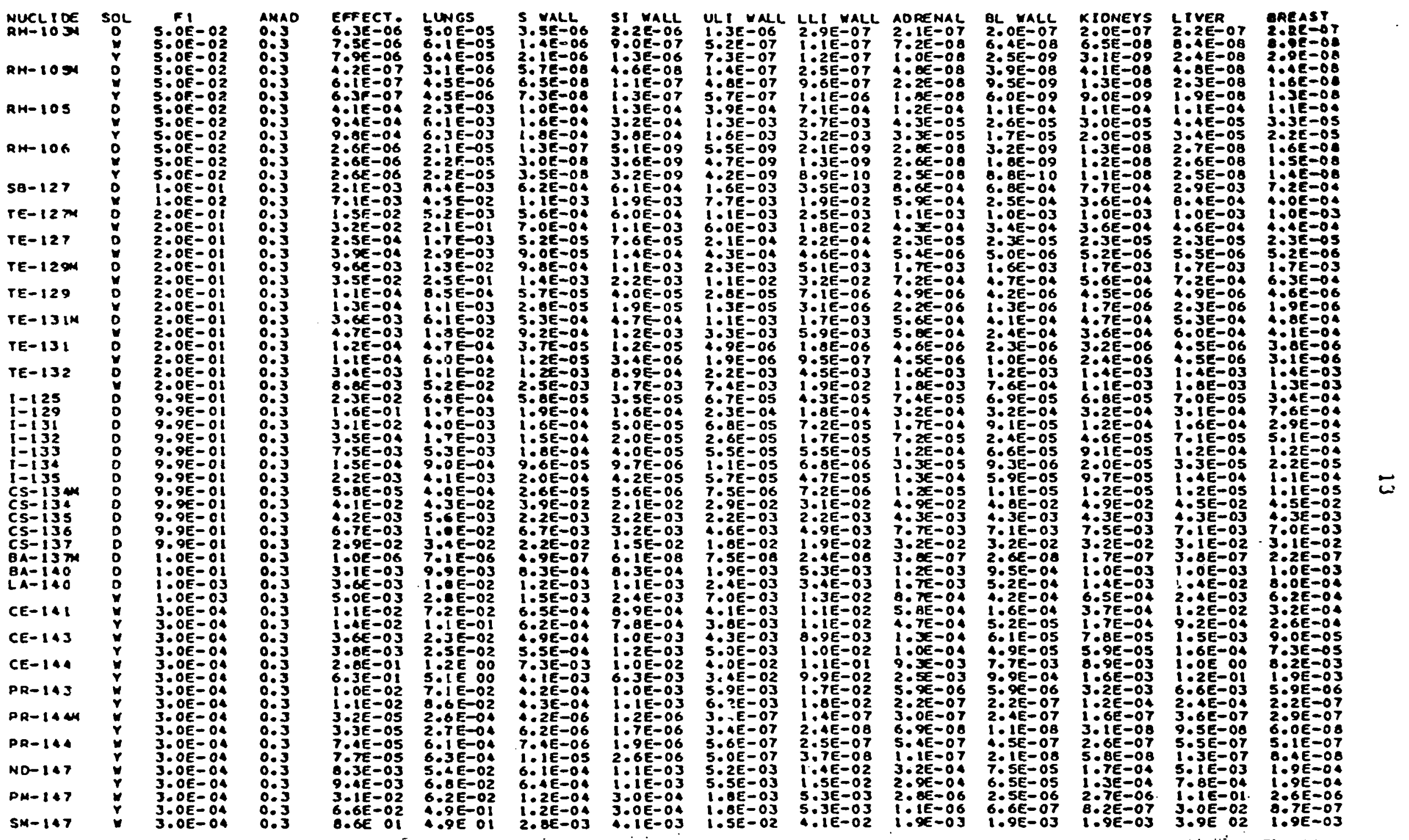


(REM MLPACROCURIE)

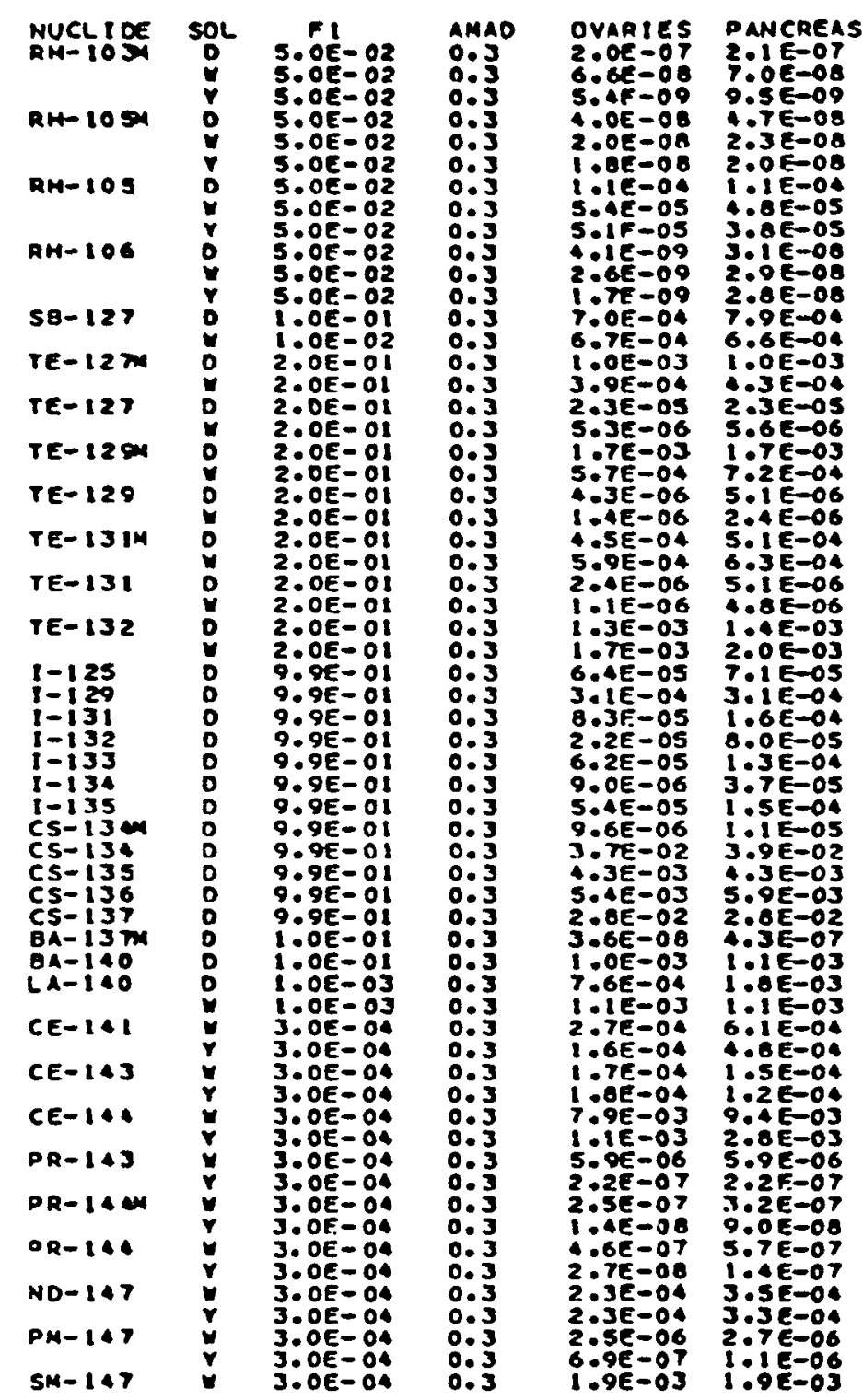

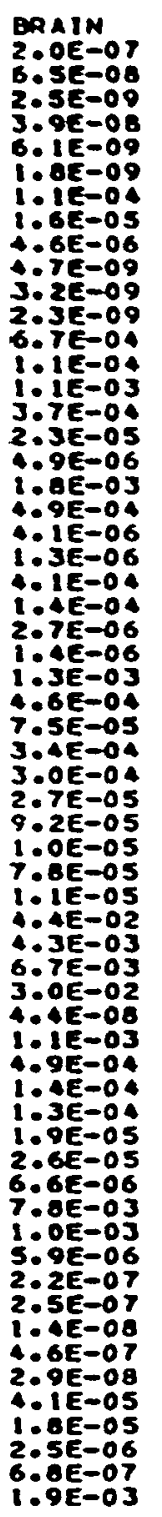

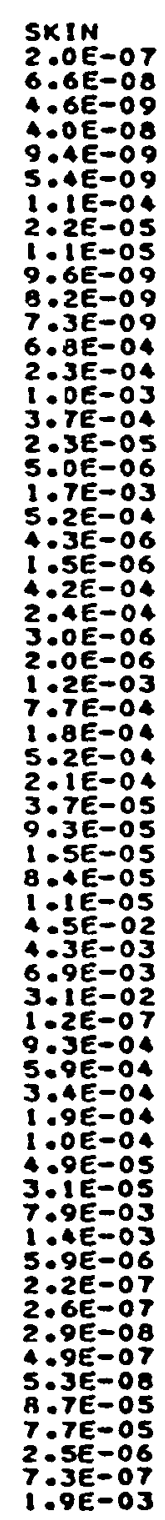
$5: 1 E-0,7$
$3: 0 E-08$ $1: \theta e-08$ 2: 10 : : $1 E=04$ $3: 2 \in=05$ $2.3 \mathrm{~s}=08$ 2.: $5: G E-09$ $1: 05=03$

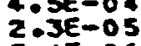
S. $6 \in$ i:secos S.

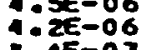
$1:-4=0.03$ 6 $3.06-0$.

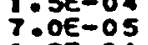
$1: 2 E-04$
$3: 3 E-05$ $1: 3 E=04$ 5: $5 E=05$ $4.5 E-02$ $4.3 E-03$

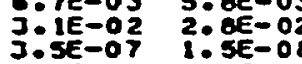
OE-OJ $A . A E-O A$ TAE-AJ $1.2 E-04$ $\because 2 E-03 \quad 3.1 E-05$ $\begin{array}{lll}A E-04 & 1: A E=05 \\ 0.4 E-01 & 7: 7 E-03\end{array}$ $300 E-01$ 9:AE-OOA $\begin{array}{ll}5.9 E=06 & 5.9 E-06 \\ 2.2 E-07 & 2.2 E-07\end{array}$

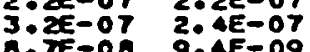
B.TE-0. $9: A E-09$ 1. BE-O? $1: 0 E-08$ 2.6E-04 $1: 0 E-05$ $1.0 E=06 \quad 6.6 E=07$ $1: 9 E=03 \quad 1: 9 E=03$
TMYMUS 2.1E-07 1 . $3 E-08$ 3. $0 E-08$ $2.7 E-00$ $1,1 E-04$
$5.5 E-05$ $4.5 E-05$ $\because 2 E-08$ $4.0 E-08$ $7: 6 E=04$ $0.3 E-0$ a:OE-OA 2:3E-05 $5.7 E-06$

$1.6 E-03$ 8.5E=04 S.2E -06
$2 . \theta E=06$
S $6.3 E-04$ 8.9E-04 $7.4 E-06$ $1.5 E-03$ $2.6 E-03$
$2.0 E-04$ $7.8 E-0$ 1:IE-0. $2.2 E=04$ $2 \cdot 3 E-04$ 1:1E-05 $4: 3 E=03$ $2: 0 E-02$ $9.6 \varepsilon=04$ i. $.3 E=03$

$7: 2 E-04$

1. $7 E=04$ $1.5 E=04$ $3.8 E-03$ $2 \cdot 2 E-07$ $3.7 E-07$
$1.9 E-0.7$ 6.SE-07 $2.2 E-07$
$: 2 E-0.04$ $.6 E=04$
$2.6 E=06$ $1: 3 E-06$
THYROID UTERUS 6:5E-06 $2.7 E=09$
$3.5 E=08$
$3.2 E=00$
$1.1 E=08$
$1.1 E=00$ $2: 5 E-05$ I.OE 1.4E-05 $3 \cdot 1 E-D$ $1.5 E-0.3$ 3.5E-OO $1.3 E-08$ 1.2E-09 $7.2 E-046.3 E-04$ $3.2 E-04$
$3.2 E=04$
$1.0 E=03$
$3.0 E-03$ $3.6 E-04$ J.5E-0 5.1 $1 E-06$ 5.1E=0

$1.7 E-03$ 1.6E-03

$5.6 E-04 \quad \therefore B E=04$ i: TE-06 $1.4 E=06$ 1. $6 E-02$ 3.8E-0 $5.6 E-02 \quad 3.1 E=04$ $1: 2 E-03 \quad 2: 0 E-06$ 3.AE-03 B:AE-0 $7.5 E-01$ 6.2E-0S

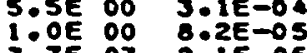
2.1E-0S $2.3 E-01 \quad 6.0 E-05$ 5.6E-02 5.6E-0S

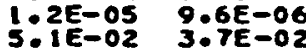
$1.3 E=03 \quad 4.3 E=03$ $8.6 E-03 \quad 5.6 E-03$ $3,3 E-02 \quad 2.8 E-02$ $2.1 E-07 \quad 2.8 E-08$ $5.3 E=04$ 5.BE=0 S.ge-0. $1.6 E-04 \quad 7.5 E=05$ $5.7 E-05 \quad 8.2 E=05$ 7:0E-03 $7: 0 E-03$ 5.9E-06 $5.9 E-06$ 2.2E-07 2.2E=0 3.6E-0B 1.AE-0. $5.0 E=07$ A.0E-07 $7 \cdot 2 E-08 \quad 2.7 E=0$. $1.2 E-04$ 9.1E-0S $7: 7 E=07$
$1.9 E-03$
$1: 9 E=07$ 
INMALAT ION DOSES AT SO VEARS

(REM/MTCROCURIE)

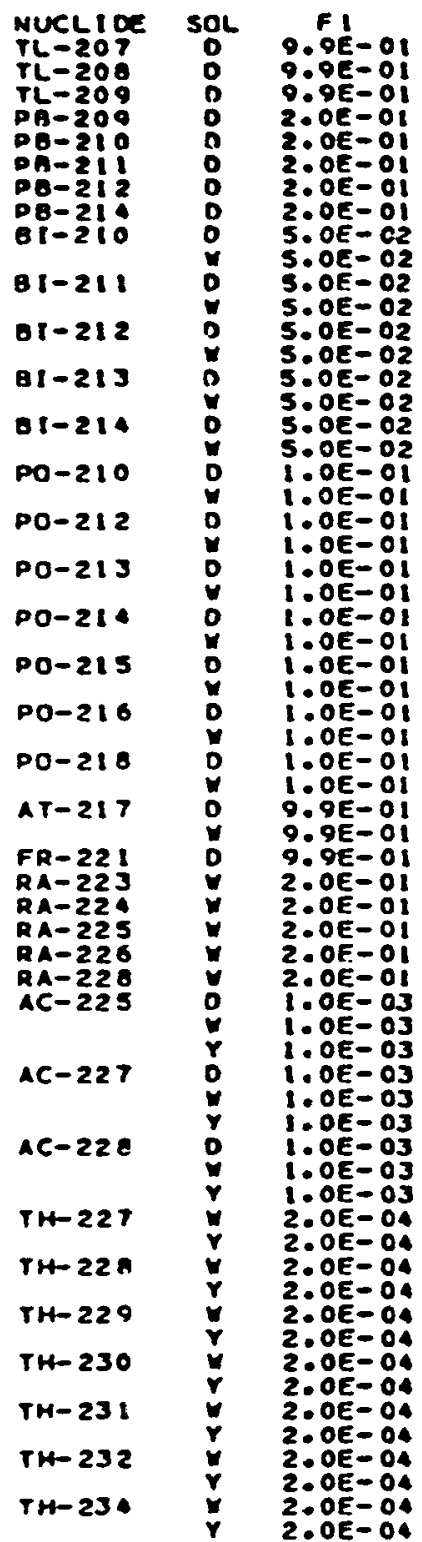

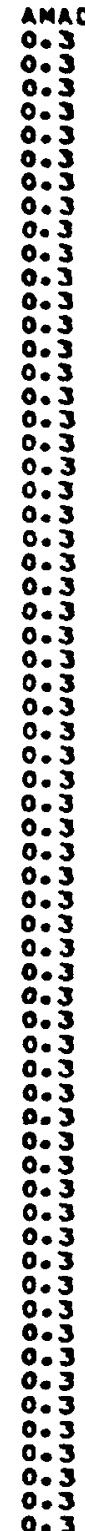

EFFECT: LUNGS

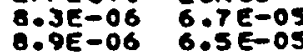
P.TE-O6 S.OEOS i.SE O1 $1: 3 E$ DO 1:2E-02 $1: 0 E-0 !$ $\begin{array}{ll}: 2 E-02 & 0.5 E-02 \\ 0.0 E-02 & 1.5 E-02\end{array}$ $3: j E=012: 7=00$ :.ic उ:OE-02 $2.2 E-01$

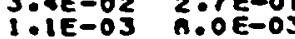
$1: 2 E-03 \quad 9: T E \rightarrow=03$ $1: 1 E-02 \quad 9: 1 E-02$ l:IE : 3.0 E : $3.4 E-12 \quad 2 \cdot 0 E-11$ A.AE-11 $3: 7 E-10$

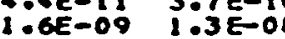
1.6E-09 1:3E-08

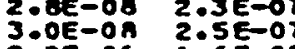
$2.2 E-06 \quad 1.6 E-05$

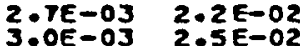
3.0E-OP 2.5E-06 $\begin{array}{ll}3.0 E-07 & 2.5 E-06 \\ 4.6 E-03 & 3.0 E-02\end{array}$ I:JE O1 $1:$ IE O2 S.JE $00 \quad 706$ O OI 1.SE OI $1.0 \mathrm{E}$ O2 B.TE OO THE :J

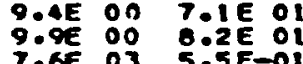

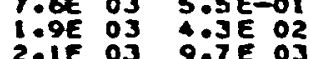
2.1E 03 9.7 E. 03 $\begin{array}{ll}1.1 E-01 & 2.2 E-01 \\ 2.1 E-01 & 1.6 E\end{array}$ $\begin{array}{lll}2.1 E-01 & 1.6 E & 00 \\ 2 . J E & 01 & 1.5 E\end{array}$

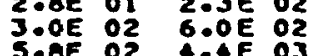
S.AE O2 $4.4 E$ : 03 $\begin{array}{llll}1.0 E & 03 & 3.9 E & 02 \\ 2.2 E & 03 & 9.6 E & 03 \\ 3.0 E & 02 & 1.0 E & 02\end{array}$

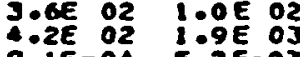
9.1E-04 $5.2 E-03$

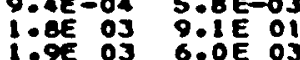

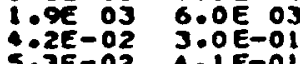

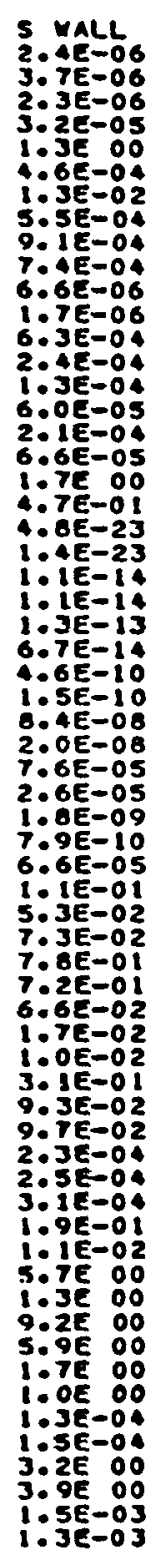

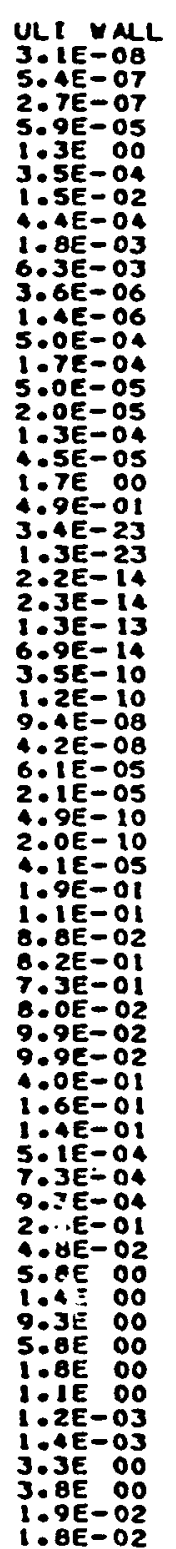

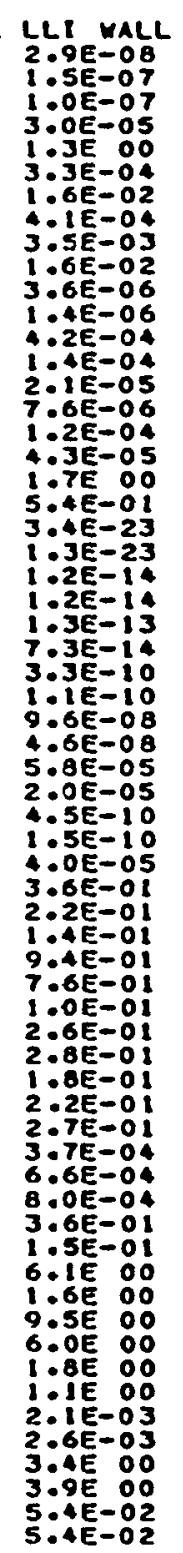

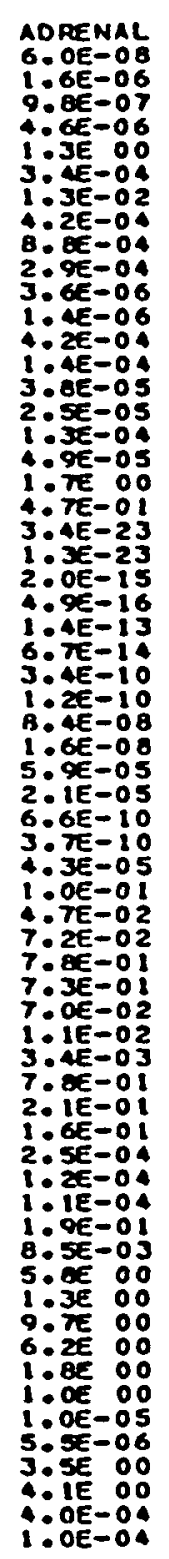

5. YALL 2.0E-07 1.6E-06 I.JE 00 1:JE-O2 $1.1 E=04$
$B .0 E=04$ $2.9 E-04$

$1.4 E-06$

A. $1 E=04$

I: $8 E-05$

1. $2 E-04$

A. JE-OS

$A 7 Z-O$

1.: $3 E-23$

2. $0 E-15$

$1.4 E-13$

3. $3 E-10$

$1.2 E-10$

1. $5 E-08$

2. $0 E-05$

i.: $7 E-10$

$4: 1 E-05$

$\therefore: 7 E-02$

$7: 0 E=02$

7.2E-01

$8: 4 E-02$

$1 \cdot 2 E-03$

3. $6 E-02$

$3.2 E-05$
$3.1 E-05$

3. $1 E-05$

1. $9 E-01$

5. 菠:0 1.JE 00 5. ᄀE 00 $1: 0 E: 0$ 7.26-06 2. IE :D 3. $5 E-04$
KIDNEYS LIVER

1.:E-O7 $5.8 E-08$
$1.2 E-06$
$2.1 E-06$ $1.1 E-06$ 1:1E-06 $2.9 E$ OL $6.3 E$. 1.1E-04 $2,4 E-04$ 2.SE-0. S:3E-04 $2.5 E-01$

8.3E-02

$1.6 \mathrm{E}=05$

$2: 0 E-02$

6.4E-0.

3. $3 E-03$

$5.2 E: 1$

$3.0 E-27$

$1: 6 E-1$.

$4.1 E-12$

5: AE-10

$1.9 E-10$
$1.4 E-06$

$1.1 E-0$

$\because 0 E-05$

$1.9 E-10$

$2.2 E-06$
$8.5 E-02$

$6.9 E-0$

7:0E:D

7.3E-01

2:JE 103

S. $9 E-01$

$1.5 E-01$
$9.4 E-02$

$1 . S E-04$
$6.0 E=05$

$6.9 E-05$
$6.0 E-05$

$1 . P E-O I$
$7.2 E-03$

5. ᄀE :0

1.3E :0

5.8E :00

3.6E-06

2:AE-06

3.9E 00

$3.2 E-02$
$4 . B E-05$
BREAST

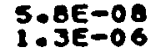

$6.7 E-07$

$1.3 E=00$

$3: 4 E-04$
$1: 3 E-02$

A.2E
$B .0 \mathrm{~B}=04$

$2.9 E=04$
$3.6 E=0.6$

$3 . A E-06$
$1.2 E-0.04$

i. $4 E=04$

$2.6 E-05$
$1.7 E-05$

1.2E=-0

:7YE:

$3.4 E-23$
3

$1 \cdot 3 E-23$

$49 \mathrm{E}-16$

6.7E-14

1.:2E-10

8. $3 E=08$

$5.8 E-05$

$2.0 E-05$

3. $0 E=10$

$4.2 E-05$

$1 . P E=02$
$T Y 0=02$

7:1E-02

7.2E-OI

$6.5 E-02$

$2 \cdot 5 E-03$

$2.8 E-0 l$
$8 E-02$

7. $7 E \rightarrow 02$

$1.2 E=04$
$7.1 E-05$

6.BE-05

$1.9 E-01$
$8.0 E-03$

S.8E :0

:IE OI $1: 3 E$ O:

ITE OI $6: 0 E$ OO

作

.9E-06 $6.9 E-06$

2.66 OI $3 . A E$ OO

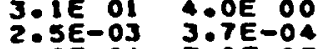


I NHALATION DOSES AT 50 YEARS

(REMUMICROCURIE)

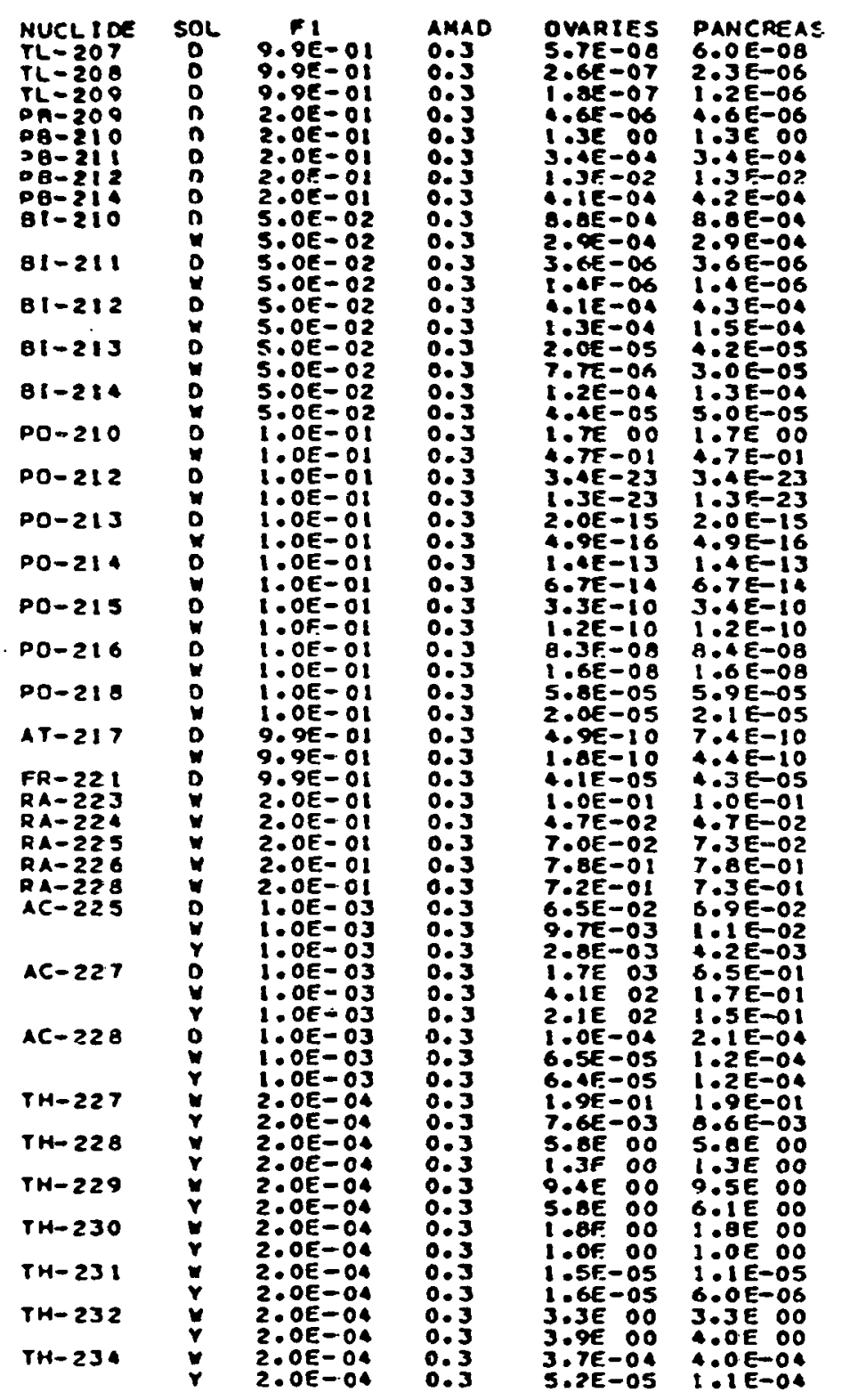

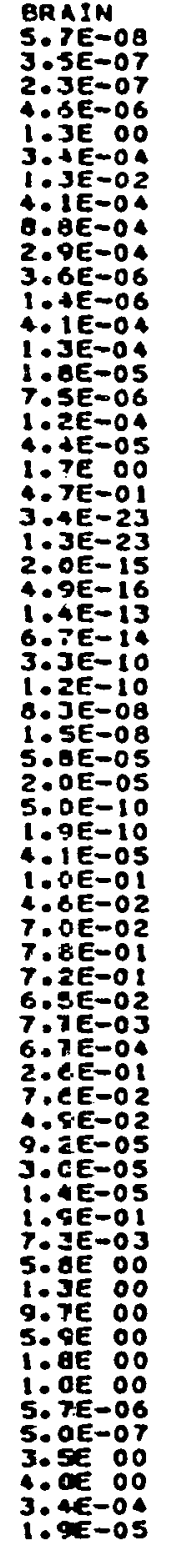

\begin{tabular}{|c|c|c|}
\hline 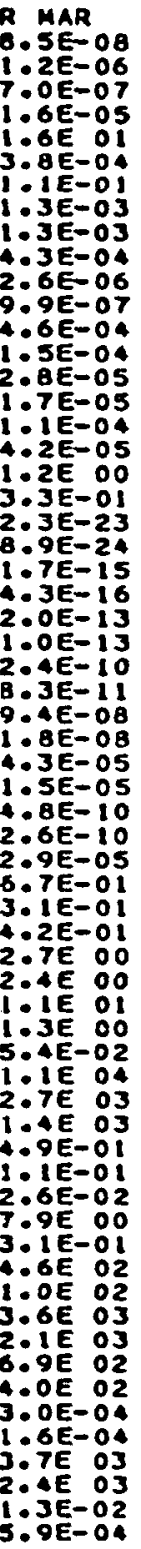 & 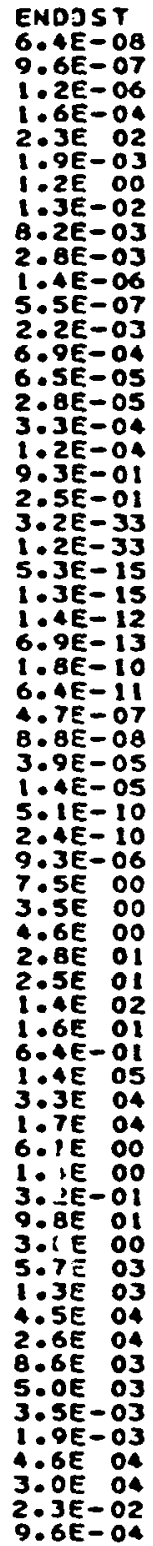 & 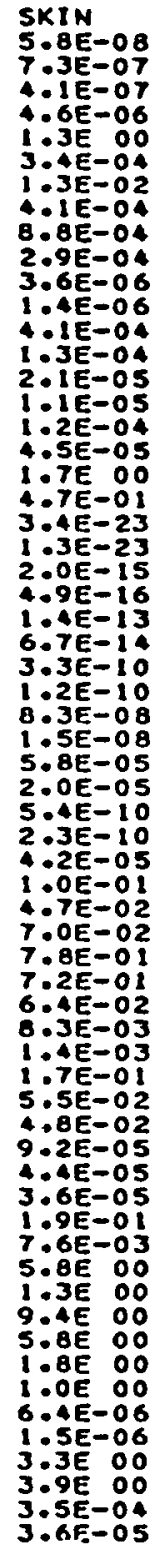 \\
\hline
\end{tabular}

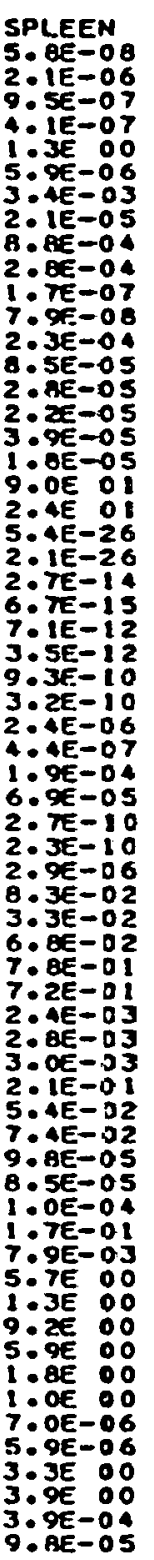

TESTES

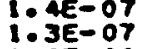
$4: \begin{array}{ll}: 6 E-06 \\ 3 E\end{array}$ 3:A $3 E=02$ \begin{tabular}{lll}
$1: 3 E-02$ \\
\hdashline $1 E=04$ \\
$1 E=04$
\end{tabular} 3. $6 E-06$ $1: 4 E=06$
$4: 1 E=04$ $4: 16=04$ 1.:3e $6=05$ S: $\because: 3 \in=05$

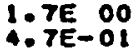
3.:AE-23 $200 E-15$ i. $A E-1$ 6. $3: 3 E=10$ 1. $3 E-0 \circ$ $5: 0 E-05$ $2.0 E-05$ $\because 9 E-10$ $1: Y E=10$ $1: 0 E=01$ 6. $9 E-02$ $7.0 E-01$ 7:2E=01 7. SE-OS S: $6 E-0.4$ $\therefore$ 6. $0 E-05$ $2: 2 E-05$
$8: 8 E-06$

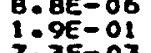
$5.8 \in \mathrm{OO}$ 1.3E S. ᄀe :0 1:ae $: 0$

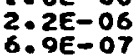
$3.3 \mathrm{BE}$.O 3: 1 KE-04

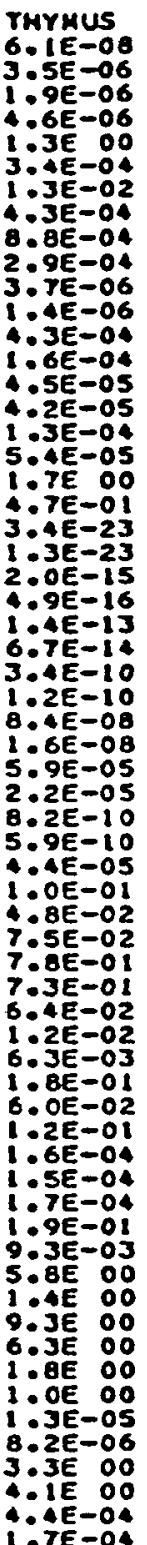
$1-1 E-06$ 2:TE-OT $4.6 E-06 \quad 1: 6 E-06$ 作 -3E-02 $1: 3 E-02$ B. $8 E-04$ : :BE- 04 $2.9 E-04 \quad 2.0 E-04$ $3.6 E-06 \quad 3.6 E-06$ 1.AE-06 1.AE-06 $1.4 E-04$ 1.3E-04 1.4E-05 $0: 1 E-06$ 1.6E-05 $1.4 E-05$ $4.7 E-01 \quad \angle 7 E-01$ $3: 3 E-23 \quad 1: 3 E-23$ $2: 0 E-15 \quad 2: 0 E-15$ 1.AE-13 $1: A E-13$ $6.7 E-14$
$3.7 E-14$
$3: 3 E-10$ $\begin{array}{ll}3.3 E-10 & 3.3 E-10 \\ 1.2 E-10 & 1.2 E-10\end{array}$ (1) $5.5 E-05$ 5.SE-05 $2.0 E-05 \quad 2.0 E-05$ $5.6 E-10 \quad 4.9 E-10$ $2.6 E-10 \quad 1.0 E-10$ $\because: O E-O 1$ T.OE-OI

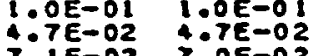
$7.1 E-02 \cdot 7.0 E-02$

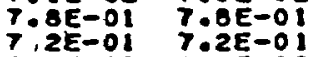
$\begin{array}{ll}7,2 E-01 & 7.2 E-01 \\ 4 E-02 & 6.4 E-02\end{array}$ $\begin{array}{ll}0 E-02 & 0.5 E-03 \\ 0.5 E-03 & 0.5 E-03\end{array}$ $\begin{array}{ll}1: T E-03 & 1.6 E-03 \\ 1: 5 E-01 & 1.3 E-01\end{array}$ $5.0 E-02 \quad \triangle . A E-02$ 5. JE-02 $3.2 E-022$ $5.3 E-05$ 4.5E-05 ::GE-OL $1: 9 E-O 1$

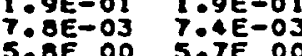
1.JE OO I.JE OD Q.AE OO 9:3E OO S.PE OD S.TE OO

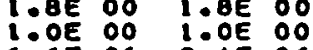
$6.6 E-06 \quad 8 \cdot 4 E-06$ 3.JE OO उ.JE 00 3.9E 00 3.8E 00 $3.6 E-04$
$5.3 E-0 S$
$3: 1 E-04$ 
KIONEYS LIVER

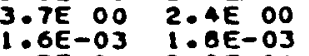

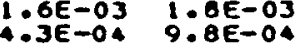
. BE-05 $2.0 E-04$ 9.7E-05 $\frac{1}{3: 0 E-04}$ OE of $9.5 E-02$ $1.9 E$ of 1 . 1 EE-01 $5.3 E$ : $3: 16-02$ :BE O1 $1: 1 E-0$ S.2E 政 $2.5 E$ oO $A: 4 E-02$

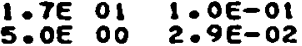
.6E 00 i. $5 E-02$

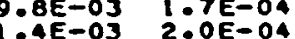
$103 E-04221 E=04$ .TE OO O $3 E-02$

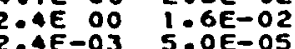
$\because 3 E-04 \quad 5.4 E-05$ $4.6 E-05$ S. $A E-05$

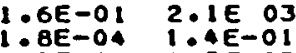
6.3E-05 $1.3 \varepsilon-03$

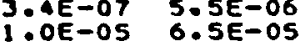
$2,4 \varepsilon-02$ $1: 6 \in E-02$

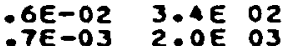
$2 . B E-03$ i: $1 E$ O3

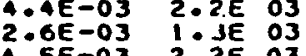
$2: T E-03$ 2.6E-04 5 $2: 0 \varepsilon-06$ 2. $8 \in E-01$ $2.4 E-02$ $1: 3 \in-02$ 1:Be-05 据

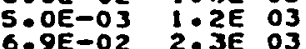
$\begin{array}{llll}6.2 E-02 & 2: 3 E & 03 \\ & & \end{array}$

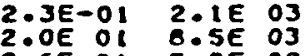
$\begin{array}{ll} & \\ 2 & \end{array}$ 2. $3 E-03$
QREAST $3.9 E-02$ $4.5 E-04$ 5. $3 \varepsilon-09$ 2:AE-O9 $1,1 E-0:$ OOE-Oㄴ 1. $\begin{aligned} & 1 E-01 \\ &: 1 E-02 \\ & 1 E-02\end{aligned}$ $1.6 E-02$ 1. $1 E-01$ $1.0 E-02$ $3.1 E-02$ $3.4 E-02$ $2.9 E-02$ T) $03 E-04$
$3 E-04$ $1.3 E-04$
$9.6 E-02$ $2.0 E-02$ Q. $4 E-05$ $3.5 E-05$
$30-02$ $1.8 E-04$
4 $7.2 E-05$
$7.2 E-0 ?$ 1.2E $-7 E-02$
$A E-02$

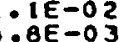
3.OE-OJ $4 . \varepsilon-03$ . $2 E \quad 03 \quad 45 E-03$ $3.3 E$
3 ol $1.7 E-04$ . $9 E-04 \quad 2: 4 E-06$ IE 03 2.3E-O $1.2 E$ O3 $1: 9 E-01$

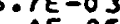
$1: 4 E-05$

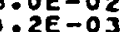
. $1 E-02$ $5.0 E-03$ $3.5 E-02$ i.je-ol 
[RELWHACROCURIEI

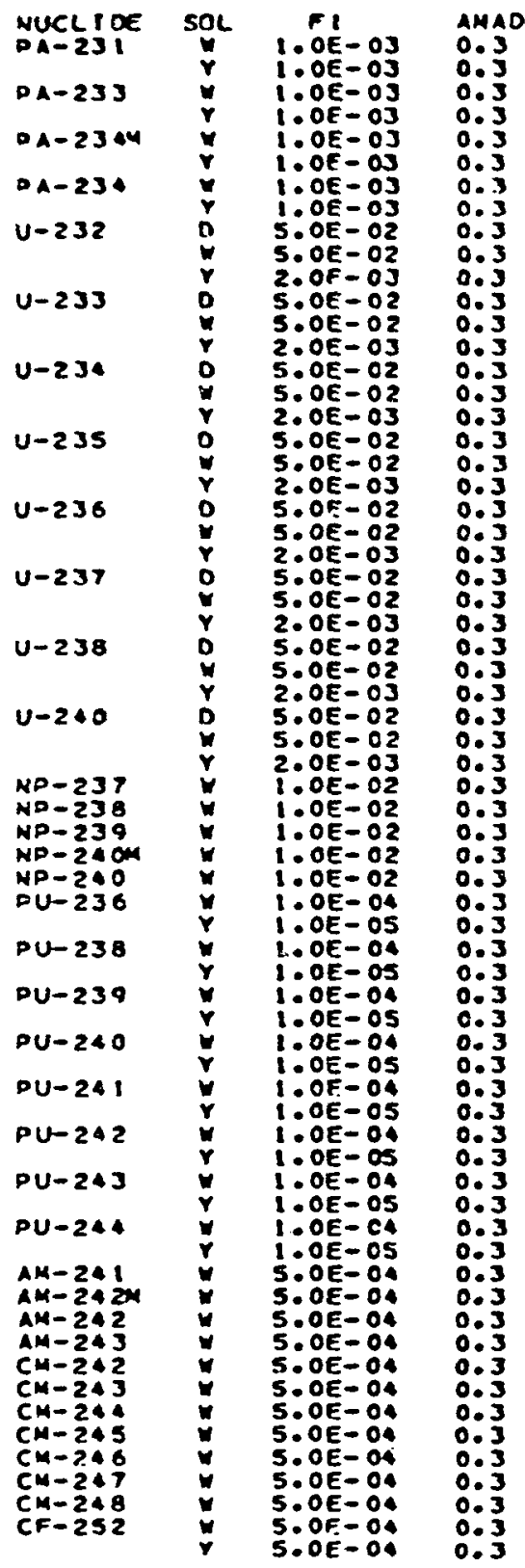

OVARIES PANCREAS

O.AE-02 PANCREA

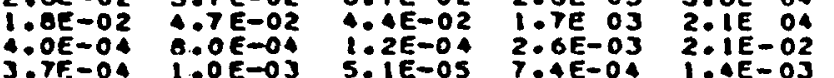

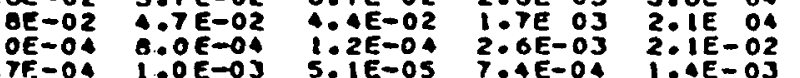

BRAIN

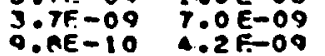

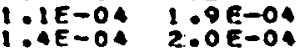

3.ge-01 3.3e-0l

I.2E-DI $1.0 E-01$

YiE-O1 $1: 1 E-01$

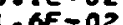

$1.1 E-01$

$3.1 E=02$
$3.6 \varepsilon=02$

$1.1 E-01$
$3.0 \mathrm{E}-02$

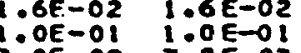

$\begin{array}{ll}3: 0 E-02 & 3.2 E-02 \\ 1.0 E-02 & 4.6 E-02\end{array}$

$1: 0 E-01 \quad 1.0 E-01$

$2.96-02$
$1 . S E-02$
$3.5 E$

$2.9 E-02$
$1.5 E-02$

$2,0 E-042.2 E-04$

$9.5 E-02 \quad 9.6 E-02$

$\begin{array}{ll}2 . T E-02 & 2.8 E-02 \\ 1.5 E-02 & 1: 9 E-02 \\ 1.2 E-05 & 1.1 E-04\end{array}$

(.)

S.AE-05 $6.3 E-05$

- .2E-OJ $2.9 E-04$

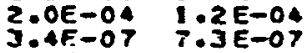

$6.1 E-06$

$3.9 E$ of $3.0 \mathrm{E}-02$

$1.2 E$ O2

$\begin{array}{lll}6.2 E & 01 & 2: 8 E-03 \\ 1.3 E & 02 & 0.01 E-03\end{array}$

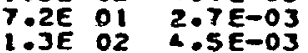

$\begin{array}{lll}1.3 E & 02 & 2.5 E-03 \\ 7.2 E & 01 & 2.750 \\ 3 & 03\end{array}$

2BE :O JOTE

$\begin{array}{lll}1: 2 E & 02 & 5: 1 E-03 \\ 0.8 E & 01 & 3: 2 E-03\end{array}$

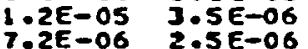

$7.2 E-06$

l.2E :2

\begin{tabular}{ll}
$2.5 E-06$ \\
\hdashline$: E=01$
\end{tabular}

$3.1 E-01$
$306 E-02$

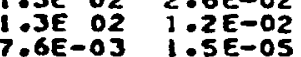

$1: 3 F^{\circ}$ Oz

$: 5 E-05$
$: a E-01$

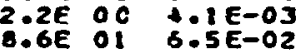

$6.6 E$ il $5.0 E-03$

$\begin{array}{lll}1.4 E & 02 & 5.6 E-02 \\ 3.3 E & 02 & 2.6 \mathrm{E}-01\end{array}$

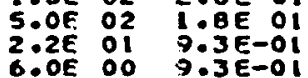

$1: 1 E-01$
IE-05 $\quad: 4 E-04$ 1.AE-OJ $2: 3 E-04$

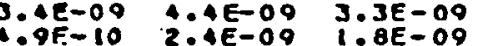

I.JE $E$ - 09

3:SE-OS I:IE-OA 8.2E-05

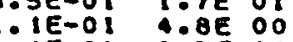

$1.1 E-02$
$1.6 E-02$
$3,0 E-02$

2.7E OO

$2.6 E$ O2 $3.2 E=05$

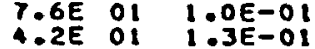

i:SE ol $1: 1 E-01$

$\begin{array}{lll}0.2 E-01 & 1.3 E & 01 \\ 1.2 E-01 & 6.06 & 00\end{array}$

$3.1 E-02$
$1.6 E-02$

2.BE OO

45 OI

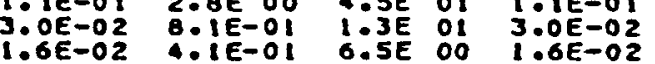

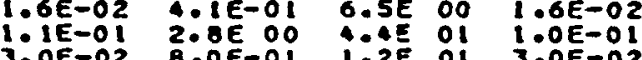

30

1:2E O1

2.TE OO $4: 3 E$ OI

$3.0 E-02$
$2.2 E-02$

$2.9 E=02$
$1.5 \mathrm{E}-02$

3:OE-OI $6.2 E$ :

$2.9 \mathrm{E}-02$
$1.5 \mathrm{E}-02$

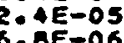

$1 . T E-O 3$

$1.7 E-D 2$
$2.4 E-03$

I. $2 E-04$

$2 \cdot 2 E-04$

$2: 2 E-04$

: $T E-02$

B. $6 E-01$

II $9.6 E-02$

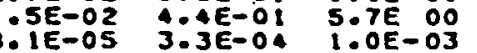

$2: 7 \in-02$

$8.5 E-05$
$2.8 E-0.5$

$\begin{array}{ll}8: T E-05 & 2.2 E-04 \\ 4: 1 E-05 & 5.7 E-05\end{array}$

$1.6 \mathrm{E}-05$
$5.1 \mathrm{E}-02$

$4.8 E-06$

$41 E-05$ 5.0E-02

$9.8 E-03$

$5.16-02$
$9.6 E-05$
3.05

S. $1 E=04 \quad 060-0.03 \quad 3: 1 E-05$

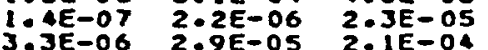

$3.3 E-06$

$2.9 E-05$

2. $1 E-04$

$Y .5 E-07$
$Y, 1 E-06$

.9E-03

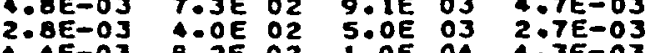

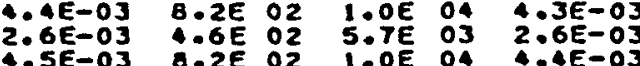

(.5E-03

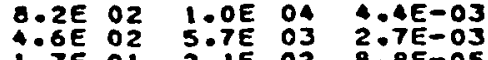

I.TE OP $1: 1 E$ O2 $8.8 E-05$

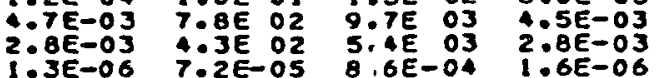

$0.3 E-0.6$
$1.3 E-08$

7. $2 E-05$

2. $2 E-01$

i: $3 E-02$

7.7E 02 $4.5 E-04$

$1.7 E-07$
$1.5 E-01$

5. $3 E$ OJ

10
$1: D E-01$

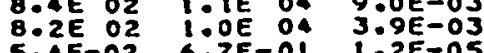

$1: 2 E-05$

5. $4 E-02$ 6. $6 E-01$

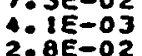

$1.1 E$ OA $4.25 E-02$

1
$1: 1 E=03$
$2.0 E-02$

S.:AE OJ $109 \mathrm{O}-03$

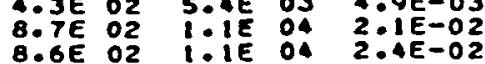

$3.3 E-02$
$3: 0 E-01$

$1,1 E-01$

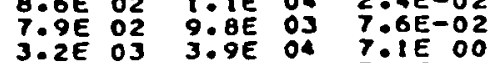

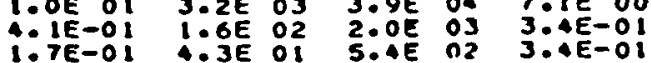

SPLEEN TESTES

$\begin{array}{ll}3.4 E-02 & 2.0 E-02 \\ 6.6 E-0.04 & 6.3 E-0.05\end{array}$

$3.6 E-04$ 3. $3 E-05$

$\begin{array}{ll}6.7 E-09 & 3.1 E-09 \\ 3.8 E-09 & 2.0 E-10 \\ 10 & \end{array}$

(1)

$3: T E=04$ 1:1E-05

$\begin{array}{ll}9.5 E-02 & 9.5 E-02 \\ 2.0 E-01 & 8.04 E-02\end{array}$

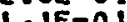

3.1E-02

B. $4 E-02$
$1.1 E-01$

$1.1 E-01$
$3.11 E-02$
$1.0 E$

$\begin{array}{ll}1 E-01 & 1: 1 E-01 \\ 3.0 E-02 & 300 E-02\end{array}$

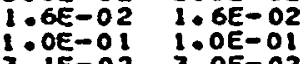

$1.4 E-02$
0

$\begin{array}{ll}1: 9 E-02 & 2: 9 E-02 \\ 1:-9 E-02 & 1: 5 E-02\end{array}$

$1.0 E-0.04$
$1.9 E-0.8 E-05$
$9.5 E-05$

$96-02 \quad 9.6 E-02$

$\begin{array}{ll}1: B E-02 & 2.7 E-02 \\ 1: 9 E-02 & 1: 4 E-02\end{array}$

$\begin{array}{ll}1: 1 E-04 & 20 \\ 30 E-05 & 05 \\ 0 E-05 & 707 E-06\end{array}$

$5.0 E-05$ 5.2E-06

$6.2 E-02$
$2.6 E-04$
1.00

$1.3 \mathrm{E}-02$
$\mathrm{~T}, \mathrm{OE}-03$

$1.00-04$
$6.1 E-07$

$2.0 E-05$
$9.5 E-03$

3:1E-05

3. 7E- 06

$4.6 E=03$

$2 \cdot 0 E=03$

$1.9 E$ OI

6.2 of

2. $6 E-03$

$3: 7 E=03$

7.2E OI

$1:$ JE 02

2.2E 00

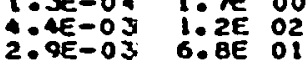

3.1E-DG

6. BE OI

1. $6 E-01$

$5.7 E-06$
$1.2 E$
62

OOE-02 $1: 3 E$ O2

$1.5 E-05$

$: .3 E$
$7.6 E-03$

i.

2.JE O2

$4.9 E-03$
$2.0 E-02$

6.6e of

$2.4 E=02$

6. $\triangle E E$ Oa

I:AE 02

5.JE 02

$2.2 E$
$6.0 E$
THYMUS THYROID UTERUS.

$\begin{array}{lll}2: 4 E-02 & 3.5 E-02 & 2: 0 E-1.2 \\ 1: 1 E-02 & 3: 1 E-02 & 1: \Delta E-0.3\end{array}$

$1.1 E-03$ 3.0E-04 1.0E-B.

$9 . B E-00$ 5.2E-00 3.SE SEI

$\begin{array}{lll}2.0 E-09 & 2.2 E-09 & 6.5 E-12 \\ 2.6 E-04 & 0.0 E-05 & 0.0 E-2.5\end{array}$

$2.6 E-04$ 8.2E-05 $7: 9 E-r 5$

3.JE-OI $3.3 E=01 \quad 3: J E-5$

3.1E-OI 1:OE-OI

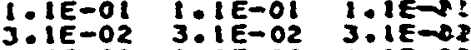

$1.6 E-02$ 1.6E-02 $1.6 E-22$

$1: 1 E-01$
$3.0 E-02$
$3.0 E-02$
$1.0 E-02$

$1.6 E-02 \quad 1.6 E-02 \quad 1.6 E-02$

$\begin{array}{lll}3: 0 E-01 & 1: 0 E-01 & 1.0 E-21 \\ 30 E-02 & 30 E-02 & 300 E-02\end{array}$

$6.6 E-02 \quad 2.6 E-02 \quad 1.7 E-22$

$2.9 E-02 \quad 2: 9 E-02 \quad 2: 9 E=-2$

$1.4 E-04$ I: $3 E-04$ 1:JE $\rightarrow 0$

3.2E 04 OAE

.5E-02 9.5E-02 9.5E-22

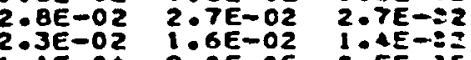

1.1E-04 $9.0 E-05$ B.SE-5

$\begin{array}{ll}7.5 E-05 & 2: 5 E-05 \\ 7.6 E-: 5\end{array}$

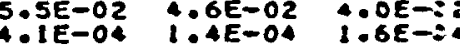

$1.5 E-04 \quad .0 \mathrm{E}-05$ 6.5E $\rightarrow$

$3.0 E-06 \quad 3.8 E-07 \quad 1: 1 E-07$

$3.3 E-05$
$1.5 E-02$

2.7E-03 $2: 7 E=03 \quad 2.6 E=33$

$4.3 E-03 \quad 2.3 E-03 \quad 4.3 E-63$

$2.6 E-03 \quad 2.6 E E-03 \quad 2.6 E-63$

2.7E-03 2:BE-03 $2: 6 E-C 3$

$7.0 E-05 \quad 6.3 E-05 \quad 5.2 E \rightarrow S$

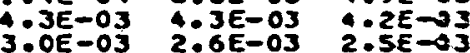

$4.4 E-06 \quad 1.8 E-06 \quad 1.8 E-36$

$3 . A E-06 \quad 6.6 E-07$ 9.1E-37

AE 01 1.IE-01 $5.9 E \rightarrow 3$

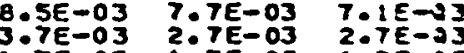

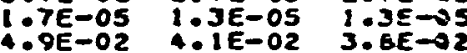

$\because 1 E-03 \quad 4: 1 E-03 \quad \because 1 E-03$

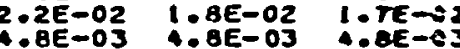

$2.3 E-02 \quad 1: 8 E-02 \quad 1: 8 E=2$

$9.3 E-02 \quad 7: 2 E-02 \quad 5: 7 E-02$

3.9E-OI $2: 2 E-O 1$ 1:TE-Ai 
I NHALAT ION DOSES AT 50 VEARS

(REM/MICROCURIE)

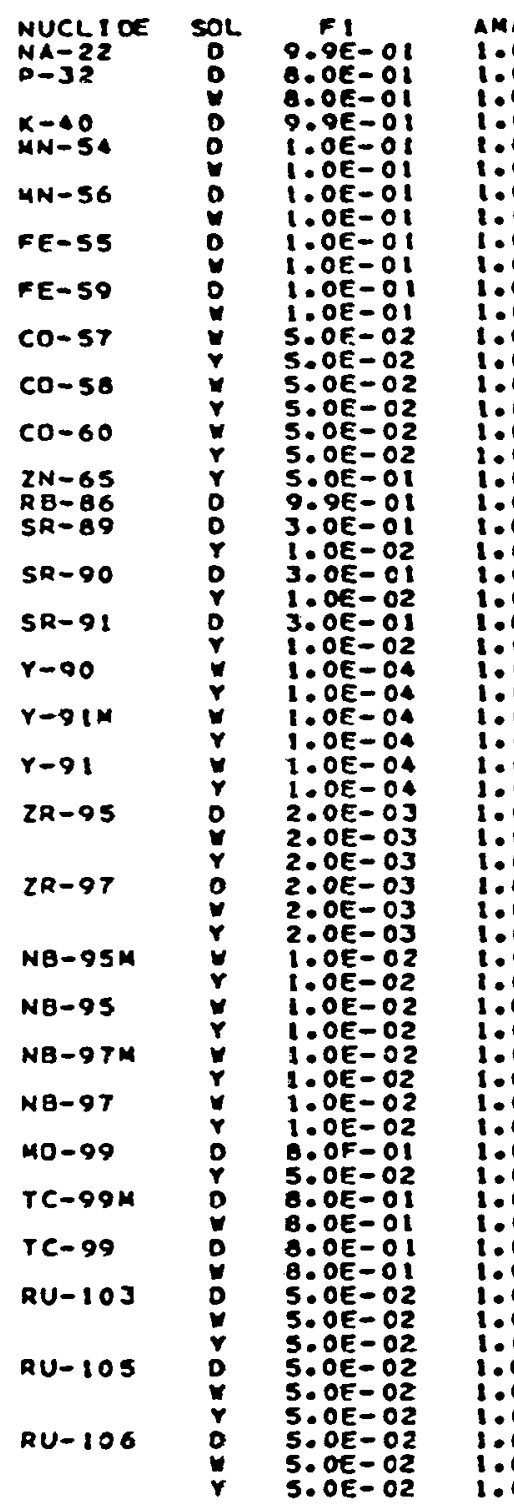

EFFECT: LUNGS

\section{SI YALL ULI Y}

LLI WALL AORENAL

ZRALL $\begin{array}{ll}6.0 E=03 & 9: 1 \mathrm{E}-03 \\ 1.0 E-02 & 1.0 \mathrm{E}-0\end{array}$

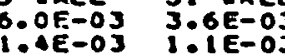
$1.0 .0 E-0.03$
$1.0 E-0.03$ I: JE- 03 5.OE-03

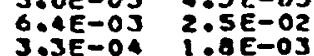
$3.3 E-04$
$3.0 E-04$
$3.6 E-0 J$ $\begin{array}{ll}2.6 E-03 & 2: 1 E-03 \\ 1: 3 E-0.03 & 100-03\end{array}$

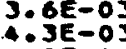
$5.2 E-0.4$
$3.0 E-0.0$ .OE-0. $1.2 E-03$ 1:2E-0J $1: 3 E-02$ i.2E-04 $6 . A E-03$
$7.0 E-0.5 E-03$ $1,2 F-02 \quad 5.3 E-02$ $2.6 E-03 \quad 1.5 \mathrm{E}-02$ $\begin{array}{ll}8.6 E-03 & 6.3 E-02 \\ 6.2 E-03 & 3.0 E-02\end{array}$ $\begin{array}{ll}6.2 E-03 & 3.0 E-02 \\ 1.0 E-02 & 6 . j E-02\end{array}$ $2.0 E-01$ I. $3 E=00$ $1.9 E-02 \quad 7 . B E-02$
$80 E-03 \quad:-2 E-02$ $\begin{array}{ll}8.0 E-03 & 1: 2 E-02 \\ 6: 3 E=03 & 8.02 E-03\end{array}$ $\begin{array}{ll}6.3 E-03 & 8.2 E-03 \\ 4.1 E-02 & 3.1 \in-01\end{array}$

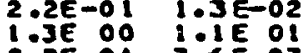
$\begin{array}{ll}8.2 E-O A & 3.6 E-03 \\ 1 . B E-03 & 1.1 E-02\end{array}$ $7.5 E-03 \quad 3.9 E-02$ $\begin{array}{lll}8.0 E-03 & 1.2 E-02 \\ 3.1 E-05 & 1.9 E-0.04\end{array}$ $3.2 E-02 \quad 2: 0 E-O 1$ $4.3 E-02$
$3: 3 E-02$
$3.17 E-01$ $\begin{array}{ll}1.5 E-02 & 6: 9 \varepsilon-02 \\ 2.3 E-02 & 1.5 E-01\end{array}$ $\begin{array}{ll}2.3 E-02 & 1.5 E-01 \\ 2.2 E-03 & 7.9 E-03\end{array}$ 3. $2 E-03 \quad 2.2 E-02$ 2:1E-0J $1: 1 E=02$ $4.7 E-03 \quad 2.1 E-02$ $\begin{array}{ll}5.7 E-03 & 3.1 E-02 \\ 2.1 E-06 & 1.5 E-05\end{array}$ $1.3 E-04 \quad 1.7 E-05$

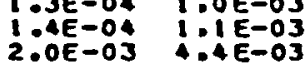
$3.8 E-03 \quad 1: 9 E-02$ $3.2 E-05 \quad 9.0 E-05$ $\begin{array}{ll}3.6 E-05 & 1.9 E-04 \\ 100 E-03 & 1.3 E-03\end{array}$ $\begin{array}{ll}\mathrm{B}: 3 E-0 & 03 \\ 2.9 E-03 & 6.2 E-02 \\ 0.9 E-03\end{array}$ $\begin{array}{lll}6: 3 E-0.53 & 3.7 E-02 \\ 8.05-03 & 5.9 E-02\end{array}$ $3.1 E-04$
$5.2 E-04$ $5.9 E-02$
$1.5 E-03$ $3 . A E-03$

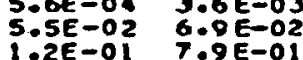
(at)-。 2.:E=03 $2: 1 \mathrm{E}=04$ $5.2 E-03 \quad 2.7 E-03$ $1.0 E=0122.4 E-02$ 3. $2 \varepsilon-03$ $1.2 E-0 J 1.5 E-03$ \begin{tabular}{ll}
$1.2 E-03$ \\
\hdashline $6 E=03$
\end{tabular} $8.1 E=04$
$1.4 E-03$ 1. $1 E-03$ 1. 1 1E-OS 1:A $A=03$ $\because \because 2 E=03$ $8.8 E-03$
$1.0 E-03$ $1: 6 E=03$ 1.AE-OJ 3.9E-03 S.AE-O $9.1 E=0$ $2.0 E-03$
$2.4 E-03$
$.3 E-06$ $2: 1 E-06$ $6.7 E-05 \quad 4: 6 E-05$ B. 2E-05 $7: 0 E-04$
$0.9 E-04$ $1.1 E=04$ $6: 0 E-05$
$9: 3 E-03$ 2. $1 E-03$ l.TE-03 $3 . A E-04$ 2. BE-04 $\begin{array}{ll}2.9 E-02 & 2.9 E-02 \\ 1.2 E-02 & 1.5 E-02\end{array}$

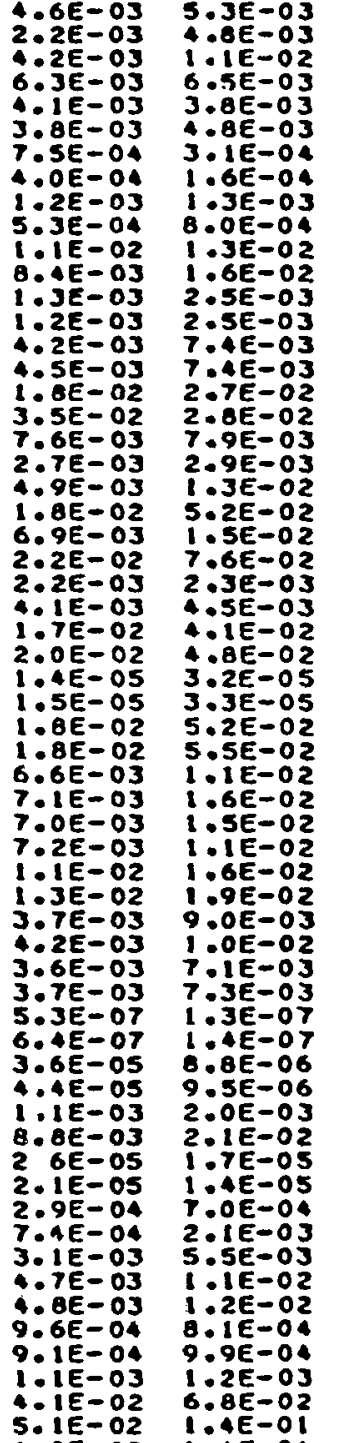

1: $: B E=03$ 6.

T. $2 E-03 \quad 1 \cdot A E-03$ $5.5 E-05$ 2.0E-05 $\begin{array}{ll}2 . D E-03 & 2.0 E-03 \\ 6 . T E-04 & 6.7 E-04\end{array}$ $1.5 E-02$ $\begin{aligned} & 6: B E=03 \\ & 1.0 E-03\end{aligned}:=2 E=0$ $2 . B E-03 \quad 2.2 E-04$ $6.0 E-03 \quad 9.0 E-O$

$1: 1 E-01 \quad 1: 1 E=02$

5

1: $V E=03$ 3. $2 E-05$ $9.0 E-04$ $1.2 E E-04$ 3. $x E-05$ 2:1E-06

7. $0 E-06$

$\because: 0 E-O S$

$1: 1 E-02$

S.TE-OJ

$4 \cdot 9 \varepsilon=04$

2: $T E-0$

$2.5 E-04$

$2 \cdot 7 \in=04$

$2.5 E-03$

3.6E-07

$1: A E=05$

6. BE-04

1:SE=D4

$1: 3 E=05$

$1.5 E-04$

$2 . R E-03$

1: $1:=-03$ $6.11-05$ $5.4 E-05$ $5.4 E-02$
$1: 6 E=02$
$8.8 E-03$
5. 2E-03

3. 2E $=05$

$9.0 E-O 4$

$1.96-0$
$6.3 E-05$

$3.9 E-05$

1. $2 E-06$

A. $2 E-04$

:.1E-03

$1 . A E=03$
$70 E=04$

A. SE-O4

2. $0 E-04$

9. $3 E-05$

7. $6 E-04$

5. 5E-OB

3. $A E=06$

5. OE-O

$1.3 E=04$
$7.6 E=06$

$3 . \Delta E=06$

i. SE-04

2. $A E-03$

40 AE 04

$2: 7 E-05$

5. $4 E-0$

$1: 5 E-02$
$\because .6 E-03$

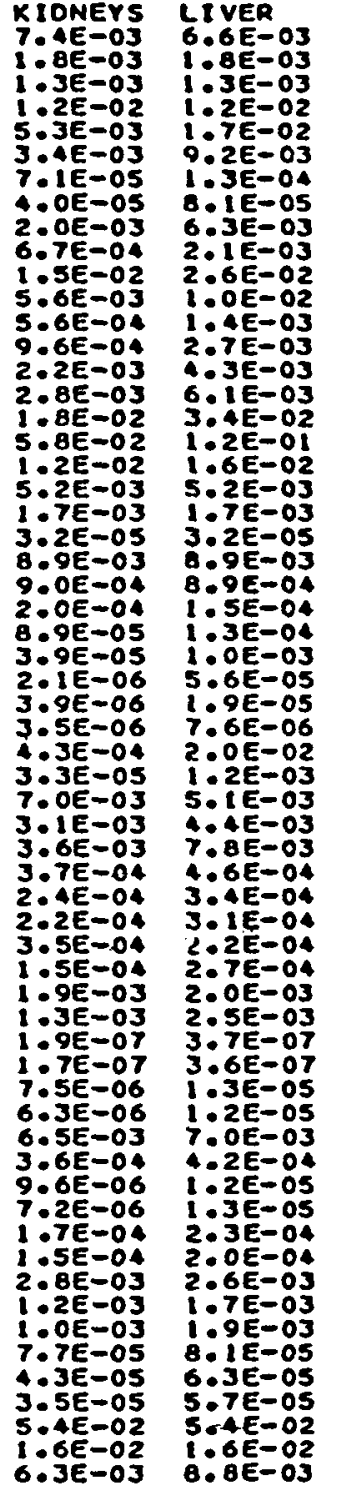

BREAST

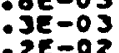
. 3. $3 E-03$ $6.2 E-05$ A.JE-0S $6: 7 E=04$ $1.3 E-02$ $6.2 E-04$ $1.4 E=03$ 3. $5 E-03$ $6: 9 E=02$ $5.2 E-03$ 3.2E -05 O 1. 0 E-O4 B.SE-OS 2.1E-06 $4 . A E=06$
$4.0 E=06$ $4.3 E-04$ 3. AE $3.5 E-03$ $4.6 E-03$ $2.6 E-04$ $2.1 E=04$ $1.6 E=04$ I. SE -03 $2.3 E-07$ 8 . $7 E-06$ T.IE-06 $5.2 E-04$ 1.1E-0 B.:0E-06 2.6E-03 $1.3 E-03$ $1.2 E-03$ $4 . J E-05$ $3.6 E-05$ S.AE-02 $1.6 E=02$
$6.9 E-03$ 
I NHALATION DOSES AT SO VEARS

(REM/AICROCURIE)

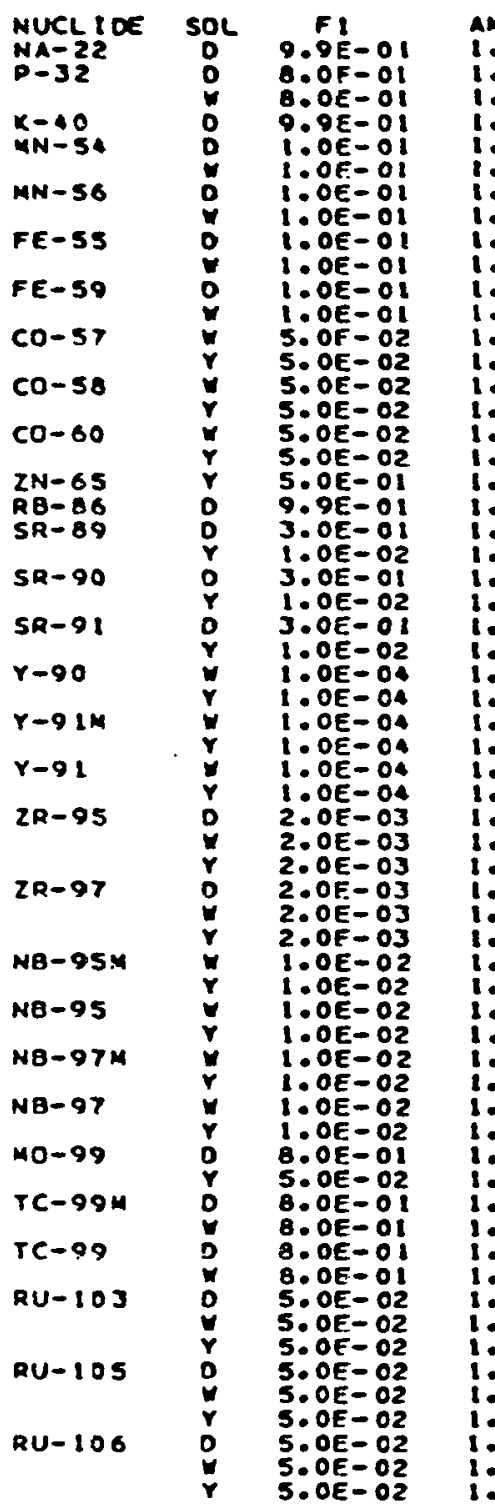

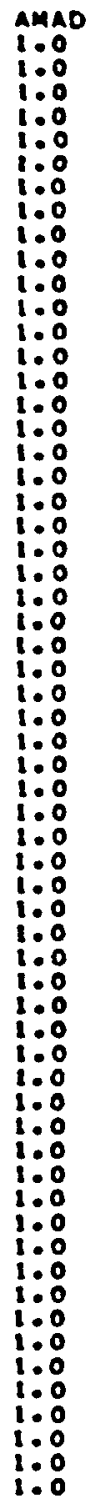

OVARIES PANCREAS gRAIN

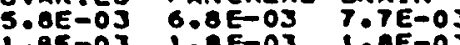
$1,0 E-03$ I.BE-03 I.OE-OJ i. MAR

ENOOST $1.3 F-03$
$1.2 E-02$ $\begin{array}{ll}1.3 E-03 \\ 1.2 \mathrm{E}-02 & 1.3 \mathrm{JE}-03 \\ 1.2 \mathrm{E}-02 & 1.03\end{array}$ 2. $6 E-03$ i: 8.2E-05 $9: 3 E-05$ A.8E-05 $\begin{array}{lll}3.7 E-05 & 7.3 E-05 & 1 \\ 1.7 E-05 \\ 1.0 E-03 & 2: 0 E=-03 & 2\end{array}$ $1.9 E-03$ GE-O4 $1 \cdot 1 E-02 \quad 1: A E-02$ $5.5 E-04 \quad 1.0 E-03$ $\begin{array}{lll}A 5 E-04 & 2.7 E-03 \\ 2.4 E-0.3 & 0.7 E-03\end{array}$ $2.3 E-03 \quad 6.2 E-03$ $1.3 E-02 \quad 2.3 E-02$ $7.0 E-03 \quad 1.5 E-02$ $\begin{array}{ll}5.1 E-03 & 5: 1 E-03 \\ 1 .: E-03 & 1.7 E-03\end{array}$ $3.2 E-05 \quad 3.2 E-05$ $8.9 E-03$ $9.0 E-04$
$2.5 E-0.04$ $0.9 E-03$
$9.0 \mathrm{E}-04$ $2: 1 E=04$ $2.2 E-041.5 E=04$ $2.1 E-06 \quad 2.1 E-06$ . $1 E-06 \quad 8.9 E-06$ $\because: 3 E-04 \quad 9: 1 E=06$ $3.3 E-05$ T.2E $\quad \forall 1 E-05$ $3.1 E-03$ $7.1 E=0$.
$3.1 E$ $4.9 E-03$
$8.3 E-03$ A.TE-OA (2.5e $6.5 E-04$
$7.0 E-04$
$3.0 E-04$ $2: 1 E-042.4 E-04$ $.8 E-03$ 2. $1.6 E-03 \quad 2.7 E-03$ $\begin{array}{ll}0.4 E-08 & 4.2 E-07 \\ B .4 E-0 B & 4.2 E-07\end{array}$ $5.4 E-08$ $5.0 E-06$
$3.6 E-06$ $1: 2 E-07$
$1.6 E-05$ 1.6E-05
$1.6 E-05$
0.05 - 1040.3 $3.6 E-04$
$9.2 E-06$
6 $6.0 E-06$
$1 . T E-0.7 E-05$
1 1.re-04 $1.7 E-04$
$1.5 E-04$ $2.6 E-03 \quad 2.5 E-03$

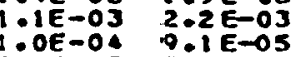
. $1 E-05$ $7: 1 E-05$
$T: 1 E-05$ $6.2 E-05$
$5: 3 E-02$ $\begin{array}{ll}5.3 E-02 & 5.3 E-02 \\ 1: 5 E-02 & 1: 6 E-02 \\ 5.0 E-03 & 9.3 E-03\end{array}$ $2.3 E-02$ :.6E-02 1.0E-05 $2.05-03$ $1.2 E-02$ 3.

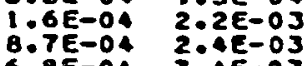
. BE-04 3.AE-OJ $\begin{array}{ll}1: 1 E-02 & 1.6 E-02 \\ 1: Y E-02 & 6.4 E-02\end{array}$ I.AE-02 $\begin{array}{ll}3: 2 E=03 & 2: 0 E-02 \\ 1: T E=03 & 2: 1 E-02\end{array}$ $3.2 E-05$
$8.9 E-03$ 政. 1. $6 E-04$ OE-O3 2.1E-06 5.7E-05 6.2E-07 ?:5E-06 $3: 2 E-04$
$2: 6 E-05$ $1.2 E-02$ $2.8 E-03$
$B .9 E-04$ 3. $9 E-04$ $3.5 E-05$
$9.0 E=05$ 3. 0 - 05 $7.3 E-04$
$2: A E-04$ $6.4 E-0.8$
$3.4 E-0.8$ $3.4 E-08$
$3.2 E-06$ $1: 2 E-06$ 2.TE-05 3. $1 E-06$ $1: 3 E-04$ $2.65-03$ $3.0 E-04$
$5: 5 E-05$ $1.6 E-05$ 1.7. $5.4 E-02 \quad 5 . j E-02$

$6.6 \mathrm{E}-03$

I:SE-02

$9.1 E-03$

B.
BE
$3 E-05$

$3.5 E-05$
$1.9 E-03$

$6.4 E-04$

$3.55-03$

$5.8 E-04$

$1: 6 E-03$
$1.6 E-03$

2.5E-:3

$4.9 E-02$

3. $1 E-02$

6.:E-:04

2:AE- OI

6.3 $1.00=05$

$5.7 E-05$

$1.5 E-05$
$3.6 E-06$

$\begin{array}{ll}2: 1 E-02 & 2: 1 E-02 \\ 1.2 E-03 & 1: 2 E-03\end{array}$

$1.0 \mathrm{E}-02$

$5: 2 E-02$

8.1

8.: $7 E-03$

5.8E-04 $4.7 E=04$

$2.7 E-04$
$5 . B E=0.4$

2.1E $=04$ 1.JE-OJ

2:5E-03

$2.4 E-07$
$2.0 E-07$

9.2E -03

$1.9 E-03$

1.8E-07

$\begin{array}{ll}1.0 E-05 & 7.6 E-06 \\ 7.0 E-06 & 5.2 E-06\end{array}$

$1: A E-03$
$2: 1 E-04$

1: $3 E-05$

2. $1 E=03$

6. $7 E-06$

7. SE-06

$1: 5 E-04$
$1.5 E-04$
$1.5 E-03$

9.0E-04

$8.6 E-04$
$5.5 E-05$

$3.2 E-05$
$2.5 E-0 S$

$5.2 E-02$
$1.5 E=02$ A.TE-03

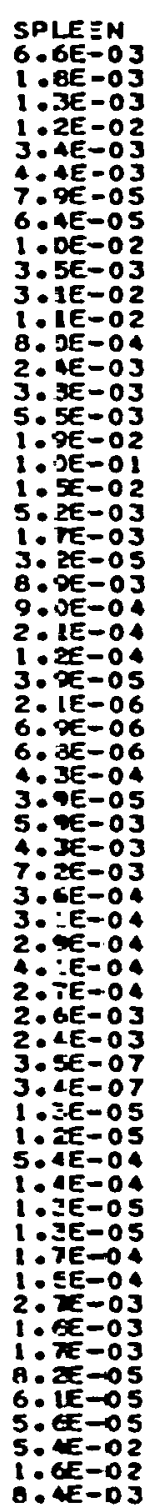

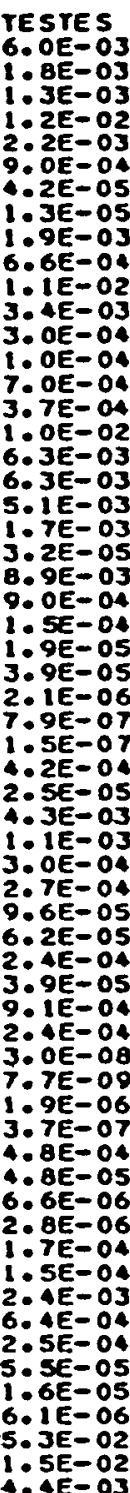

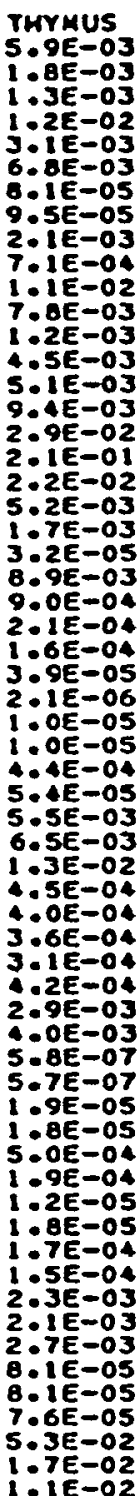

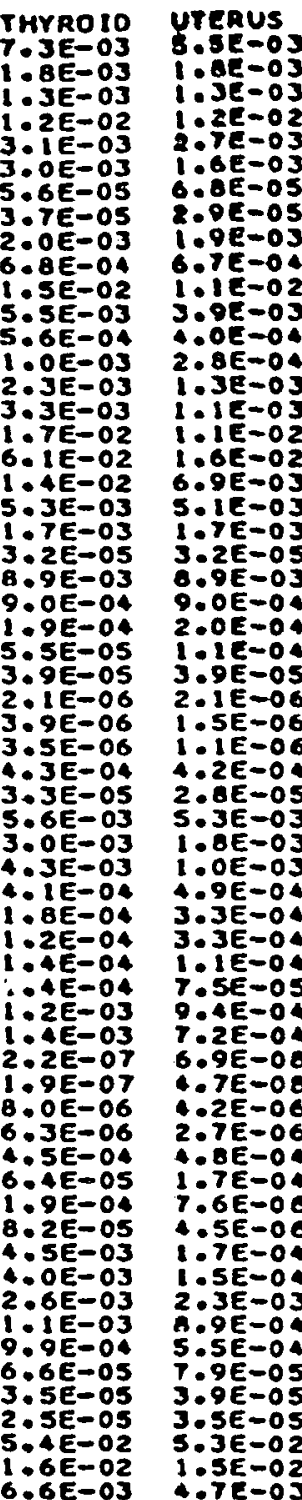


INHALAT ION DOSES AT 50 VEARS

(REMTMICROCURIE)

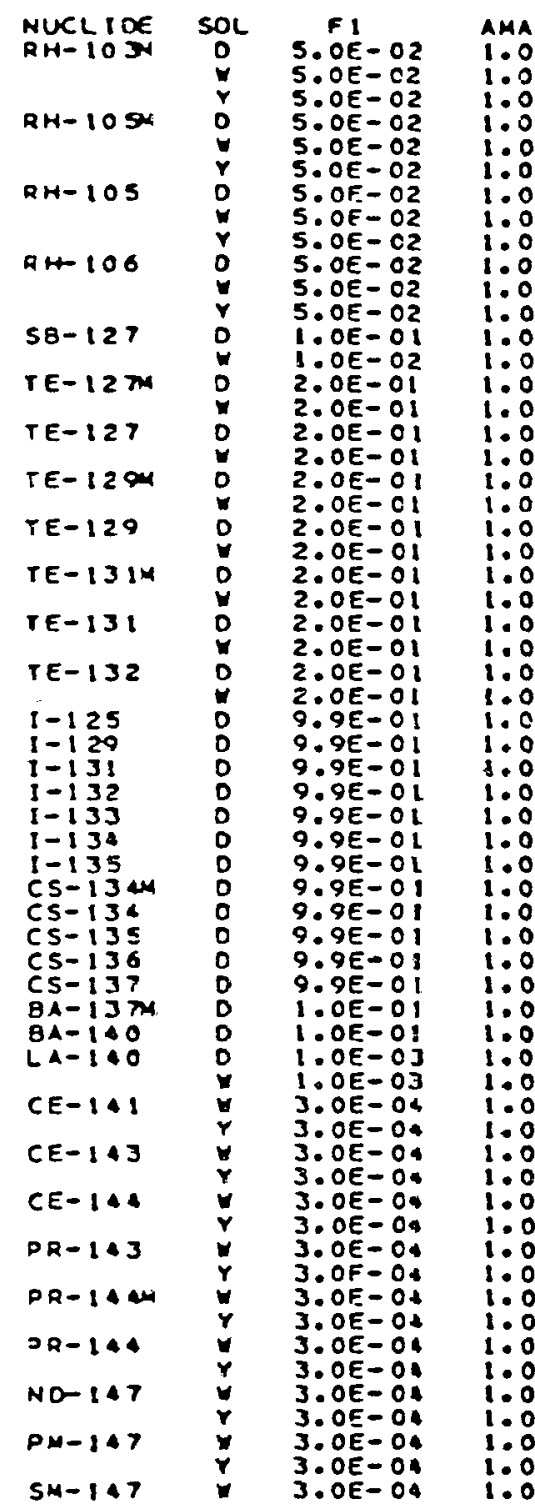

EFFECT. LUNCS 5.3E-06 3.6E-05 :.2E-06 $6.6 \mathrm{E}-05$ $4.3 E-072.9 E-08$ 3.0E-OT $6.2 E-0$ ? $8.5 E=04$ O.IE-04 $2 . \theta E-06$ , $B E-06$ 2. $2 E-03$ $1: 4 E-02$ $2,1 E-0$ $3.7 E-04$
$9.0 E-03$ $2.3 E-02$ : $3 E=04$ $3.3 E-03$ : $2 E-04$ $1.2 E-04$
$3.5 E-03$ $7.4 E-03$ $1: 8 \mathrm{E}-01$ 3. $3 E-02$ $3.9 E-0$
$8.6 E-03$ $1.5 E-04$ $4.6 \varepsilon-05$ 4 AE-03 $7: 0 E-003$
$3: 0 E-02$ $3: 0 E-02$
$1: 1 E-00$ 3. $2 E-03$ $4.7 \mathrm{E}-03$ $8.2 E-03$
$8 . B E-03$ 3.6
$3.1 E-0.03$

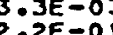
3.TE-01

$7: 9 E-03$ $3.5 E-05$
$3.7 E-05$ $7.7 E-05$
$8.1 E-05$
$6.2 E-003$ $6.2 E-03$ $3.66 E-02$
$3.9 E-02$ $3.9 E-02$
$7.5 E$ OI
SI WALL

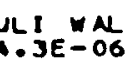

LLI WALL

ADRENAL

a YALL

$1.9 E-06$

$1: 1 E=06$

$8.4 E-0 Y$
$2: 2 E-07$

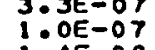

9. $A E-O B$

5. $\begin{array}{ll}\text { 9E-OB } \\ \text { TE }\end{array}$

$\forall: 1 E-07$
$Y T E-07$ B.oE-O?

$\therefore 7 E-O B$

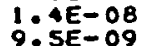

2: $1 E-07$

1. $1 E-07$

(1)

:.IE-03

$1.5 E-06$
$1.8 E-06$

$2: 3 E-08$
$1: B E-0 B$

$9.5 E-09$

5. $9 \mathrm{E}-04$

$3: S E-03$
$2: D E$

. $3 \mathrm{E}-\mathrm{O} 3$

$4.4 E-05$

$3.9 E-05$

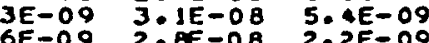

$45 E-09$

5. $0 E-08$ 3.6E-09

$\begin{array}{ll}.8 E-04 & 1.2 E-03 \\ 2 E-03 & 2.0 E-03\end{array}$

5. $6 E-0$.

$2.6 E-03$

$\mathrm{A}: 3 E-03$
$\mathrm{i} 1 \mathrm{I}-02$

$9.7 E-10$

2. $T=O B$

$9.8 E-10$

$20 \mathrm{C}-\mathrm{O} 2$

QE -04

7. $0 E-04$

6. $B E-03$

$2.3 E-04 \quad 6.8 E-04$

$2.7 E-04$
$1.6 E-03$
$2.0 E-0.04$

2.1E-02

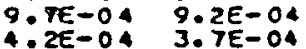

$2:$ TE-05 $2: 7 E=05$

$8.6 E-04$
$1.5 E-02$

$\begin{cases}1 E-0 \\ : 6 E-03\end{cases}$

$2.7 E-03$

$1 . A E-02$

$4.0 E-02$

$8.0 E-06$
$1.6 E-0 J$

1. $5 E-03$

$7: 1 E-04$
$7: 3 E-006$

$2.0 E-05$
$3.0 E-03$

$5 \cdot 2 E-06$

$\begin{array}{ll}0.8 E-06 & 1.9 E-06\end{array}$

BE- 03

$5.3 E-03$

$9.6 E-03$

5: $1 E=04$

$1.2 E-04 \quad 4.0 E-05$

$3.1 E-06$

$1.4 E-03$
$3: 3 E-03$

$\begin{array}{ll}2.5 E-0 J & 2.3 E-03 \\ 7: A E-05 & 3: 8 E-05\end{array}$

$1.7 E-06$
$1.7 E-06$

$1: 3 E-02$

:SE-03

I. $4 E-06$

:AE 05

$2.9 E-02$
$5.2 E-05$

$\begin{aligned} & 1: S E=03 \\ & 7.6 E=05\end{aligned} \quad 1: 1 E=03$

5.6E-0S $8.6 E-05$

$3.4 E-04$

$3.4 E-04$
$0.9 E-05$

$3: 5 E-05$

A. $1 E-05$

$5.2 E-05$
$1.9 E-05$

$8.4 E-05$

:.0E-04

3. $8 E-04$

$5.9 E-05$

$2.0 E=05$
$B . T E-05$

$1: 3 E-05$
$8: 3 E-05$

$1.2 E=04$
$3.6 E-05$

A. $2 E-05$

$8: 1 E-05$

$1,1 E-05$ 9.6E-

i: $:$ SE -05

$1.4 E-05$
$5.1 E-02$
.

$\therefore 6 E=03$

$4.6 E-03$
$7 . T E-03$

$2.3 E-03$
$5: 0 E-03$

$2.3 E-03$
$5.3 E-03$

$8.1 E-03$
$3.4 E-02$

$3.4 E-0$

$1.5 E-02$

:.OE-OT

\begin{tabular}{ll}
$3.8 E-08$ \\
\hline
\end{tabular}

$41 E-07$

$4: O E=O B$

$1: A E=03$
$2: 3 E=03$

$4.9 E-03$
$6.0 E-03$

$: 6 E-02$
$1: 1 E-02$

$1: 6 E-03$

9. $8 E-04$

5. $0 E=04$

1. $\mathrm{BE}-04$

5.2E-03 $1.4 E-0$

$2.9 E-04$

$6: 9 \varepsilon-05$

$1.1 \mathrm{E}-03$
$1.6 \mathrm{E}-03$

C.7E-03

$1: 4 E-02$

$1.26 E-04$
$8.65-05$

7: $1 E-05$

$1.1 E-02$
$7.8 E-03$
$1.0 E-03$

$\because 5 E-02$

$1.3 E-01$

$\because: \pi E-03$

$7: 2 E-03$
$7: 9 E-04$

$\therefore 9 E-03$ 2.

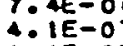

$7: 4 E-06$

$4: 1 E=07$
$3.6 E-07$

$1.6 E-03$
$2.6 E-06$

$6.6 E-07$
$6.3 E-07$

$2,1 E=07$

B: $1 E=07$

$2.3 E-0$

$1.0 E-06 \quad 3: T E-07$

$7.5 E=07$

6. $6 E=0$

$3.3 E-08$
$9 B E-05$

$1: 0 E=04 \quad 9: 0 E-05$

$1.5 E-03$

$7.2 E-03$
$B .0 E-03$

$2 \cdot 0 \mathrm{E}-02$
$2.2 \mathrm{E}-02$
6.02

$2.5 E-06$

$2.2 E-06$
$i: 4 E-O T$

$2.1 E-03$
$1.6 E-02$

$6.4 E-03$
$4.6 E-02$

I. $7 E-03$
KIONEYS

KLONEYS LIVER

1:1E-OY $1: 2 E-07$

OSE-OB $2.4 E-O A \quad 1 . O E-O B$

T.

.7E-05 T:OE-05 3:DE-OS

.3E-OS $3.2 E-05$ 2.AE-0S

$.3 E-0 B$ 2.8E-OB $1.06-O B$

$1.2 E-08 \quad 2.7 E-08 \quad 1.5 E-08$

7.3E-D4 2.TE-03 $6.7 E-04$

9.4E-04 9:JE-04 9.6E-04

$2.7 E-05$ 2:TE-OS $2.7 E-05$

T.9E-06 B.1E-06 7.9E=06

$1.5 E-03 \quad 1.5 E-03 \quad 1.5 E-03$

7:1E-06 7:3E-06 $3: 1 E-06$

$\begin{array}{lll}2.3 E-06 & 2: 9 E-06 & 2.5 E=06 \\ 5.0 E-04 & 5: 0 E-04 & 0.8 E=004\end{array}$

$\begin{array}{lll}0.0 E-04 & 5 . A E-04 \\ \because 7 E-006 & 4.2 E-04\end{array}$

$2.7 E-06 \quad 4.6 E-06 \quad 3.4 E-06$

$1.3 E-03 \quad 1.3 \varepsilon-03 \quad 1.3 \varepsilon-03$

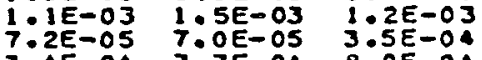

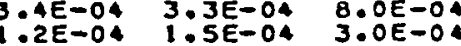

6.0E-05 7:3E-05 6:0E-O5

OAE-05 $1.3 E-04 \quad 1.2 E-04$

$1: 1 E-04$ 1:3E-04 1:2E-O4

$\begin{array}{lll}0.5 E-05 & 1: 4 E-05 & 1: A E-0 S \\ 5: 2 E-02 & 4: 7 E-02 & 4: B E-02\end{array}$

$4.6 E-03$ 4.6E-03 $4.6 E-03$

$8.0 E-03 \quad 7.5 E-03 \quad 7.5 E-03$

$\begin{array}{lll}3: 0 E-07 & 3: 3 E-07 & 3.3 E-02 \\ & 2.4 E-07\end{array}$

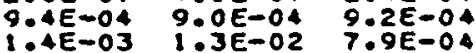

. $1 E-04 \quad 3.0 E-03 \quad 6.1 E-04$

3.6E-04 1.3E-02 2.7E-04

$9.0 E=051: 9 E-03 \quad 905 E-05$

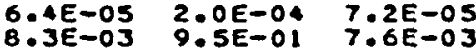

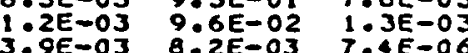

$2.2 E-04 \quad 4.5 E-04 \quad 4: 1 E-07$

$2.3 E-07 \quad \because .9 E-07 \quad \because 00=07$

$\begin{array}{ll}3.6 E-07 & 7: B E-07 \\ 1: 0 E-07 & 7.5 E-08\end{array}$

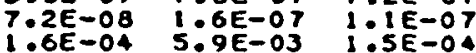

$1.0 E-04$ 6.BE-04 1.3E-04

$2.4 E-06 \quad 1.0 E-01 \quad 2.3 E-06$

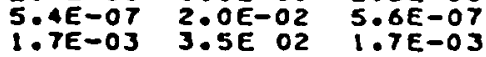


(REMUHICROCURIE)

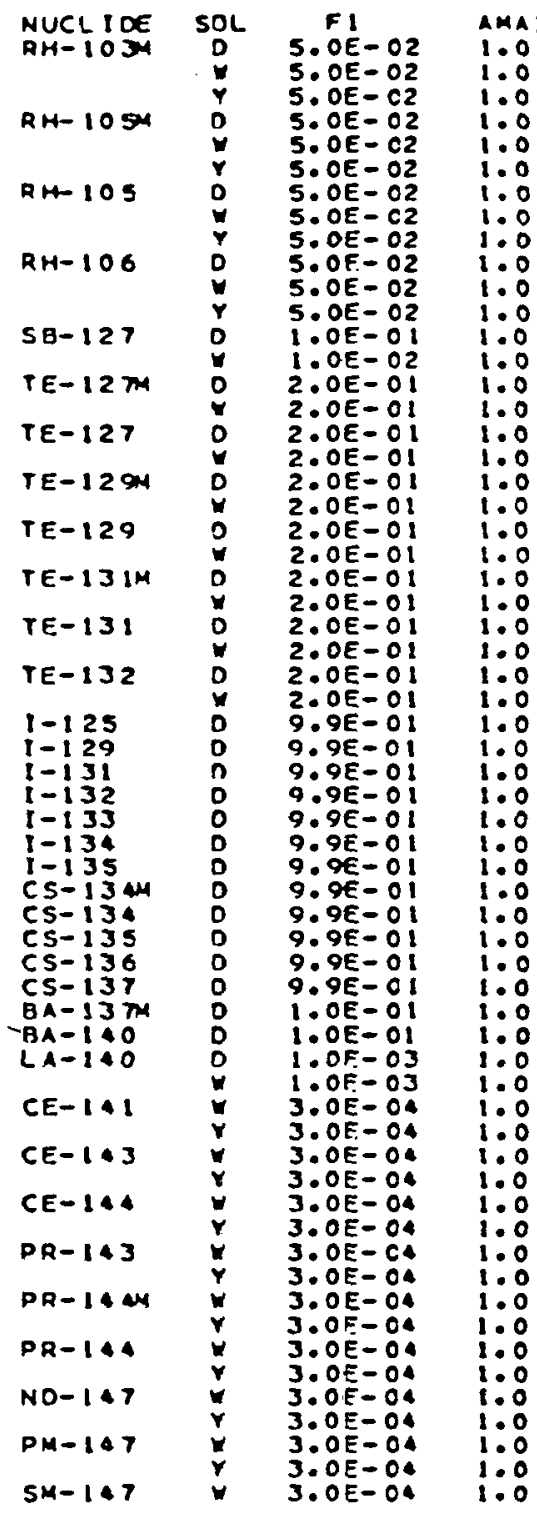

\begin{tabular}{|c|c|c|}
\hline & 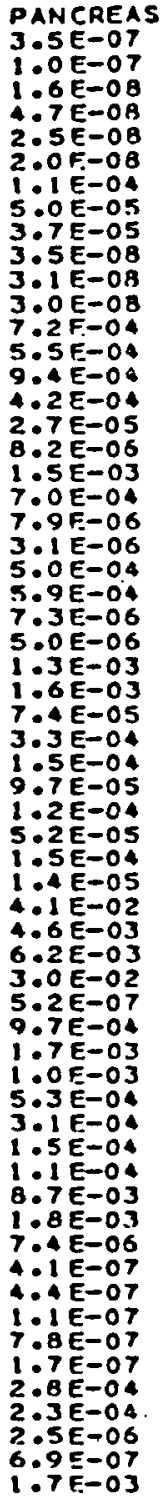 & 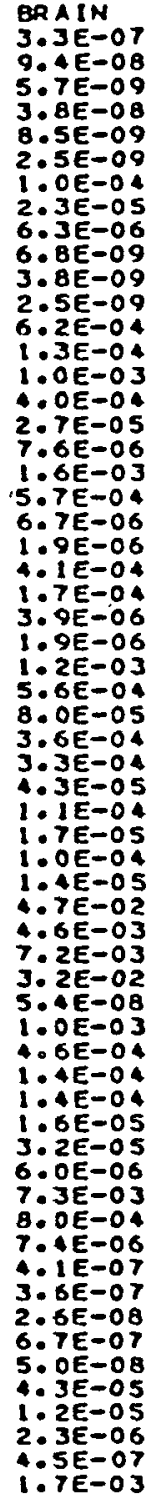 \\
\hline
\end{tabular}

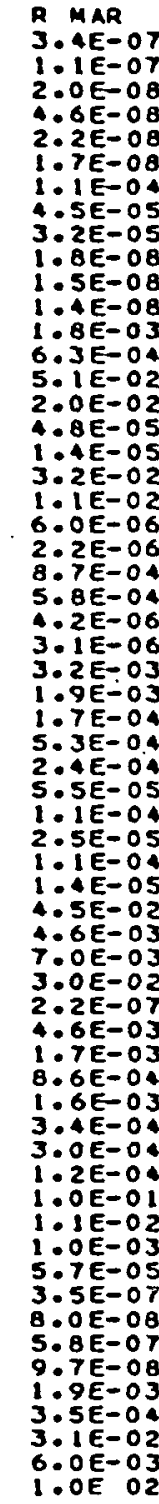

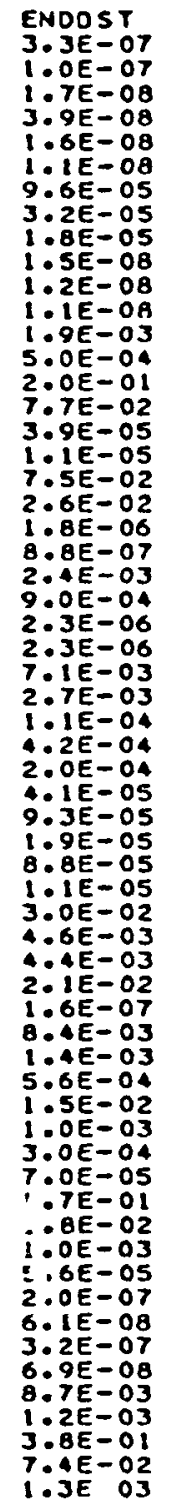

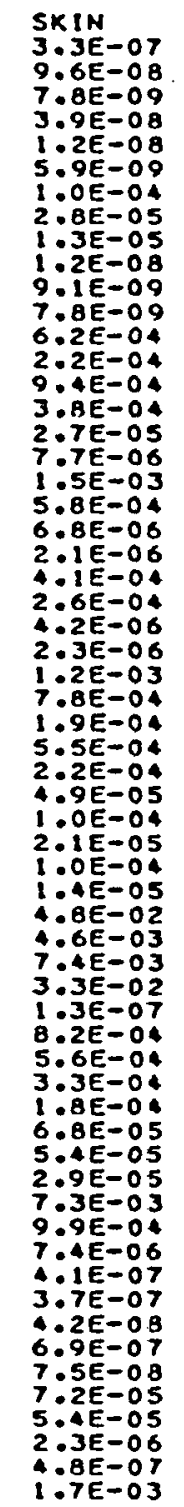

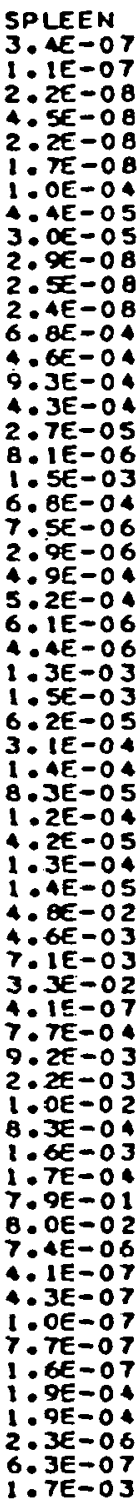

TESTES

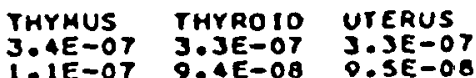
9. $5 E=08$

$3.9 E=08$
$0 B=-09$ $0.0 E-09$
$1.0 E-09$ $1: 1 E-04$
$2:>E E-05$
$1.1 E$ $4.7 E=09$ $1.6 E-09$
$30 E-10$ 3. $9 E-10$ 1. $7 E=04$ $3.6 E-04$ 2.:6E-06 $1 \cdot 5 E-03$
$5.3 E-04$ 6. $6 \mathrm{E}-06$ 3. $7 E-0$ $1.8 E-04$ 1. $3 E-06$ $1.1 E=03$
$5.9 E=04$ 7.0E-05 3.2E- 94 $3: 4 E-05$ : 4 AE-OS 7: $7 E=05$ $41 E-02$ $4.6 E-03$
$6.3 E-03$ $3.0 E=02$ $2.4 E=08$ $4.6 E-04$ 2.2E- 104 $2.1 E=05$
$4: 4 E-05$ 2. $2 E=05$ $7.4 E-04$ ?
:
:E $=06$ 3. SE-07 $2.1 E-08$
$6.6 E-07$ 3.9E-08 2.6E-05 $2.2 E-06$
$4: 4 E-07$ i. $7 E-03$

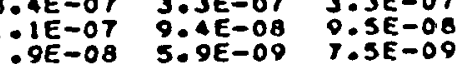
$\begin{array}{lll}30-08 & 4.0 E-0 B & 1.0 E-O B\end{array}$ 1.JE-08 $1.7 E-08$ :OE-O 5.OE-05 $2: 9 E-05 \quad \because T E-05$

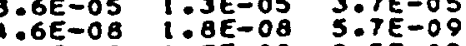
$\begin{array}{rl}4 E-08 \\ 1.5 E-08 & 2.5 E-09\end{array}$ O. $5.9 E-042.6 E-04$ O.SE-OA .5E-04 3.8E-04 3.7E-04 $2.7 E-05$ 2.7E-0S 2.7E-0S $8.2 E-06 \quad 7.7 E-06 \quad 7.9 E-06$ $1.5 E-03 \quad 1.5 E-03 \quad 1.5 E-03$ 7.AE-O6 $609 E-06$ $7.7 E-04$ 3. $4 E-06$ 2.3E-06 2.0E-08 $7.1 E-04$ S.BE-02 $3.7 E-04$ 7.4E-06 2:1E-03 $3: 9 E-06$ $7.5 E-06 \quad 1.7 E-03 \quad 1.4 E-06$ O :OE-OA B.IE-OI $600-05$ .2E-OA $5.8 E$ OO $3.3 E-0.4$

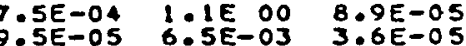
$2.1 E-042.7 E-01$ 7.4E-05

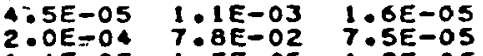
$1: 4 E-05$ 1:SE-05 1:2E-05 $42 E-02$ 5.4E-02 $4.0 E-02$ $4.6 E-03 \quad 4.6 E-03 \quad 4.6 E-03$ $6.6 E-03 \quad 9.2 E-03 \quad 6.0 E-03$ $\begin{array}{lll}3.0 E-02 & 3.5 E-02 & 2.9 E-02 \\ 6.1 E-07 & 2.2 E-07 & 4.3 E-0 B\end{array}$ 7.9E-04 B.6E-O4 $9: 1 E-04$ $\begin{array}{lll}0 B E-04 & 8.0 E-04\end{array}$ $4 J E-04 \quad 1.9 E-04 \quad 2: 1 E-04$ $4.6 E-049.7 E-05$ 9.6E-05 OAE-O4 S.SE-05 $1.2 E-04$

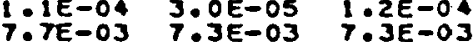
2.4E-03 $\quad 1.1 E-03$ B.JE-04 7.4E-06 7:4E-06 $7: 4 E=06$ $\because-1 E-07 \quad \because-1 E-07$ A.IE-0T $.9 E-07 \quad 3.8 E-07 \quad 3.6 E-07$
$0.6 E-07$ 1.6E-OT $\because .8 E-0 B \quad 2.6 E-0 B$ $2.5 E-07 \quad 9.6 E-08$
$0.9 E-0 A$ B.3E-05 $1.3 E-04$ $\begin{array}{lll}2.8 E-04 & 7.2 E-05 & 1.2 E-04 \\ 2 E-06 & 2.2 E-06 & 2.2 E-06\end{array}$ $7: 9 E-07$
$1: T E-03$
I:OE-07 
A ALPHACOE TO
(REMIMICROCURIE)

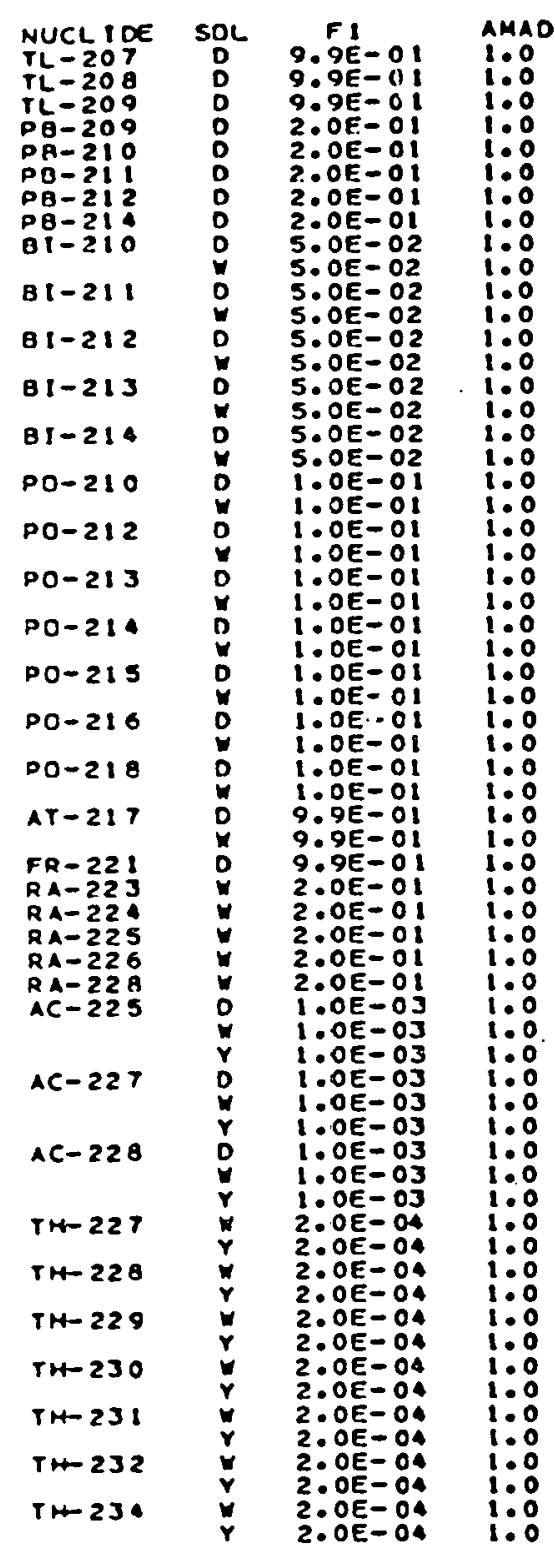

EFFECT. LUNGS $\begin{array}{ll}0.5 E-06 & 6.5 E-05 \\ 9.03 E-06 & 6.6 E-05\end{array}$ $\begin{aligned} & 7.8 E-06 \\ & 8.4 F-05\end{aligned} 4.7 E-05$ $\begin{array}{lll}4 E-01 & 1.2 E & 00 \\ 0.6 E-03 & 7.5 E-02\end{array}$ 9.TE-01 $7: 3 \mathrm{E}=0$ $8: 9 \in-03605$ $\begin{array}{ll}2.0 E-01 & 1.6 E-00 \\ 1.0 E-03 & 8.6 \mathrm{E}-03\end{array}$ $1.1 E-03 \quad 0.2 E-0$ $\begin{array}{ll}2.5 E-02 & 1.5 E E \\ 3.0 E-02 & 2.7 E-0\end{array}$ $9.5 E-04 \quad 5.9 E-03$ $\begin{array}{ll}.3 E-03 & 9.9 E-0 J \\ 91 E-03 & 6.6 E-02\end{array}$ 1.2E-02 $9.4 E-02$ $3.06-12$ 3.0E-11 3.6E-12 3.0E-11 $4.7 E-11$ 3.9E-10 $\begin{array}{ll}.7 E-11 & 3.9 E-10 \\ 7.7 E-0 S & 1.4 \mathrm{E}-00\end{array}$ $\begin{array}{ll}1: T E-09 & 1.4 E-08 \\ 2.7 E-08 & 2.2 E-07\end{array}$ $3.2 E-0 \& 2.6 E-0$ $2.3 E-06 \quad 1.9 E-05$ $\begin{array}{ll}3.1 E-03 & 2.5 E-02 \\ 3 & 1 E-07 \\ 3 & 2.6 E-06\end{array}$ $3.2 E-072.7 E-06$ G.SE-0J $3.7 E-02$ 3.GE 00 2.8E OI 8.9E 우 0 6.1E OAE $\therefore 0$ M.3E O 6.IE DO S:OE OI 6.7E :3 4.5E-O $\begin{array}{lll}1.3 E & 03 & 5.6 E \\ 2.9 E-01 & 2.5 E\end{array}$ $2.9 E-0$ B. $8 E-02$ I $3 E-0$ 1 SE O1 9:0E O1 $1.6 E$ O1 1.36 $3.4 E$ O2 $2.6 E$ O3 1.6E O3 1.35 . 3.IE O2 6.0E O $8.5 E-043.9 F-0$ $8.5 E-04 \quad 4: 4 E-03$ $1.1 E \quad 03$ $\begin{array}{ll}2.9 E-02 & 1.8 E-0 \\ 3.4 E-02 & 2.4 E-01\end{array}$

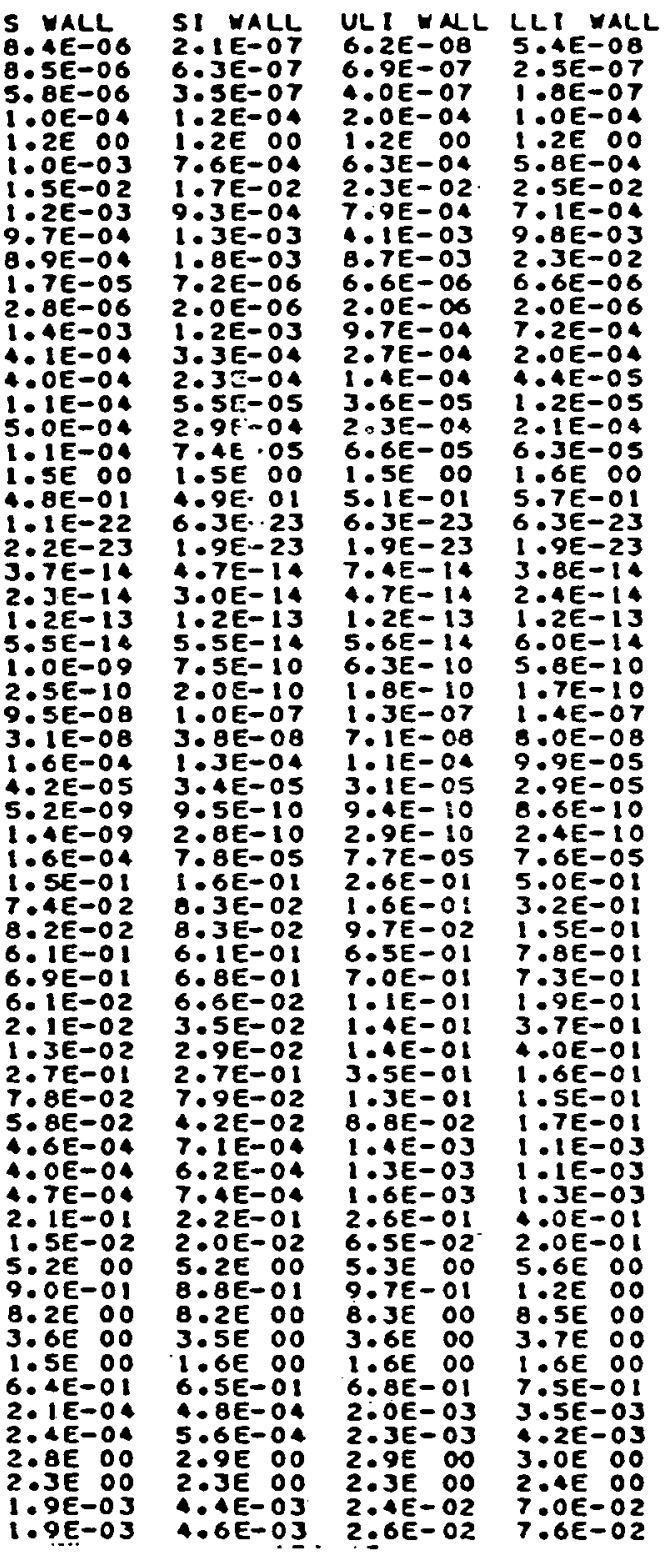

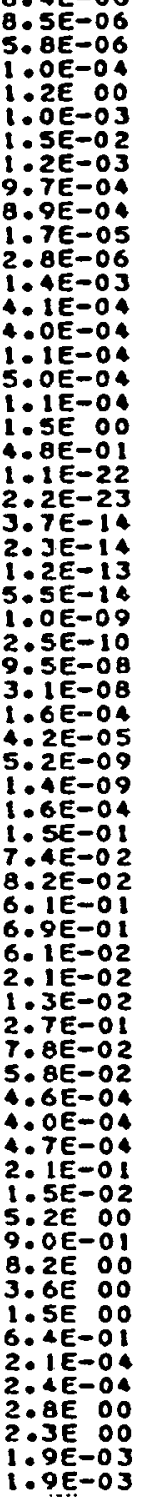

$A D R E N A L$
$1.1 E-0$ i: 1 eE-0. $6.4 E=06$ $\begin{array}{ll}1.2 F & 00 \\ 5 . A E-O A & \end{array}$ $5 . A E-O 4$
$1: A E-02$ $7: 1 E-04$ 7:SE $2: 04$ 6. $T E-06$ 2. $0 E-06$ $2: 0 E-04$ $5.2 E-05$ $3.0 E-05$ $2.2 E-04$
$6.9 E-05$ 6. $9 E-05$ $1.5 E$ OO 1.SE : D $1.9 E-23$ O.AE-23 $2 . \pi E-15 \quad 2.7 E-15$ $1: 22 E-13 \quad 1: 2 E-13$ $\begin{array}{ll}5.5 E-14 & 5.5 E-14 \\ 5.8 E-10 & 5 . B E-10\end{array}$ 1. $T E-10$ 1. $T E-10$ $\begin{array}{ll}9.3 E-08 & 9.2 E=08 \\ 2.3 E-08 & 2.3 E-08\end{array}$ 9. $2 E=05$ S. $9 E-05$ $\begin{array}{ll}3.0 E-05 & 2.9 E-05 \\ 1.1 E-09 & 9.4 E-10\end{array}$ $1: 1 E-10 \quad 2.6 E-10$ $7: 9 E-05 \quad 7: 7 E-05$ G.AE-OL $6.0 E-02$ 8.2E-02 8. $1 E-02$ $\begin{array}{lll}6: 1 E-01 & 6.1 E-01 \\ 6 & 9 E-01 & 6.9 E-01\end{array}$ $6.2 E-02$ 5.6E-02 2. $6 E-0 J$ I. $A E-03$ $\begin{array}{lll}0: B E-01 & 8 . \Delta E-02 \\ 1.0 E-01 & 2: 9 E-02 \\ 0.0 E & & \end{array}$ $1.0 E-01 \quad 1.6 E-02$ 1:2E-04 $: 2 E-05$ 2: $1 E-012$ 2. IE-OI $1.2 E-02 \quad 1.1 E-02$ 5.2E $O O$ 5. $2 E D O$ 9.OE OO B. JE OD 3.OE OD 3.SE DO 6. 1. ZE-O5 . TE-06 2:AE OD $2:$ : $4: A E-04$
$T E-05$

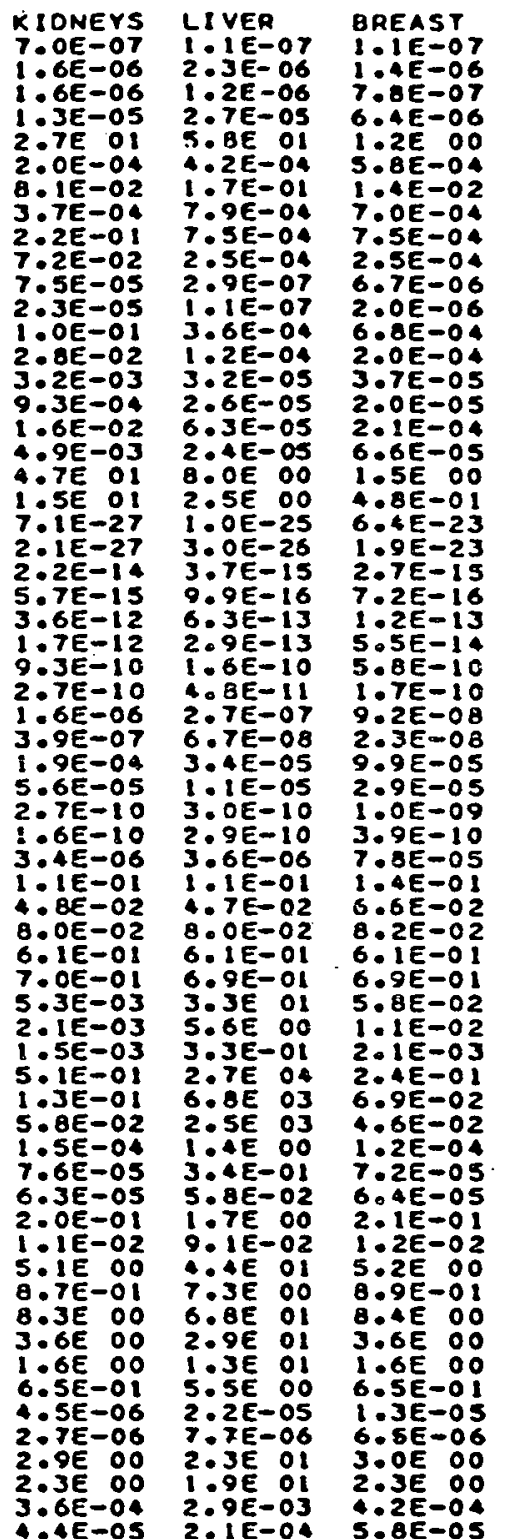

AE-05 2:IE-OA 
OVARIES PANCREAS BRAIN

$\begin{array}{lll}0.1 E-07 & 1.1 E-07 \\ 3.0 E-07 & 2.0 B-06 & 1.1 E-07\end{array}$ $2.8 E-07 \quad 1.5 E-06$

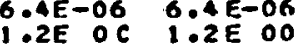
5.AE-O4 5.8E-0. $\begin{array}{ll}1.5 E-02 & 1.4 \mathrm{E}-02 \\ 7.0 \mathrm{O}-04 & 7.1 \mathrm{EEOA}\end{array}$ T.SE-OA T.5E-O4 $6.7 E-06 \quad 6.7 E-06$ $\begin{aligned} & 2.0 E-06 \\ & 0.0 E-04\end{aligned} 6: 0 E-06$ $6 . A E-04$
$1.9 E-04$
$3.1 E-O A$ $\begin{array}{rl}1.9 E-04 & 2.1 E-04 \\ 3: T E-05 & 6.2 E-05 \\ 0.05 & 35 E-05\end{array}$ $1: 1 E-05 \quad 3.5 E-05$ $6.3 E-05$ T:OE-OS

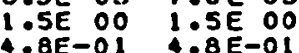
$\begin{array}{rl}4 . B E-01 & 4.8 E-01 \\ 6.4 E-23 & 6.4 E-23\end{array}$ 1.9E-2J $1.9 E-23$ $2.7 \mathrm{E}-15 \quad 2.7 \mathrm{E}-15$ $22 E-16$
$1: 2 E-13$ $5.5 E-14$
$5.5 E-14$
$5.8 E-10$
$5.8 E-10$ $\begin{array}{rl}5 . \Delta E-10 & 5.8 E-10 \\ 1.7 E-10 & 1.7 E-110\end{array}$ $2.4 E-08 \quad 2.3 E-0 B$ $9: 9 E-05$ I.OE-OA $2.9 E-05 \quad 3: 0 E-05$ $2.6 E-10 \quad 5.5 E-10$ $7.7 E-05$
$1.0 E-01$
$1.0 E-05$ $6: 7 E-02 \quad 6: 7 E-02$ $\begin{array}{ll}6: 1 E-01 & 6: 1 E-01 \\ 6: 9 E-01 & 6: 9 E-01\end{array}$ 5.8E-02 6.1E-0? $1.3 E-02 \quad 1.2 E-02$ $1.5 E$ O 5 5.Y 3.TE O2 $1: 5 E=01$ $1.6 E-04 \quad 2.1 E-04$ $1: 0 E-04$
$1.0 E-04$
$1.3 E-04$ $2.1 E-01 \quad 2=1 E-01$

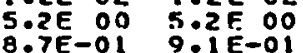
8.TE-01 9:1E-O1 8.JE DO A.4E DO G.5E-OI 6:SE-OI $2.4 E-O S \quad 1.3 E-05$

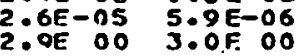
$2.3 E-00$
$4.3 E-04$
$1: 3 E-O D$ $4.3 E-04$
$7.3 E-n 5$ 政 $2.5 \mathrm{E}-04$ 6.7E-06 6 TE 04 $1.9 E-04$
$2.0 E-05$ $9=9 E-06$ 6: $3 E-05$ $1: 5 E 00$ $4.8 E-01$
$6.4 E-23$ $1 \cdot 9 E-23$
$2.9 E-15$ $7: 2 E-16$ $5.5 E-14$ $1.8 E-10$
$1.7 E-10$ $2.25-08$
$2.3 E-008$ $9.9 E-05$ $2.9 E-05$
$9,4 E-10$ $2.8 E-10$
$7.7 E-05$ $7: T E=05$
$1: A E-01$ $6.6 E-02$
$8: 1 E-02$
$6.1 E-02$ 6. $1 E-01$ $6.9 E-01$
$5.7 E-02$ 9.7E-. 2. $3 E-01$ $6.5 E-02$
$3.0 E-02$ $9: 0 E-05$
$2: 9 E-05$ 2.9E-05 2:1 $1 E-01$ 5. $2 E .02$ $\begin{array}{ll}8.6 E & 00 \\ 3.6 E & 00\end{array}$ $\begin{array}{ll}3.6 E & 00 \\ 1.68 & 00 \\ 6 & \end{array}$ $6.5 \mathrm{E}-01$
$7.9 \mathrm{E}-06$ 6. उE-0?

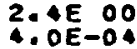
2.: $0 E-0.04$ p MAR $\begin{array}{lll}0.6 E-07 & E N O J S T \\ 1.2 E-07 & 0\end{array}$ 2.2E-05 2.2E-04

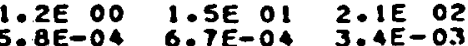
$1.4 E-02 \quad 1.2 E-01$ 1. $4 E$ DO $3: 1 E-03$

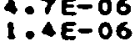
7.4E-04 $2,1=05$ 1.91-04 I. 1 E 00 $3.4 E-01$
$43 E-23$ 2. $3 E-15$ $6: A E-16$ $8.3 E-14$
$4.2 E-10$ $1.2 E-10$ $2.6 E-08$ $2.1 E-05$ $3.3 \mathrm{~B}-10$ $5.4 E-05$
$8,9 E-01$

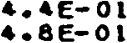
$2.5 E$ 9.9E 00 $\begin{array}{ll}1: 6 E & 00 \\ 9.9 E-02 & 02\end{array}$ $9,4 E$ O3 $85 E-O 2$ \begin{aligned}$\because \because 2 E-0 \\$\hdashline$O E-O I\end{aligned}$ : $8 \mathrm{BE-02}$ $\because 1 E-00$
$: 9 E-01$

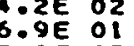
3 ZEE : $\begin{array}{ll}6.2 E \\ 2.5 E & 02 \\ 3 & 02\end{array}$ $3.0 E-04$ $1: 5 E .03$ $1.5 E-02$
$B .9 E-04$
SKIN $1: 1 E-07$
$B: 2 E-07$
B. $5.0 E=07$ $1.2 E 00$ 1:1 $3 E-03$

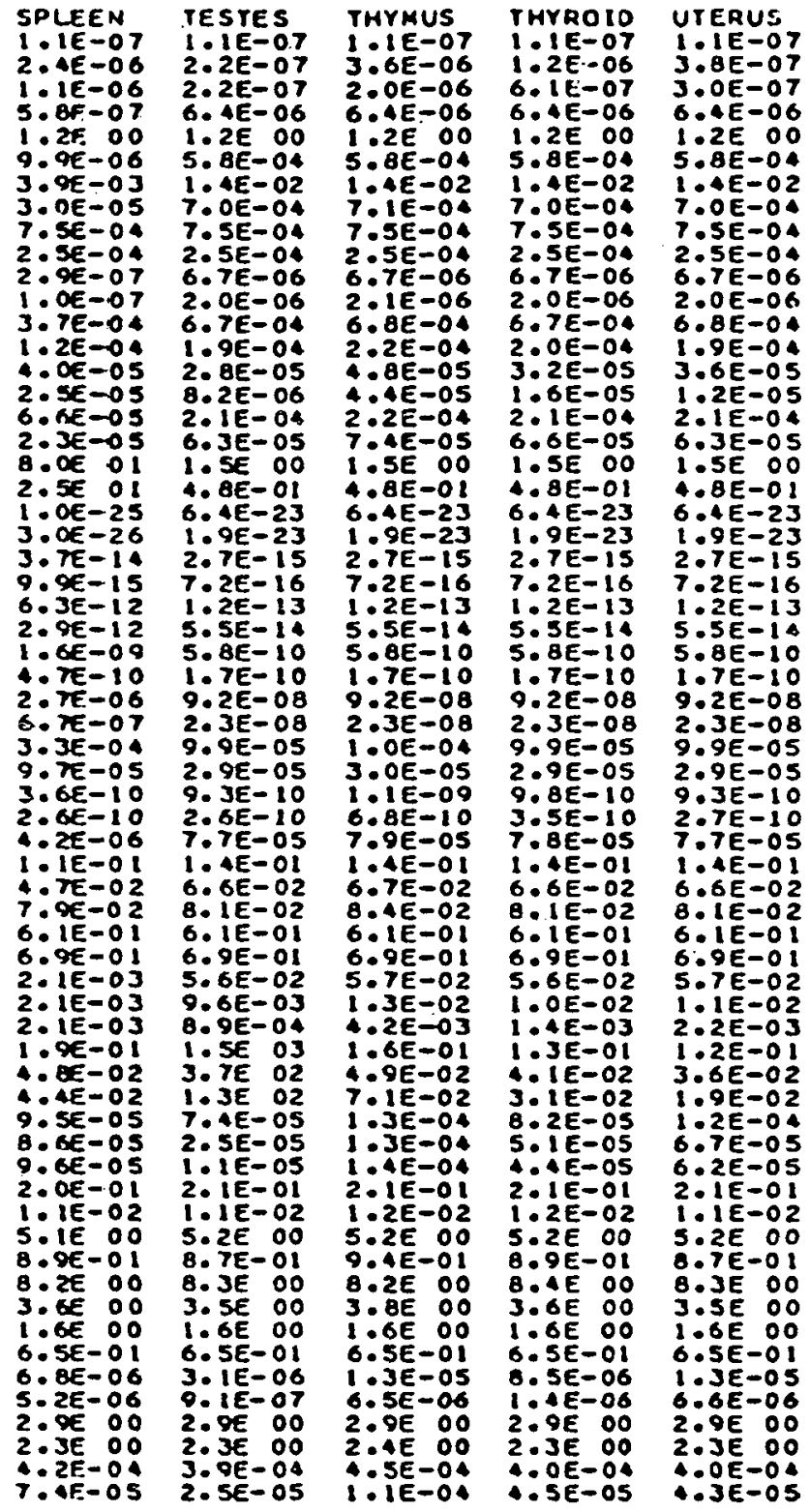


INMALATION DOSES AT SO TEARS (REMPHA ICPOCURIE)

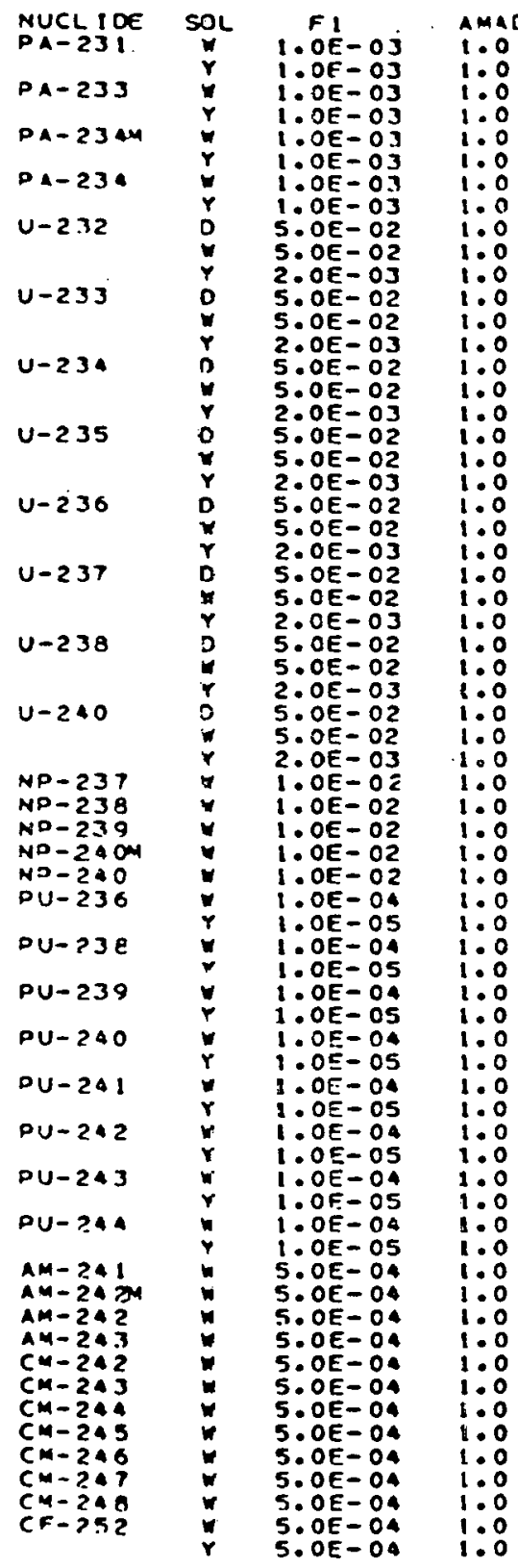

EFFECT LUNGS

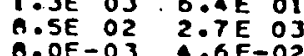
$9.2 E-03 \quad 6: 4 E-0$ $3 . A E-06 \quad 3.1 \leq-0$ 8. $6 E-045$ 5 51 l.2E Ol $1: 5 E$ OO 1.SE Ol $9.3 E$ OL 2. TE DO 1.2E OO I.JE O2 1.1E OJ 2.TE OO $1: 2 E$ OO :JE O2 I:DE OI 2.SE DO T.1E 000 I.ZE O2 $1.0 \mathrm{E}$ O $\begin{aligned} & 2.5 E \\ & 7.56\end{aligned} 00 \quad 5: T E$ OI

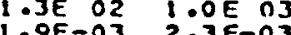
$3.2 E-03 \quad 1: 7 E-0$ 3.AE-O3 $1: 9$ E. T:OE DO $5.3 E$ OI

$.1 E-03 \quad 4.5 E-03$ $1.8 E-03 \quad 1: 1 E-02$ $5.0 E$ OZ $6.0 \mathrm{O}$ OI $2 . A E-03$ I:IE-O2 $1.3 E-0 \&$ O. $.8 E-O$ $1.7 E$ O? $6.9 E$ O 1

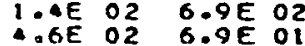
3 .IE O2 1.2E O S.IE O2 $0.5 E$ OI $5.1 E$ O2 6.5E Ot 1 . OF O1 2.7 F-O $\begin{array}{llll}5 . \pi E & 0 O & 1.2 E & 01 \\ 1.9 E & 02 & 6: 1 E & \text { OI }\end{array}$ $3.2 E$ OS $2.0 E-0 A \quad 1.2 E-0$ $\triangle B E$ O2 6.1F O 3.2E O2 1 I.IE OJ

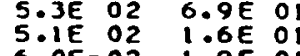
6. $0 E-02$ $11.96-0$ $1.0 E$ OI $5.0 E$ OD

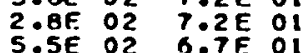
$\begin{array}{llll}5.5 E & 02 & 6.7 E & 01 \\ 5.4 E & 02 & 6.0 E & \text { OI }\end{array}$ ?.OF O. $2.5 \mathrm{~L}$ O?

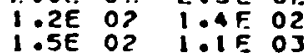

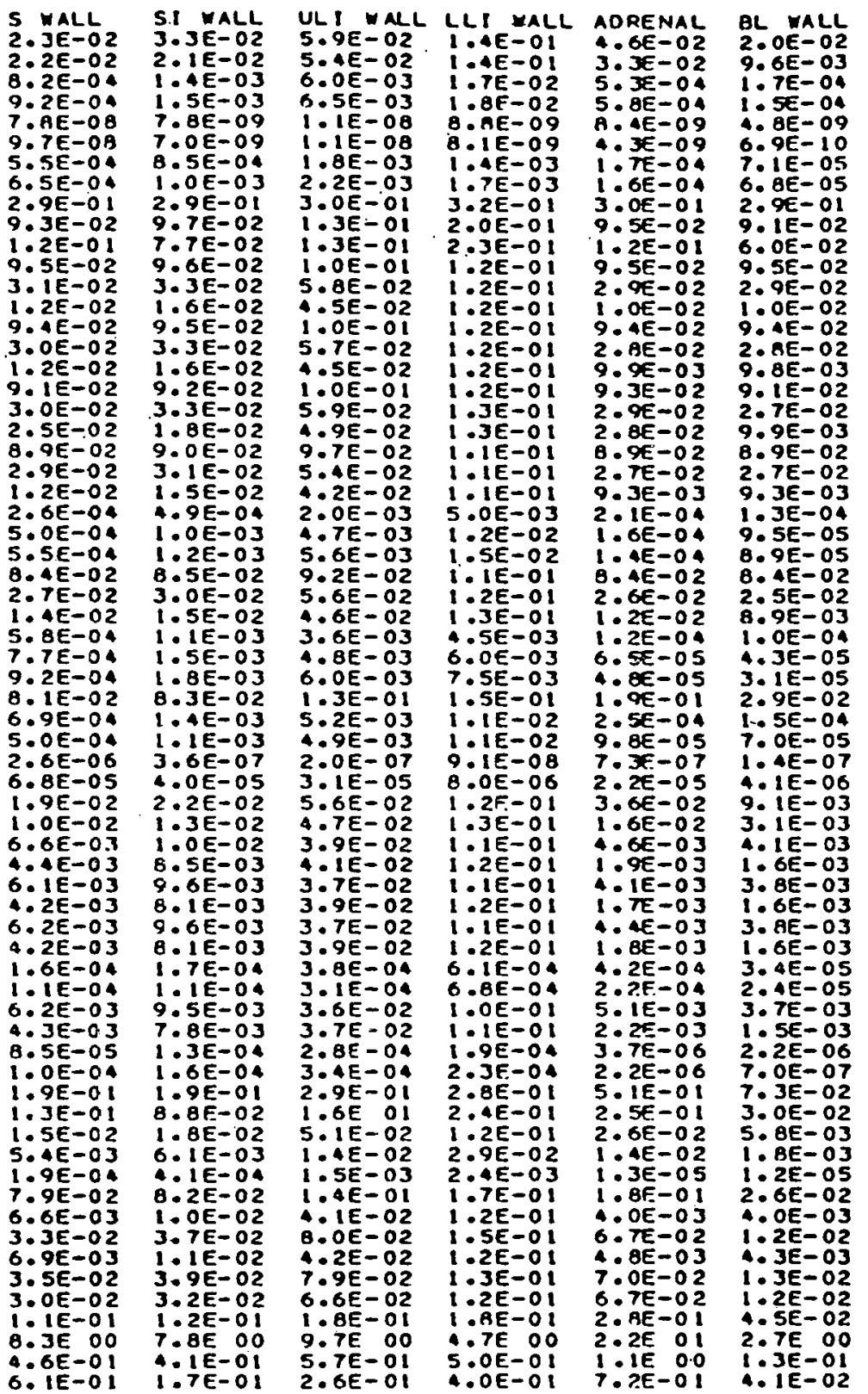

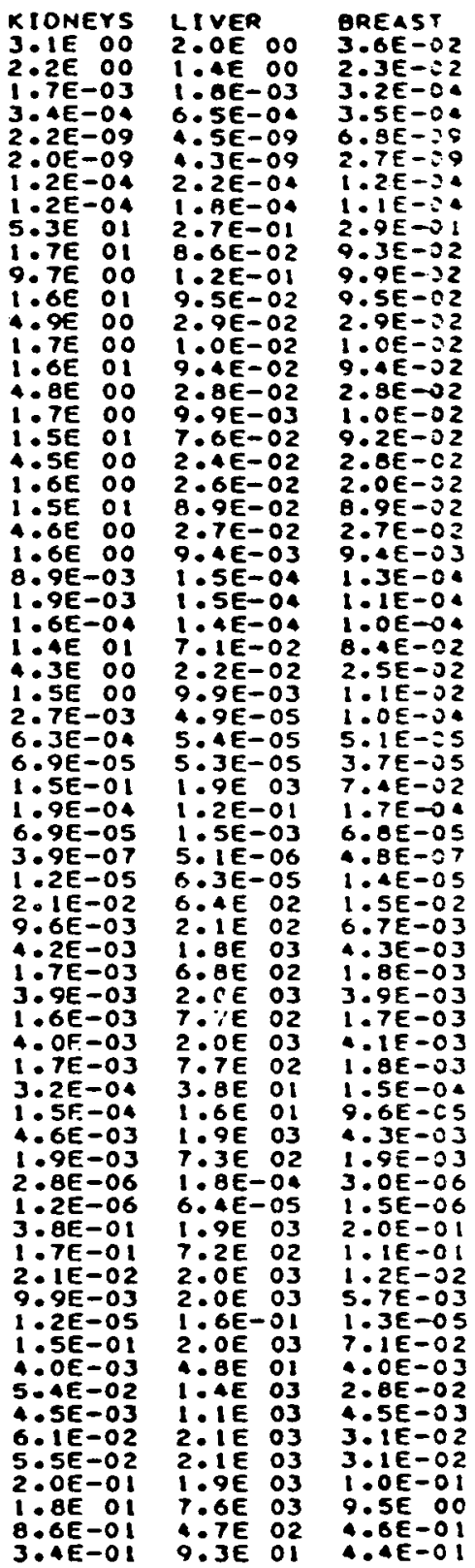
: 


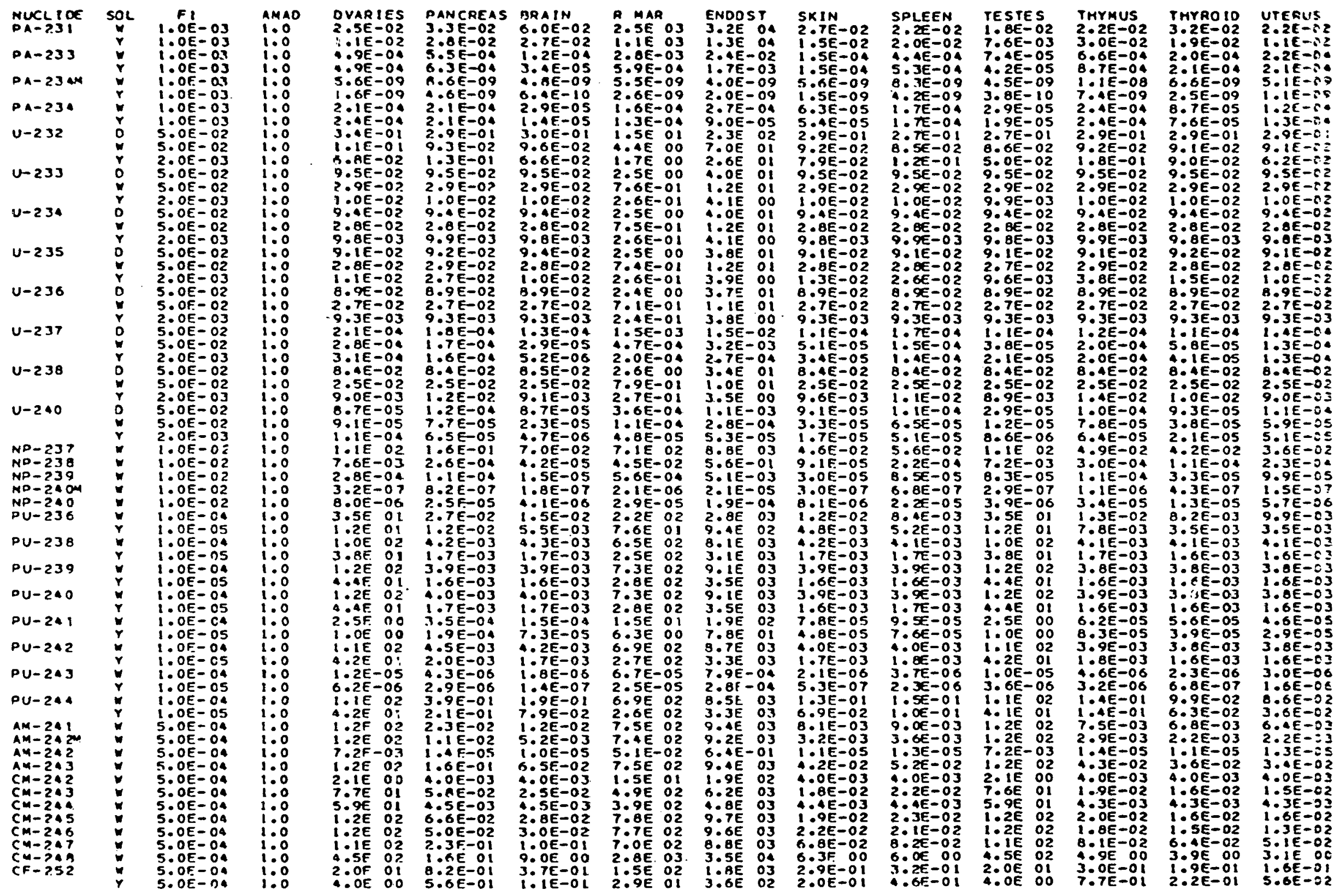


(REMAMICROCURIE)

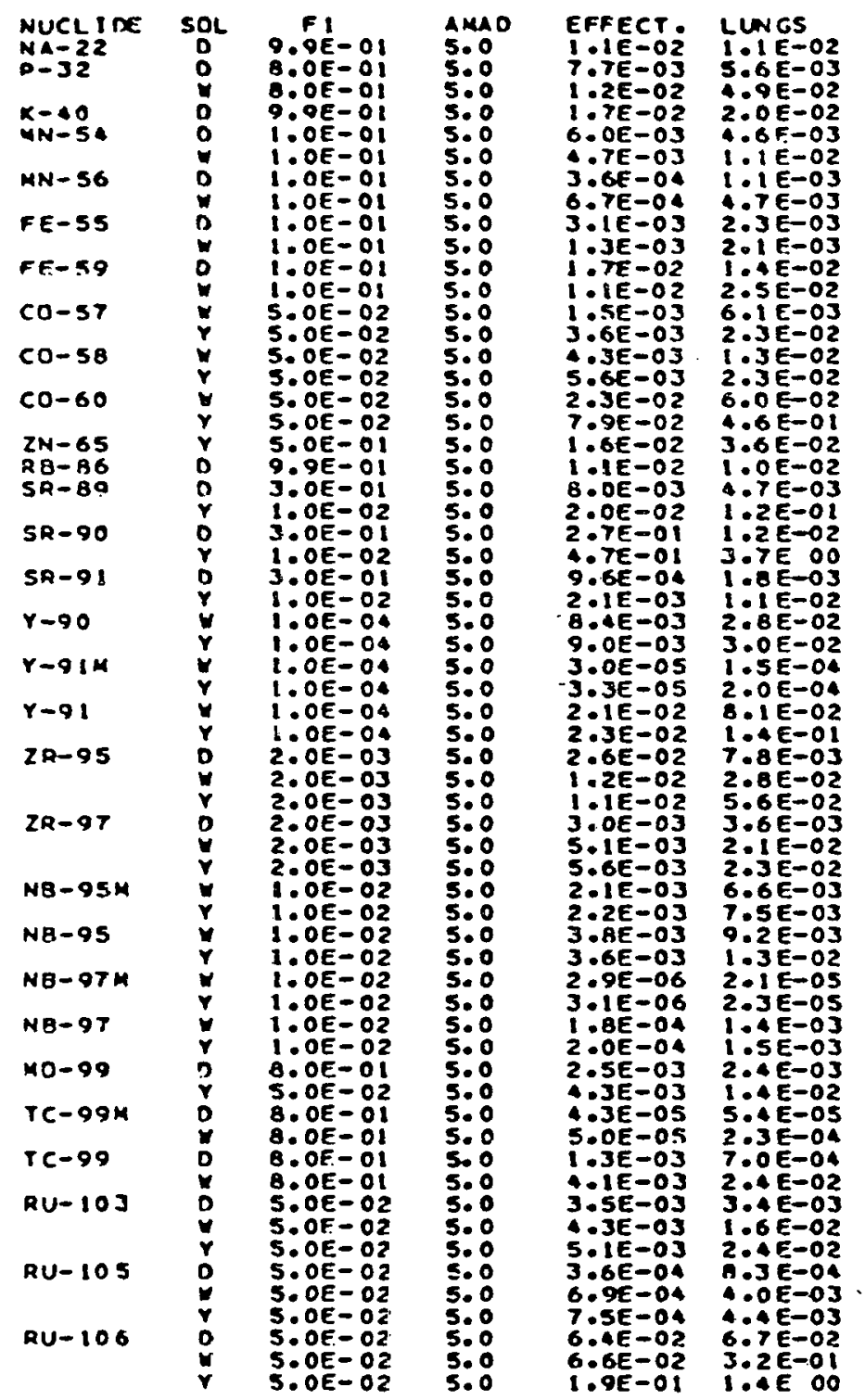

\begin{tabular}{|c|c|c|c|c|c|}
\hline 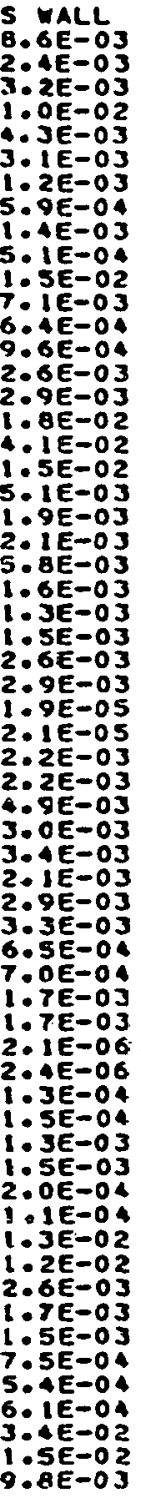 & 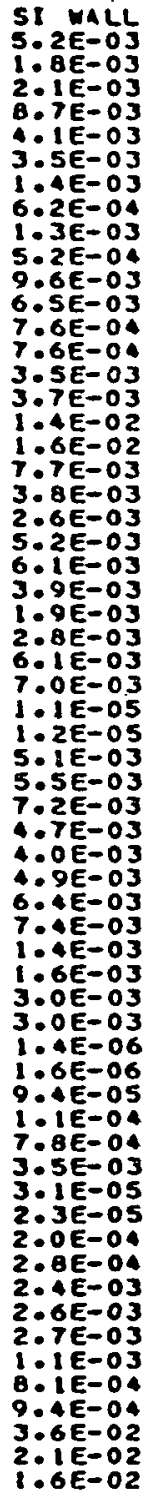 & 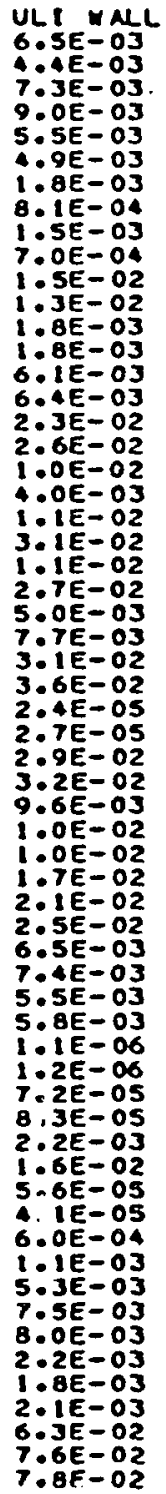 & 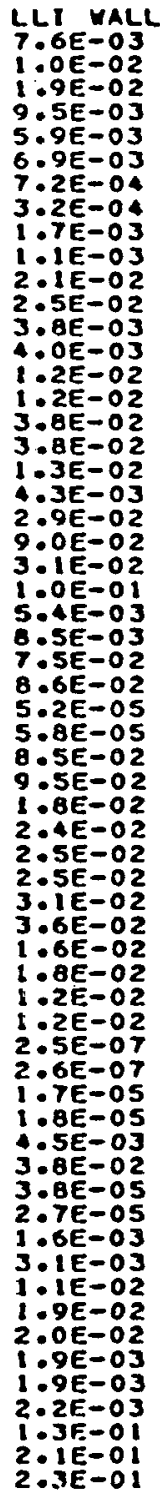 & 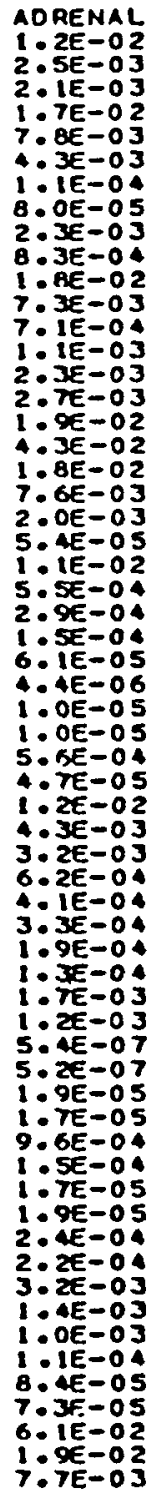 & 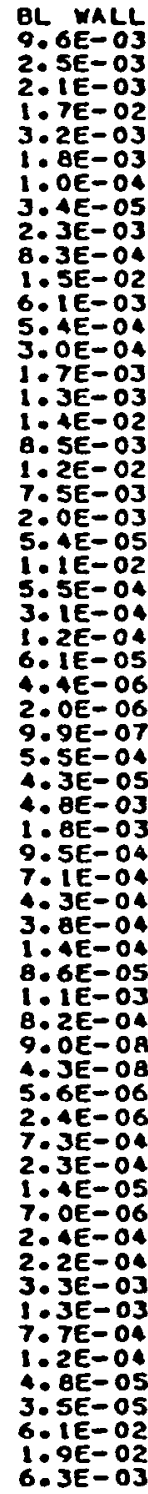 \\
\hline
\end{tabular}

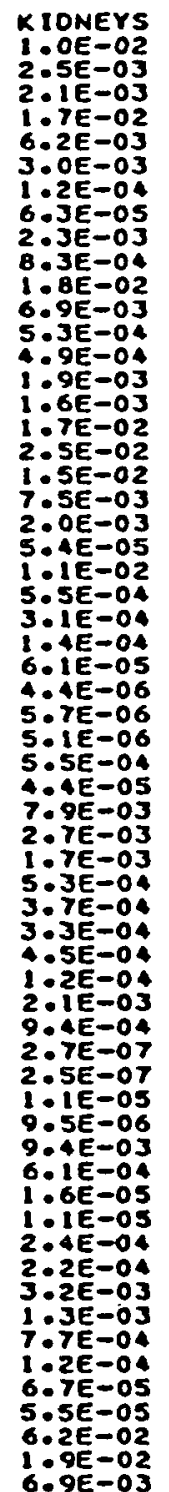

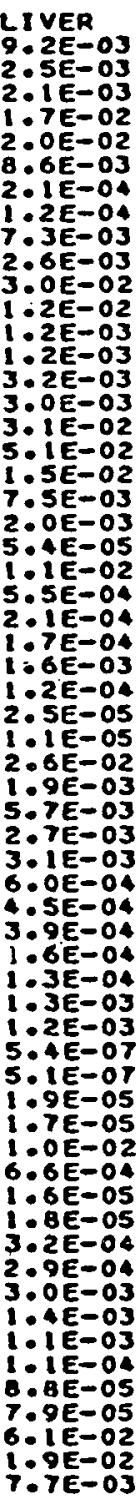

BREAST

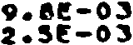

$2.18-0.03$

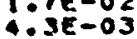

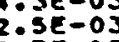

$2: 3 E-03$

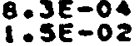

$6.1 E-03$

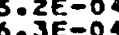

$1.0 E-03$

$1.0 E-0.03$
$1.05-0.03$

$1.5 E-02$
$2.8 E-0.25$

$1.4 E-02$

$7.5 E-03$
$2.0 E-0.03$

$5: A E-05$

$5,1 E=02$
$1: 5 \in-0.02$

2:BE $\rightarrow 04$

$1.2 E=04$

6.1
$4.4 E=06$

$5.7 E-06$

S.SE-04

$4.4 E-05$
$3.2 E-0.03$

2. $9 E-03$

$2.0 E-03$

6. $1 E=04$

$3.0 E=04$
$3.0 E=04$

1.SE-OA

9.SE-0S

$9.1 E-04$

$2.9 E-07$

$1: 3 E-05$

I.0E-05

$1.5 E=04$
$1.4 E-04$

:AE-05

$2.4 E-04$

$2.2 E-04$

$3.0 E-03$

$1.2 E-03$
$0.2 E-0.04$

$1.0 \mathrm{E}-04$

列

$5: 1 E=05$
$6: 1 E=02$

$1.0 E-02$ 
INHALATION DOSES AT 50 VEARS

(REMIMICROCURIE)

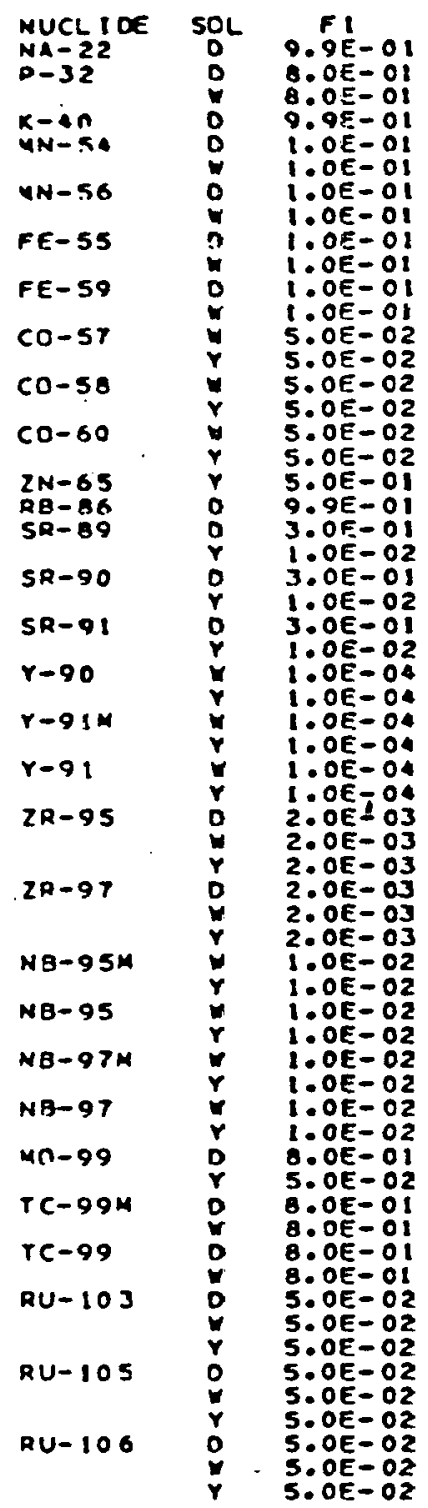

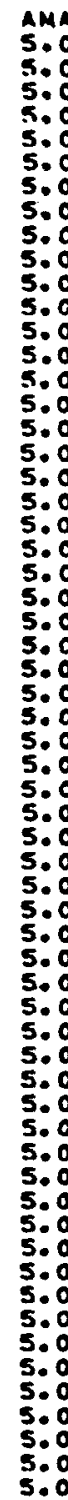

OVARIES PANCREAS GRAIN

R MAR

ENOJST

SKIN

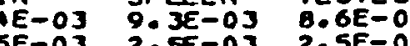

THYMUS

THYROIO YTERUS

$\begin{array}{lllll}2.5 E-03 & 2.5 E-0 J & 2.5 E-03 & 3.2 E-02 & 3.1 E-02\end{array}$

5.0 1.7E-02 1.TE-02

i. $7 E-02$

1.7E-02 $2.6 E-02 \quad 2.1 E-03 \quad 2.1 E-03$

2.1E-03

$1.7 E-02 \quad 1.7 E-02 \quad 1.7 E-02$

$\begin{array}{ll}0.0 E-03 & 2.6 E-03 \\ 2.0 E-03 & 1.1 E-03\end{array}$

$3.5 E-0 J$ J.TE-0J 1.7E-OJ

3.TE-OJ

$1.0 E-02$

$1.8 \mathrm{E}-03$

$7.3 E-05$

$1.1 E-02$

$2 \cdot 6 E-05$

$7 \cdot 3 E-05$
$2.3 E-03$

$9.6 E-05$

$1.0 E-05$

$9.4 E-05$

2. $1 E-05$

$\begin{array}{ll}8.1 E-0 . & 8.5 E-04 \\ 1.3 E-02 & 1.6 E-02\end{array}$

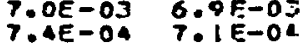

$1.3 E=04$
$1 . A E-02$

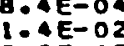

2.3E-03 1.2E-D

$3.6 E-0$
$3.6 E-03$

$8.2 E-04$
$1.3 E-02$

$\begin{array}{lll}1.4 E-02 & 3.6 E-02 & 1.3 E-02\end{array}$

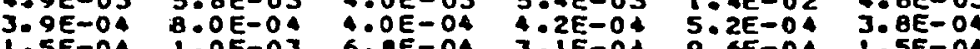

$1.5 E-0$

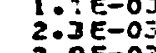

$3.5 E-03$ 2.0E-0.

$\begin{array}{ll}1.6 E-02 & 1.0 E-02 \\ 1.4 E-02 & .6 E-02\end{array}$

$\begin{array}{ll}1.1 E-02 & 1.3 E-02 \\ 7.4 E-03 & 7.4 E-O J\end{array}$

$\begin{array}{rl}7.4 E-03 & 7.4 E=03 \\ 2.0 E-03 & 2.0 E-03\end{array}$

$3.05-031.5 E-0$

S. $3 E-04$

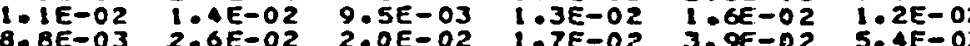

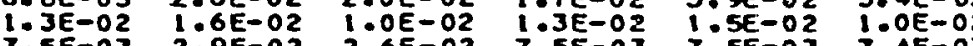

$\begin{array}{llllll}7.5 E-03 & 2.9 E-02 & 2.6 E-02 & 7.5 E-03 & 7.5 E-03 & 7.4 E-03 \\ 2.0 E-03 & 2.6 E-02 & 3.9 E-02 & 2.0 E-03 & 2.0 E-03 & 2.0 E-03\end{array}$

$\begin{array}{ll}5: 4 E-05 & 5: 4 E-05 \\ 1.1 E-02 & 1.1 E-02\end{array}$

S:AE-OS $5: A E-O S$

O.9.0.

$3.0 E-03$

$5: 4 E-05$

5:SE-04 $5: 5 E-02$

5.5E-04

$6.8 E-02$

$1,5 E-01$ 5.5E-0 $501 E-0$

$5.5 E-04$
$2.4 E-0.04$

$2.2 E-02$

$1.6 E-05$

$1.6 E-104$

$9.1 E-05$ 5.SE-OS

3 . OE $=04$

$3.6 E-05$

$\begin{array}{lll}6.1 E-05 & 6: 1 E-05 & 6: 1 E-05 \\ 4.4 E-06 & 4.4 E-06 & 4.4 E-06\end{array}$

$1.0 E-05$

$1.2 E-06$

$1: 0 E-06$

$2.5 E-06$ 1.JEE-0S $8.9 E-07$

2.2E-05

$2.0 E-05$

$\begin{array}{lll}6.6 E-06 & 5.2 E-06 & 2.9 E-06 \\ 2.06-02 & 1.0 E-05\end{array}$

$1: 9 E-03$

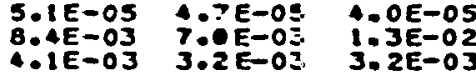

197

$1.0 \mathrm{~B}-0$.
$1.0 \mathrm{O}-\mathrm{O}$

$4.2 E-05$

$4.6 E-05$

$5.5 E-0.04$

$4: 0 E-05$

$1.4 E-02$

(

$2.96-03$

1: $A E-03$

$5.3 E-04$

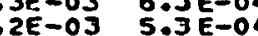

3:: $1 E-04$

B. BE 04

$2.4 E-03 \quad 5.2 E-0$

$\because: 1 E=0$

$3.9 E-04$
$1.7 E-04$

$2.2 E-04$ 1:JE-OA $3: B E-O 4$ 1:ZE-O

$7.9 E-04$

i:BE-04

$2.3 E-05$

$3.0 \mathrm{E}-04$

$2: 6 E-03$ I. $G E-03$

2.7E-0.

$2.0 E-04$

1.OE-OA A.Ge-O4

3.6E-O

$\begin{array}{lll}1: 6 E-04 & 6.7 E-05 \\ 2: 3 E-03 & 1.3 E-03\end{array}$

$1.2 E-028.1 E-04203$

$3.8 E-04$

$1.2 E-03$
$3.5 E-07$

2.7E-OT $1.9 E-07$

$6.1 E-07$
$2,1 E-0$,

S. $.0 E-0 E$

$2.8 E-07$
$1.5 E-0.05$

I.1E- OS

$\begin{array}{ll}8.8 E=06 & 2: 1 E E-0 S \\ 6.6 E-06 & 2.3 E-0 S\end{array}$

$\begin{array}{ll}6.6 E-06 & 2.3 E-0 . \\ 7.5 E-04 & 8.96-04\end{array}$

1.OE-O5 $2.9 E-05$

$7.1 E=04$

$2.0 E-03$

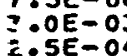

$1.2 E-05$
$5.5 E-06$

(1)

B. $5 E=0.96$

1.5e-O? 5.0E-OT

S.:DE-:

i. JE-OB

$3.0 E-06$
$6.6 E-07$

$5.3 E-O C$
$7: 2 E-0.02$

$1: 0 E=: 5$

6. 6E- 07

1.2E-05 $1.6 E-04$ a.6E-0

$1: 9 E-05$ 5. $2 E=06$

$\begin{array}{ll}1.7 E-05 & 1 \\ 2.4 E-05 \\ 2 E=04 & 2.4 E-04\end{array}$

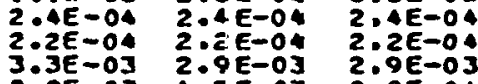

$202 E-04$

$2.2 E=04$

$3.3 E-03$

$3.3 E-04$
$8.25 E-05$

$1.0 E-03$
$1.0 E-04$

$1, \sum E-03$

. $2 E-04$

S.5E-OA

$2.4 E-04$

$2 \cdot 4 E-0$

$2.4 E-04$

$\begin{array}{lll}2.2 E-04 & 2.2 E-04 & 2.2 E-0 \\ 3: 0 E-03 & 3.1 E-03 & 2.8 E-03\end{array}$

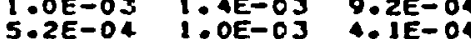

$8.0 E-05$

$\begin{array}{lll}1.2 E-04 & 1: 1 E-04 & 2.5 E-05 \\ 1.2 E-04 & 1: C E-O & 8.5 E-06\end{array}$

$\begin{array}{ll}6.0 E=02 & 6 . C E-O E Z \\ 1.9 E=02 & \text { i. } 9 E-02\end{array}$

6. IE-O2 5.9E-OS

B.IE-02 $3.6 E-05$

$1: 9 E-02$
$: 0 E-03$

.9E-02 $6.5 E-0 S$

$1.0 E-02$
$6.6 E-03$

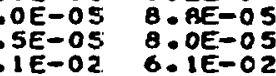

$1.9 E-0 z:$
$6.5 E-03$

$8.66=05$

$2.7 E-05$
$1: 1 E-O S$

$7: 5 E-03$

$1.0 E-02$
$6.0 E-03$

1E-03 $2.1 E-03 \quad 2.1 E-03$

. $5 E-03 \quad 3: 5 E-03$ 3:AE-0

0

$1.3 E-04 \quad 5.2 E-05 \quad 5.3 E-05$

8. BE-04 B.AE-04 B.2E-0.

$1.3 E-02 \quad 1.7 E-02$ 1.:3E-02

E.SE-04 5.:0E-04 5:1E=0.

$20 E-03 \quad 4.8 E-04 \quad 3.5 E-0$.

. $8 E-03$ I.5E-03 $1.6 E-03$

$.8 E-02$
$7.8 E-02$

$1.6 E-02$ 1.7E-02 $1.1 E-02$

T.A

$5.4 E-05 \quad 5.4 E-05 \quad 5.4 E-05$

5.5E-04 5.5E-04 S:SE-04

$2.5 E-042.6 E-04 \quad 3.5 E-04$

$6: 1 E-05$ 6:1E-05 $6: 1 E-05$

$\because A E-06$
$1: A E-05$

$1.4 E-05$ $4.9 E-06$ $1.98-06$

S.SE-04 5.5E-04 5.5E-D4

6.1E-03 $6.3 E-05$ : $: 5 E-1 E-03$

3.5E-03 $2.2 E-03 \quad 2.3 E-03$

$8.2 E=0$

$3.8 E-04$ 1:3E-OA $6: 2 E=04$

1.IE-04 $1.6 E-0$

$1.7 E-04$

1.7E-03

$6: 1 E-05$
$0: 0 E-04$

$1.1 E=04$

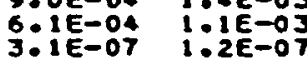

Q.OE-OT $2.7 E-O$

2.7E-0S $1.1 E-05$

$8.2 E-08$
S.

gE-04 $6.4 E=04 \quad 5.0 E-06$

6.6E-05 3:OE-04

1E-OS $1: 4 E-04$ 1.SEE-OS

.4E-04 6.2E-0J 2.4E-0.

.6E-03 $2.9 E-03 \quad 2.2 E=03$

:AE-03 $1.1 E-03 \quad 1: 3 E-03$

.3E-05 9.1E-05

$\begin{array}{lll}1: 0 E-04 & 4.9 E-05 & 7.3 E-05 \\ 0: 7 E-05 & 3.3 E-05 & 6.6 E-05\end{array}$

(1)

$6.6 E-05$
$6.1 E-0.02$

$0.5 E-0$
$1: 9 E-02$

$6: 9 E=-02$

$1: 9 E-02$
$6.3 E-03$ 
(REM,MICROCURIE)

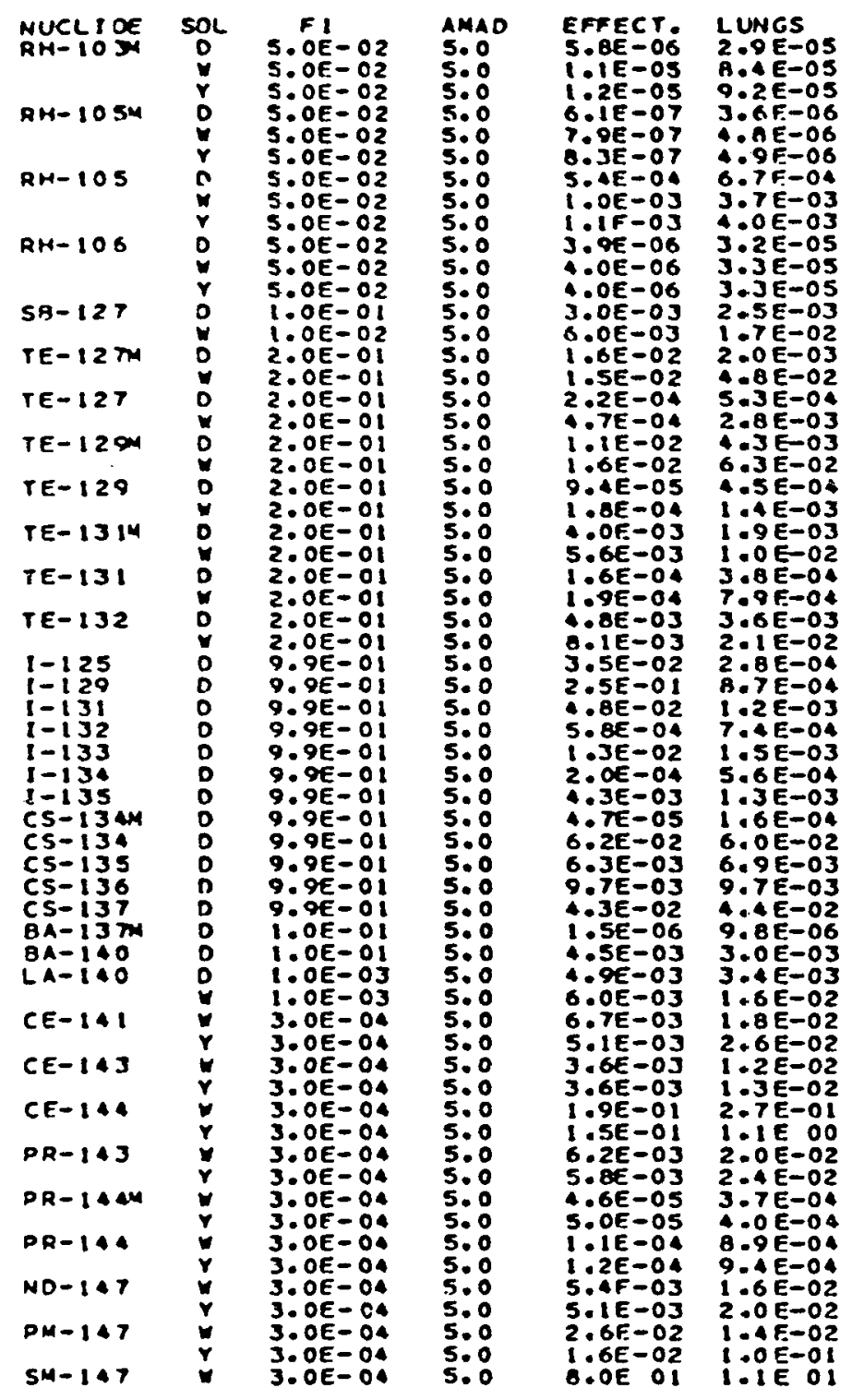

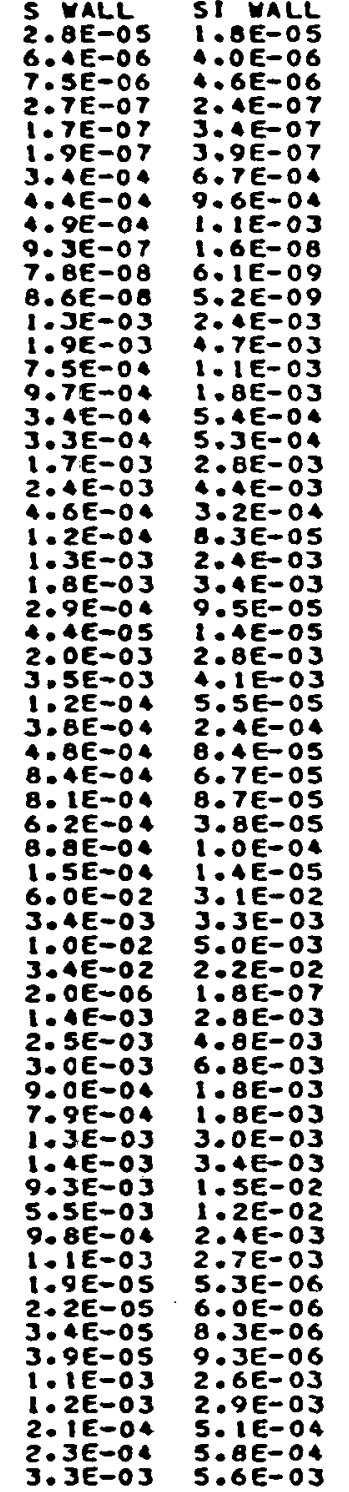

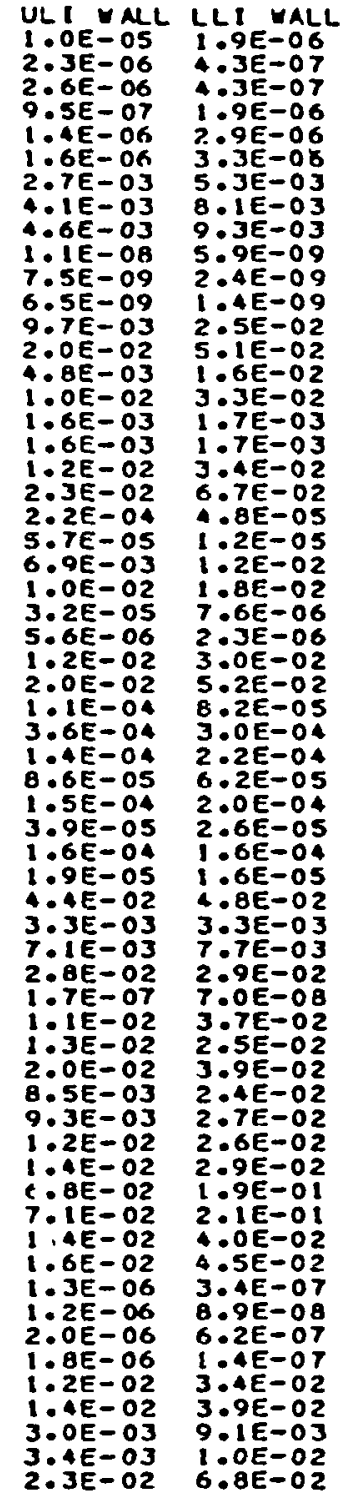

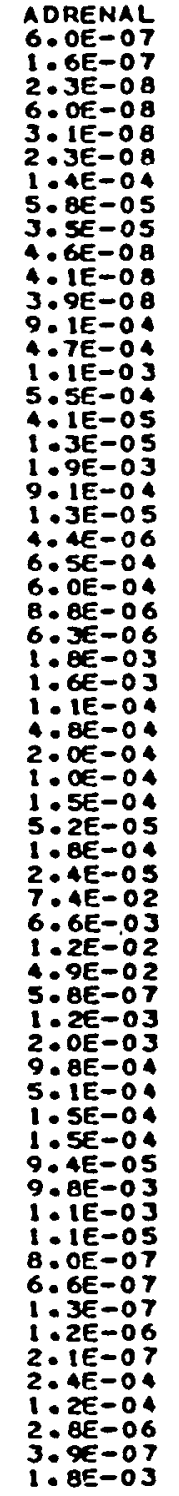

ic

1:SE=07

2. SE

$1: T E=08$

1.5e-04

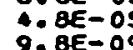

3. 4 E- -09

$9.5 E-0$

6. $1 E-04$

$5.2 E-0$.

I: $3 E-05$

$8: 5 E-04$

$1.2 E-05$
$3.2 E-06$

7. $8 E=04$

6. $5 E-04$

$2.3 E-06$
$2: 0 E-03$

$1: 9 E-03$

$1: 1 E=04$
$1: 9 E-04$

1.: $A E-04$

3. $3 E-04$

$3.3 E-05$
$1.0 .4 E-O S$

$2: A E-05$
$7: J E-02$

$6.6 E-03$

i. $9 E-02$

Y:IE-08

$1: 3 E=03$

$1 \cdot 1 E-03$

$1.2 E-04$

$1.6 E=04$

O:3E-03

$7.8 E-04$

:.1E-05

S. TEEO
$4.6 E-08$

:.1E-06

$8.6 E-08$
$1.6 E-0.8$

$1.6 E-04$
$1.5 E-04$

$2.5 E=06$
$1: 9 E=07$
$1.8 E-03$
KIONEYS LIVER
S.OE-OT $6.1 E-O T$

OSE-07 1.8E-07

$\begin{array}{ll}5.3 E-08 & 4.3 E-08 \\ .4 E-08 & 6.0 E-08\end{array}$

$\begin{array}{ll}3 E-08 & 3.3 E-08 \\ & \end{array}$

-AE-OA 1.AE-0A

$\begin{aligned} & 5.7 E-05 \\ & 3.5 E-05\end{aligned} 4.1 E-05$

. SE-OB $4.6 E-O B$

$17 E-08$ 3.BE-0B

$\begin{array}{ll}1 E-04 & 3.2 E-03 \\ : 6 E-04 & 1.0 E-03\end{array}$

$11 E-03 \quad 1.1 E-03$

:.2E-05 $4: A E-05$

.

. $6 E-04$ 8:8E-04

3.TE-00 $4: 5 E-00$

$5: 0 E-04 \quad 6.3 E-04$

8 . $2 E-06$ 6.5E-0

$\begin{array}{ll}9.9 E-06 & 6.2 E-06 \\ 0 E-03 & 1.6 E-03\end{array}$

$1.5 E-03 \quad 1.6 E-03$

. BE-0 4.6E-OA

$.6 E-04 \quad 1.8 E-04$
$0.9 E-05$

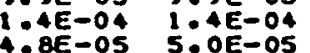

.7E-0. $1.7 E-0$

$\begin{array}{ll}2.4 E-05 & 2.3 E-05 \\ 7.4 E-02 & 6.7 E-02\end{array}$

:.6E-03 6.6E-03

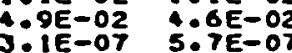

1 iE $1: 03 \quad 0 E-03$

$\begin{array}{ll}1: 9 E-03 & 1.6 E-02 \\ 1: 0 E-03 & 1.4 E-03\end{array}$

TSE 04

:OE-OA

$9.5 E=05$
$9.5 E=03$

$9.5 E-03$
$9.6 E-04$

5.8E-03

$3.3 E-04$
$3.6 E-07$

$6.5 E-08$
$5: B E-07$

$\because 1 E-07$
$: 9 \mathrm{E}-02$

9.7E 05

7 -06 $1: 1 E-0$

1.8E-03 $3.8 E$ OL
BREAST

6.2E-OT

$2.1 E-00$
$5.7 E-00$

TE-OA

$1.9 E-O A$
$5.6 E-05$

5.6E-0S

3. AE-OS

$2 . A E=08$

- उE-OA

-SE-OA

$5.5 E-04$

i. $3 E-05$

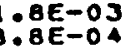

$1.3 E-05$

.6E-04

$4.0 E-06$

I:TE $\mathrm{Y}=03$

5.OE-OA

:IE-O 3

$9: 3 E-05$

$0 E-04$
$3 E-05$

$1 . B E-04$

$2.3 E-05$

$0.1 E-02$
$1.6 E-03$

:TE-02

$3.4 E-07$

$1: 1 E=03$
$1: 0 E=03$

$8.0 E-D A$

$2.9 E-O 4$
$1.1 E-O 4$

$1.3 E-04$

$9.5 E=05$
$8.6 E-03$

$8.6 E-03$

$9.5 E-04$
$1.1 E-05$

O.OE-07

i:2E-0?

i. IE-06

$1.8 E-07$

$1.5 E=04$
$1.1 E-04$

$2.6 E=06$
$3: 4 E=07$

$3: A E-0 J$
$1: 03$ 
INHALAT ION DOSES AT SO YEARS (REMPMICROCURIE)

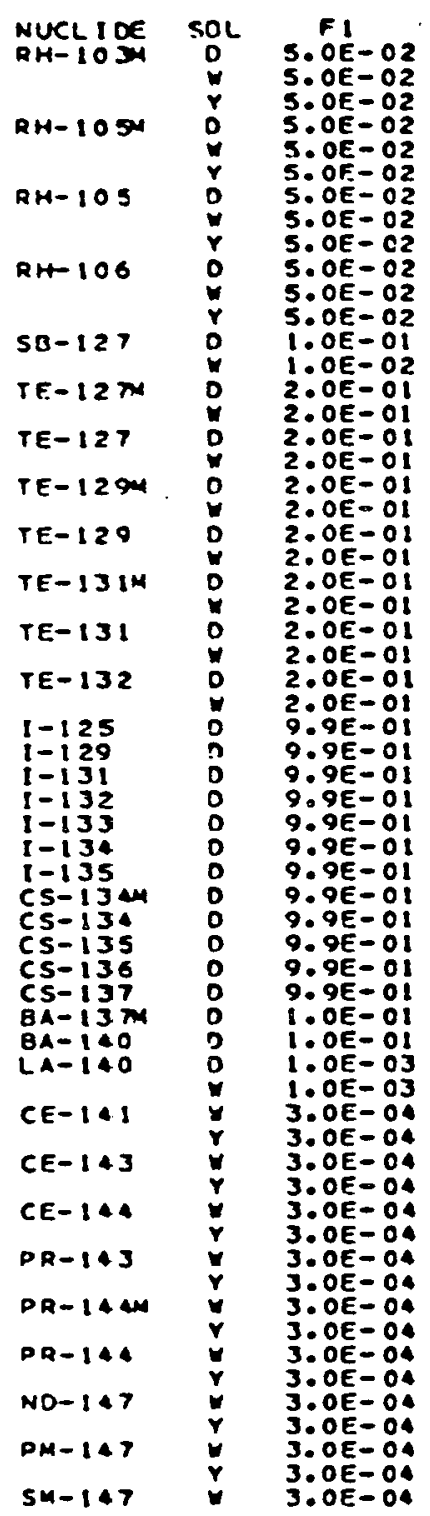

AM10
500
500
500
500
500
500
500
500
500
500
500
500
500
500
500
500
500
500
500
500
500
500
500
500
500
500
500
500
500
500
500
500
500
500
500
500
500
500
500
500
500
500
500
500
500
500
500
500
500
500
500
500
500
500
500
500
500
500
500
500
500

OVARIES PANCREAS BRAIN

$0.4 E-0$
$1.6 E-0$

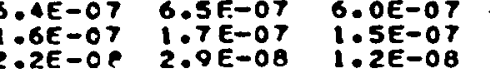

3.2E-0. $6.2 E-0 B$

$5.5 E-O B$ S.TE-OB

$1.5 E-04$

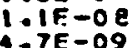

2. TE-O9

(.)

$2 E-03 \quad 1.1 E-03$

$.2 E-05$
$.4 E-05$

. $9 E-03$

$1.0 \varepsilon=03$

.

$8.9 E-04$
$1.4 E-05$

$\begin{array}{ll}1.7 E-03 & 7.3 E-04 \\ 7.7 E-06 & 1.3 E-05\end{array}$

$\begin{array}{ll}2.5 E-06 & 0.9 E-06 \\ 3.3 E-03 \quad 1.6 E-03\end{array}$

$4 . A E-03$

$6.9 E-05$

:.1E-0S

(1)

S. $7 E-02$

a.6E-OI

$4.2 E-02$

8.:BE-0日

$3.1 E-03$

A $A E-04$

$3.5 E-04$
$5.0 E-04$

$5.2 E-04$
O.SE -03

9.5E

$1: 1 E-05$

S. $B E-07$

S.2E $-0 E$
$1.1 E-06$

$1: 11 E-06$
$9: T E-08$

$1.6 E-04$

$\begin{array}{ll}3.1 E-07 & 3.9 E-07 \\ 1.8 E-03 & 1.9 E-03\end{array}$

$1: 1 E-09$
$1.3 E-04$

1. $2 E-08$

$3.6 E-09$

$1.8 E-0.04$
$1.2 E-03$

O.6E-04

3. $1 \mathrm{E}-06$

2. $6 E-0$

6. $8 E-06$

$1.6 E-03$

B. $1 E=04$

5. $1 E=04$

7:日E-05

1.TE-04

$1.7 E-04$

$6.7 E-02$

$6.6 E-03$
$1.0 E-02$

A. $6 E-02$

B. $5 E-083$

1.:DE-0.

i.ee-OA

T.TE-OS

$7.2 E-06$

$8: A E-03$
$T: A E-0.02$

I:IE-05

S.OE-0T

5. $0 E-08$

$9.6 E-08$

$8.9 E-06$

2:SE-06

$3.0 E-07$
$1.0 E-03$ a MAR ENDDST

6.2E-OT 6.OE-OT

$\begin{array}{ll}1.7 E-07 & 1.6 E-07 \\ 3.2 E-08 & 2.8 E-08 \\ 6.1 E-08 & 5.1 E-08\end{array}$

$\begin{array}{ll}6.1 E-08 & 5.1 E-0 B \\ 3.3 E-08 & 2.3 E-08\end{array}$

$\begin{array}{lll}1.3 E-08 & 3.3 E-08 & 2.3 E-08 \\ 1.1 E-09 & 2.5 E-08 & 1.6 E-08\end{array}$

1.IE-05 $4.8 E-05$

$2.8 E-08$

$2.0 E-0 B$

$2.3 E-03$
$6.7 E-04$

$5.6 E-042.8 \mathrm{E}-02$

$\begin{array}{ll}1.1 E-05 & 7.4 E-05 \\ 1.3 E-05 & 2.4 E-05\end{array}$

$1.9 E-03$ 3.BE-O

$2.6 E-05$

2. $A E-\cap B$

$1.6 E-O B$

$1.3 E-03$
$6 . B E-04$
$3.0 E-04$

I.IE-OI

$5.9 E-05$
$1.9 E-05$

:1E-05 $3.0 E-06$

1.2E-03

6.8E-06

$4.2 E-03$

$2.7 E-03$

$7.5 E=04$

$8.5 E-05$

$1.6 E-04$

$1.66-04$

$6.3 E-02$

$3.2 E-03$

3. $2 E-06$

$3.3 E=06$
$8.9 E-03$

$1 \mathrm{1E}-03$
$\mathrm{SE}-04$

$6.0 E=04$

6.3E-05

1.3E- O4

1. $0 E-O S$

$4.2 E-02$

$\begin{array}{ll}6.6 E-0 \\ 9.9 E-03 & 6.3 E-03\end{array}$

$\begin{array}{ll}4.2 E-02 & 3.0 E-02 \\ 3.1 E-07 & 2.3 E-07\end{array}$

$5.4 E-03 \quad 9.0 E-03$

$1.2 E-03 \quad 7.0 E-04$

$\begin{array}{ll}2.0 E-03 & 2.0 E-02 \\ 3.2 E-04 & 1.0 E-03\end{array}$

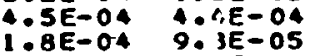

1. IE-01

2. $3 E=05$
$1.3 E-01$

SE-03

$1: E=03$

$5.5 E-07$
$1.2 E-07$

:3E-OY

$3.2 \mathrm{E}-07$
$3.2 \mathrm{E}-0 \mathrm{~B}$

$2.6 E-03$

S. IE-OO

B.

3.9E-0.3

$\because 22 E-01$
$\because \because 9 E-02$

i: $A E$ O 03
SKIN

6. OE -07
$1.5 E-07$

SPLEEN

1. $7 E-07$

6. IE-OT $1.5 E-07$
$1.2 E-08$
$1.1 E-08$ 5. IE- 08

5.0E-OB

6E-OB $8: 7 E-O 9$

$\begin{array}{ll}7 E-02 & 9200-02 \\ 300-02 & 020\end{array}$
$.3 E-05$
$1.9 E-05$

$0 E-O A$
$3 E-O B$

1
$: 1 E-08$

$2.8 E-04$

更

$4: 1 E-05$
$1.3 E-05$

$1.8 \mathrm{~B}-03$

R. $3 E=04$

3.AE-06

$3.6 E=04$

$7.1 E-06$

:5E-03

$1,1 E-03$
$2.7 E-04$

3.2E-OA I.BE-DA

B.2E-05

$3.6 E-05$

$2: 3 E-05$

$6.6 E-03$

$1,1 E=02$

$199 E-0$

$7: 1 E-04$

$2.1 E-04$

$4.5 E-05$

$3.5 E-05$

$8.4 E-03$
$8.2 E-04$

$1.1 E-05$

$8.00 E=07$
$6.0 E-07$

?:3E-08

1: 1 E-06

: $3 E-05$

2.5E-06

$3.0 E=07$
$1.0 E-03$

8 BE-O

$7: \begin{aligned} & \text { GE } 04 \\ & 1.0 E-05\end{aligned}$

(1)

$7: 0 E-02$

$1: 3 E=04$

$2.5 E-06$

$2.56-06$
$3.6 E-07$
$15-0 B$

$2 \cdot 3 E-O B$

$5: 6 E-08$
$7: 4 E-09$

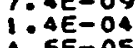

3. $Q E-05$ 2. 2 . $1 E-05$

C.AE-OB $8 . A E-09$

$\begin{array}{ll}3.7 E-08 & 2.6 E-09 \\ 0.0 E-10 & 08\end{array}$

1.2E-

$1.1 E-03 \quad 1.1 E-03$

$4.1 E-05$ S: $1 E-04$
. $1 E-05$

$\begin{array}{ll}A E-05 & 1.3 E-05 \\ 1.0 E-03 & 0.03\end{array}$

$5.2 E-04$

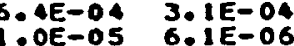

.

$\begin{array}{ll}1: 6 E-03 & 1: 5 E-03 \\ 1.6 E-03 & 1.0 E-03\end{array}$

$100 E-04$

$4.6 E-04$

$1.3 E-04604 E-05$

$1.2 E-04$

2.7E-05

$2.3 E-05 \quad 2: 1 E-05$

$6.6 E-03 \quad 6.6 E-03$

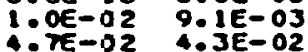

$6.2 E-07$ A.4E-0B

$1.0 E-03$

ZE-03 $3.9 E-04$

$\begin{array}{ll}1: 4 E-02 & 1: 9 E-04 \\ 1: 1 E-03 & 3.6 E-05\end{array}$

$2.4 E-03 \quad 7.4 E-05$

$\begin{array}{ll}2: B E-04 & 4 \cdot 1 E-05 \\ 9.1 E-01 & 0.3 E-03\end{array}$

7. $3 E-04$

$\begin{array}{ll}A . O E-077 & 8.0 E=07 \\ 6: B E-07 & 5.0 E=07\end{array}$

$1.6 E-07 \quad \because 3 E-08$

$1.2 E-06$
$2.5 E-07$

$4.2 E-05$
$4.4 E-05$

$2.5 E-06$

$2.9 E=07$
$1.8 E-03$

THYROID UTERUS

(

3.0E-OB $9: 2 E-09$ 2.4E-0B

5.8E-05 I.2E-05 8.3E-0S

$3.7 E-05 \quad 1.7 E-05$ 6.7E-0S

$6.0 E-08 \quad 2.0 E-08 \quad 1.0 E-08$

$6: 1 E-08 \quad 2.0 E-08$ 1:9E-09

$4.7 E-04$ 2.TE-D4 $7.7 E-0$

$1.1 E-03$ 1.IE-03 1.1E-03

$5.4 E-045.2 E-04$ 5.2E-0

4 .

1.3E-OS

OE-OA B.AE-OA

$1.3 E-05$
$5: 1 E-06$
$1.0 E-05$

$7: 1 E-04$
$1: 0 E-05$

$7.2 E-02$
$B: 1 E-02$

$3.1 E-03$

$14 E-03 \quad 3: 0 E-02$

$.6 E-03 \quad 6.8 E-03$

. $2 E-03$ 8. $3 E$ OD

$1.6 \mathrm{E} 00$

2:IE-04

5. SE-05

$2.1 E-05$
$5.9 E-02$

$4.2 E-01$

$2.1 E-03$
$1.3 E-01$

O.

$9.2 E-03$ 1:3E-02

(1)

B. $1 E=04$

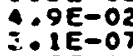

S.SE-04

$3.1 E-042.0 E-04$

$2.0 E-04$

$\because 9 E-05$
$6,7 E-O S$

2.9E-05

1: $1 E=03$

6. $0 E=07$

$2: A E-07$
$1: 3 E-06$
$3: B E$

$8.5 E-0$.

$8.0 E-07$

6.1E-07

$1.7 E=0.4$

1.1E=06

2.SE-06 2.5E-06 $4: 2 E-07$
$1.8 E-03$

I: $B E-03$

$1: 8 E-03$

(.)

(3E-06

$8.6 E-04$

$7: 4 E-06$

$2.0 E-03$

2. $0 E=03$

$\because 7 E-04$
i $3 E-04$

$3.0 E=05$

2.1E-0S 
(REM MLPCAOCOCRIE)

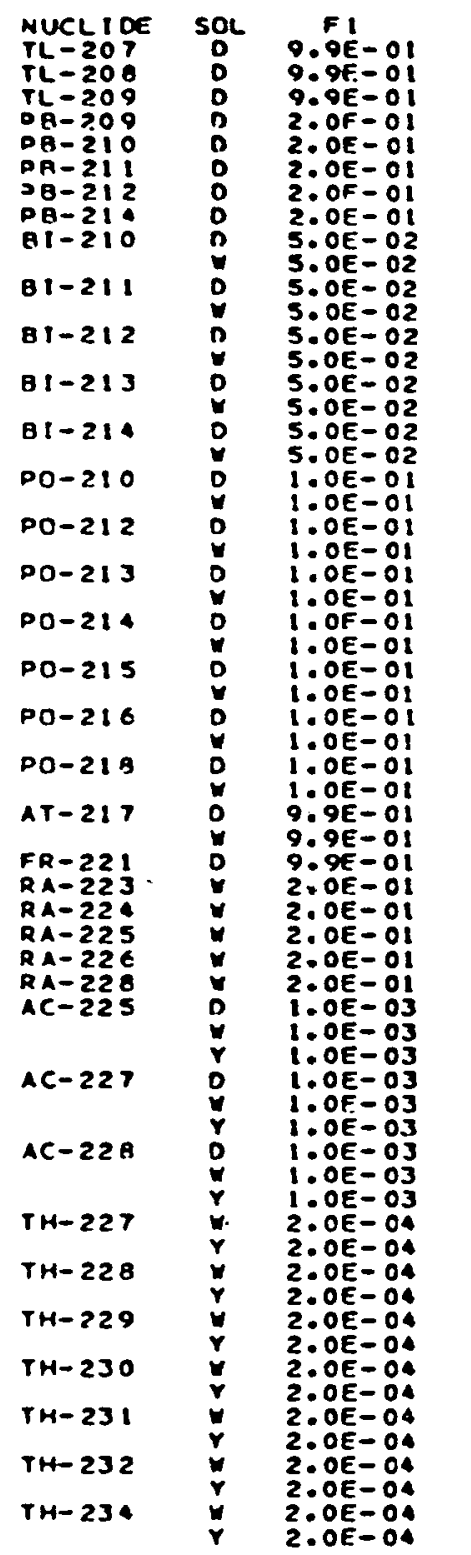

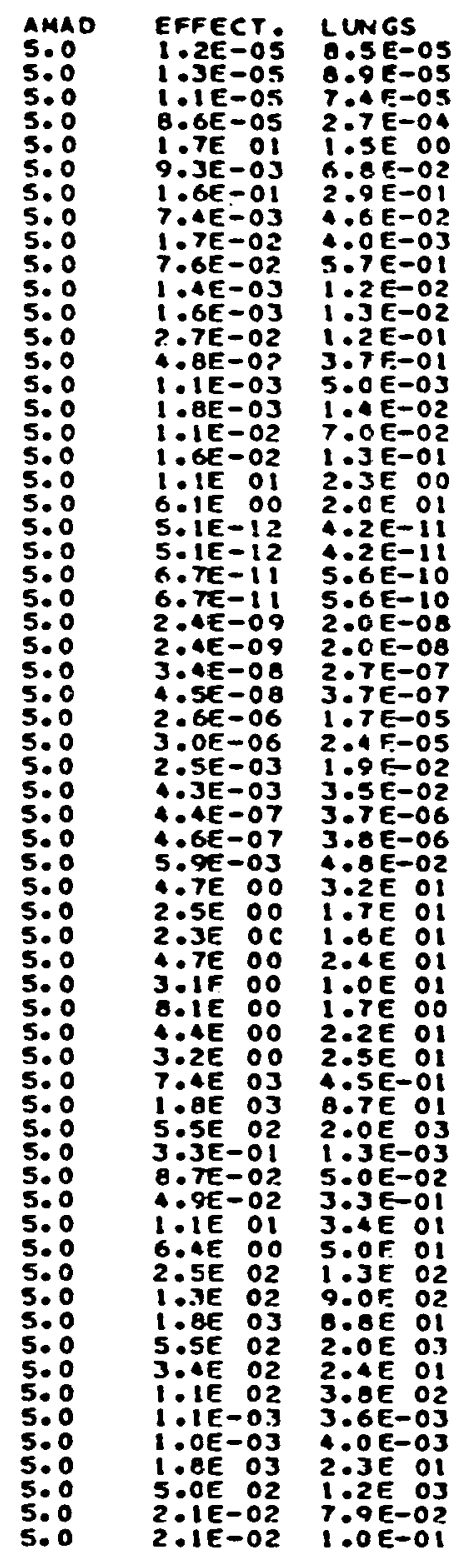

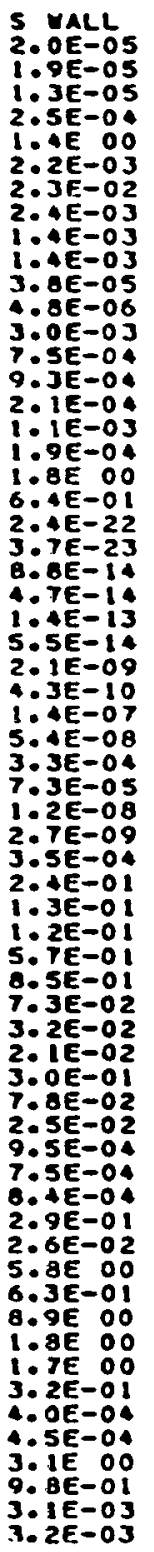

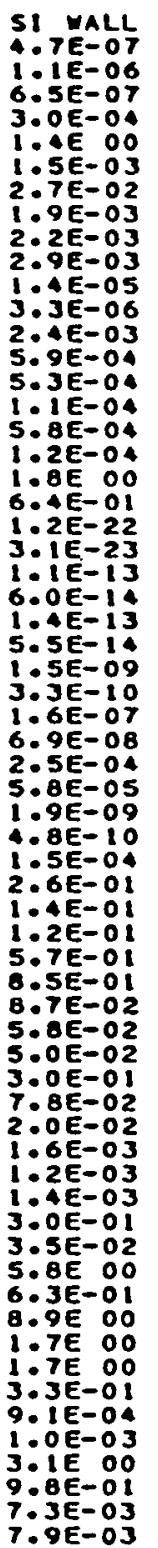

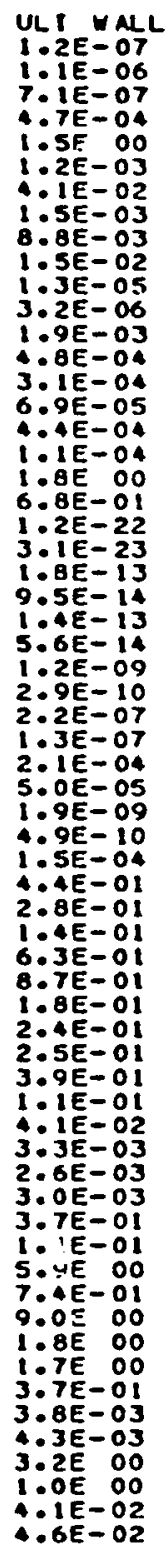

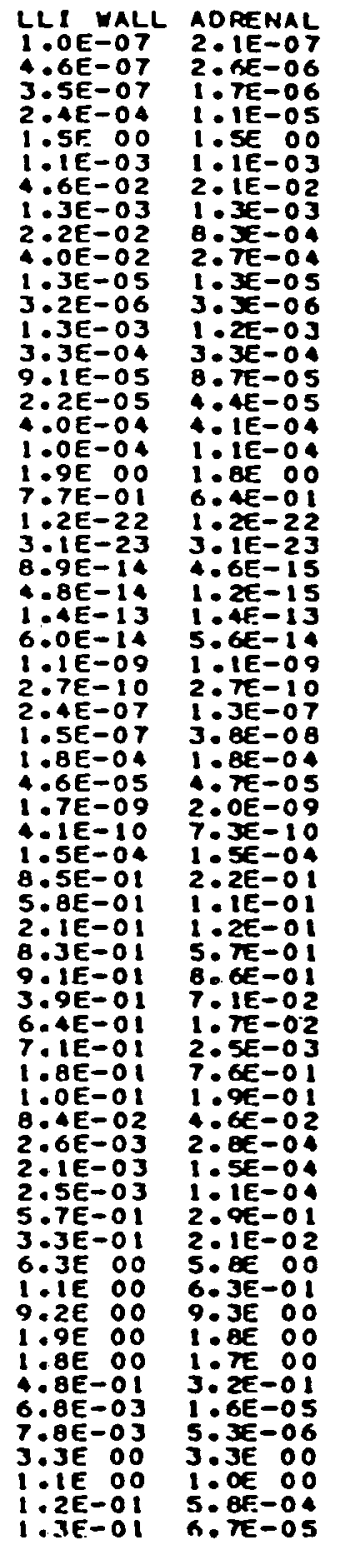

BL, 药LL

KroNers

LIVER

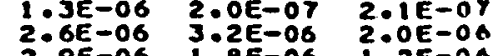
QREASY $4.5 E-07 \quad 2.9 E-06 \quad 1.8 E-06 \quad 1.2 E-000$ $1: 1 E-05$ 2.1E-05 $4.6 E-05$ 1:1E-0S $1.5 E 003.2 E 01$ 6.9E 01 1.SE 00

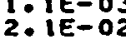
$3.7 E-04 \quad 7.9 E-04$ $1.2 E-012.6 E-01$ $1: 1 E-03$ 8 . $3 E-04$ $1.3 E-05$ 3. $2 E-06$ 1.2E=03 5. $A E-05$ $\because: O E-O A$ I:OE 04 $1.8 E-00$
$6.4 E-O 1$ $1.2 E-22$
$3.1 E-23$ $3.1 E-23$
$4.6 E-15$

$1: 2 E-15$

5. $6 E-14$

2. IE- 09

$1.3 E-07$
$3.8 E-08$

$4.6 E=05$

$1.8 E-09$
$\because .4 E-10$ $1.5 E-04$ $2.2 E-01$
$11 E=01$ 5. $7 E-01$ $6.5 E-02$ 1.6E 3.02 O. $Q E-02$ $3: 2 E-03$ 1.SE-04 $6.9 E-05$
$5.6 E-05$ $2.9 E-01$ S. $\theta E^{\circ}$. 6.2E- 01 9. OE $: 0$ 1.7E :0 $3.2 E-01$
$1.8 E-05$ $7.5 E-06$ 3.1E- 00 5. $6 E-04$
$6.4 E-05$ 
INHALATION DOSES AT 50 YEARS

(REMUACROCURIE)

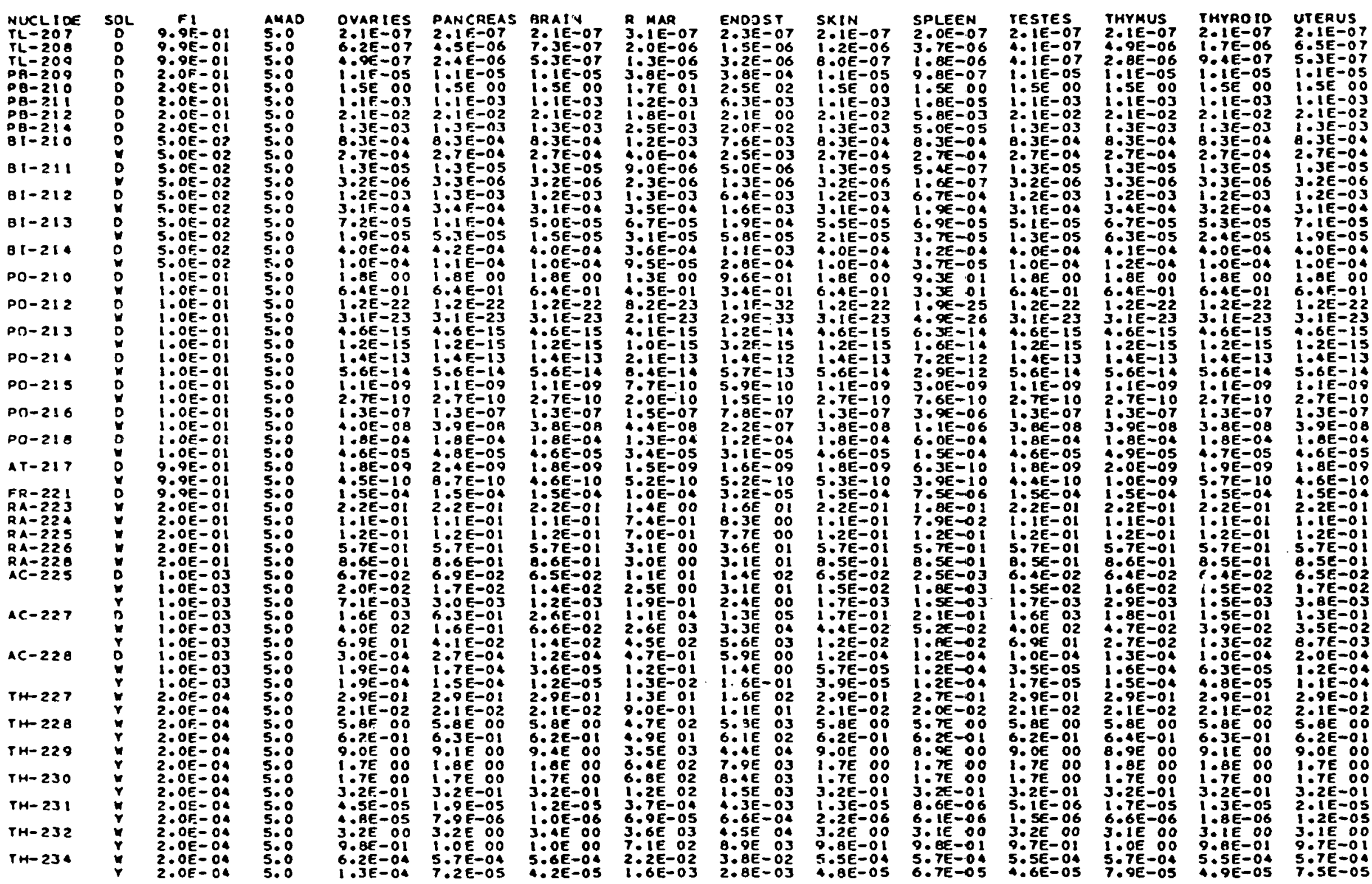




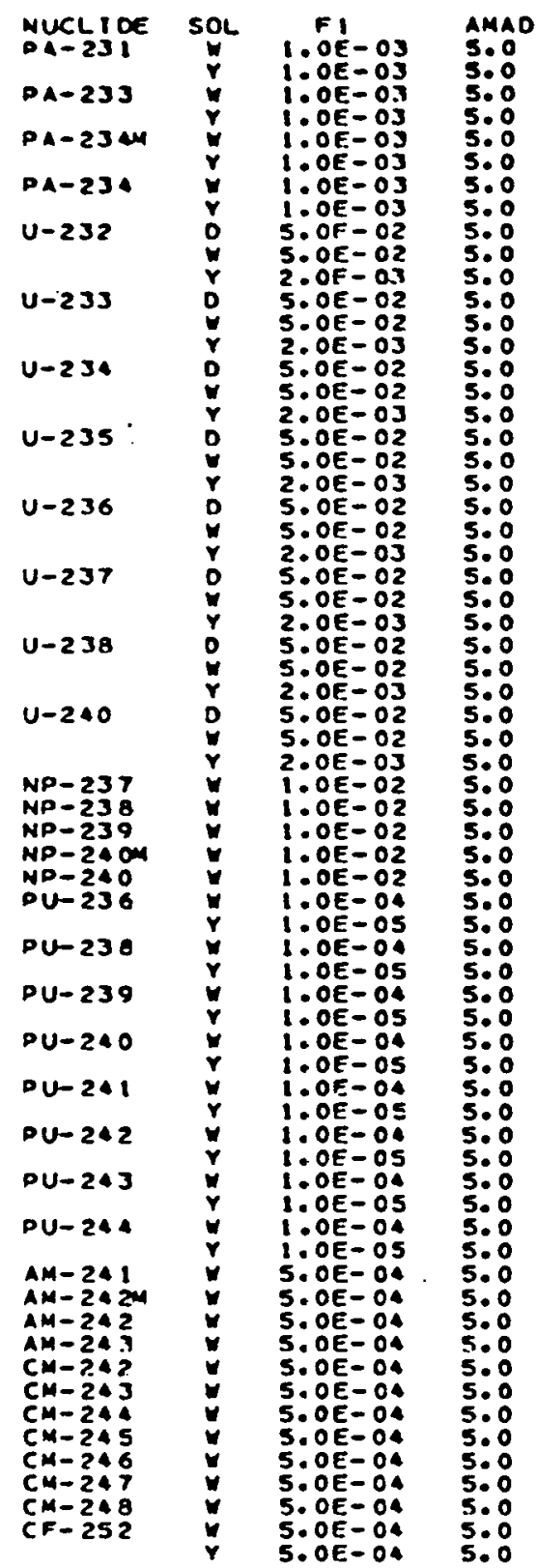

EFFECY: LUNGS 1.AE OJ $2.4 E$ OL

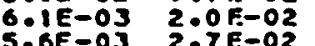

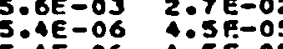
3.Ae-06

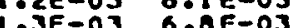
:AE OI B $8.4 E$ OO $3.5 F$ : 3. OE OO 5.5E-OI 3.7E : D 2:JE 01 3.96 OD $5.5 E-0$ $\because 7$ OI $3.9 E$ O2 $2.8 E: 50$ 5.1E-O .3E O1 $3.6 E$ O $3 . B E$ :O $5.2 E-01$ AE O1 3.TE O2 $3: 1 E-03 \quad 9: 6 E-03$ $3.1 E-03 \quad 1: 1 E-02$ $2.6 E$ O $\begin{array}{lll}1.2 E & 01 \\ 1.2 E & 03\end{array}$

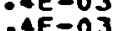
$2.66-03 \quad 1.3 \mathrm{E}-02$ S. $2.8 E-03 \quad 7.9 E-03$ $1: 8 E-04 \quad 1.2 E-03$ $1.8 E$ O2 $2.6 E$ OI

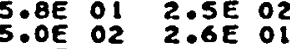
5.AE O2 A.2E O2

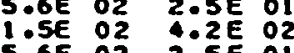
I.SE O2 A.2E O2 $1.1 E$ OI $1.0 E-02$

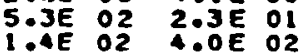
2:TE-O2 $1: 0 E-O 2$ $2.5 E-04 \quad 1.7 E-03$ 1.AE O2 $4.0 E$ OL S.6E O2 02 5.5E 00

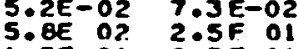
1.8E O2 2 2.5F $3.8 E$ O2 $2.8 E$ OI $3.0 E$ OL $2.8 F$ O

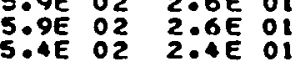
$2: 2 E$ O3 $1: 0 E$ O2 G.1E OI A.OE O2

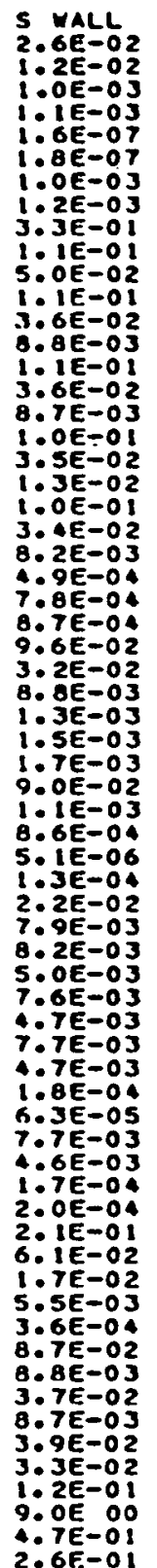

$$
\text { 更 }
$$

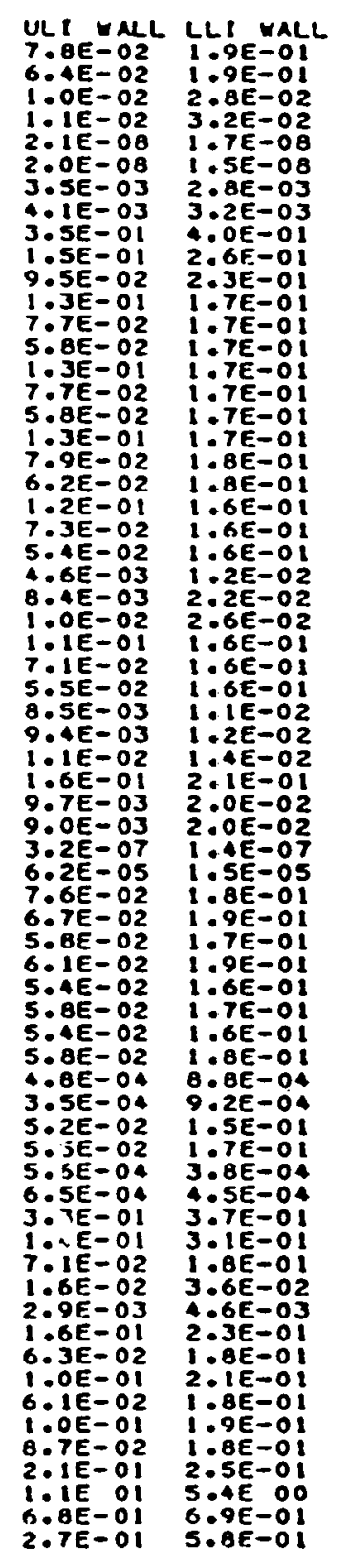

ADRENAL 1
$1: 0 E-02$
3 3. $A E=04$ $1.3 E-0 B$ $6.3 E-09$ $2 \cdot 3 E-04$ 3.4E-O 1:1E-01 1. IE-OI 5 . $1 E-03$ 1. $1 E-01$ 5. $1 E-03$ $1: 1 E=01$ $1: \begin{array}{ll}1 E-02 \\ 1: 0 E-01\end{array}$ $1.0 E-01$
$3.1 E-02$
$3.1 E-02$

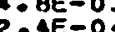
$1 . A E=04$ $9: 3 E-05$ 3. $O E-02$ $1: 6 E-04$ $6.1 E-05$ 2. IEE-DI $2: B E=04$ $1.1 E-06$ 3. $9 e-02$ 7: $60-03$ 5.:0E-03 A. $35=03$

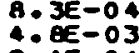
$9: 1 E-0$ $\triangle B E E=04$
$1.0 E-0$ 5. $6 E-03$ 5: $1 E=03$ 3. $0 E-06$ $1: 2 E-01$ 1. $5 E-02$ $2: 9 E-02$
$1: 5 E-02$ i. $4 E-05$ 1:AE-OS \begin{tabular}{ll}
$1.9 E-01$ & $2.9 E-02$ \\
\hdashline$B E-03$ & $4: 7 E-03$
\end{tabular} $7.2 E-02$ 5. $2 E-03$ 7. TE-OL $3 E-02$ .OE-O $1.3 E-02$ $2.4 E$ OI $3.0 E-00$ 3. $2 F-01$ 3. $4 E-02$

2. $11 E-02$ $2.6 E-: 0$

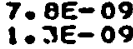
1. 3E- 04 1:Oe ol 1 . IE- 01 S. $1 E-03$ 1. $1 E-01$ 1. OE 01 5. $2 E-03$ $1.0 E-01$
$3.1 E-02$ 4 OE 03 $1.6 E=04$ $2.9 \varepsilon-02$ I. $6 E-0$. 5. $2 E-05$ 2. $7 E=04$ $6: 9 E-06$ $1: 0 \mathrm{E}-03$ A. $A E-0.4$ B. $2 E=03$ i. $2 E=03$ 7: $7 E=04$ 1. $0 E-05$ IE-03
KIONEYS LIVER

$3.3 E 00$ 2.1E 00

GREAST $2 \cdot 2 E-03$ $3.2 E-09$ $2.9 E-09$ 1 . BE-OA i:ge ol 1.96 OI S.TE - OO 1 . BE Q.TE-OI

I. TE OI

B.ie-ol $1: T E$ : 8:2E-O1 $2: P E=03$ 1.6E :I 5. $\mathrm{OEE}-01$ 3

1. $2 E-04$ $2.7 E-04$ 5.9E-05 $1: 0 E-05$ $2.3 E-02$

\begin{tabular}{l}
$4.6 E-03$ \\
\hdashline $.6 E-03$
\end{tabular}

$8.4 E-04$
$\therefore .3 E-03$ $8.0 E-04$ $4.4 E-03$
$8.2 E-04$
3 $3.5 E-04$ 7.3E-0S $5.0 E-03$
$9.4 E-0.06$ $4: 5 E-06$

$41 E-01$

Q. $2 E-02$

$1: 0 E-02$

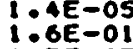
$4.7 E-03$ 5.0E -03 $6.7 E-02$
$6.0 E-02$ $2 \cdot 2 E-0$ 2.0E-D1 I.BE-OI
$1.0 E-0$

C.3E-O 2 CE-O

6.4E-09 $1.0 E-08$ 3.IE-OA i:TE-OA 3.5E-04 $1.6 E-0$. $3.16-01 \quad 3.4 E-01$ $4.8 E-02$ a.0E-02 101 5.1E-03 5.2E-0 $1.1 E-01$ I.IE-OL $5.1 E-03$ S.1E-0 $\begin{array}{ll}8.6 E-02 & 1.0 E-01\end{array}$ $1.0 E-02$ B.TE-03 3.1E-02 3.1E-02

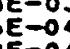
$1.3 E-04$ 1:2E-04 1. $2.5 E-02 \quad 3.0 E-02$ 6.3E-05 $1 . A E=0$. T.0E-05 5.3E-05 $2.2 E-0$ $2.1 E-03$ B.6E-0S $\begin{aligned} & 3.8 E-06 \\ & 7.9 E-05\end{aligned} \quad 2: 0 E-05$ T.OE 02 1.TE-O2 $1 \cdot 1 E \quad 02 \quad 3.3 E=03$ $3.4 E$ O2 $9.0 E-04$ $38 E$ O2 B.2E-OA 2.1E O3 A.AE -03 $3.8 E$ O2 $0.7 E-04$ 7 . $2.0 E$ O3 $A .5 E-03$ 2.0E-04 $4.0 E=06$ 2.2E-06 $2.0 E$ O3 $2.2 E-01$ $2.2 E$ O3 $1: 3 E=02$ 1.9E-OL $1.4 E-O 5$ 2.26 OJ $7.7 E-02$ $1.5 E$ O3 $3.0 E-02$ 2.JE 03 3.AE-O2 2.1E O3 $1:$ IE-0 $\begin{array}{lll}8.3 E & 03 & 1.0 E-D \\ 5.3 E & 02 & 1.0 E-0\end{array}$ G.2E OI $1.0 E-D$ 
INHALAT ION DOSES AT 50 YEARS

(REMIMICROCURIE)

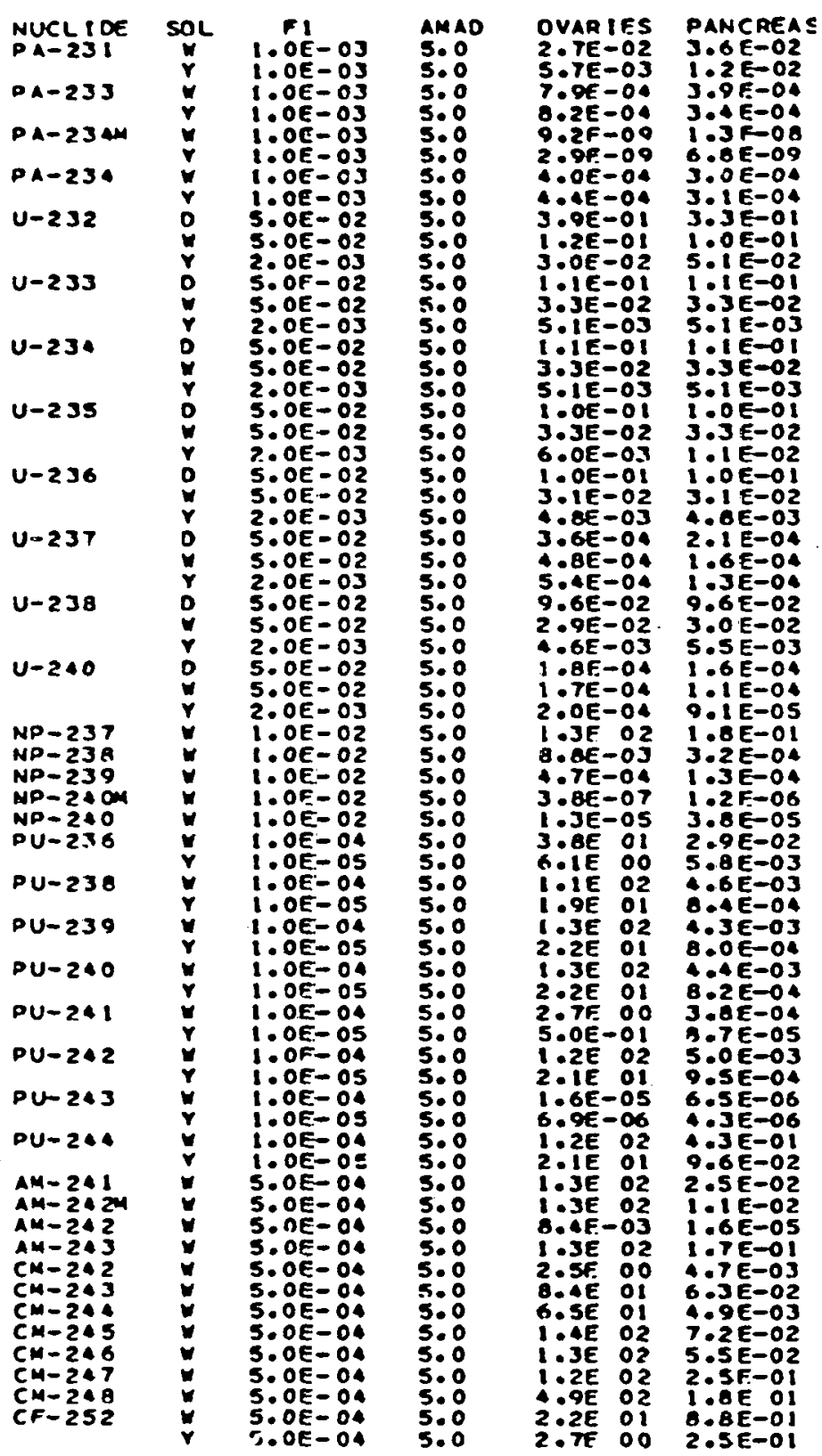

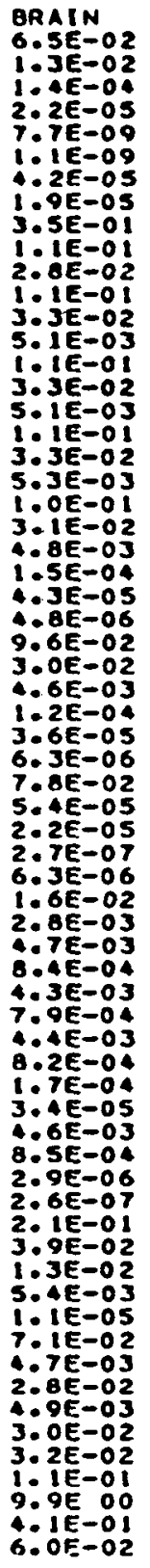

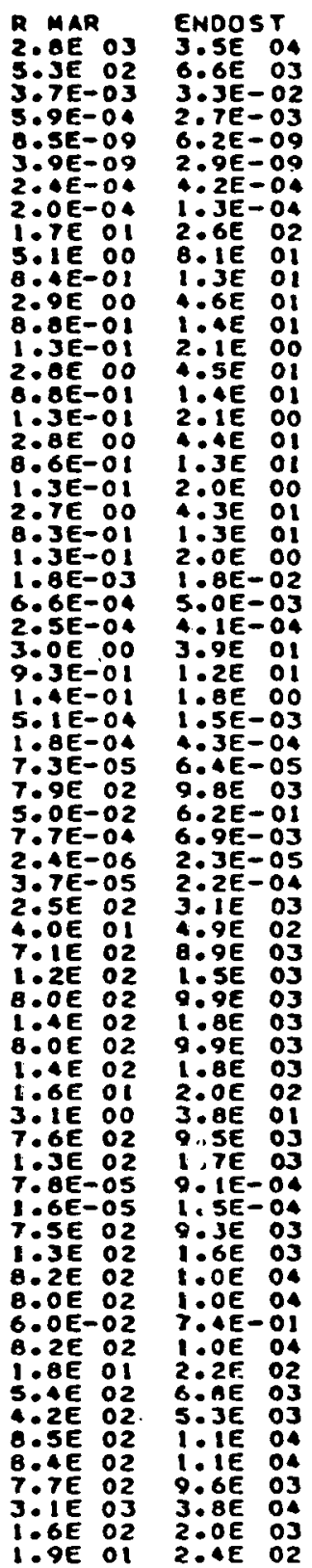

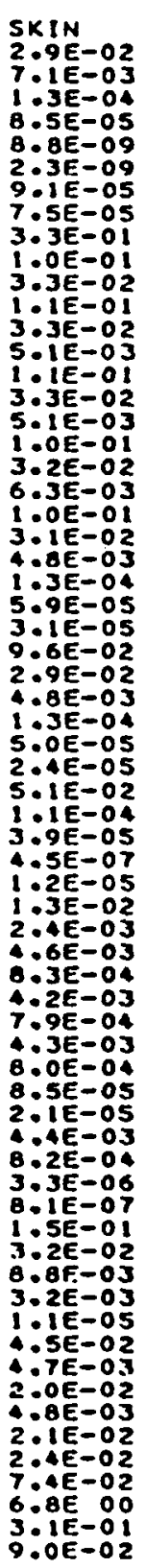
$8.5 E-03$ $2: A E-O 4$ $1: 3 E=0 B$ 6. $1 E=09$ $2.5 E-04$ $3, T E=02$
$9,7 E=02$ $\therefore \in E=02$ 1. $\begin{array}{ll}1 F=01 \\ 3.0-02\end{array}$ S: $: E=03$ 3: $3 E-02$ 5.1E-03 $3 . x \leq-02$ $\begin{cases}1 E-02 \\ : 0 E-01 \\ 0\end{cases}$ $1: 0 E-01$
$3: 1 E-02$ $\triangle B E=0 J$
$2.0 E=0$ I. $1 E=04$ $1.1 E-04$
$9.6 E=02$ $2, S E=02$ $1: 60=04$ $9.32-05$

6: $1 E-02$

$2.6 E-04$

3. $2 E-05$

$2.3 E=03$

$\therefore: \because 5 E-03$

$8.4 E-04$
$4.2 E-03$

$A 3 E=03$

1. $0 E=04$

$3 . \frac{2 E-05}{3 E-03}$

5.:

3: उE-06 2:OE-06

Q.:SE $=02$

9.6E-OJ

$1: 4 E-05$

S.SE-02 1.JE D2

$2: 3 E-02$
$A, B E-03$

$2 \cdot 5 E-02$

$8.36-02$

$3.5 E-0.0$
$3.5 E-01$
$1.8 E-O 1$

6.4E Ol

$1: 4 E$ O2

4 .9E 02 2. JE OI
THYMUS

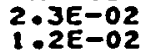
$3.3 E-04$ J.TE-OA $1,7 E-08$ $2: 0 E-08$ $300 E-04$

$1.0 E-01$

6. $B E-02$

$1: 1 E-01$
$3.3 E-02$
$101 E-03$

$5 \cdot 2 E-03$

$3: 3 E-02$

$5.1 E-03$
$1.0 E-01$

J. $2 E-02$

1.:5E-02

$3: 1 E-02$

$1,2 E-04$

$1.2 E=04$

9. $6 E=02$

$6.3 E-03$
$1.3 E-04$

$9: 5 E=05$

$7.3 E-05$
$5.3 E-02$

$2.5 E-04$

$1.6 E-06$

$4: 7 E=05$

$3.5 E-03$
$4.5 E-03$

$4.5 E=03$
$8.2 E=04$

$4: 2 E-03$
$7: 9 E-04$

$4.2 E=03$

B. OE-OA

$3: 3 E-05$

$8.6 E-04$

$1.3 E=06$
$1.2 E-0.1$

S.AE-OI

$8.0 \varepsilon-03$

$1: 5 E-05$

$: 5 E=02$
$4 . T E=03$

2.:OE 02

$2.1 E=02$

$1: 9 E-02$
$8.7 E-02$

5.3E OO

I.TE
$2.9 E-01$
THYROIO UTERUS.

THE-OZ 2.AE-0

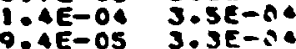

1.OE-OB B.AE-SO

1.6E-09 $1: 9 E-B$

9.5E-05 2.SE-E

$1.0 E-01$ l.oe-O

MYE-O2 2:YE-0:

$5.3 E-02$ J.JE 02

1. IE-01 $1 \cdot 1 E-01$

S.1E-03 5.1E-03

3.:2E-02 3.:2E=1

. OE-03 5.AE-03

$3.1 E-02 \quad 3: 1 E-02$

$\begin{array}{ll}1: 3 E-03 & 1.8 E=03\end{array}$

S. SE-OS 2.2E-OA

$9.6 E-02$ 9:5E-02

5. $0 E-03 \quad 2.9 E-02$

$1.2 E-04$ 1.8E-0.4

S.2E-0S $1.1 E-04$

.6E-02 4.0E-02

1:0E-04 $1: 1 E-0$.

6.3E-07 2.4E-O?

$1 . B E-05 \quad 1: 0 E-05$

$1,7 E-03.1: B E-03$

M.

$4.2 E-03 \quad 4.2 E-03$

$2.2 E-03 \quad 4.2 E-03$
$3.0 E-04$

$6.0 E-05$ 5.0E-OS

$1.3 E=5$

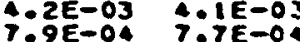

$3.5 E-06$ 5.2E-OS

i:AE-07 $3: 1 E=06$

$2.8 E-02 \quad 1.8 E-02$

$2.1 E-03 \quad 7.0 E-0 J$

-1E-0S $1: 7 E-05$

3. $9 E-02 \quad 3.7 E-02$

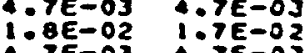

$\because .7 E-03 \quad A .7 E-03$

$\begin{array}{ll}1: T E-02 & 1.8 E-02 \\ 1.6 E-02 & 1: A E-02\end{array}$

A.JE OO J AE OO

$1: 9 E-O 1$
$1: B E-02$ I:BE-OI 
INCESTION OOSES AT 50 VEARS

(AEMPMICROCURIE)

\begin{tabular}{|c|c|c|}
\hline $\begin{array}{l}N U C L 1 O E \\
N=3 \\
C-14 \\
N A-22 \\
D=32 \\
X-40 \\
Y N-54 \\
M N-56 \\
F E-55 \\
F E=59 \\
C O-5 Y\end{array}$ & 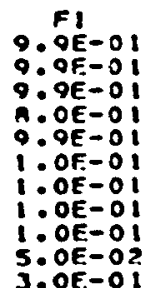 & $\begin{array}{l}0 . \\
1: \\
1: \\
1: \\
20 \\
\text { s. } \\
5 .\end{array}$ \\
\hline $\mathrm{CO}-5 \mathrm{~B}$ & $E-02$ & \\
\hline CO-60 & $O E-02$ & \\
\hline $\begin{array}{l}2 N-65 \\
R A-86 \\
S R-B Q\end{array}$ & $\begin{array}{l}0 E-0 \mid \\
0 E-0 \mid \\
0 E-0 \mid \\
0 E-0 \mid\end{array}$ & \\
\hline SR- 90 & $O E-O I$ & \\
\hline SR-QI & -0 & \\
\hline $\begin{array}{l}Y-90 \\
Y-91 M \\
Y-91 \\
2 R=95 \\
2 R=97 \\
N B-95 M \\
N A-95 \\
N R=97 M \\
N B-97 \\
M O-99\end{array}$ & 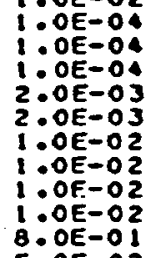 & \\
\hline $\begin{array}{l}\text { TC-99M: } \\
\text { TC-99 } \\
R U-103 \\
R U-105 \\
R U-106 \\
R H-1034 \\
R H-1054 \\
R H-105 \\
R H-106 \\
S A-127\end{array}$ & 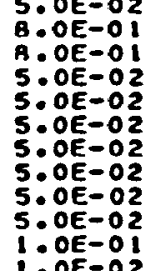 & $\begin{array}{l}1 \\
2 \\
5 \\
6 \\
6 \\
9 \\
9\end{array}$ \\
\hline 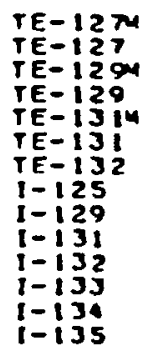 & 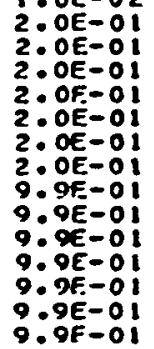 & 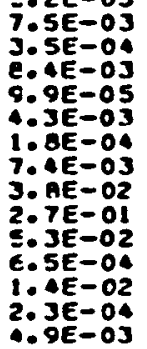 \\
\hline
\end{tabular}

LUNGS

B. AE-OS

YALL

1. IETOOA

$1.0 E-0.2$

$1.2 E-03$
$1.2 E-02$

$3.5 E=03$

-. 2E-04

-

2. 5E-0J

6. $9 E-O A$

3. 0 E- 04

1. AE-03

$2.1 E-02$

2E-0

9. $\triangle E-04$

3. 1 E- 05

$1: 7 \in-04$

1.2E-:

S. $1 E-0 B$

A. $7 E=06$

B. 7 E-OS

O.

证

3.2E-07

7. $3 E-06$

5. $8 \varepsilon-05$

1.

$2.0 E-04$

2. $3 E-05$

1.9E-08

1. $3 E-08$

$3.2 E-08$

5.6E-04

3. TE-OA

1. $2 E-0 S$

$1.9 E-06$

. AE-06

$6.5 E-04$

$1.5 E-04$

$6.1 E-04$

9. $8 E-05$

$\because .6 E-05$
$: 0 E-04$
SI YALL ULI WALL LLI WALL

9.0E-OS I.IE-OA 1.AE-OA

S. TE-03

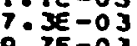

$8.6 E-03$

6. 9 E- 05

BL YALL

KIONEYS

8.6E-05
$1.1 E-03$

LI VER

2.7E-03 $9.7 E=03$

उ.AE-03

S. $1 E-0 j$

$1.9 E-02$
$1.4 E-03$

$1.1 E=02$
$2.4 E=03$

$1.9 E-02$

$1.2 E-0$.

$4.0 E-04$
$3.6 E-03$

1: $9 E-04$

$7.7 E-04$
$B . O E-0.4$

$2.3 E-03$
$5.4 E-03$

$1.6 E=02$
$0.2 E=03$

9. AE $1 E A$

5. OE-03

1. $T E-0$.

2. $0 E=04$

$5.1 E-08$
$1.4 E=05$

2. $3 E=06$

$\because: 1 E=04$

7. $6 E-07$

2. $5 E-05$

6. $5 E-04$

2. $0 E-05$

$5.1 E-04$

5. $1.0 E-03$

$1 . B E-08$

$2.0 E=08$
$4.0 E-05$

5. $\theta E-0 B$

3. $2 E-04$

3. $7 E-04$

1.:2E-DS

3. $4 E-06$

i.1E

i: $3 E=03$

5. $2 E-04$

$1.7 E=04$

i. $6 \mathrm{E}-04$

$6.8 E=05$
$2.0 E-04$
8. 3E-OS $9.9 E-03$

1: $9 E=02$

$9.4 E-05$

5. TE 03

3: $2 E=04$

9.0E-OA

$3.7 E-03$
$B .5 E=03$

$1.3 E=02$
$8.2 E-03$

9.4E-OA

5:OE-OJ

I:TE $=04$

$9.1 E-06$

$2 \cdot 3 E-05$

2.9E

2. $9 E-05$

3. IE

:. $6 E=05$

$9: B E-03$

6. $6 E-04$

3. OE-OA

7. $22 E-05$

$1.9 r-08$

$1.9 E-08$

3. $6 E-0 B$

2.TE-O4

$3.6 E-04$

l.2E-0S

$2.6 E=06$

i.OE-OS

2. OE -04

S:OE $9=04$

$1.0 E-04$

S. $2 E=05$ onés.

$1: 0 E-03$

2:AE-03

$1.0 E-02$

$1: 16 E=05$

(3)

i: $P E-O 4$

6.AE-OA

:.9E-03

2.66 $2 \mathrm{E}=03$

$1.4 E=02$

$9.4 E-04$

S. $1 E-05$

S.OE

2:0E 1.04

5.1E-0.8

$6.8 E-0.6$

$3.1 E-08$
$3.9 E-04$

$3.0 E-04$

3. OE-OA

3. SE-O?

$1.2 E-05$

$7.3 E-04$
$1.3 E-O 4$

1:5E-05

:BE-04

$6.0 E-05$
$5.6 E-03$

$3.2 E-08$

3. $9 E-08$

2. $5 E-0 B$

$2.8 E-04$

3: $8 E-04$

$1.2 E-05$

$2.4 E-06$

S: IE-DA

$1.2 E-03$
$5: 5 E-04$
$0.2 E$

1. $2 E-0 J$

\begin{tabular}{ll}
$66-04$ \\
\hdashline $0 E=04$
\end{tabular}

i:ge-04

$\because \because 7 E-05$
$i .9 E-0.04$ 
INGESTICN DOSES AT 50 VEARS

(REMIMICROCURIE)

\begin{tabular}{|c|c|c|c|c|}
\hline $\begin{array}{l}N U C L I O E \\
H-3 \\
C-14 \\
N A-32 \\
D-32 \\
X-40 \\
Y N-54 \\
Y N-56 \\
F E-5 S \\
F E-S O \\
C O=57\end{array}$ & 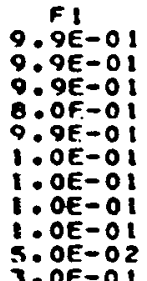 & $\begin{array}{l}\text { OVARIES } \\
8.3 E-05 \\
7.4 E-04 \\
9.0 E-03 \\
2.0 E-03 \\
1 . A E-02 \\
3.5 E-03 \\
3.1 E-04 \\
3.9 E-04 \\
5.8 E-03 \\
6.5 E-O 4\end{array}$ & $\begin{array}{l}\text { PANCRE AS } \\
8.1 E-05 \\
1.1 E-03 \\
1.0 E-02 \\
2.0 E-03 \\
1.9 E-02 \\
1.3 E-03 \\
2.1 E-04 \\
4.1 E-04 \\
3.3 E-03 \\
1.9 E-O 4\end{array}$ & 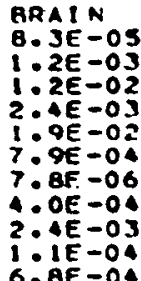 \\
\hline$C O-5 A$ & & - & & \\
\hline $10-60$ & & $E-1$ & & \\
\hline $\begin{array}{l}2 N-65 \\
R R-86 \\
5 Q-B 9\end{array}$ & $\begin{array}{l}5.0 E- \\
9: 9 E \\
3 . O E-\end{array}$ & $\begin{array}{l}1.2 E-02 \\
e .1 E=03 \\
9.4 E-04\end{array}$ & $\begin{array}{l}1.2 E-02 \\
8.1 E-03 \\
9.4 E-04\end{array}$ & $\begin{array}{l}5 E-02 \\
2 E-03 \\
E-04 \\
E E-0 .\end{array}$ \\
\hline SR-90 & $E-0$ & $\begin{array}{l}\text { S. } 0 E-03 \\
1 . T E-04\end{array}$ & $\begin{array}{l}0 E-03 \\
7 E-04\end{array}$ & \\
\hline $52-91$ & $O E-02$ & $\begin{array}{l}3 E-04 \\
E=-04\end{array}$ & $\begin{array}{l}E-O 4 \\
E-O 4\end{array}$ & \\
\hline $\begin{array}{l}Y=90 \\
Y=91 M \\
Y-91 \\
Z R-95 \\
Z R-97 \\
N B-95 M \\
N B-95 \\
N B=97 M \\
N B=97 \\
M O-99\end{array}$ & $\begin{array}{l}1.0 E-04 \\
1.0 E-04 \\
1.0 E-04 \\
2.0 E-03 \\
2.0 E-03 \\
1.0 E-02 \\
1.0 E-02 \\
1.0 E-02 \\
1.0 E-02 \\
8.0 E-01\end{array}$ & $\begin{array}{l}5.7 E-08 \\
2.6 E-05 \\
1.3 E-05 \\
3.0 E-03 \\
2.3 E=03 \\
3.4 E-04 \\
3.0 E-03 \\
9.0 E-07 \\
5.4 E-05 \\
8.2 E-04\end{array}$ & $\begin{array}{l}\text { S. IE-08 } \\
4.1 E-05 \\
2.1 E=06 \\
3.9 E-04 \\
4.0 E-04 \\
4.1 E-05 \\
4.1 E-04 \\
2.9 E-06 \\
6.1 E-05 \\
8.8 E-O 4\end{array}$ & $\begin{array}{l}-08 \\
-08 \\
-07 \\
-05 \\
-06 \\
-06 \\
-05 \\
-09 \\
-07 \\
-04\end{array}$ \\
\hline 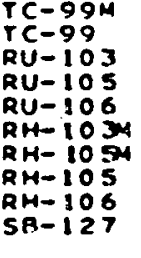 & $\begin{array}{l}-01 \\
001 \\
0-02 \\
0-02 \\
-02 \\
-02 \\
-02 \\
02 \\
02 \\
0.02 \\
-01\end{array}$ & $\begin{array}{l}3.4 E=05 \\
2.3 E-04 \\
2.1 E-03 \\
3.6 E-04 \\
E .2 E=03 \\
1.5 E-07 \\
7.9 E=08 \\
2.1 E-04 \\
1.4 E-08 \\
2.2 E-03\end{array}$ & $\begin{array}{l}3.9 E-05 \\
2.3 E-04 \\
4.7 E-04 \\
1.4 E-04 \\
5.5 E-03 \\
1.6 E-07 \\
1.1 E-07 \\
\text {.1E-OS } \\
3.0 E-07 \\
4.1 E-04\end{array}$ & $\begin{array}{l}-05 \\
-04 \\
-04 \\
-06 \\
-03 \\
-03 \\
-00 \\
-09 \\
-05 \\
-10 \\
-04 \\
-04\end{array}$ \\
\hline 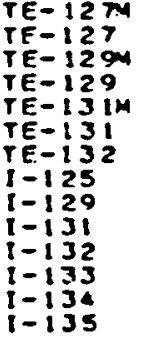 & 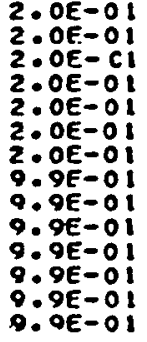 & $\begin{array}{l}1.0 E-04 \\
1.6 E-05 \\
5.0 E-04 \\
6.0 E-06 \\
2.8 E-03 \\
8.9 E-06 \\
6.0 E=03 \\
1.1 E-0.04 \\
5.1 E-04 \\
1.5 E-04 \\
8.9 E-05 \\
1.0 E-04 \\
1.3 E-05 \\
1.6 E-04\end{array}$ & $\begin{array}{l}3.7 E-04 \\
1.3 E-05 \\
6.2 E-0 \\
7.2 E-06 \\
6.1 E-04 \\
2.3 E-05 \\
1.1 E-03 \\
1.2 E-04 \\
5.2 E-04 \\
2.1 E-04 \\
2.8 E-04 \\
2.1 E-04 \\
2.04-04 \\
3.2 E-04\end{array}$ & $\begin{array}{l}0 E-04 \\
2 E-05 \\
3 E-04 \\
3 E=06 \\
8 E=04 \\
9 E=06 \\
5 E=04 \\
2 E=04 \\
6 E=04 \\
2 E=04 \\
6 E=05 \\
9 E=04 \\
2 E-05 \\
0 E=04\end{array}$ \\
\hline
\end{tabular}

\begin{tabular}{|c|c|c|c|}
\hline 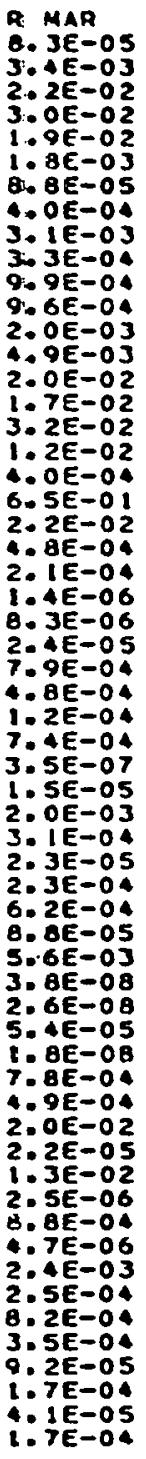 & 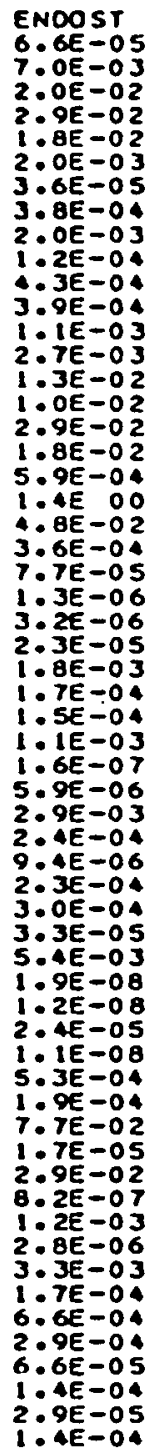 & 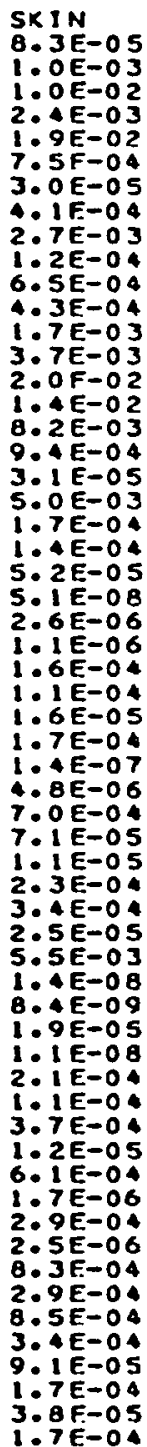 & 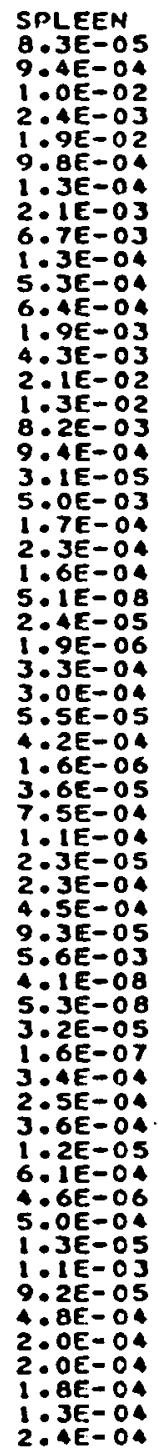 \\
\hline
\end{tabular}

TESTES
B.3E-OO $7.2 E-04$

$2.4 E-03$

$1.9 E-02$

$2.9 \varepsilon-05$

2. $6 E-03$

2: $4 E-04$

5. $5 \varepsilon-04$

$1.5 E-03$

2. OE-O2
$: 0 E-02$

$1: 1 E-02$

政.

3. $1 E-05$

5. $0 E-03$

3 SE 04

(1E-0

$1: 2 E=06$

$1.5 E-06$

$1.9 E-04$

S. $2 E-05$

5. $3 E-08$

2. $8 E-06$

$1.0 E-04$

$1.2 E-05$

$2.3 E-$

2. $\theta E-05$

: $3 E-08$

$1: 0 \mathrm{E}-0 \mathrm{~B}$

2. $8 E-05$

3. 0 : 04

$2.2 E-04$

: $2 E-05$

$6: 1 E=04$

1. $5 E=06$

$1: 6 E-06$

i. IE-O

5: OOE-OA

1. SE-O4

$1.3 E-04$
$2 . S E-05$

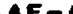

9. $6 \mathrm{E}-$

$2 . A E=0 J$

$6.2 E-0$

$1.7 E-05$

2. $3 E-03$

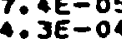

$2.6 E=04$

3:A $A E-03$

$1: T E-02$

Q. $3 E-03$

3. 1 E- OS

5. OE 03

1.TE-:

5. $9 E=08$
9. $9 E=07$

5. $9 E=07$

2. $a E=05$

$7.1 E-06$

$6.2 E-05$
$9.5 E-O B$

2.11E-06

4 SE-OS

7. TE

2. $3 E=04$

5.5E 1 - 03

1. $2 E=08$

5.2E-09

$1.2 E=05$
$6.5 E=09$

6.SE

$3.62-0$.

S. $1.2 E-0.04$

5. $9 E=04$

$1.9 E-0.06$

3. $5 E-06$

$4.5 E=04$

$1.1 E-03$

9. $3 E-05$

2. $8 E=04$
B.JE-OS

$1.1 E-02$ B. $6 E-03$

$2: A E-03 \quad 2 . A E-03$

1: $:$ BE-OS

$\therefore O E-O 5 \quad 2.2 E-04$

3. OE-O3 3. $8 E-03$

$3.2 E-04$
$3.5 E$

$3.2 \varepsilon=04 \quad$ I:ZE-0

$1 . B E-03 \quad 2.4 E-03$

$\begin{array}{ll}4.0 E-03 & 6.6 E-03 \\ 2.4 E-02 & 2.0 E-02\end{array}$

$1.7 E-02 \quad 1.2 E-02$

9.4E-04 9.4E-04

$3.1 E-05 \quad 3.2 E-05$

S.0E-03 $5.0 E-03$

$1: 1 E-04$ TAE-04

$5.1 E-08$ 5.TE

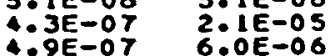

3.:E 205 6.0E-06

$1.0 E-05$
$6.6 E-06$

5.0E-OS $1.2 E-03$

3.1E-OB

7.9E-0?

3. $9 E-05$

3. $2 E-04$

3. $0 E=03$

$7.6 \mathrm{E}-06$

$5.0=03$

i:se-O9

$1.2 E-05$

1. $3 E-04$

$1: 9 E-05$
$3: 7 E-04$

1.: $2 E-05$

6.:0E-04

$3.96-02$

5. $3 E=03$

$1.3 E$ :

I:TE OO

i. AE- 02

$1.7 E=01$
$2.2 E=01$

: $7 E-04$

2. $3 E-05$

$9.3 E-04$

$2.1 E-04$

6.0E-08

$3.8 E-08$

$9.6 E-05$

$1.8 E-08$

9.6E-O4

3. $8 E-04$

1.AE-OS

$6.8 E-04$

$4.8 E-06$

$1: 3 E=3$

$7.7 E-06$

1.0E-0.

SSE-OA

$8.6 E=05$

a
$1: 0 E-05$
$1.5 E-04$ 
INGESTION DOSES AT SO YEALS (REM MICROCURIE)

\begin{tabular}{|c|c|c|}
\hline 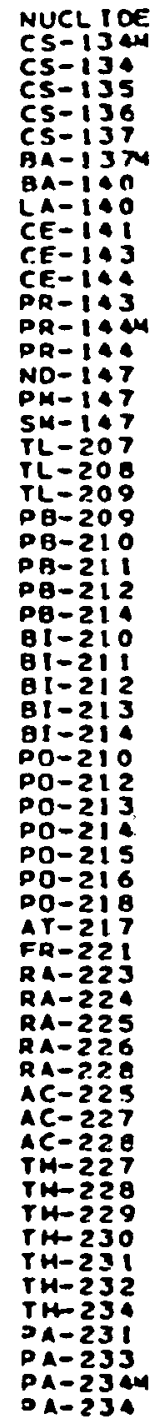 & 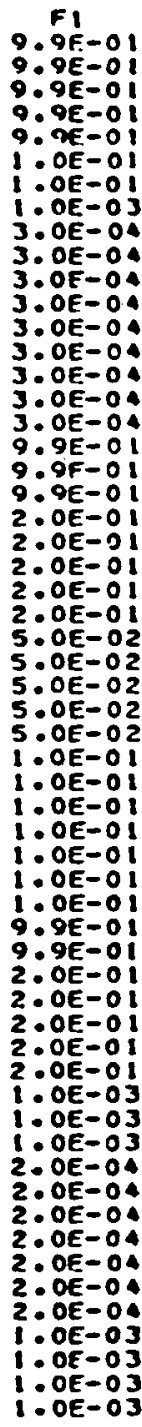 & 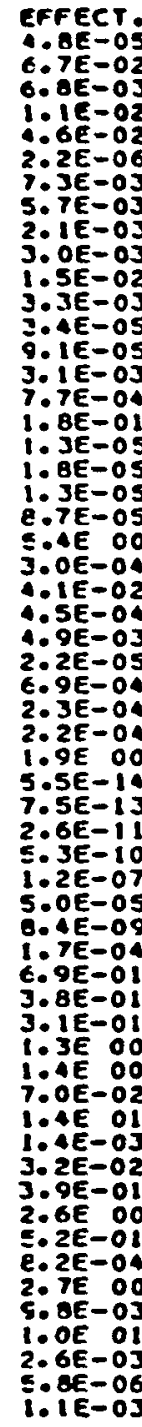 \\
\hline
\end{tabular}

LUNGS

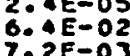
$?: 2 E-03$ 4 TE-02 $2: 2 E=0:$

5. $3 E-06$

2.AE-05

$2.8 E-O B$
$7.6 \mathrm{E}-0 \mathrm{~B}$

6. $3 E-09$

:.2E-06

$2.7 E-06$

$1.6 E-06$

5. $8 E-01$

6. $\theta E-03$

9. $7 E-05$

3. $9 E-08$

$1.7 E-05$

$9.1 E-06$

3. $\begin{aligned} & 1 E-01 \\ & 3.2 E-34\end{aligned}$

$4 . \theta E-16$

2. $6 E-11$

$6.1 E-06$

$2.0 E-09$

i:OE-OA

8. TE 02

$8.7 E-02$
$3.0 E=01$

5. 9E-: I

$8.0 E-04$
$2.7 E-05$

2

8. $9 E=03$

2. $6 E-03$

$5.3 E=07$
$4.0 E-03$

2. $A E=06$

$1.4 E-05$

S. $2 E-09$
S. 6.6E 30.02 $1.2 E-02$ 3. . $1 E-0$ :OE -OJ 2. $1 E-03$

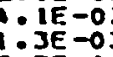
$\begin{array}{rl}1.3 E & -03 \\ 5 & 7 E-0.4\end{array}$ SE $5 E-03$ $2: B E-04$ $1.9 E-O J$ . SE -OA 7 OE -04 $3.0 E-01$
$30-03$ 1. $1 E-02$ I. TE -0J 5. $9 E-03$ $2.9 E-03$ 3. $2 E-01$ $92 E-13$ : : $3 E-10$ $8.3 E=09$ 6. $9 E-04$ IE-O $10 E-03$ . OE -OI 1.06
$3 \cdot 3 E-0.01$
$3 E-01$ $1: 9 \varepsilon-02$ 7.0E 0.04 $6.1 E=03$ $1 . A E-02$ $6.6 \varepsilon-03$ 7.2E-0. 3:: $: E=03$ 证 $9.6 E-05$
$2.9 E-0$.

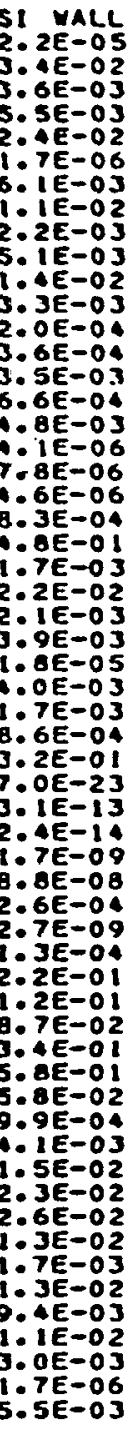

ULI YALL

$3: 8 E-02$
$3: 6 E-03$

$3: 9 E-03$
$3: 0 E-02$
10

3: $0 E-02$

1.2E-06 $4.3 E-07$

3. $4 E-02$ 6.5 F.02

$2.1 E-02 \quad 3.2 E-02$

Q.5E-O1

$\begin{array}{ll}1: 9 E-02 & 5.4 F-02 \\ 3 E-05 & 2: 5 E-06\end{array}$

$6.6 E-05 \quad 3.6 E-06$

3. $9 E-03 \quad 1: 2 E-02$

$\begin{array}{ll}2 . \theta E-02 & 8.5 E-02 \\ 2: \theta E-07 & 7.1 E=0 A\end{array}$

$5.4 E-06 \quad 2.2 E-06$

$1: \bar{B}-03 \quad 53$

$\begin{array}{lll}4.8 E-01 & 1.9 E-01\end{array}$

$6.3 E-02 \quad 7: 4 E-02$

$9.3 E-04 \quad 1.9 E-04$

G:SE-07 $406 \mathrm{E}-08$

$2.4 E-03 \quad \because 7 \mathrm{~F}-04$

$9.2 E-O 4$
$2: 2 E-04$
3

3.7E-0I $\because 9 E-01$

$\begin{array}{ll}1: 1 E-33 & 3.2 E-34 \\ 5: 0 E-13 & 2.5 E E-13\end{array}$

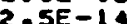

$1.6 E-10$
$5.6 E-07$

1.8E-OS

$1: 1 E-04 \quad \because \because 0 E-04$

$3.1 E-01$ T

1.OE-O I $18 B E-01$

$6.0 E=01 \quad 6.6 E-O 1$

3. $1 E-01$

$5.0 E-03$
$8.9 E-03$

$8.9 E-03$

$1: 1 E-01$

6. $1 E-02$

$7.0 E-03$
$5.5 E-0.25$

S.
S. $A E-0.02$

$6.5 E-02$
$1.3 E-02$

B.
$1.1 E-0 B$

$8.7 E-0$

$2.6 E-02$
$3.0 E-03$

$3.4 E-0$

2. $3 E-01$

$1.8 \mathrm{e}-01$

: $6 E-01$

2.0E-OI

3.8E-02

$1.6 E-08$
$9.1 E-03$
ADRENAL B.7E-O5 $1: 3 E-02$ 5. (

$7 E-05$
$.4 E-05$ 3. $3 E-05$ 1. ( $2.8 E-05$

.2E-06

(1)

2. $6 E-06$

. $8 E-01$

5. $8 E-05$

$6.9 E-03$

\begin{tabular}{l}
$1.0 E-05$ \\
\hline
\end{tabular}

O9E-0B

$3.4 E-05$

-4E-05

$3.1 E-02$

$3.2 E-34$

$2.4 E-14$

.2E-0B

6.8E-06

(6.)

TE-O

.7E-02

$3.3 E-01$
$5.9 E-01$

.6E-04

:AE-03

5. 0 E-OA

B.

$2.6 E-03$

$5: A E-06$

$5.5 E-06$

.9E-0

$5.9 E-09$
$1.4 E-04$
BL YALLL

8. $1 E-02$

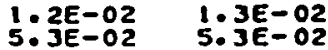

$1.6 E-07 \quad 8.6 E-07$

$17,03 \quad 8.2 E=04$

$1.2 E-04 \quad 4.4 E-05$

2.1E-04 $1.0 E-04$

2. QE-OB 1.SE-OS

$1.6 E-07$

1. $8 E-04$ 7. $1 E-05$

$1.3 E-0 B$

i: 2E=06

$9.0 E-07$

2: $3 E-06$

5. $8 E-01$

7.1E-03

$3.6 E-00$

5. $0 E-05$

3.: OE-DS

3 3E-01

3. $2 E-34$

$2: 4 E-14$

$2: 3 E-O B$

:

I:OE-OA

Q. $\mathrm{aE}-02$

8. $3 E-02$

5: $9 E-01$
$2.6 E-03$
$1.9 E$

1 $9 E=04$

$6.6 E-04$

9: $1 E-03$

2. $6 E-03$

ㄱ. $7 E-03$

2. $1 E-04$

2. $\theta E-04$

3: $3 E=04$

$8.7 E-09$
$4.2 E-06$

8. $6 E-07$

$5.2 E=06$
$4.3 E-06$

$1.0 E$ OI

2. $1 E-05$

7. $6 E=02$

$2.2 E-02$

T. IE-03

$1.5 E=04$
$2.3 E-04$
$3.6 E-30$

$9.4 E$. 00

3. $6 E-38$

$7 \cdot 3 E-13$

3. $3 E-11$

1. $2 E-05$

$6.2 E-10$

6. $8 E-06$

6. $3 E-02$

8. IE 02

6.0E-01

9. BE- 04

1: $1 E-0.04$

B. $9 \mathrm{E}-\mathrm{OJ}$

$1.4 E-02$

$2.6 E-03$

$\therefore$ TE=06

.

1.2E-05

$1.3 E=04$

B. $8 E=09$
LI VER

7. $A E-02$
$7.2 E-03$

$1.2 E-02$

5.1F-02

$9 E-04$

7. 2E-05

Q. IE-0S

3. $1 E-05$

i. TE-O

$7.0 E-05$

2. 6E-04

$1.3 E-07$

$3: 2 E=06$
$1.7 E=06$

. AE-06

$4.2 E-05$

1: $3 E-04$

3. $B E-D B$

3. $0 E-05$

$1.0 E-05$

5. $0 E-37$

6. $6 E-16$

$8: 1 E-12$

$6.1 E-08$
$3.4 E-06$

$4.6 E-10$

S. JiE-01

8. $5 E-02$

3. $3 E-01$
5. $B E-01$

T. 1E-O2

$\begin{array}{rl}5.6 E & 01 \\ 3 & 0 E-03\end{array}$

3. $9 E-03$

1. IE-O 1

$3.6 E-06$

3. $B E-02$

$1.6 E-02$

- $9 E-05$

2. $2 E=04$

PREAST

T:AEE-:

$1.2 E-02$

5. $1 E-02$

DE-D

:GE-0.

$8.6 E-05$
$\therefore .5 E-05$

OE-OB

作

6. $9 E-05$

$8.3 E=06$
$4.2 E-007$

1. $3 E-07$

: $3 E-06$

S. BE-OI

600

$1.0 E-0.04$
$7.5 E-05$

$3.4 E-O$
$4.6 E-O S$ 
I NGESTIOA DOSES AT 50 YEARS (REM/MICROCURIE)

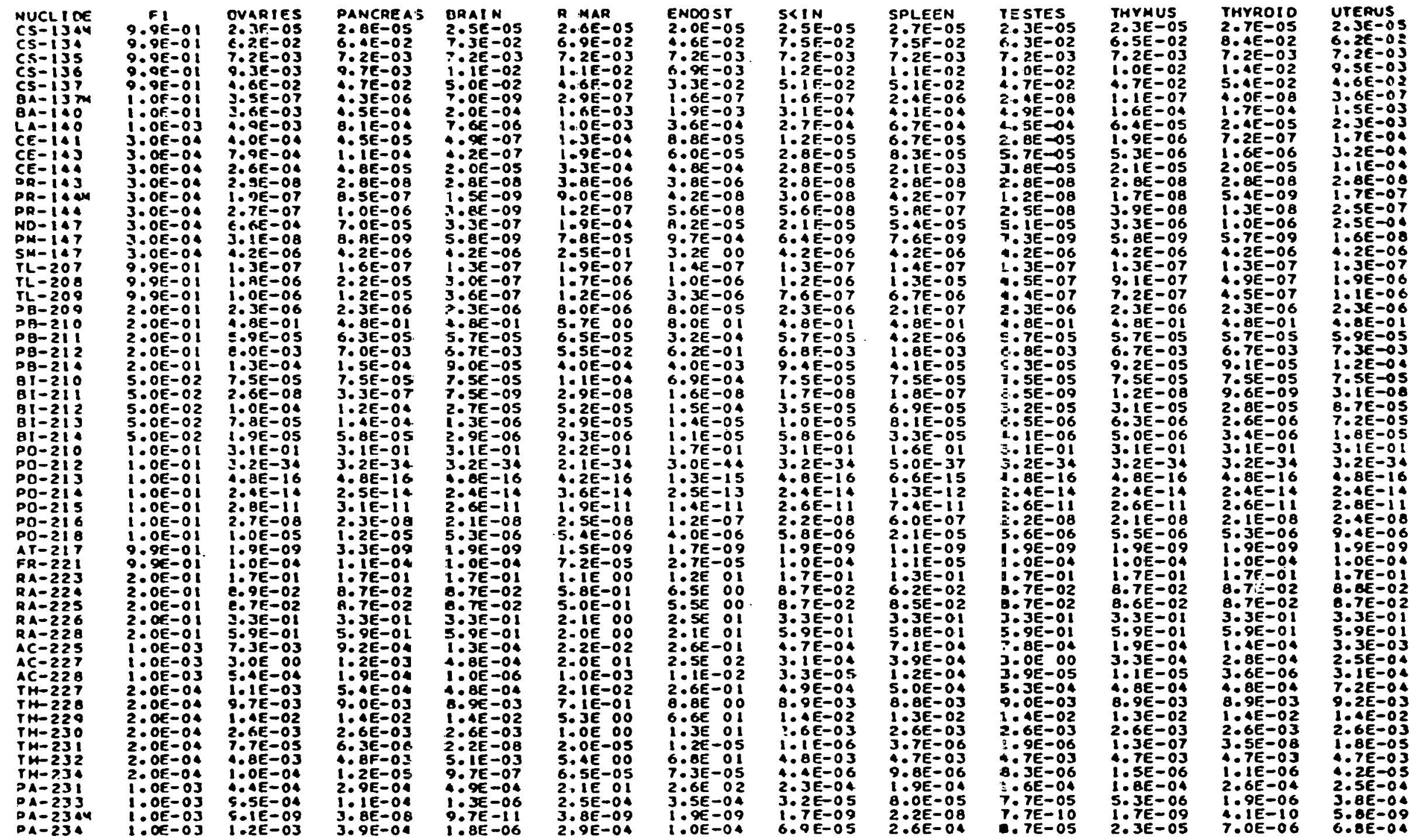


INGESTION OOSES AT $5 O$ YEARS (REMPHÁCROCURIE)

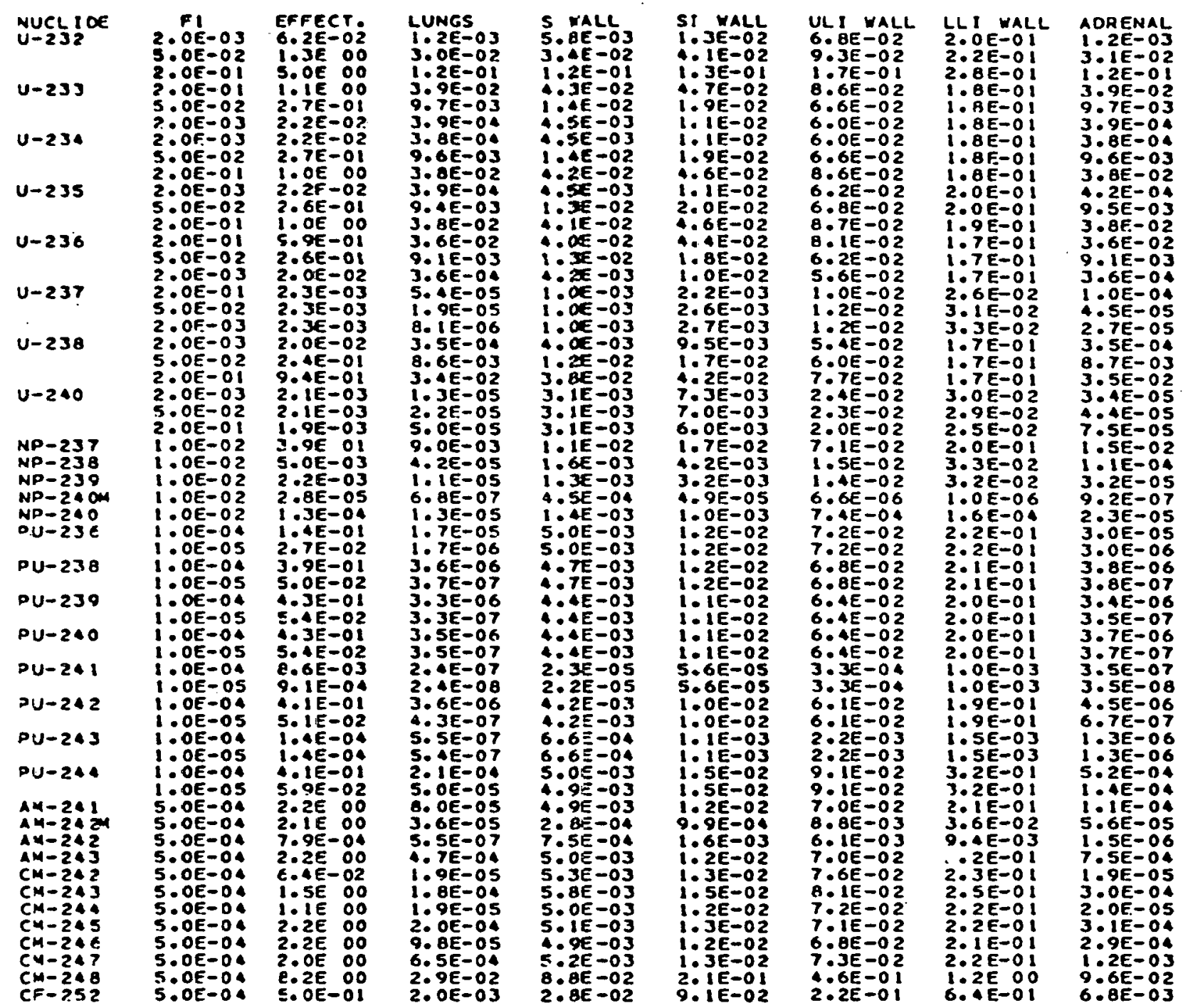

1. $2 E-03$ 3:2e-01

3: $9 \in-02$

3: $9 \mathrm{E}-\mathrm{O}$

3. $8 E-04$

$3: a \varepsilon-02$

$6.2 E-04$

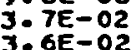

- $1 E-03$

$3.6 E-04$
$2.0 E-04$

$1.9 \varepsilon-04$

: $6 E=04$

B. $6 E-03$

3. $4 E-02$

$1: 1 E-04$

$2.3 E-03$

3. $9 E-04$

$1.7 E-04$

$3.6 E-07$

7. $9 E-06$

$6.61 E-07$

3. $3 E-06$

S: $\begin{array}{ll}0 E-07 \\ \text { AE } 06\end{array}$

. OE-O

5. $\theta \varepsilon-09$

5. $1 E-06$

2. $3 E-06$

3. $A E-06$

列

6. $T E=05$

$7 E-06$

$2.9 E-04$

$2: 5 E=04$

$1: 9 E-05$
$2: 0 E-04$

$1.6 E-04$

5. $0 E-04$

S.
$1: B E-02$
$B E-02$

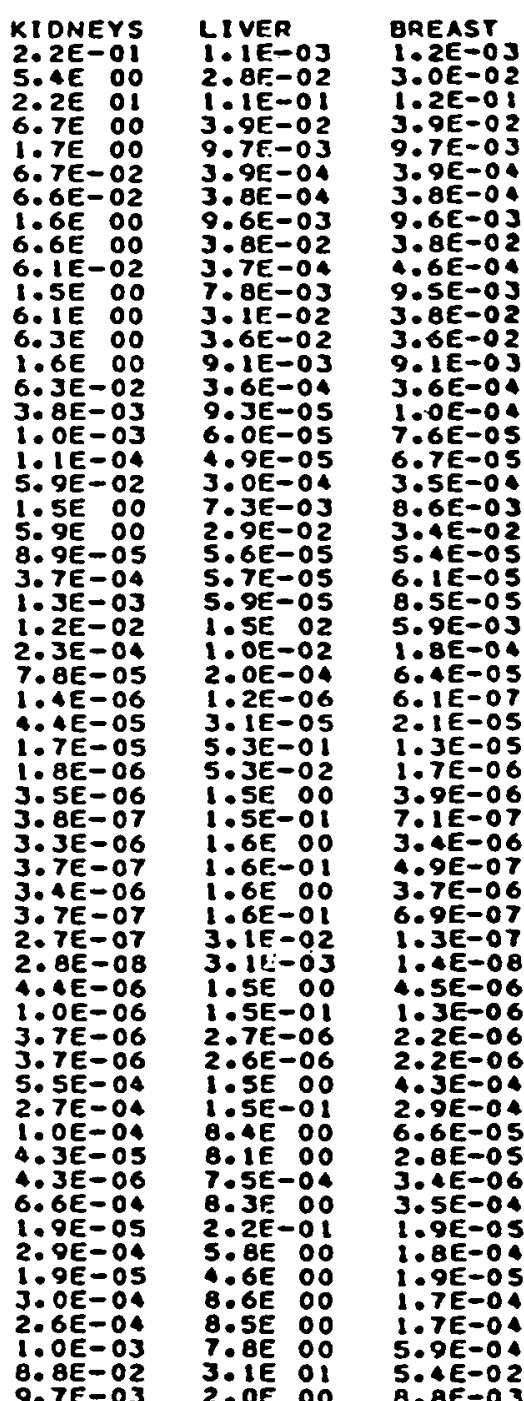


INGESTION DOSES AT 50 VEARS (AÊAMACROCURIE)

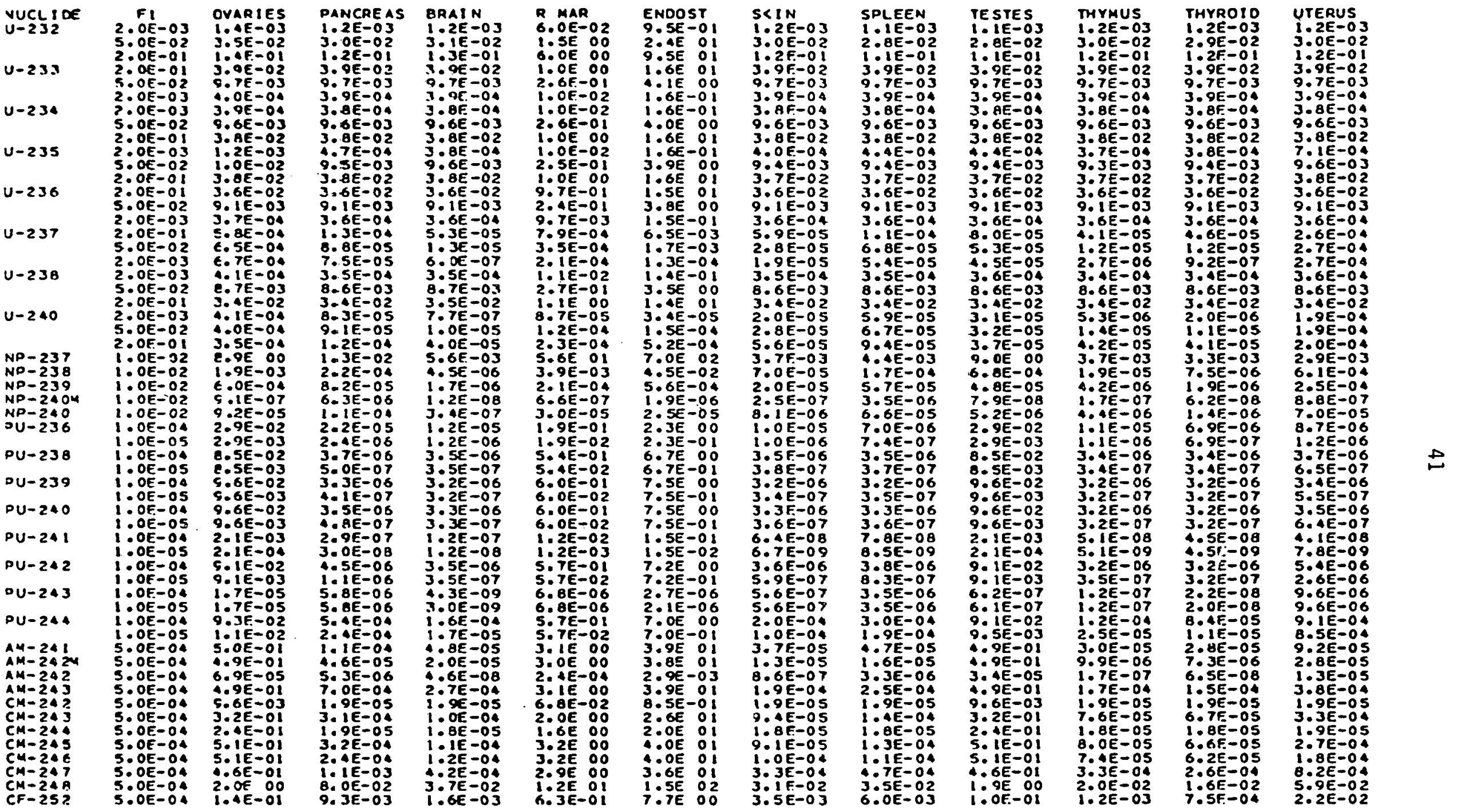




\section{APPENDIX II}

ESTIMATES OF 1-YEAR DOSE EQUIVALENT

FROM ACUTE RADIONUCLIDE EXPOSURES 
INHALAT:ON DOSFS AT I VEAR

(REM/MICROCURIE)

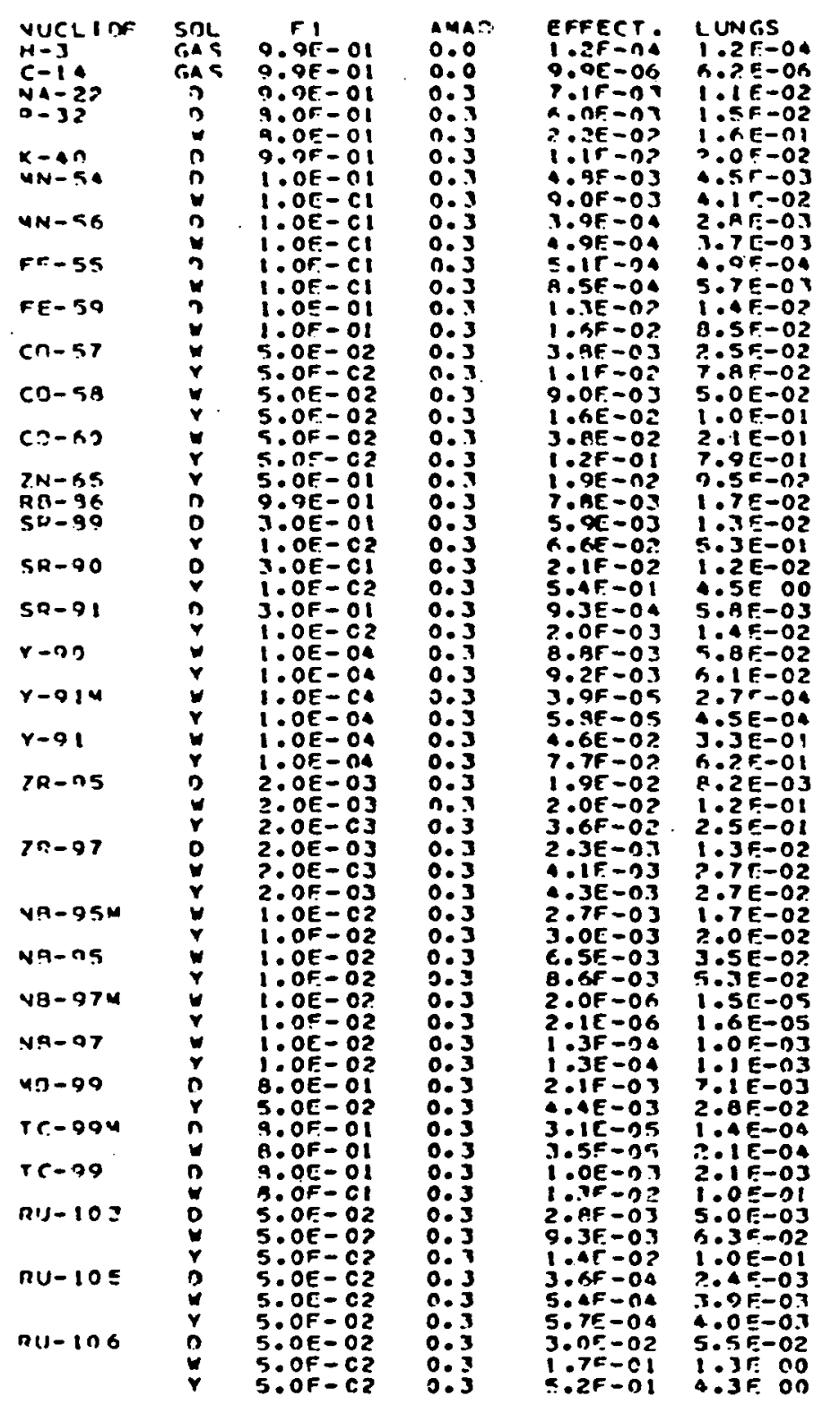

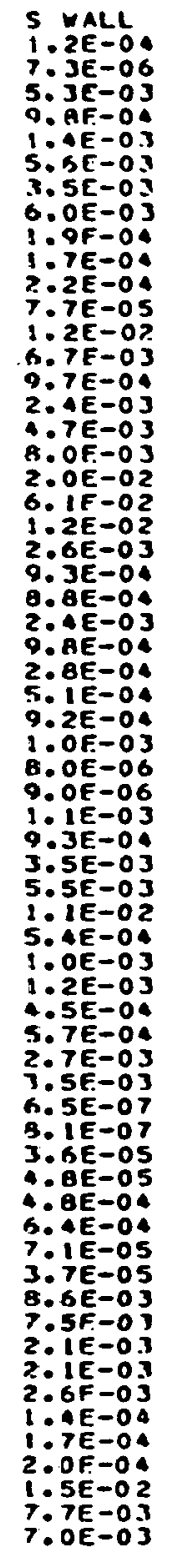

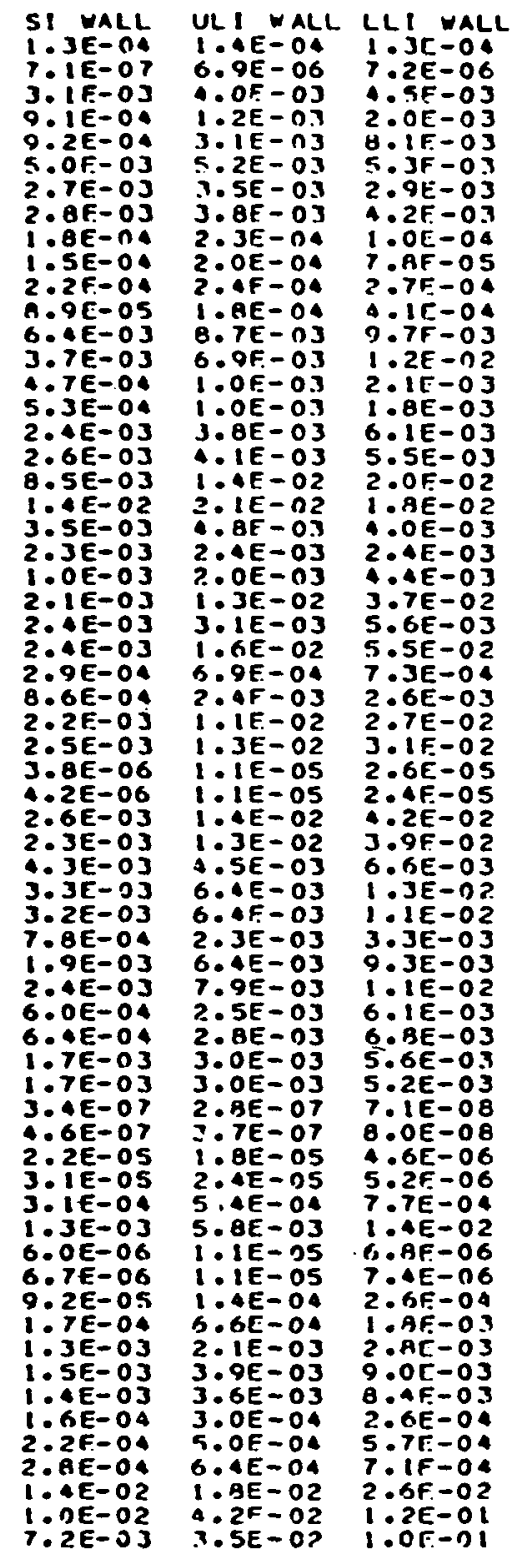

ADRENAL BL FALL

KIONEYS T.AE-OS $5.3 E-03$ O. 1.DE-02 $1.0 E-02$ $6.6 E-03 \quad 2.5 E-0.3$ 7.9E-03 $1.3 E-03$ 5.16-OS 1.3E- OS T. - OA TE-O

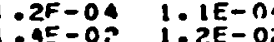
$7 . x-0 j$

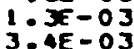
5. $A E-03$ OA $6 . x E=02$ $1.55-02$ GE-O $2: 2 E=05$
$4.7 E=03$ $1.5 E-04$ $2.0 E-0:$

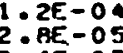
. $6 E-07$ $6.7 E=06$ O. IE-05 $3: 1 E-0$
$3 E-03$ $3 F-0$
$3 F$ $5: 1 E-04$
$3: 0 E-04$ उ.OE-OA $3 . A E=04$
$3 . A E=04$ $3.2 E-05$

$4.11 E-03$
$3.5 E-007$

$3.4 E-07$

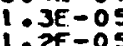

$6.3 E-04$

$1: \frac{B F=04}{0}=05$

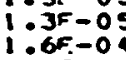

$1: A F=04$

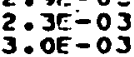

5. $9 \overline{Q i}-02$ 5. $4 F-05$

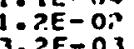
2. $7 E-04$ $1.1 E-03$ (a)

4. $\mathrm{BE}-03$

. $6 E-03$

$1.6 \mathrm{E}-0.3$ $2: 1 E-05$
$4: 7 E-0.34$

I $\triangle E=04$ 3. $9 E-05$

$2.8 E-05$
$9.6 E-07$

$8.6 E-07$

3. $1 E-04$

1.7E-05

1.2E-03

3. BE-04

$1.2 E-04$

?: YE-0S

$6.5 E-04$
$3.6 E-04$

3. $\triangle E E-O Q$

1.8E-0

B. TE-OT

A. SE- $A 4$
$8 E-05$

$9 E-06$

$1.6 E-04$

I:A $A E=04$

7. $6 E-04$

5. $1 E-05$

T. IE-OS

2. AE 02 S.AE-OJ I:ZE-OJ

OIE-OA

$6.5 E-0.3$

$9.2 E-04$

S. $1 E-03$

S:1E-03

3. $3 E-05$

$1.1 E-04$

$5.2 E-03$
$1.4 E$

$1.5 E-04$
$11 E-03$

2. $\mathrm{AE}-03$

$1.3 E-02$

$3.4 E-02$
$8.3 E-03$

$0.6 E-03$
$1.6 E-03$

$2,1 E-05$

$1: 5 E-04$

$7.4 E-05$

$9.6 E-07$

3. $1 E-06$

$2.8 E-05$
$5.8 E-05$

$3.6 E-03$

उ.JE 30.04

$2: 1 E-0$
30

3.2E 204

$2.1 E-03$

$1.9 E-03$
$1.6 E-07$
$.5 E-07$

$6: 3 E-06$

$5.6 E-06$

$2.5 E-04$

$7.4 E-06$
$5.8 E-06$

$1.6 E-04$

$1: A E=04$
$2: \theta E-03$

$1: 3 E-03$

6:3E-05

2.0 $0 E-0 S$

$2.8 E-02$
$7.8 E-03$
$3.6 E-03$

LIVER BREAST 1.2E-O. TE-03 $6.15-03$ D TE-0J $1.7 E-03$ $1.0 E-02 \quad 1.0 E-02$

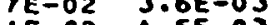
IE-02 5 SE- J - IE-OS 3:BE-OS 作 .4E-02 1.2E-02 DE-03 $5.2 E-03$

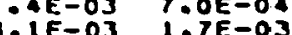
. aE-0J $3: J E-03$ 5E-03 5.5E-03

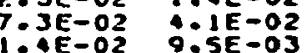
$4.6 \mathrm{E}-03$ O.6E-03 $.6 \mathrm{~F}-03$ $1.2 E-05$
$4.6 E-03$
$.5 E-04$ $.5 E-04$ 1.SE-OA l. 3E-04 7.9E-0.5 2.8E-0.5 .5E-05 $\triangle .0 E-06$ 年 $? 3 E-04$ $3: O E-O 6$
$3 \mathrm{~B}-06$

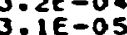
. $3 E-02 \quad 7.4 E-03$ C.TE 2E-04 2.0E-0. $\begin{array}{ll}.3 E-04 & 2.1 E=04 \\ 3 E-04 & \end{array}$ 3.0E-0J $1.9 E-03$ $3: 1 E-03 \quad 2.4 E-03$
$3.5 E-07$ .4E-07 $1.9 E-07$ .2E 205 7.7E-06 $6.3 E-03 \quad \because 8 E-04$ .2E-0S $8.2 E-04$ $202 E-2 E-0 E$ OAE-OA $1 . A E-O A 4$ .3E-03 1.5E-03 $3: 0 E-03 \quad 1.7 E-03$ . $9 E-05$ 3. $0 E-05$ . $8 E-02 \quad 2.9 E-02$ $\begin{array}{ll}9.6 E-03 & 7: 9 E-03 \\ 6.4 E-03 & 4.2 E-03\end{array}$ 
INHALATION DOSES AT I YEAR

(REMPMICROCURIE)

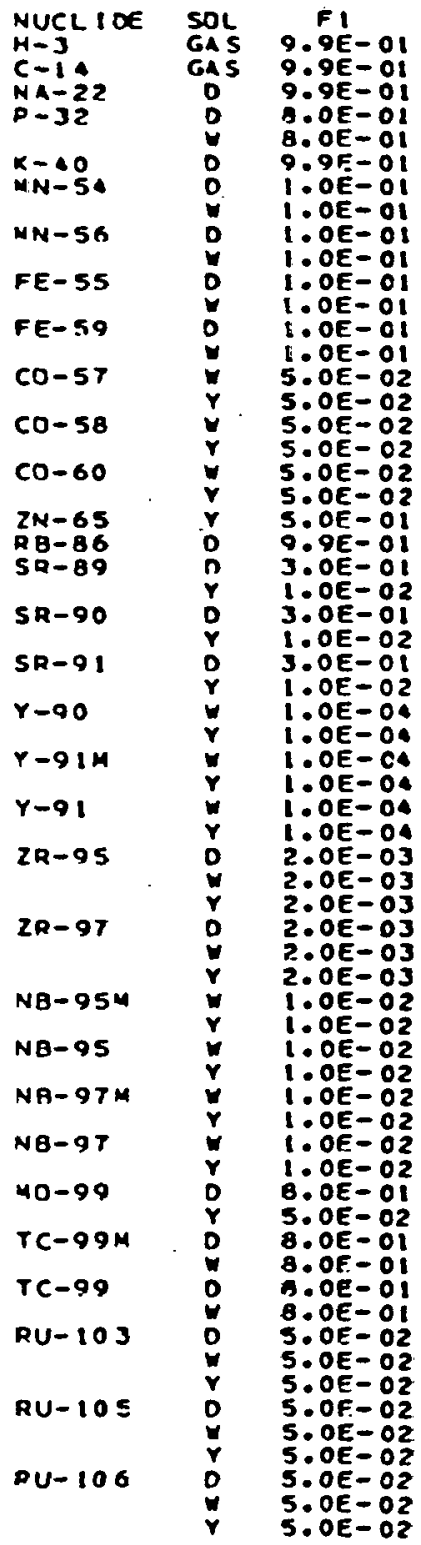
0.3 0.3 0.3 0.3

AMAO

ovaries

PANCREAS GRAIN

R MAR ENDOS $\begin{array}{lllll}5.2 E-0 . & 1.2 E-04 & 1.2 E-05 & 1.2 E-04 & 9.8 E-05 \\ 5.3 E-06 & 7.8 E-06 & 5.0 E-06 & 2.4 E-05 & 5.1 E-05\end{array}$

$\begin{array}{llllll}0.3 & 5.0 E-03 & 6.0 E-03 & 6.6 E-03 & 1.1 E-02 & 9.9 E-03 \\ 0.3 & 1 . T E-03 & 1.7 E-03 & 1.7 E-03 & 2.1 E-02 & 2.0 E-02\end{array}$ $\begin{array}{ll}1.7 E-03 & 1.7 E-03 \\ 0.2 E-0.04 & 9.2 \mathrm{E}-04\end{array}$ $0.3 \quad 2.8 E-03 \quad 1.0 E-02$ $3.5 E-03$ $\begin{array}{ll}.0 E-05 \\ .2 E-05 & 7.3 E-05 \\ 3.2 E-05 & 05\end{array}$

$6.3 E-05$

$1.7 E-03$ $1.0 E-02$
$3.8 E-03$ $\because .9 E-0 J$ $1 \mathrm{IE}-0$

$1.7 E-03$
$3.4 E-05$ Q. $6 E=03$ $9.2 E-04$
$1.0 E-02$

TESTES 1. ZE $=04$ S. 4 E- 06

TMYMUS 0.3 3.BE-O3 T.1E-O $0.3 \quad 3.5 E-04 \quad 3.3 E-03$ $2.1 E-03 \quad 5.5 E-03$ $\begin{array}{ll}7.0 E-03 & 1.0 E-02 \\ 9.5 E-03 & 2.2 E-02\end{array}$ $9.3 E-03 \quad 7.1 E-02$ $3.6 E-03 \quad 1.3 E-02$ $1.6 F-031.6 E-03$ $\begin{array}{ll}2.1 E-05 & 2.2 E-05 \\ 4.7 E-03 & 1.7 E-03\end{array}$ $1.3 E-041.5 E-04$ I. $3 E-04$ $2.8 E-05$
$9.6 E-07$ $1.2 E-06 \quad 7.9 E-06$ 3.2E-04 3.3E-04 2.1E-05 $4.2 E-05$ .TE-03 6.8E-03 $2.1 E-03$ 1.AE-02 $3.9 E-04$
$3.7 E-04$
$.3 E-04$ -3E-04 3.6E-04 $1.8 E-0$. I. $5 E-03$ 3.2E-O $\begin{array}{ll}1.3 E-03 & 4.3 E-03\end{array}$ $\neg .1 E-0$ $3.2 E-06 \quad 1.4 E-05$ $4.4 E=045.0 E=04$ 2.4E-O4 2.0E-0 $4.9 E-06$ $3.5 E-06$ 1.5E-OS $1.6 E=04$ I.AE
$2.4 E-04$
$1.4 E-03$ 1.2E-03 $1.6 \mathrm{E}-04$ $1.4 E-5 E-03$
3.6 $3.4 E-03$

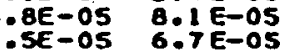
$0.3 \quad 5.8 E=05$

1. उE-OS

4 iE-OS

$4.9 E-05$

$2.6 \mathrm{E}-03$

1. TE-03

$1.7 \mathrm{E}-03$

1. OE $\mathrm{OE}-02$

$9.2 E=0$

$7.1 E-06$
$5 . A E=03$

THYROTO

I.2E-O $3.1 E-05$ 2.3E-O

$6.4 E-03$ . $1 E=03$ . $9.8 E-03$ 1. IE-02 $6.5 \mathrm{E}-03$ $1.7 E-03$ $1.0 E-02$ J.

OAE-05 $9: 1 E-05$ M:OE-05

3. $7 E-0$ $\begin{array}{ll}3.6 E-04 & 3.0 E-04 \\ 1: 1 E-04 & 1.2 E-04\end{array}$

$2.0 E-03$

i. IE- IE $\because 1 E-02 \quad 7.9 E-03$ $2.0 \mathrm{E}-0.0$

$1.1 E-03$

(1)

. $0 E-0$ $3.8 E-0$. IIE-OZ $1.9 \mathrm{E}-03$

$5.5 E-03$

.4E-04 2.6E-03 2.0E-03 $6.8 E-0$

$3.2 E-03 \quad 2.3 E-03 \quad 1.9 E-03$

. $9 E-03$ 5. $6 E-05$

5. $8 E-04$ 9

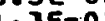

.0E-0J 2.9E-03 B:BE-0

3. $8 E-02$

$0.7 E-03$
$2.7 E-03$

$1.96-02$

3. $2 E-04$

$1.6 E-02$

(1.5e-03 6.8E-0

$1.6 E-03 \quad 1.9 E-02 \quad 2.7 E-02$

1. OE

$1: 3 E-01$

$1.4 E-02$

5. $T E=0.3$

$1.2 E-03$
$3.2 E-03$

S.

$3.6 E-02$

$4.5 E-03$. $46 E-03$ a $7 E-0$

I. $K E-03$

$2: 4 E-04$
$9: B E-02$
3

$2.1 E=05$

$47,75-03$

$3.0 E E-03$

$1.5 E-03$
952

$4.7 E-03$

$2: Z E=05$

$1.6 E-03$

$1.6 \mathrm{E}-03$

$2 \cdot 2 E-05$
$4.7 E-03$

P. $5 E=0$.

$1: 5 E-04$

$1.5 E-04$

5. $9 E-05 \quad 3.9 E-05$

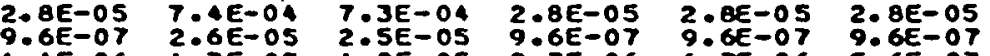

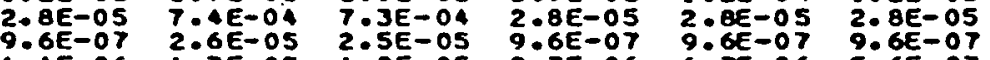

$1.5 E-04$
$2.2 E-04$

$1.66-03$

$2: 2 E=05$

i: $3 E-\infty$

$6.1 E-05$

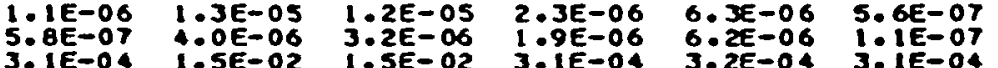

$3.9 E-06$

$2: 8 E-05$
$: 6 E-07$

$2.8 E-05$
$9.6 \mathrm{E}-07$

.OE-05 3.3E-06

7.2E $2.3 E-05$ 3.9E-05 $1.5 E=05$

$3.4 E-04$

$6.0 E-03$

8. $7 E-O$

$3.8 E-03$

$1.2 E-02$

3. $1 E-04$

$3.7 E-03$ 3.OE-O

$3.6 E-04$
$3.1 E-04$

. BE-04 $1: B E-03$

$\begin{array}{lll}7.6 E-05 \\ 2.7 E-05\end{array}-4 E-04$

$3.7 E-04$

$1.4 E=-04$
$9.9 E=-O 5$

(

2.1E-02

$3.2 E-0$.

A. $8 E-03$

$3: 9 E-03$
$7: 1 E=03$

$4.2 E-04$

(1)

$1.8 E-04$
$2.8 E-05$

$5.1 E-041.0 E-03 \quad 1.2 E-04$

(

2. $8 E-05$

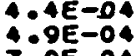

$1,9 E-04$

$1: B E=04$

$4.6 E-03 \quad 1.8 E-03$

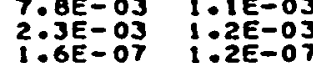

$3: B E=03$

2. $9 E-04$

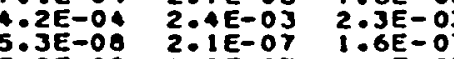

90

$3.2 E-07$
$3.2 E-0.7$

S. $B E-00$

6.7E-03

$5.5 E-07$

$2.2 E-03$
20

$\begin{array}{ll}: 1 E-05 & 1 \cdot 3 E-06 \\ 1: 1 E-05 & 2.5 E=07\end{array}$

$\begin{array}{lll}1.9 E-06 & 4: 4 E-06 & 1: 1 E-05\end{array}$

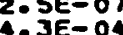

$\because$
$3 \mathrm{~B} E-04$

$6: A E-06$

$19 E-03$

$4.6 E-04$

$1.7 \in-0$

3. $1 E=05$

$49 E-06 \quad$ I:IE-05 $6: 7 E-06 \quad 5: 7 E-06$

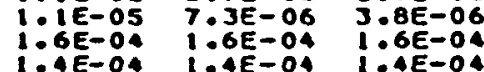

I:ZE

. $6 E-0$

$1.66 \mathrm{E}=0$.

i. $6 E-06$

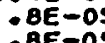

$1.8 \mathrm{E}-07$

(

i-IE-O4

$1.5 E=05$

(1)

$1: 4 E-: 03$
$7 E-03$

$1.4 E-D 4$

-0.03
$1.11 E-03$

$0.3 B$
$1.0 E-0.04$

2. $1 E=03$

S. $2 E=04$

$1.6 E-04$

:TE-05 6:4E-05 5:1E-05 5.4E-OS

$\begin{array}{llll}1.3 E-05 & 3.9 E-05 & 2.9 E-05 & 2.3 E-05 \\ 5.1 E-06 & 3.5 E-05 & 2.4 E-05 & 1.6 E-05\end{array}$

$\begin{array}{ll}2.7 E-02 & 2.7 E-02 \\ 7.4 E-03 & 8.7 E-03\end{array}$

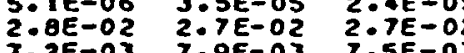

$\because: 9 E-03$
$: 1 E=03$

$3.5 E-03$

$\begin{array}{ll}2.8 E-02 & 2: 8 E-02 \\ 7: 5 E-03 & 8: 5 E-03 \\ 2: 9 E-03 & 5.9 E-03\end{array}$

A.AE-05

1.OE-OS

: : $3 E-04$

$5.0 E-05$

$3: 7 E-03$

$4.4 E-03$
$0.1 E-05$

$2.6 E=03$

1.5E-03

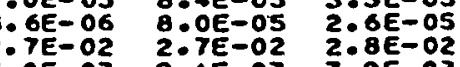

I.SE-03

$9: 4 E-03$
$9.2 E-03$

\begin{tabular}{l}
$.9 E-03$ \\
\hdashline$O E-03$
\end{tabular}

$7: 0 E=06$
$: 0 E-03$

$1 \cdot 7 E-03$

.

1.5E-03

$3.6 E-05$
$1.0 E-05$

$3.7 E-04$

:OE-02

$3.1 E-03$
$2.8 E-04$

2.2E-04

1.OE-OJ

8. $A E-03$

$: 5 E=03$
$: .6 E=03$

$2.1 E-05$

I.SE-OA

D.TE-05

$2.8 E-05$
$9.6 E-07$

$3.11-0.2$

ajE-03

$: 6 E-03$
$11 E-03$

3.7E-OA

$2.1 E-04$
$2.0 E-04$

$. J E-O 5$
$.4 E-O 5$

$8.0 E-04$

$6.2 E-04$

$4.6 E-08$

$3: 1 E=08$
$2: 1 E-06$

P.TE-06

$1.1 E-04$

$4.4 E-06$

$2.7 E-06$

:AE $=04$

$7.4 E-04$

ase-04

S. 
inHALATION DOSES AT I YFAP

(REM/MICHOCURIE)

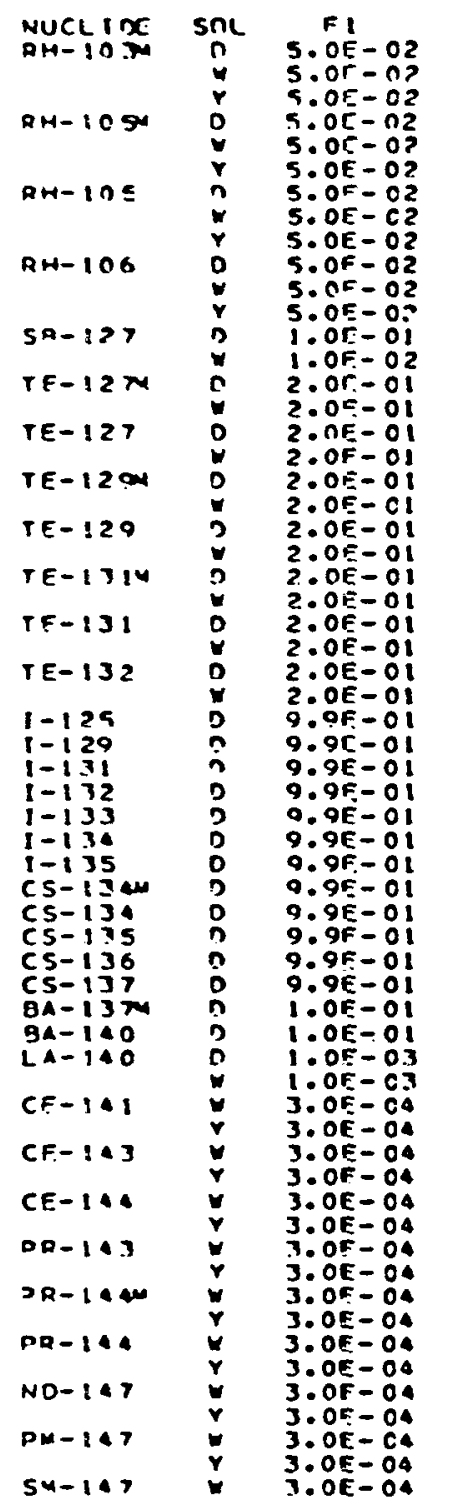

AMAO FFFECY L'NGGS 0.3 0.3 $0: 3$ $0: 3$ 0.3 0.1 0 0 $0: 3$ $0: 3$ $0: 3$ 0.3 0.3 $0: 3$ $0: 3$ $0: 3$ $8: 3$ 0.3 0.3 0.3 $0: 3$ $0: 3 \quad 3: 5 E-04 \quad 1: 7 E^{-0.03}$ $0: 3 \quad 1.5 E-04 \quad 0: 0 E-04$ $3.9 E-04$ $0.3 \quad 2.7 E-02 \quad 3.0 E-02$ $0.3 \quad 6.5 E-03 \quad 9.75-03$ $30 E-02 \quad 2.3 E-02$ $0.3 \quad 3.0 E-0\} \quad 9: 9 E-00$ $0.3 \quad 3.6 E-0.3 \quad 1.05-02$ $\begin{array}{lll}0.3 & 5.0 E-03 & 2.8 E-02 \\ 0.3 & 1.1 E-02 & i .1 E-02\end{array}$ $1.0 E-02$ 1.IE-OI $\begin{array}{lll}1.6 E-03 & 2.3 E-02 \\ 0.3 & 3.0 B E-0.3 & 2.55-02\end{array}$ $0.3 \quad 1.9 E-01 \quad 1.1 E 00$

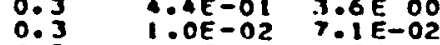

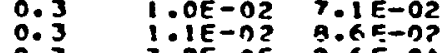
$\begin{array}{ll}0.3 E-05 & 2.7 E-04\end{array}$ 7.AE-OS 6.1 E- 04 $\begin{array}{lll}0.3 & 7.7 E-n 5 & 6.3 E-04 \\ 0.3 & B E=03 & 5.4 E-02\end{array}$ $0.3 \quad 8.05=0.36 .5 E$ 0.3 P.TEEOD $0.2 E-01$

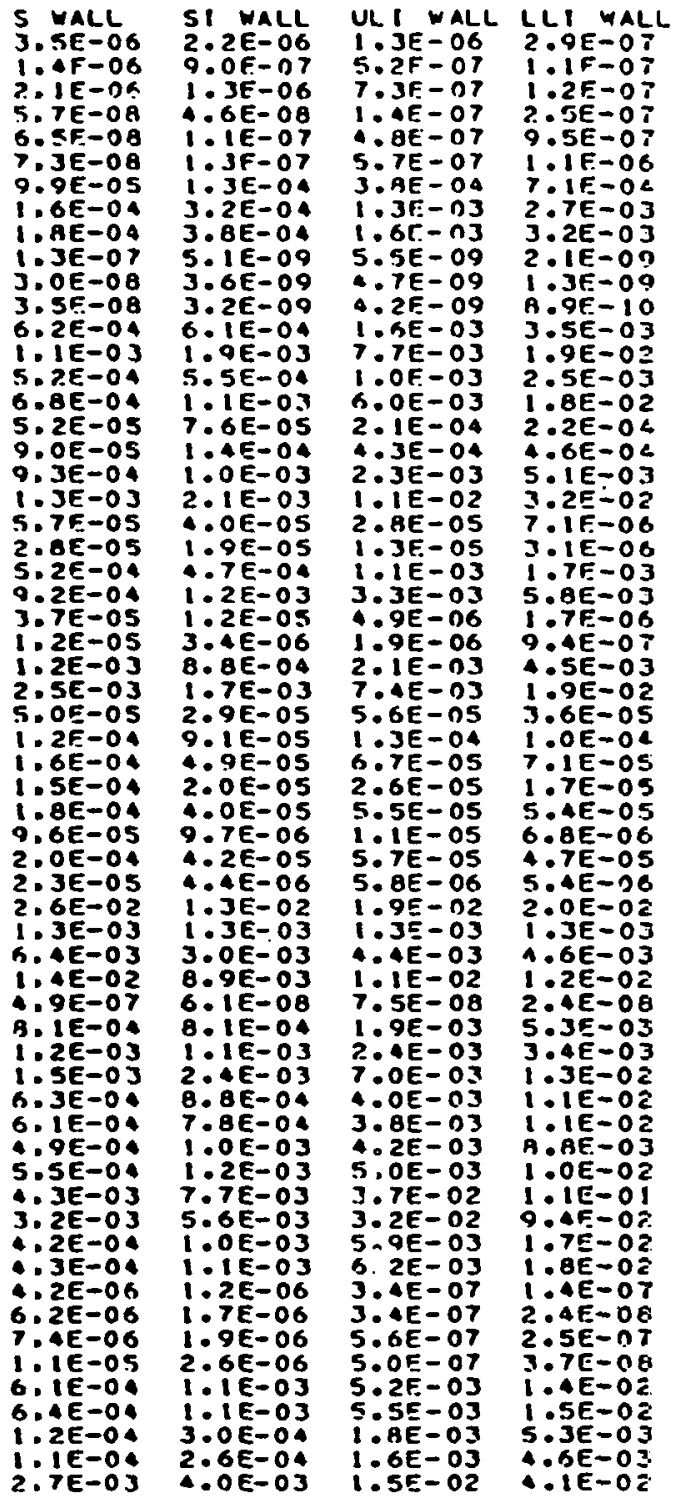

BL YALL 6. $\triangle F-n$ 2. IE-O)

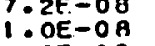
$1.2 E=0 B$
$1.2 E=00$ A $A \vec{E}-O B$ .IE-OA 1. IE-O 3. $3 E-05$ $2 . A E-O B$ $2: 5=-08$ B. 5 E -04 S. $\mathscr{T E - 0 4}$ $4.0 E-0$ $2 \cdot 3 E-05$ $1.7 E-03$ $4.95-06$ $2.2 E-06$ $5 . A E-04$ $4.5 E-06$ $1.6 E-0$ $1.0 E-03$ $6.2 E-05$
$1.9 E-04$ I:TE $: 04$ $T: 2 E=05$ $3: 3 E-0$ 1:3E-OA 9.6E 06 $3: 2 E-02$ $7.3 E-03$ $3.8 E-07$ $1: 2 E-03$ R. $6 E-04$ S. SE-O i. $3 E-0$. $1 . D E=04$ $3.5 E-0 J$
$1.3 E-0.03$ $5.9 E=06$
$2: 2 E=07$ 3. DE-O? $5.9 E-08$
$5.4 E-07$ I. $1 \mathrm{E}-07$ $3: 9 E-00$ $2.4 E-06$
$3.9 E-07$ 1. AE -03

2. $\mathrm{LE}-02$

6. AE -03

$2.6 E-0 \mathrm{~B}$ 9.2E 2 - 24 $\because 1 E-04$ 5. $1 E-05$ 6. OE- OS

2. $9 E-05$ 2. $7 E-04$ 5. $2 E=06$ 2. $4 E-07$ $1.1 E-08$
$\because 5 E-07$ 2.:1E-08 $6: 5 E-05$ 2. $2 E-07$
KIONEYS LIVFR 2.0E-nY 2.2E-n? $3.1 E-09$ ? $\triangle A E-00$ $1.3 E-O A$ ? $3 E-O$ $9.0 E-00 \quad 1.9 E-0$ 1.1E-0A 1:1E-04 3.:0E-05 $\quad$ A. $A E-0 S$ $2: 0 E-05$ J:AE-OS I: $2 E-00$ ?: $0 E-00$ I.IE-OA 3.5E-OB $7.6 E-04 \quad 2.9 E-03$ $3.6 E-04$ A.AE $3.4 E-04$ A.AE-O $\begin{array}{ll}2.3 E-05 & 2.3 E-05 \\ 5.2 E-06 & 5: 5 E-06\end{array}$ $1.6 E-03 \quad 1.6 E-03$ 5. $3 E-04 \quad 7.0 E-04$ $\because 5 E-06 \quad \because-9 E-06$ $1.7 E-06$
$7 E=-0.3 E=06$
$3: 3 E=04$ $3.6 E-04$ $3.2 E-06$

$1: A E-03$ $1: 1 E-03$
$5: 6 E-05$ 5.6E-0 $1: 0 E-04$ $\because .6 E-05$ $9.0 E-05$

2:0E-05

$8.9 E-0$

$3.2 E-02$
$2.6 E-03$
$2: 0 E-02$

I:OE-0J

6.SE 1.03

$3.3 E-04$

1:7E-:

$5.9 E-05$

6.ᄀE-O

$3.0 E-03$
$1.1 E-04$

$3.1 E-08$

$2.6 E-07$
$5.8 E-08$

1: $7 E=04$

$1.3 E-0.06$
$2.4 E-06$

i: $8 E-03$
QREAST, 2.?6:07 :DE-OB $1.6 E-08$ 1.JE-OA $3.2 E-05$ $2: 2 E-05$ i. $S E-08$ :IE-OA OOOE-O: IE-OA $5.2 E-06$ 6.6E-03 $4.6 E-06$ $9 E-06$
$: B E=0.8$ $1 E-04$
$3 E-0.06$ $3.8 E=06$
$3.0 E=06$ \begin{tabular}{ll}
$1 \cdot A E-03$ \\
\hdashline$E-03$
\end{tabular} $2 . B E-0$ $4.4 E-04$ 5. $1 E-05$

2. $2 E-0 S$

$1: 1 E-04$ $8.9 E-06$
$2.9 E-02$ $2.9 E-02$
$2.0 E-03$ $6.7 E-03$ $1.9 E-02$ 9.9E-0. B.OE -04 ऊ. $2 E-04$ 2.6E=-04 7.3E-05 $3.0 E-03$
$9: 1 E=04$ 5. $9 \varepsilon=06$ $2.2 E=07$
$2: 9 E-07$ $6.0 E-08$
$5.1 E-07$ Q.AE-OE $1.9 E-04$ $1: 9 E-04$
$3: 4 E-06$
$1.0 E=07$ 3. $8 E=03$ 
(REMPMICROCURIE)

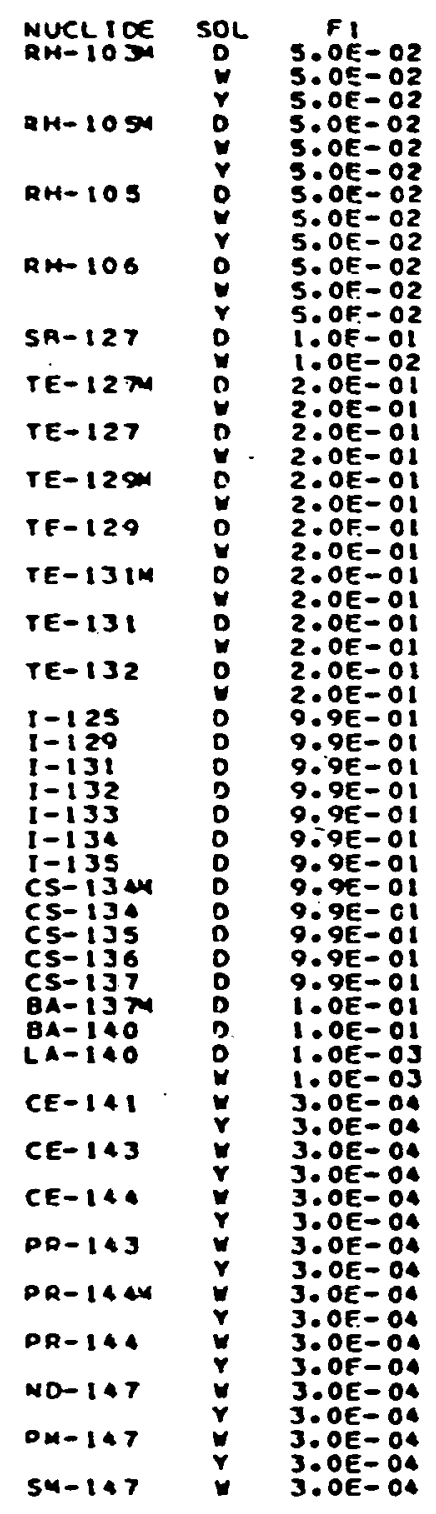

\begin{tabular}{|c|c|}
\hline 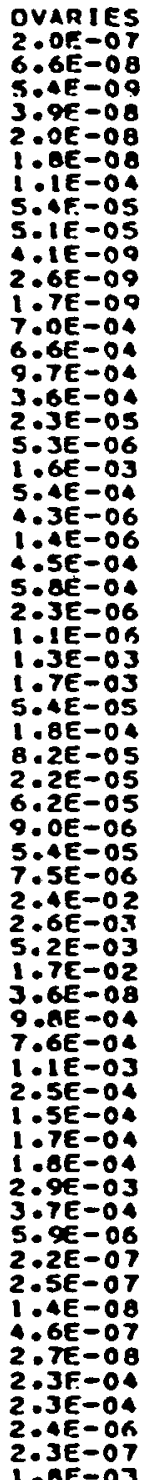 & 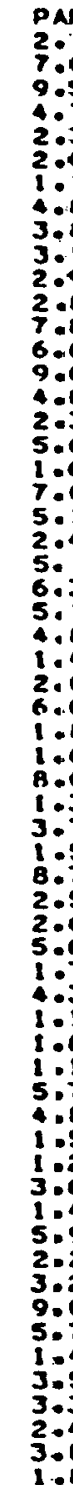 \\
\hline
\end{tabular}

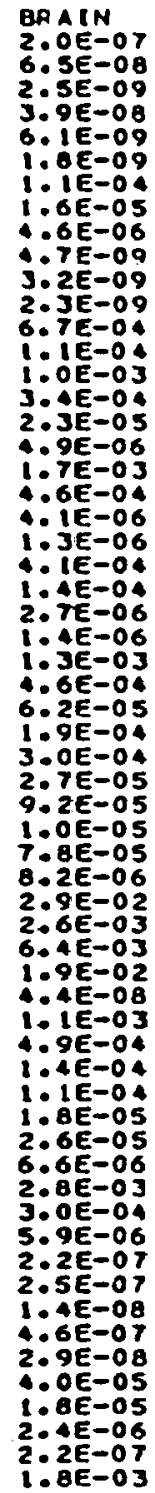

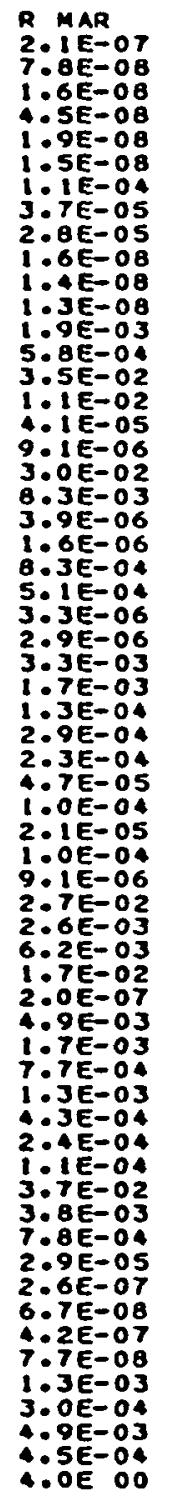

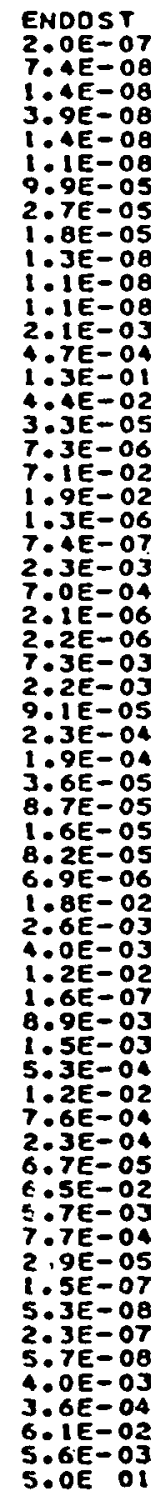

SKIN

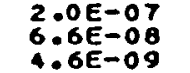
$3.6 E-09$
$1.8 E-0 B$ $9.4 \in E=09$ $1:$ i 1 - 04 $2: 2 E-05$
$1: 1 E-05$ $9.6 E-09$ $8.26-09$ 7:3E- 75 9:6E-04 3.AE-04 5:0E-0.5 1:6E:-03 $4,3 E-06$ $1.5 E=06$ $4.2 E-04$ 年: $2: 0 E-06$ $7.7 \in=04$ $\frac{1}{3}: 5 E-04$ $2: 1 E=04$ $9: 2 E-05$ \&.:5E-05 a: $5 E-06$ 2:9E-02 $6: 6 \mathrm{E}=03$ $1: 9 E=02$ $1.2 E-07$ 5.9E-O4 3.AE=04 1:OE=04 $3: 1 E=05$ $2,8 E-03$ 5.2E-D $5.9 E-06$
$2.2 E=07$ $2.9 E-08$ :9E=07 $5.3 E-08$
$8.6 E-05$ $8.6 E-05$
$7.7 E-05$ $2.4 E-06$
$2.5 E-07$ 2.SEE-03
SPLEEN TESTES $\begin{array}{ll}8 E-08 & 6.5 E-08 \\ : B E-08 & 2.5 E-09\end{array}$ .1E-0B 6.6E-09 REE 4 : $1 E-05$ I: $8 E-05$ $2:$ SE-08 2 . $7 E-09$

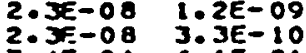
5. $4 E-04 \quad 6: 1 E=0$ 9.SE-O4 9. SE-OA $4.2 E=04$ J. $2 E=04$ 3. $4 E-06 \quad 4.9 E-06$ 6.TE-04 $4.3 E=04$ 2: $2 E=06 \quad$ i:1E-06 5. IE-04 3:AE-04 $\begin{array}{ll}3.6 E-04 & 1.2 E-04 \\ 4.5 E-06 & 2.1 E-06\end{array}$ : $: 4 E-03$ 1:IE-03

$1 . T E-03$
$1.2 E-04$
$3 E-05$
$1.5 E-05$ TE -04 $7: \begin{array}{ll}10-02 \\ 1: 0 E-05\end{array}$

$3: 3 E-05$

$1.3 E=04$
$9.2 E=06$

3.OE -02

1.9E-02

年

9

B. $2 E=03$

$1: 2 E=03$

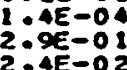

$2.4 E-02$
$5: 9 E-06$
$3,2 E-07$

$3.2 E-07$
$3.2 E-07$

8. TE=08

$2.5 E-07$
$2.6 E-0.4$

$2.6 E-0.4$
$2.8 E-04$
$3.0 E-04$

$2.4 E-06$
$3: 6 E=07$
$1.8 E-03$

$1.7 E-04$ $1.9 E-055$
$5.9 E-05$

$7.4 E-06$

T. $5 E=05$ $2.5 E-02$
$2.6 E-03$ 5. $6 E=03$ $1.5 E-0 B$

$8.9 E-04$ $1.5 E-04$

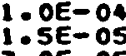
3. $0 E=05$

$2.7 E-03$
$2.3 E=04$

2.3E- 34

$2.2 E-07$
$2.4 E-07$

$9.4 E=09$
$4.5 E-07$

$1.8 E-08$
$2.9 E-05$

. BE-05

$2.3 E-06$
$2.2 E-07$

THYMUS $7.6 E-08$ 2.0E-0?

-5E-08 2.7E-09 5. 阮 $5.5 E-05$
$: 5 E-05$ $42 E-08$ $41 E-08$
$4.0 E-08$ $7.5 E=04$ $9.3 E-04$ $4.6 E-04$
$2.3 E-05$ $2.3 E-05$
$5.7 E-06$ $1.6 E-03$

$5.2 E-06$

2: $3 E-06$ $8.9 E-04$ 7. $3 E-06$ $1.5 E-03$
$2.6 E-03$ 2. $4 E-04$ Y. $\mathrm{AE}-04$ 1: $1 E-04$ $5.0 E-05$ $2.3 E-04$
$9.1 E-06$ $2.6 E-02$
$2.6 E-03$ 2. $6 E=03$ $1.7 E-02$
5. $9 E-07$ $9: \begin{array}{ll}0=04 \\ 9 E-0 \circ\end{array}$ $1.4 E-03$

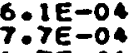
$1.7 E-04$ $1.5 E-04$
$3.4 E-03$ $2.3 E-03$
$5.9 E-06$ 2.2E-07 1. $4 E-07$

$6.5 E-07$
$2.2 E-07$

$4.2 E-04$
$4.6 E-04$ $4.6 E=04$
$2.4 E=06$
$4.9 E-07$

$2: 9 E-07$
$1.0 E-03$
$1.8 E-0.07$

UTERUS 2.0E-0.?
$6.5 E-0.0$ 3.5E=09 i. 1 TE-OB

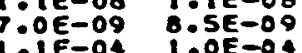
$\begin{aligned} & 2.5 E-05 \\ & : 4 E-05\end{aligned} 2: 1 E-05$ $\because 5 E-08 \quad 3.5 E-09$ $1 . J E-08$ 1.2E-O9 $3.2 E=04$ 3.2E=: $3.4 E-04$ 3.2E-OA $5.1 E-06$ S:1E 06 $1.6 E-03$ .AE-06 $\because: 2 E=06$ $3: 3 E-02 \quad 3: A E-04$ $5: A E-02 \quad 3: 1 E=04$ $11 E-03 \quad 1.0 E-06$ $3.3 E-03 \quad 8.4 E-04$ 6. $A E-01 \quad 5.2 E-05$ 9.7E-OI B:OE-OS 2.2E-01 2.1E-OS .7E-04 8.7E-06 S: $6 E=02$ 3. $8.2 E=03$
$2.0 E-02$ 2. $1 E-07$

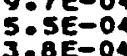
3. .6E-O 3. $9 E-05$ $2.9 E-03$ 5. $9 E-06$ $2: 2 E-07$
$2: T E-07$ 5.6E-08 a. $6 E-07$ 作 Q 
INAALATION DOSEES AT I VEAR

(HEMTUICROCURIE)

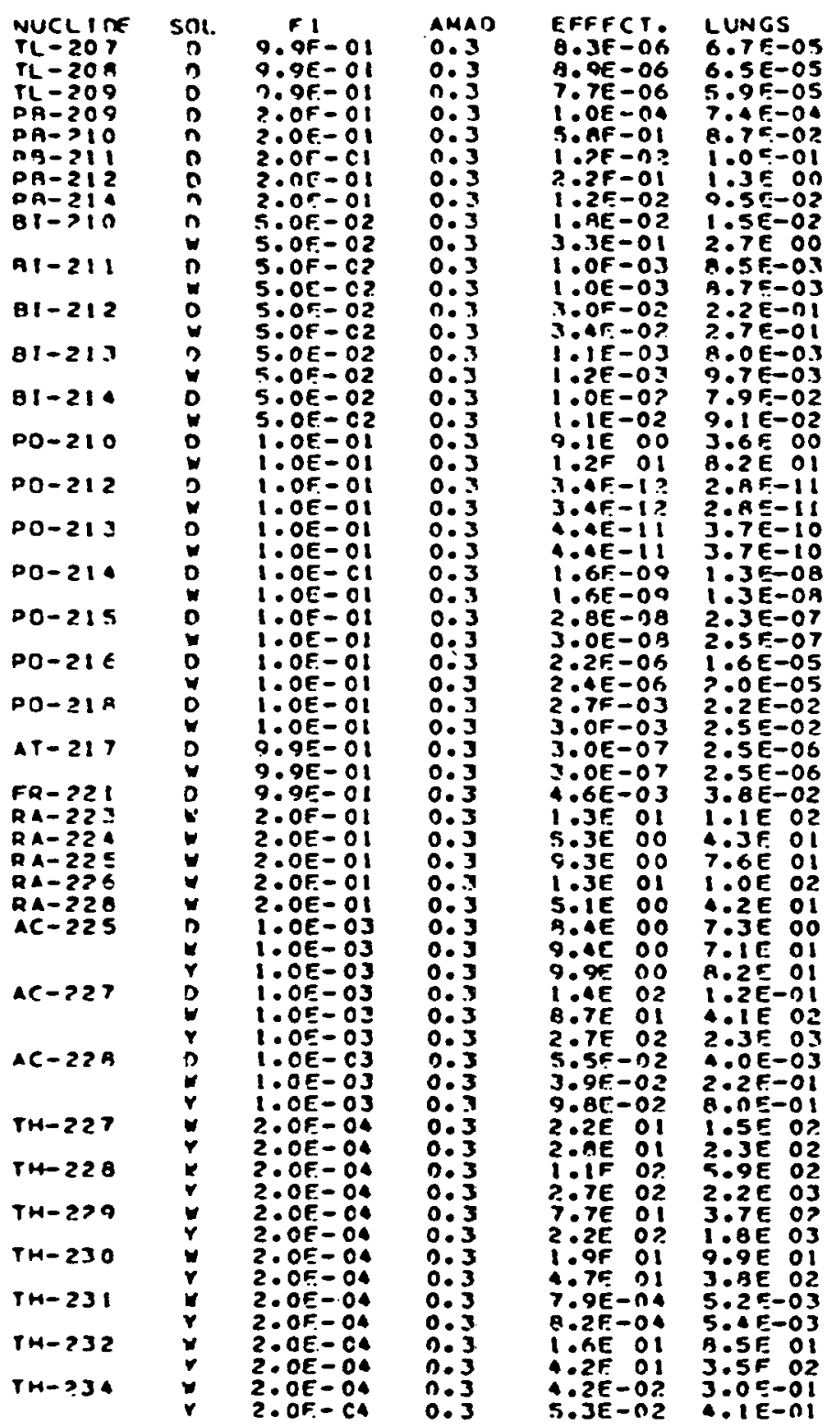

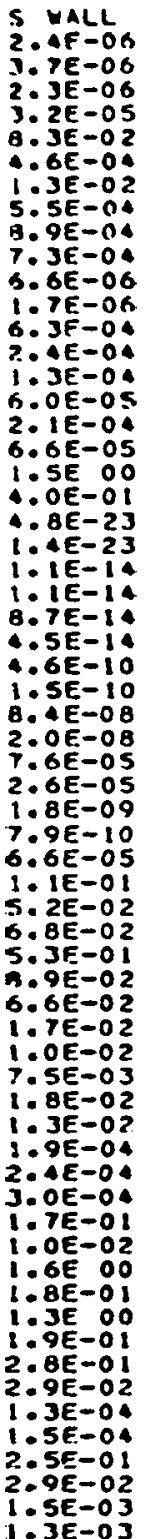
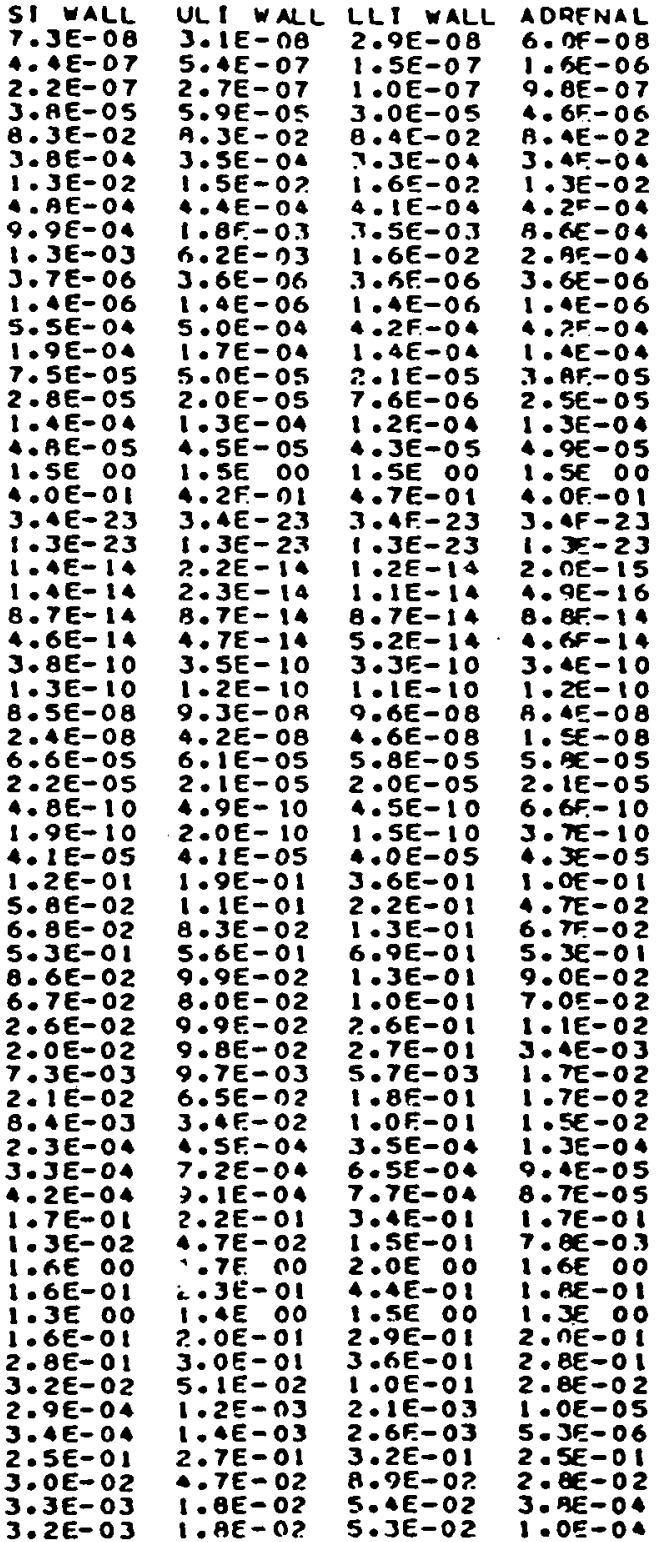

OL WALL

S.
2. $0 E-07$

$1.5 E-07$
$4.6 E-06$

B. $4 E-02$
$3.4 E-02$

$3.4 F-04$
$1.3 E-0: 5$

$1.1 E-04$
$B$.

2. $\theta E-04$

$3.6 E-D K$
$1 . A E-D Q 6$

$4.1 F=04$

1. $A E-05$

6. $5 E-06$

$1.3 E-04$
$4.3 E-05$

i.:SE-05

$4.0 E-01$
$3.0 E-23$

2. $0 E-15$

A. $9 E-16$

$4.6 E-14$

$1: 2 E-10$

8.JE-OB

5. $B E-05$

$2: 0 E-05$
$4: 9 E-10$

$1 . P E-10$

$1.0 E-01$

$4.6 E-02$
$6.5 E-02$

$6.5 E-02$
$5.3 E-01$

$8.3 E-02$
$6.4 E-02$

B. IE-03

3. OE 03

1.: $1 E=02$

2. $3 E-03$

2. $7 E-05$

1:9E=-05

6.7E-03

:SE-OI

I. उE DO

2. $\mathrm{AE}-\mathrm{O}$

7.:0E-06

$2.4 E-06$
$2.5 E-01$

2. $7 E-02$

KIONEYS

$3.2 E-06$

LIVEA

S. BE-08
$2.1 E-06$

1:1E-06

$1: 8 E$ OO

$7: 0 E-02$

$3.9 E 00$

T:SE-O1

.5E-01 8.6E-0

$8.0 E-02 \quad 2$. TE-04

$1.6 \mathrm{E}-05$ B:IE-0B

2. $0 E-02$ B. $5 E-05$

$9 E-03$

2: TE-OS

9.3E-03 3:0E-OS

3.3E-03 $1:$ BE-05

(1)

$7:$ TE 00
$2: 0 E$ DO

$3.8 E-27$
$1.5 E-27$

5. $4 E-26$

1.6E-14 $2.7 E-15$

$2: 7 E-12$

$6: 7 E-16$

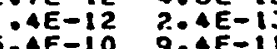

9E-10 3.JE- 11

$2.6 E-07$ : $5 E-08$

(1)

2:DE-0S

$1.9 E-10 \quad 2: 7 E-10$

2: $A E-100$

3.2E-02

$3: 0 E-06$

6.4E-02

B. $1 E-02$

$6: 4 E-02$

8.6E-02 8. $9 \mathrm{E}-02$

$3.6 E:$

$1.9 E-03$

A:IE-OO

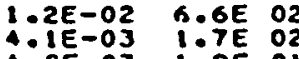

$4.88-03$

$1.9 E$ O

5. OE-05 $6.1 E-02$

S.0E-05 S.TE-0.3

$6.5 E-03$

$5: 3 E-02$

$1.6 E-01$

I.JE

I.JE DO $1:$ IE OI

$2.8 E-O 1$

$1.2 E 00$

3. $4 E-06$

2.3E-OI

$\begin{array}{ll}2: 1 E-06 & 7.0 E-06 \\ 2.4 E-01 & 2.0 E-00\end{array}$

$2.6 E-02$

$2.0 E$
$2.2 E-01$

a.TE-OS 1.BE-OA

BAEAST

$1.3 E-06$
$6.7 E-0 ?$
$A: 3 E-08$ $A, \triangle E-02$
$3, j E-0,02$ $3: \because E=04$

IE-OA

$.6 E-04$
$3.0 E-04$

$3.66-06$

I. $2 E=0$

$2.6 E-05$

$1.7 E-O S$

1.2e

$1.5 \mathrm{SE}$.

$3.4 \mathrm{~B}-23$

$1 \cdot 3 E-23$

$2.0 E-15$
$: .9 E-16$

$8.8 E-14$

$3.3 E-10$

$1.2 E-10$
$B .3 E-08$

$1.5 E-08$

$5.9 E-10$

T.OB

$1.0 \mathrm{OE}-01$

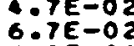

के

$6.5 E-02$

2.5E

B. $3 E-02$

$8.8 E-03$

$6.1 E-05$

S.7E-OS

T.TE
$1.3 E=01$

$1.6 E$ OO

I.TE-O1

1:BE-O:

2. $8 E-01$

$1.1 E-05$
$6.7 E-06$
$2.5 E-0.1$

$2.5 E-01$
$2.0 E-02$

S. 


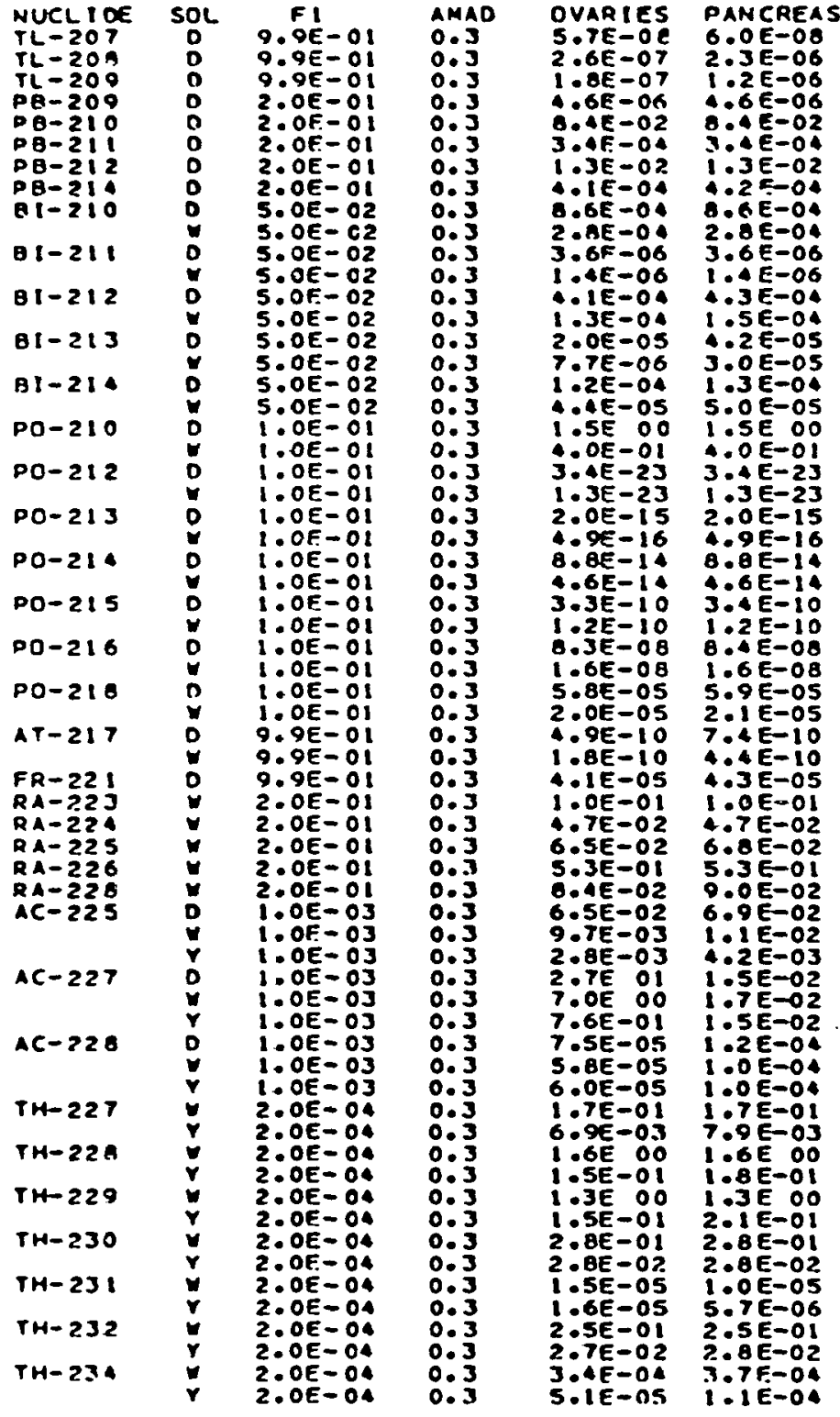

(REMAMICROCURIE)

BRAIN

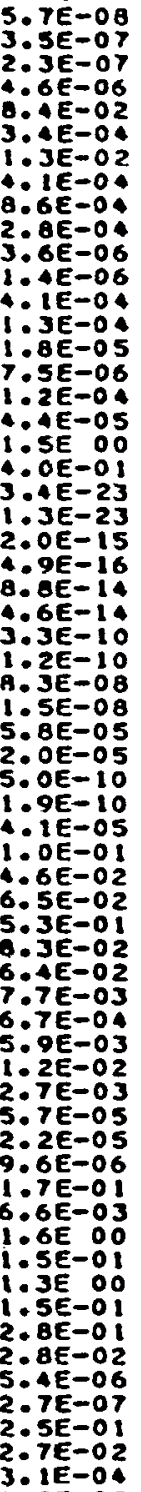

R. MAR $1.2 E-06$
$7.0 E-O T$
3

$0.4 E-08$
$6.06-07$ $9.6 E-07$
$1.2 E-06$ $3.6 E-05$ 1.2E OO $1.1 E-01$ $1.3 E-03$ $1.1 E-04$
$2.6 E-06$ $9.9 E-07$ $4.6 E-04$
$1.5 E-04$ $2.8 E-05$ 1:PE-OS :. $2 E-05$ 2. $B E-01$ $2.3 E-23$ $1: 7 \in-15$ 1:JE-13 $6: 9 E-14$ 8: $3 E-11$

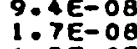
$4.2 E-05$ $1.5 E-05$ $4.8 E-10$
$2.6 E-10$ $2.9 E-05$ 3.6E-0 3.: $9: 8 E-02$ I.IE :I 5.2E-OO $2.0 E$ O2 $5.6 E$ OO $7: \theta E-02$
$1: \theta E-02$ T:PE-O 7:1E 110 $7.7 E$
$6.9 E$ 01 6.AE OI G.BE OO 6.3E 000 $1,1 E-05$ $1.3 E$. DO $\begin{array}{ll}1.10-05 & 5.2 E-02 \\ 1.8 E-04 & \end{array}$ 411
$4: 6=07$
$36=06$ a: $4 E-02$ $1.2 E 00 \quad 1.3 E-02$ $3.2 E-03$ A. $1 E-04$ 2.7E-03 2.8E-O4 $1.4 E-06 \quad 3.6 E-06$ $2.2 E-03$ A.1E-04 $6.9 E-04 \quad 1.3 E-04$ $6.5 E-05$ $2.8 E-05$
$3: 3 E-04$ $1.2 E-04$ $2.1 E-01$
$3.2 E-33$
$1.0 E-01$

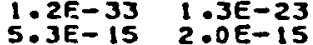
$\begin{array}{ll}5.3 E-15 & 2.0 E-15 \\ 1.3 E-15 & 4.9 E-16\end{array}$ $9.0 E-13$ B.BE-14 $\begin{array}{ll}: 7 E-13 \\ 1.0 E-10 & 4.6 E-14 \\ 6 & .3 E=-10\end{array}$ $6.4 E-11$
$4.7 E-07$
$6.3 E-10$ B. $8 E-0 B \quad 1: 5 E-08$ $1.4 E-05 \quad 2.0 E-05$ 5. $1 E-10 \quad 5.4 E-10$ $2.4 E-10$ 7. $3 E-06$ -3E DO 1:OE-OI $4.3 E$ DO $4.6 E-02$ $1.3 E$ OO $5.3 E-01$ 4.5E-01 8.5E-02 I.5E of B.JE-O $6.1 E-01 \quad 1.4 E-03$ $6.4 E$ O2 $1: 2 E-02$ $6.9 E$ O1 $5.0 E-03$ $\begin{array}{ll}9 \cdot 9 E-01 & 6.4 E-05 \\ 2.2 E-01 & 3.7 E-05\end{array}$ $2,1 E-02$ 3. $1 E$ OO B.5E OI 1.6E-OI $7.9 E$ O2 $1.3 E$ O. OO $\begin{array}{lll}8.3 E & 1 & 1.6 E-01 \\ 1.6 E & 02 & 2.0 E-01 \\ 1.0 E & 01 & 2.0 E-02\end{array}$ $\begin{array}{ll}1.6 E & \text { of } \\ 6.6 E-04 & 2.6 E-02 \\ 6.2 E-06 & 0.05\end{array}$ $2.9 E-05 \quad 1.3 E-06$

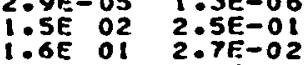
$2: 1 E-02$
$2.2 E-04$
$8.7 E-04$
$3.5 E-05$
SPLEEN TESTES 5.7E-0B I:AE-OT

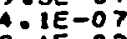
5. $9 E-06 \quad 3.4 E-04$ $1.8 E-05 \quad 91 E-04$ $\begin{array}{ll}2.7 E-04 & 8.6 E-04 \\ 2.0 E-04 & 2.8 E-04\end{array}$ $1.7 E-07 \quad 3.6 E-06$ 2. उE-04 A. $1 E-04$ B.SE-05 $1: 3 E-04$ $\begin{array}{ll}2 \cdot 8 E-05 & 1.6 E-05 \\ 2: 2 E-05 & 5 . T E-06\end{array}$

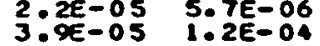
1.0 $\frac{\theta E-05}{7 E} 01.3 E-05$ $2.1 E$ OI $\quad 40 E-01$ $2: 1 E-26 \quad 1.3 E-23$ 2. TE-14 2.OE-15 $46 E-12$ 8.8E-14 $2: 4 E-12 \quad 4: 6 E-14$ $3.2 E-10$
$2.2 E-1.2 E-10$
$4.3 E-06$ $4: 4 E=07$ 1. $5 E-08$ 6.TE-05 2.0E-05 $2 . T E-10$ A: $9 E-10$ $2.3 E-10 \quad 1.7 E-10$ $8.1 E-02$ 1.OE-01 $6.4 E-02$ 6.5E-02 5. JE-OI $5.3 E-01$ $\begin{array}{ll}8.8 E-02 & 8.2 E-02 \\ 2: 3 E-03 & 6.3 E-02\end{array}$ $2.0 E-03 \quad 7.5 E-03$ $\begin{array}{ll}3.0 E-03 & 5.6 E-04 \\ 4 & 3 E-03\end{array}$ 3.: $2 E-03$ $1.1 E-02$ 2: ?E 01 $7.6 E-01$
$4.0 E-05$ $7.8 E-05 \quad i: 7 E-05$ B.EE-O5
$1.0 E-01$ $\begin{array}{ll}1.6 E-01 & 1.7 E-01 \\ 7.2 E-03 & 6.0 E-03\end{array}$ T:ZE-03 1. $6 \mathrm{E} 00$ 1:JE-D 1:-5EE-OD $\begin{array}{ll}1: 9 E-01 & 1: A E-01 \\ 2.0 E-01 & 2.0 E-01\end{array}$ $\begin{array}{ll}2.8 E-02 & 2.8 E-02 \\ 6.7 E-06 & 2.0 E-06\end{array}$ S. TE-06 A.KE-07

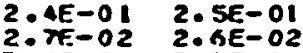
$3: 6 E-0.43$
$0 . T E-05$
THYMUS $6.1 E-08$
$3.5 E-06$
$3.6 E-06$ $1.9 E-06$
$1.6 E-06$ B. $A E-02$ $1.3 E-02$ $.2 E-04$
O. $2.8 E-04$ $1: 7 E=06$
$1.4 E=0.06$ $43 E-04$ 1. $6 E=04$ i. $2 E-05$ $5 . A E-05$
$1.5 E-00$ 1. OE-OI 1.: $3 E-23$ 3.4E-23 $2.0 E-15 \quad 2: 0 E-15$ $8.9 E-16$ $4.6 E-14$ 3:AE-10 i. 1 EE-OB $5: 9 E-05$ : $2 E-10$ $5.9 E-10$

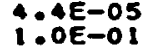
$1: 0 E-01$
$\because 7 E-02$
$S E S E-02$ 5: 0 : $3-02$ $9.6 E-02$ $1.2 E-02$ $1.3 E-03$
$2.3 E-02$ $1: 5 E-02$ $2.2 E-02$ $1.4 E-04$
$1.5 E-04$ 1:TE-OI $1.7 E-01$
B. $6 E-03$ $1.6 E .0$ 1.3E 00 I:JE $\begin{array}{ll}2.3 E-01 & 1.7 E-01 \\ 2 . B E-01 & 2.8 E-01\end{array}$ $\begin{array}{ll}2.8 E-02 & 2.8 E-02 \\ 1.3 E-05 & 6: 3 E-00\end{array}$ $7.9 E-06$ $2.5 E-01$
$3.0 E-02$ $4.1 E=04$
$1.7 E-04$

$2.5 E-01$
$2.7 E-02$ S. IE- 05
I. $3 E-06$

UTERUS SHE-DQ 5.7E-0. :2E-07 $2: 1 E-0$ T B. $4 E-02 \quad 8.4 E-02$ - उE-02 $1.3 E-02$ .6E-04 8.6E-04 $3.6 E-06 \quad 3306-06$ 1E-04 $1.1 E-04$ $\begin{aligned} & 1: 4 E-04 \quad 103 E-04 \\ & 2: 3 E-05\end{aligned} 2: 0 E-05$ OAE-05 B.1E-06 1.2E $4: 0 E-101$
$3: 4 E-23$ $1 \cdot 3 E-23$ $2: 0 E-15$
$: 0 E-16$ $8.8 E-14$ $3.3 E=10$ $1.2 E-10$
$8: 3 E-0 B$ $1.5 E-08$ $2.0 E-05$ $4.9 E-10$ $1 . B E-10$
$4.1 E-05$ $1.0 E-01$ $1.6 E-02$
$6.5 E-02$ S.SE $=01$ $8.3 E=02$
$6.4 E-0.02$ $6: A E-02$
$8: 5 E-03$ $1.6 E-03$ $3.7 E-03$ $2.5 E-03$ $40 E-05$ 3.7e$1.7 E-01$
$6.7 E-03$ 1.6E I. $3 E$ OO $1.5 E-0$
$2.8 E-01$ $2.8 E=02$
$3.2 E=06$ $3.9 E-06$ $2.5 E-01$
$2.7 E-02$ 3.2E
$2.9 E-0.03$ 
InIALATION DOSES AT I VEAR (FEM/MICROCURIE)

\begin{tabular}{|c|c|c|c|c|c|c|c|c|c|c|c|c|c|c|}
\hline $\begin{array}{l}P A-233 \\
P A-2344 \\
P A-2.34 \\
U-2.32 \\
U-233 \\
U-234 \\
U-235 \\
U-236 \\
U-237 \\
U-239 \\
U-240 \\
N P-237 \\
N D=239 \\
N P=239 \\
N P-2404 \\
D U-236 \\
P U-23 A \\
P U-239 \\
P U-240 \\
P U-241 \\
P U-242 \\
P U-243 \\
P U-244\end{array}$ & 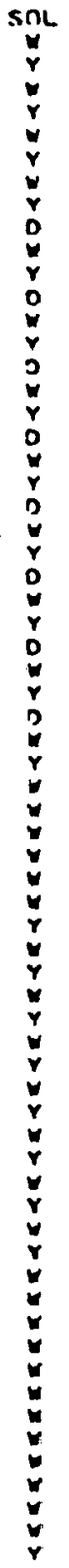 & 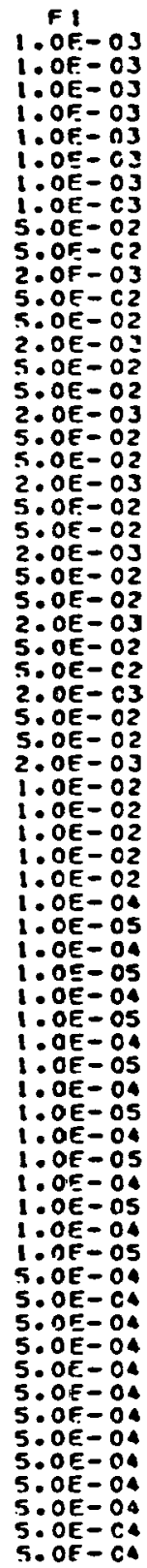 & $\begin{array}{l}A=10 \\
0: 3 \\
0: 3 \\
0: 3 \\
0: 3 \\
0: 3 \\
0: 3 \\
0: 3 \\
0: 3 \\
0: 3 \\
0: 3 \\
0: 3 \\
0: 3 \\
0: 3 \\
0: 3 \\
0: 3 \\
0: 3 \\
0: 3 \\
0: 3 \\
0: 3 \\
0: 3 \\
0: 3 \\
0: 3 \\
0: 3 \\
0: 3 \\
0: 3 \\
0: 3 \\
0: 3 \\
0: 3 \\
0: 3 \\
0: 3 \\
0: 3 \\
0: 3 \\
0: 3 \\
0: 3 \\
0: 3 \\
0: 3 \\
0: 3 \\
0: 3 \\
0: 3 \\
0: 3 \\
0: 3 \\
0: 3 \\
0: 3 \\
0: 3 \\
0: 3 \\
0: 3 \\
0: 3 \\
0: 3 \\
0: 3 \\
0: 3 \\
0: 3 \\
0: 3 \\
0: 3 \\
0: 3 \\
0: 3 \\
0: 3 \\
0: 3 \\
0: 3 \\
0: 3\end{array}$ & 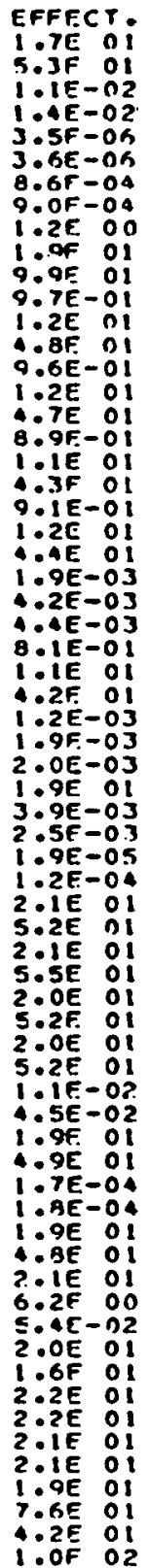 & 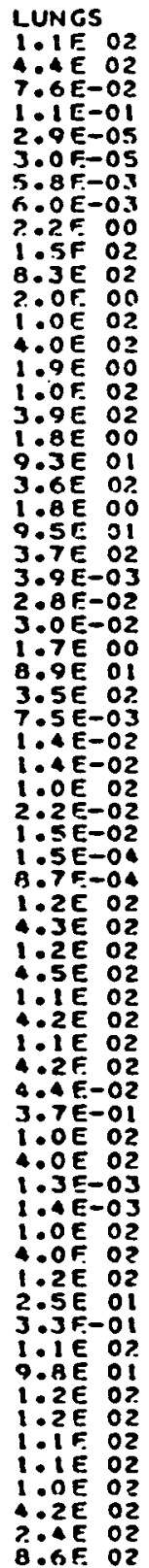 & 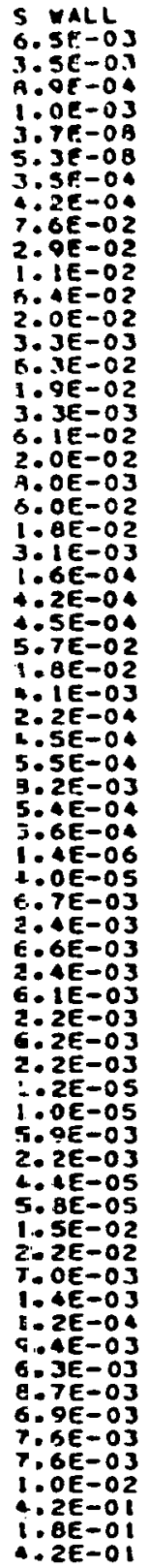 & 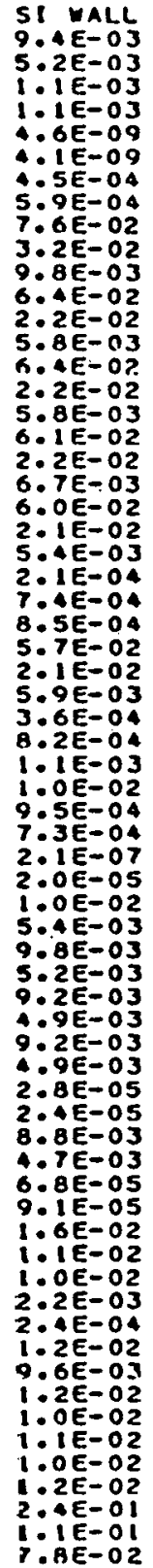 & 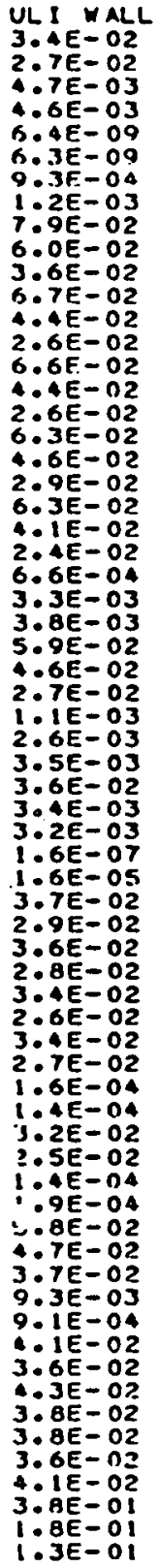 & 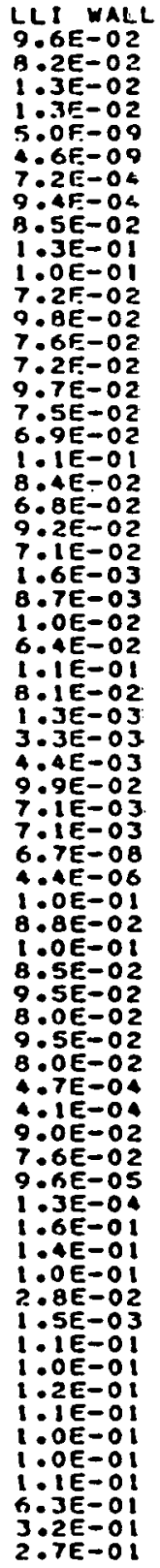 & 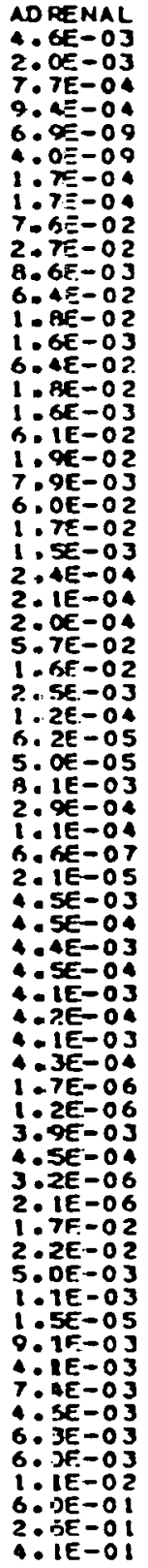 & 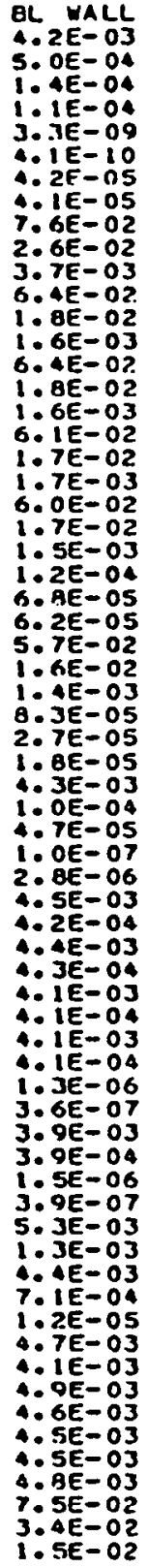 & 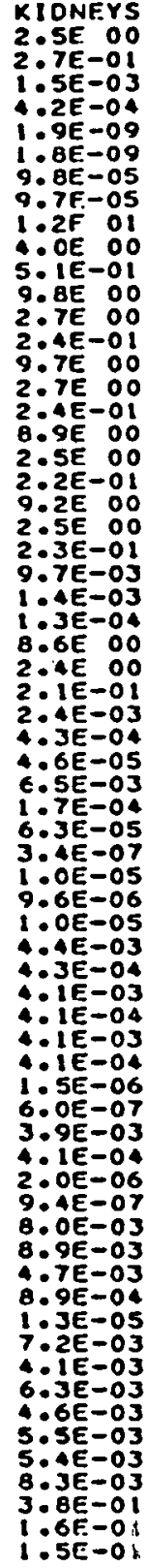 & 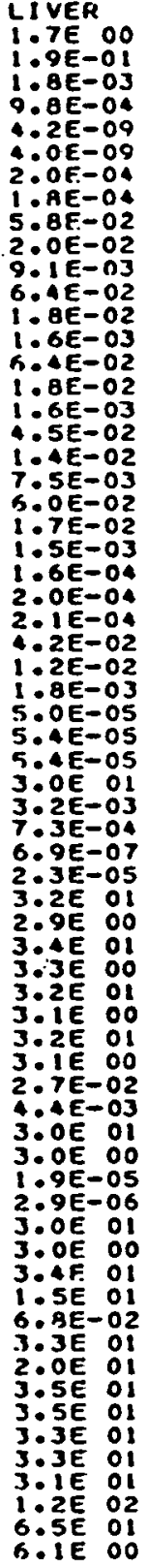 & 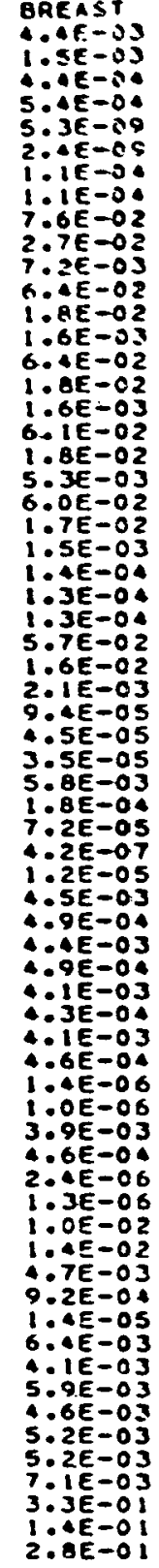 \\
\hline
\end{tabular}


INMALATION DOSES AT I YEAP

(REM/AICROCURIE)

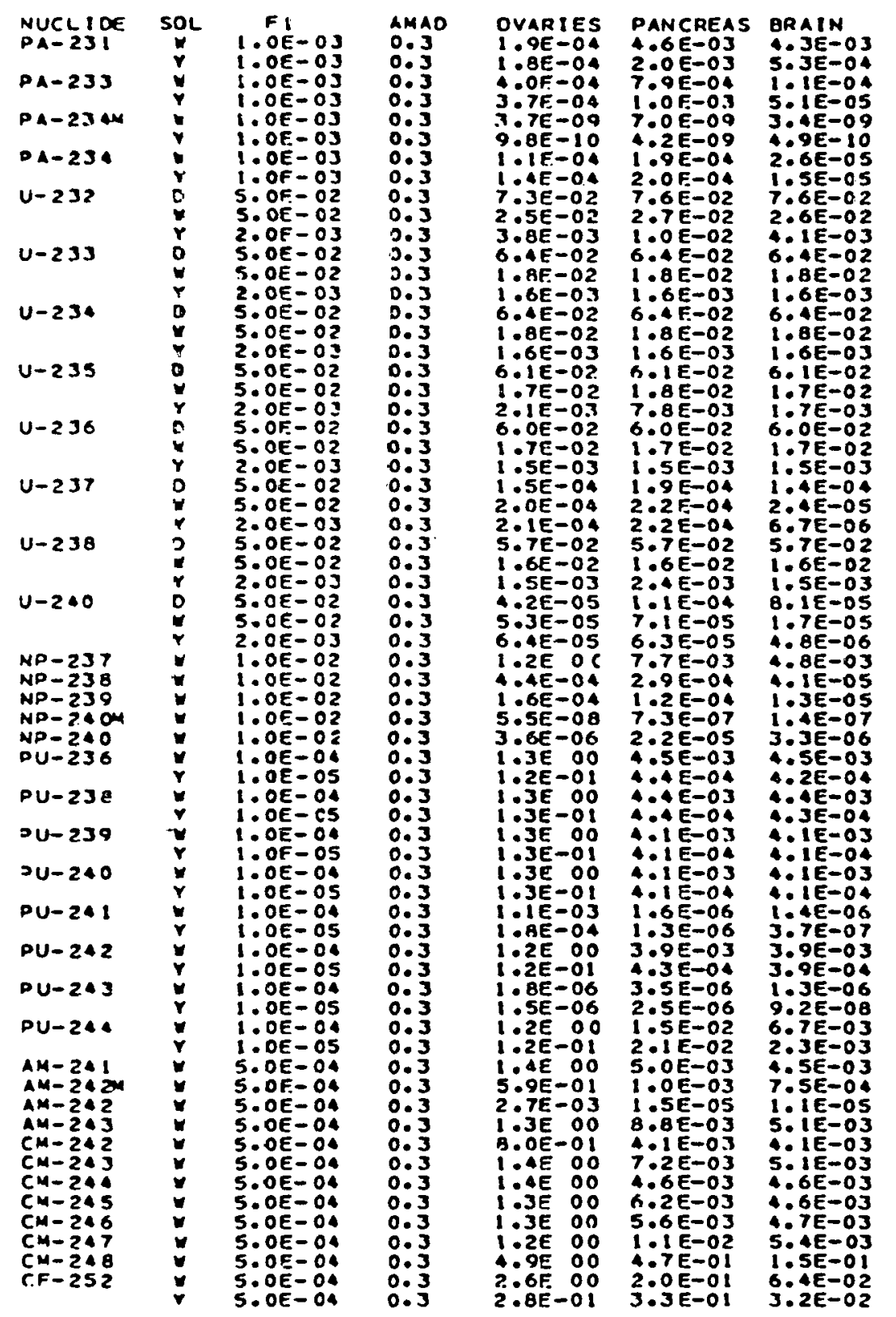

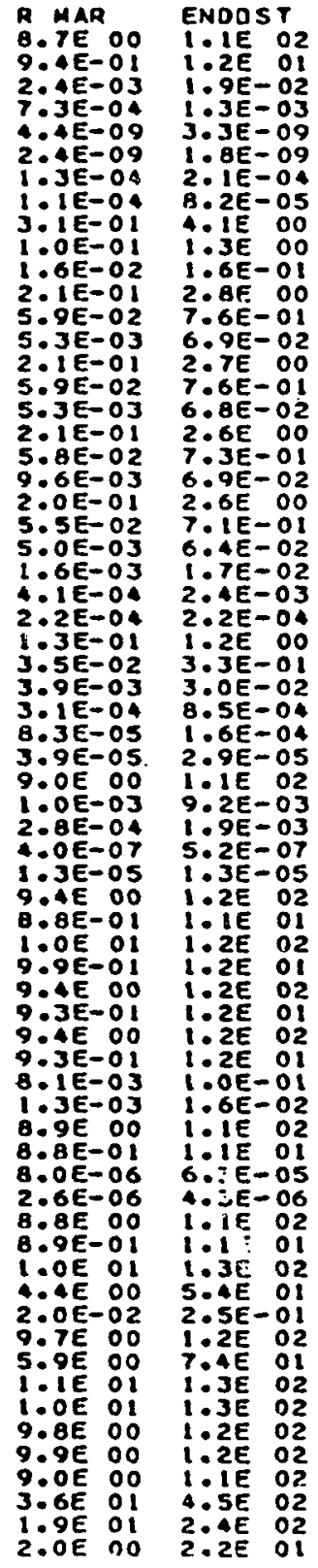

SKIN

$4.2 E-03$

SPLEEN TESTES

6. 2 2E-05

THYMUS

THYRO

$2.0 E-O 4$ O

$3.3 \mathrm{E}-05$

$1: 3 E-09$

B. $6 E=04$

3. $1 E-00$

$3.8 E-09$

2. $0 E-10$

$\begin{array}{lll}5.2 E-05 & 1: 7 E-04 & 1: 1 E-05 \\ 7: 6 E-02 & 5: 0 E-02 & 5: .8 E-02\end{array}$

2.6E -02

$5.8 E-02$

2: $0 \mathrm{OE}-02$

$5.3 E-03$

Q: $7 E-03$

政

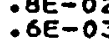

$1.8 E-02$

$1: B E-02$

1.AE-02 $\quad 6.4 E-02 \quad 6.4 E-02$

$1.8 E-023$

$1.8 E-02$

1: $8 \mathrm{BE}-02$

$1: 7 E-02$

1.: $B E=02$

6.: 1 EE-O2

$2.9 E-03$
$6.0 \mathrm{E}-02$

$7.4 E-093$
$0.0 E-025$

$1: 06 E-03$

$1.7 E-02$
$1.5 E-03$

1: TE $1: 02$

: $: 7 E-02$

$5.6 E-05$ 2:0E-OA $2.2 E-05$

$4.6 \mathrm{E}-05$

$1: 9 E-0$

1: $5 E-05$

$1.6 \mathrm{E}-02$

$1.7 E-02$
$1.6 E-02$
$1.3 E-03$

$5.7 E-02$
$1.6 E-02$

$\begin{array}{ll}1: 3 E=03 & 1.4 E-03 \\ 1: 1 E-04 & 2: A E-05 \\ 6.0 E-05 & 7.7 E-006\end{array}$

$2.8 E-05$

$6.0 E-0$
$5,0 E-0$

$1.0 E-0 J$
a

6.0E-03 $1.2 E-06$

$3 \cdot 1 E-05$

$2.6 E-04$

1. $2 E$ O 00

$1.0 E-04$
$6: 1 E-07$

$7.1 E-06$
$: .5 E-03$

2. $0 E-05 \quad 2.2 E-O B$

$A .2 E-04$
$4.4 E-03$
$4.4 E$

\begin{tabular}{ll}
$: 1 E-O 5$ & $1: 3 E-O O$ \\
\hline $.9 E-05$ & $1.2 E-01$
\end{tabular}

AE-04 $\because: 5 E-04$ 1:JE-O

$\because \because 1 E=03$

$\because 1 E-03$

$1 .: 3 E-0 !$
$1.3 E$
0.30

IE-O

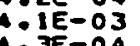

1:3E-00

$3.3 E=06$
$5.3 E=07$

1: $4 E-06$

$3: 3 E-01$
$1: B E=03$

$3.9 E-03$

$3: 2 E-06$
$: 9 E-03$

$1: B E-04$

$3.6 \mathrm{E}-06$
3.06

3. $1 E-D B$

$1.2 E-01$

$\begin{array}{lll}3.3 E-03 & 2: 1 E-06 & 0.8 E-08 \\ 0.0202 & 1.2 E-00\end{array}$

$6.6 E-03 \quad 1.8 E-02$

1.2E O O

$7.5 E-04$

4 . TE-O3

S.AE-DE

$1: 5 E-05$

2. $7 \in-03$

$5.1 E-03$
$4.2 E$

$4.1 E-03$
$6: 2 E-03$

: OE-OI

$5.2 E-03$
$4.6 E-03$

$4.6 \mathrm{E}-0 \mathrm{~J}$

T:AE $: 0$

$4.8 E-03$
$5.7 E-03$

$5.4 E-03$
$5.2 E-03$

1. JE OO

$\begin{array}{lll}1.9 E-01 & 3: 0 E-01\end{array}$

\begin{tabular}{rl}
$1.2 E$ & 00 \\
\hdashline $.9 E$ & 00
\end{tabular}

$7: 7 E-02$
$1.2 E-01$

$3: 2 E-0$ i

2. GE DO

1. $1 E=03$

$9.8 E-09$

$2.6 E-104$
$2.6 E-004$

$7.6 E-02$

$1.4 E-02$

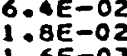

$1.6 E-03$
$6 E-02$

$0.4 E-02$
$1.0 E-02$
$6, O E-03$

(1E-O2

$1: 9 E-02$

.

: $: \mathrm{TE}-020$

$3.1 E-04$
$3.2 E-04$

5.7E-O2

$1.6 E-02$
$3: 1 E-03$
$3 .: A E-04$

$8: 4 E-05$

$7.5 E-05$
$6.9 E-03$

$1: 1 E-04$
$1: 5 E=04$

$1: 5 E-04$
$3: 0 E-06$

3. $3 E-05$

$\because 66=04$

:SE-O

$41 E=03$

$41 E-03$

1 SE-O6

$1.8 E-06$

$3.9 E-03$

$\because: 4 E-06$

$1.4 E-02$

$3.3 E-02$

O:PE-DJ

$8.3 E-03$
$1: 6 E-05$

$\therefore 1 E-03$

$6.0 E-03$

$6.1 E-0 J$

$5.5 E-0 J$
$9: 0 E-0 J$

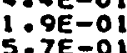

$1.0 E-03$

更

$2.2 E-09$

. $2 E-05$

$2.6 E-02$

$6.4 E-02$

$1.8 E-02$

$1.6 E-03$
$6.4 E-02$

$0.4 E-02$
$1.0 E-02$

6. $1 E-02$

$3.6 \mathrm{E}-03$

$1: 7 \mathrm{E}-02$

IE-OA

$7.3 E-05$
$6.4 E-05$

$5.7 E-02$
$1.6 E-02$

$1: 6 E-02$
$1: 0 E-03$

9. $0 E=05$

$2.3 E-0 S$
$: 9 E-03$

$1: 4 E-04$
$\because 0 E-05$

$3: 0 E-05$
$3.0 E-07$

1:2E-OS

$4.3 E=04$
$4.4 E-03$

C $3 E-0$

: $11 E-03$

i.iE-04

$1.3 E=06$
$5: 4 E-07$

$3.9 E-03$

(1)

$6.6 \mathrm{E}-07$

$7.4 E-03$

$45 E-03$

$1.6 E-04$
$1.2 E-05$

: $1 E-03$

: 6 EE-03

$\because \because 8 E-03$

G:TE-0

$1.6 E-01$
$6.9 E=02$

$\begin{array}{ll}6.9 E-02 & 4.0 E-02 \\ 1 . A E-01 & 2.3 E-02\end{array}$

TERUS
.2E-DJ

Y.3E=:0

J:SE-00

:OE-OS

$7.9 E-05$

$2.6 E-02$
$3.0 E-03$

$6.4 E-D 2$

:BE-OJ

$6.1 E-02$
$1 . B E-02$
$.6 E-O 2$

$6,1 E-02$

$: \forall E-02$
$: 9 E-03$

$0.0 E-02$
$1.7 E-02$
$.5 E-03$

I. $2 E-04$

.

$5.7 E-02$

STE-0J

$8.4 E-05$
$3.6 E-05$

$3.1 E-05$
$.5 E=03$

1.6E-0

$6.6 E-05$
$3.1 E-07$

$.6 E=06$
$.5 E=03$

$.2 E-04$
$4 E-03$

$\because 3 E-04$

IE

iIE-OA

$3.9 E-03$

1. $8 E-06$

S.TE -03
$. B E=03$
S

Y.SE-0 3

$7.2 E-04$

PE

$1.1 E-03$
$5.0 E-03$

$4.6 E-03$

$4.6 E-03$

5 
INHALATION DOSES AT I YEAR

(REMUMICROCURIE)

\begin{tabular}{|c|c|c|c|c|c|c|c|c|c|c|c|c|c|c|}
\hline $\begin{array}{l}N U C L I D E \\
N A=72 \\
D-32 \\
X-90 \\
4 N-5 A \\
Y N-56 \\
F E-55 \\
F E-59 \\
C O-57 \\
C O-58 \\
C O-60 \\
Z N-65 \\
R R-86 \\
S R-89 \\
S R-90 \\
S R-91 \\
Y-90 \\
Y-91 M \\
Y-91 \\
Z R-95 \\
Z R-97\end{array}$ & 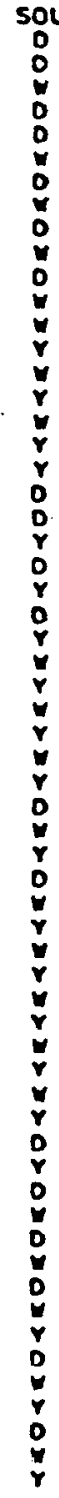 & 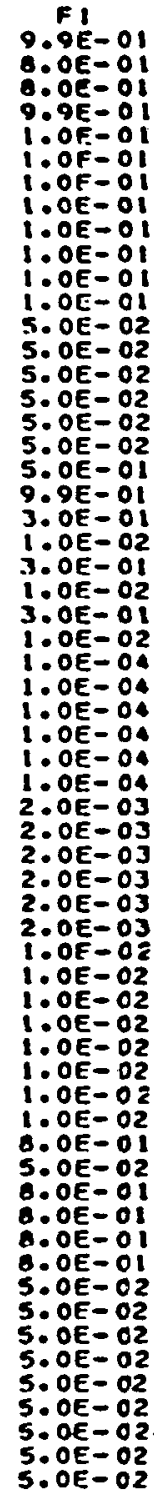 & 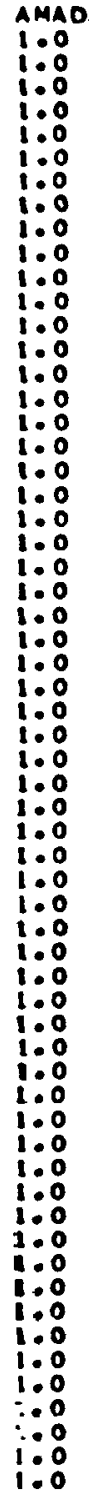 & 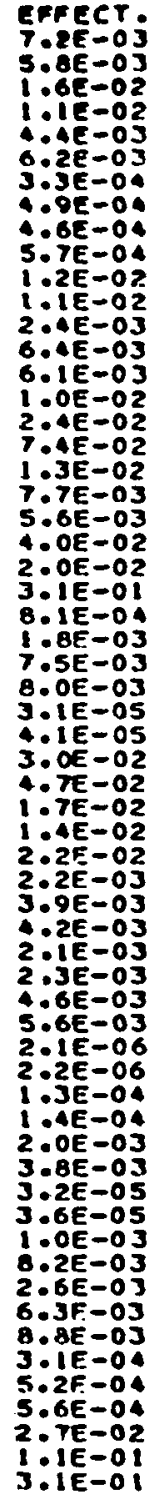 & 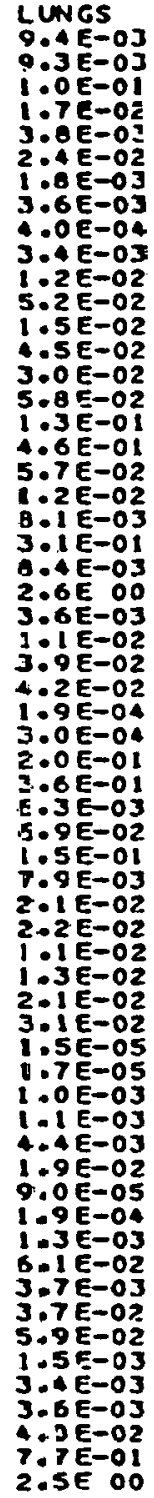 & 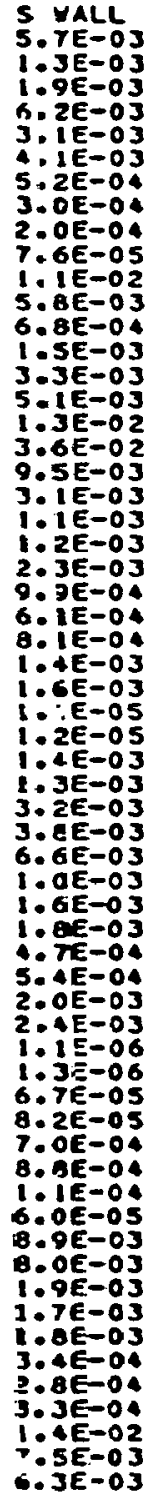 & 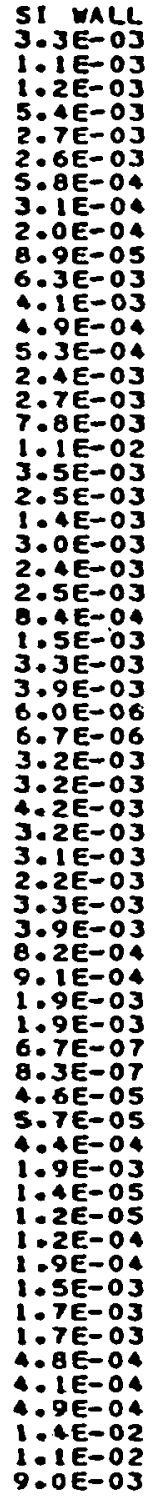 & 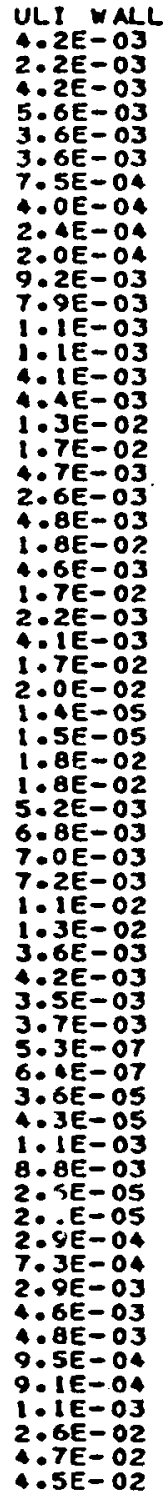 & 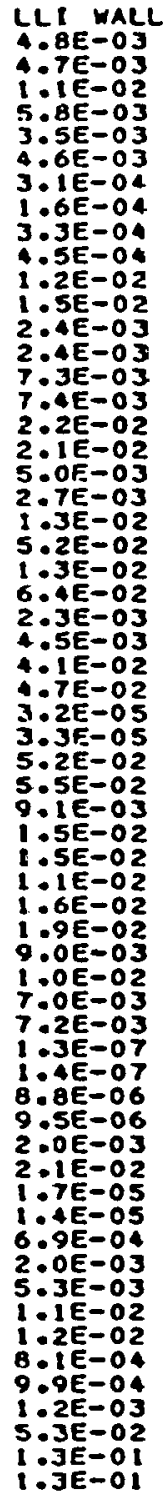 & 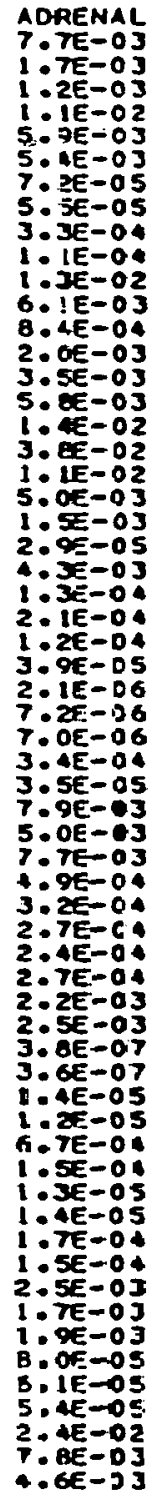 & 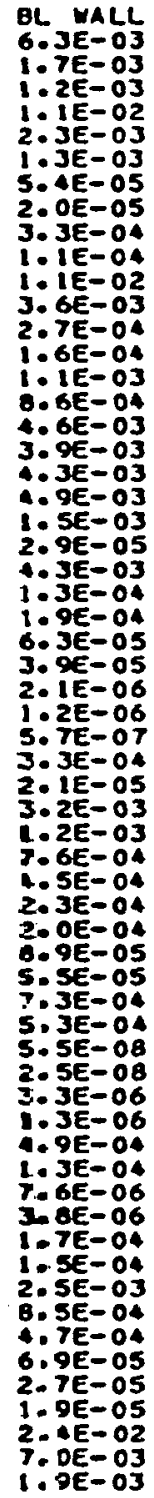 & 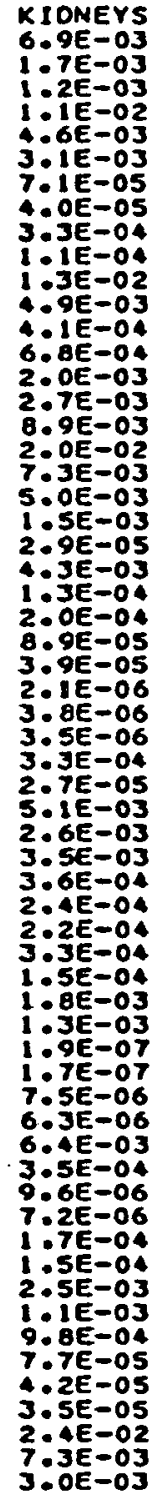 & 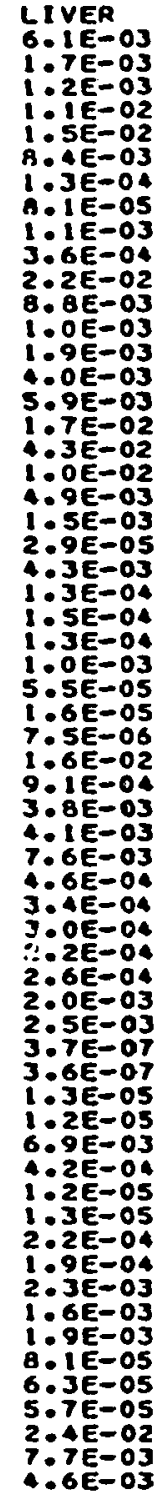 & $\begin{array}{l}51 \\
=03 \\
=0 \\
=03 \\
=03 \\
=0 \\
=0 \\
=0 \\
=03\end{array}$ \\
\hline
\end{tabular}




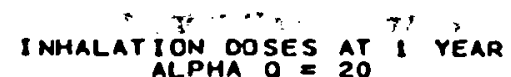

(REMIMICROCURIE)

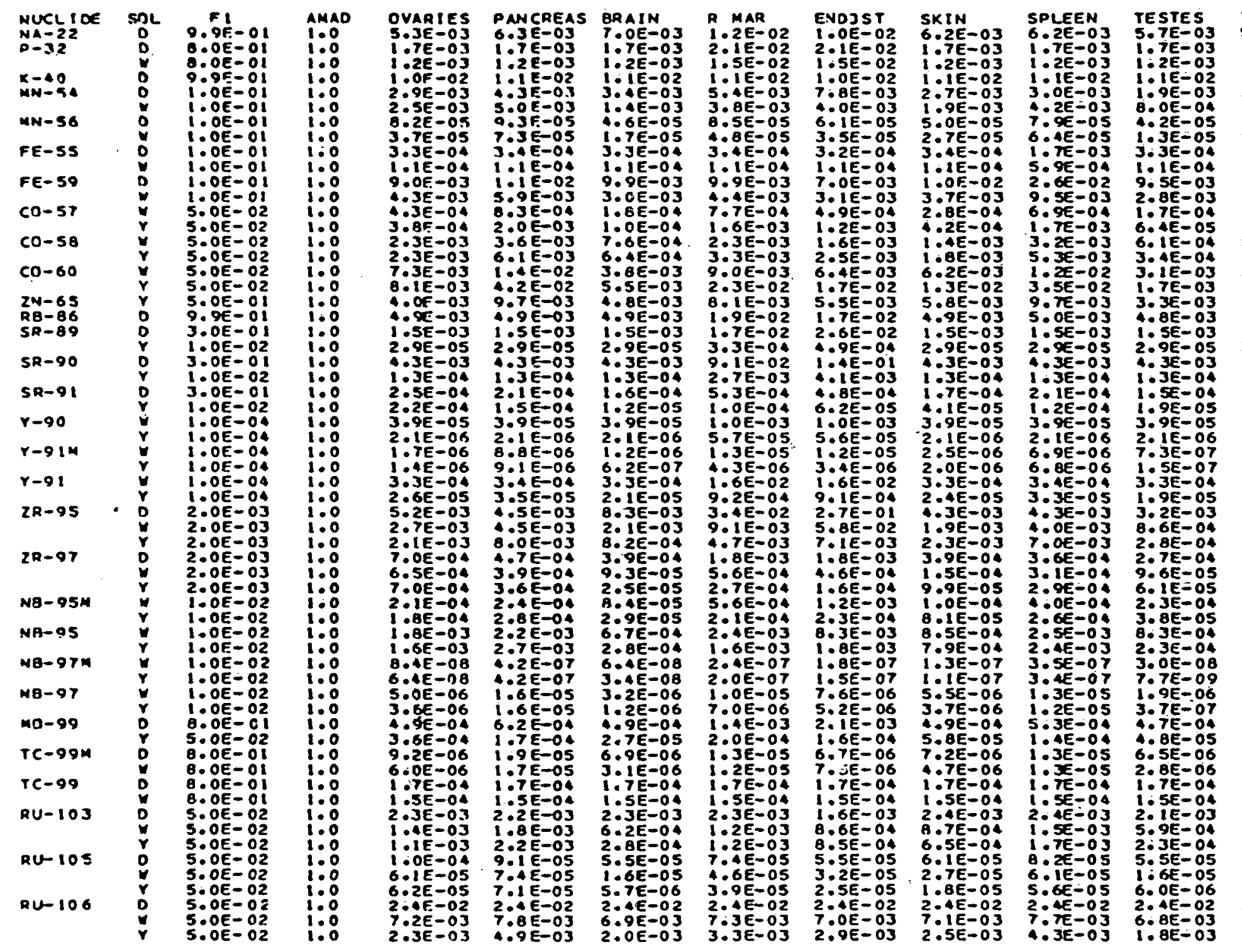

THYMUS THYAOTO UTERUS

$\begin{array}{lll}\text { S.SE-03 } & \text { 6.BE-03 S.IE-0J } \\ 1.7 E-03 & 1.7 E=03 & 1.7 E-03\end{array}$

I. $2 E-03$ 1.2E-03 $1.2 E-03$

$\begin{array}{lll}1.0 E-02 & 1.1 E-02 & 1.0 E-02 \\ 2.7 E-03 & 2.7 E-03 & 2.0 E-03\end{array}$

$6.6 E-03$ 2.8E-03 1.5E-0.

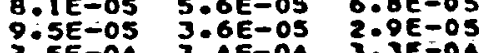

$1.2 E-04$ IE $1 E-04$ 1.1E -04

$9.4 E-03$ I.2E-02 9.1E-03

$\begin{array}{lll}7: 2 E-03 & 4 B E-03 & 3: A E-03 \\ 1: 1 E-03 & 4: 0 E-04 & 2: B E-04\end{array}$

$\begin{array}{lll}1: 1 E=03 & 7: 3 E-04 \quad 2: 2 E-04\end{array}$

$\begin{array}{lll}5.0 E-03 & 2: 1 E-03 & 1.2 E-03 \\ 0.2 E-03 & 3.2 E-03 & 1.1 E-03\end{array}$

2.2E-02 B.5E-03 $5: 1 E-03$

$7.6 E-02 \quad 2 \cdot 1 E-02 \quad 6: 2 E-03$

\begin{tabular}{lll}
$1.5 E-02$ & $8 .: A E-03$ & $3.8 E-03$ \\
\hdashline $.9 E-03$ & $5.0 E-03$ & $\because .9 E-03$
\end{tabular}

$\begin{array}{lll}\text { I:SE-OS } & 5.0 E-03 & 1: 9 E-03 \\ 3: 5 E-03 & 1: 5 E-03\end{array}$

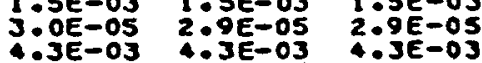

$1.3 E-041.3 E-04 \quad 1: 3 E-04$

$2: 1 E-041.8 E-04 \quad 200 E-04$

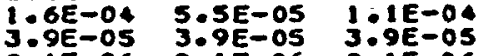

$2: 1 E=06 \quad 2: 1 E=06 \quad 2: 1 E=06$

$1: 0 E-05 \quad 3.5 E-06 \quad 1: 1 E=06$

$4.8 E-05 \quad 2.7 E-05 \quad 2.3 E-05$

$4.0 E-03$ i.2E-03 3.9E-0.

G.

$1.2 E-02 \quad 4.2 E-03$ 1.00-03

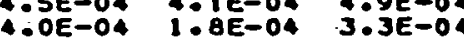

$\begin{array}{lll}3.6 E-04102 E-04 & 3.3 E-04\end{array}$

$3: 1 E-04$
$4: 3 E-04$
$3 E-04$

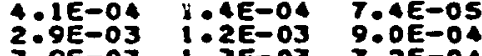

$3.9 E-03 \quad 1: 3 E-03$

$7.2 E-04$

$\begin{array}{lll}5.8 E-07 & 2.2 E-07 \\ 1 & 0.9 E-D E\end{array}$

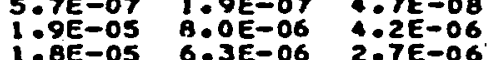

\begin{tabular}{lll}
$1.8 E-05$ & $6.3 E-06$ & $2.7 E-06$ \\
\hdashline $9 E-04$ & $4.4 E-00$ & $4.7 E=04$
\end{tabular}

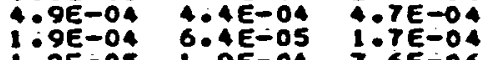

$1: 2 E-05 \quad 1: 9 E-04 \quad 7.6 E-06$

$1: 7 E=04$ A:SE-03 $1: T E=04$

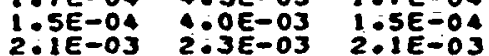

$\begin{array}{lll}2: 1 E-03 & 2: 3 E-03 & 2.1 E-03 \\ 2: 1 E-03 & 1: 1 E-03 & 8.3 E-04\end{array}$

2.6E-03 $9.7 E-0.04 \quad 5: 4 E-04$

$81 E-053: 5 E-05 \quad 3: 9 E-05$

$\begin{array}{lll}7.6 E-05 & 2.55 E=05 & 3 \\ 2 & 5 E-05 \\ 3.4 E-02 & 2.4 E-02 & 2.4 E-05\end{array}$

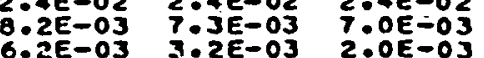


INHALATION DOSES AT I VEAR

(REM/MICROCURIE)

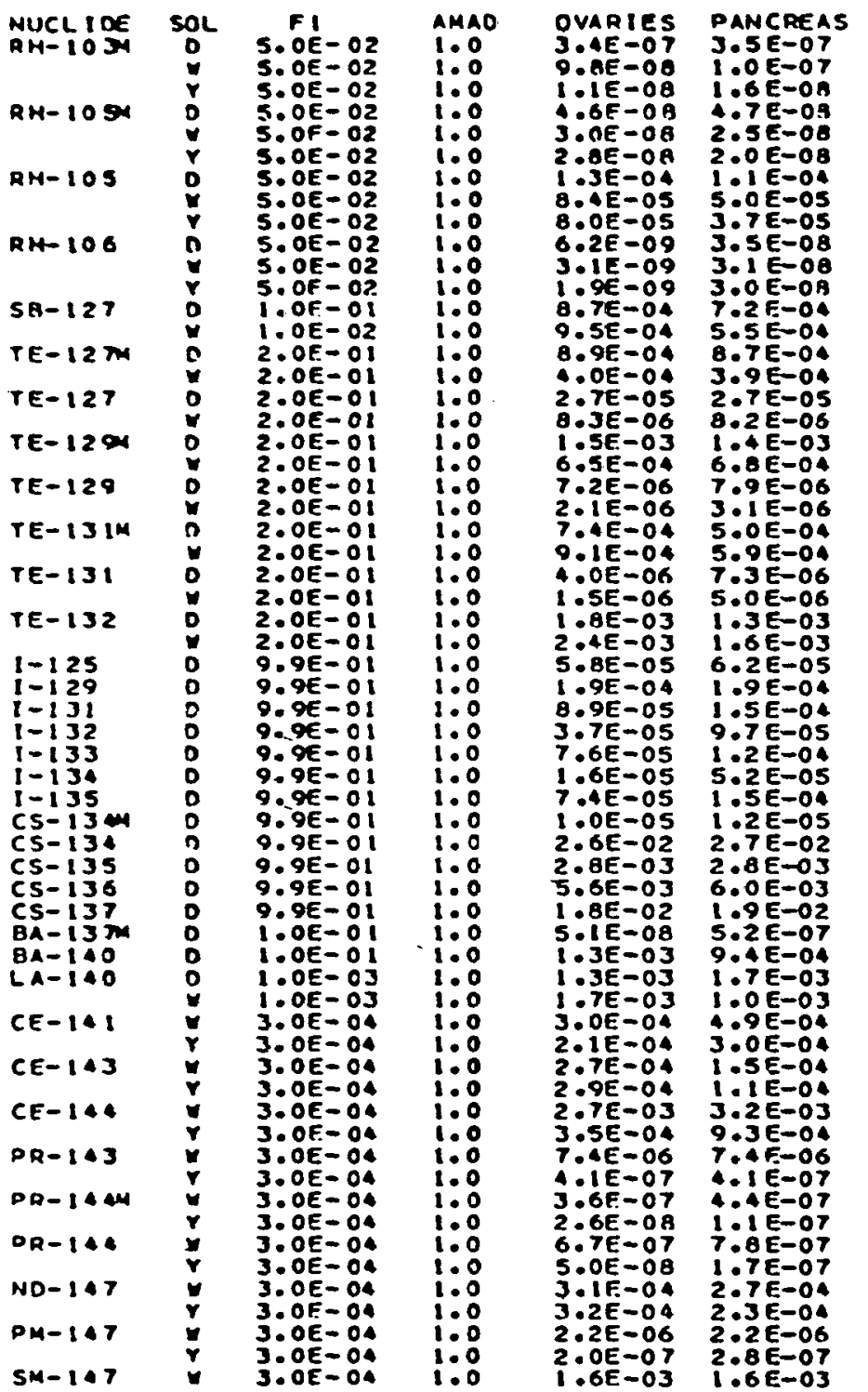

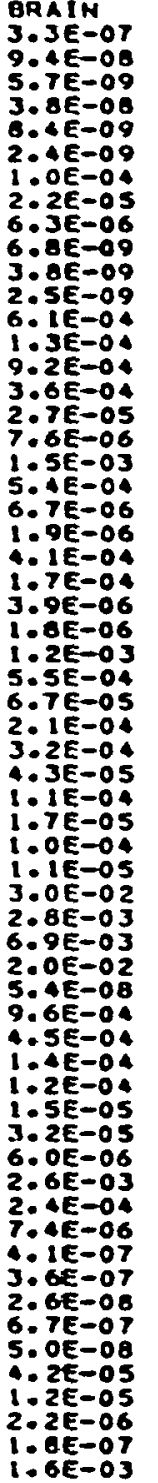

IET 07

2:O $5 E-O B$

i. IE-O4

3. $2 E-05$

$1.8 E-08$

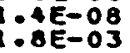

$6.2 E-04$
$3.2 E-02$

$1: 2 E-02$
$: B E=05$

$2.8 E-02$

$9.9 E-03$

$2.2 E-06$

$5.8 E-04$

$3.1 E-06$
$3.1 E$

$3.2 E-03$
$1.9 E=03$

$1.4 E-04$

$2.3 E-04$

$1.1 E-0$.
$2.5 E-05$

(1)

$2: 2 E-05$
$2.9 E-02$

$6.6 \mathrm{E}-03$

$1.8 E-02$
$2.2 E-07$

A: $\mathrm{AEE}-03$

$1.4 E-03$

$3.3 E-04$
$2.9 E-04$

$1.2 E-04$
$3.5 E-02$

$3: 1 E-03$

5.AE-05

B.OE-O

9. PE-OB

?.SE-03

$4.5 E-03$

$3.8 E-04$
$3.6 E$
SKIN

$3.3 E-07$
$3.0 E-07$ $9 E-08 \quad 3: 0 E-08$

$\because: B E-08$

$1.0 E-04$
$2.8 E-05$

$1: 3 E-05$
$1.2 E-08$

$9.1 E-09$
$7.8 E-09$

$6.2 E=04$

$2.2 E=04$
$B . T E=04$

3.SE-04

7.7E $: 05$

$5: 4 E-03$

$6.8 E-06$
$2.1 E-06$

$2: 1 E-06$
$4: 1 E-04$

$2.6 E-04$

$4.1 E=06$
$2.3 E-06$

I: $2 E-03$

$0.06-04$
$1.6 E-0.04$

$1.6 E-04$
$3.2 E=04$
$2.2 E-O 4$

$4: 9 E-05$

$2: 1 E-05$

$1.0 E-04$
$1: 1 E-05$
$3.1 E-02$

$2.8 E-03$
$7.1 E-03$

$2.0 \mathrm{E}=02$
$1.3 \mathrm{E}-0.07$

$1.3 E-07$
$8.0 E-0.04$

S. $6 E=04$

$1.6 E-04$

$6.7 E-05$
$5.3 E-05$

$2.9 E-05$
$2.7 E-03$

$2.7 E-03$
$3.8 E-0.04$

$3: 4 E=06$

:IE
$3.1 \mathrm{TE}-07$

$4.2 E-O B$

$6.9 E-07$
$7.5 \mathrm{E}-0 \mathrm{O}$

7.: $1 E-05$

$5.4 \varepsilon-05$
$2.2 \varepsilon-06$
2.06

$2.0 E-07$
$1.6 E-03$
3. $4 E-07$ TESTES 2.1E-07 9.5E-0B .4E-08 3. BE-08 $1: T E-08$ A. OE-O9

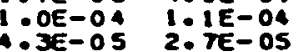
3.OE-05 $1: 1 E-05$ 2. $5 E=08$ 1. $1.6 E-09$ $6.7 E-04 \quad 5.8 E-04$ $06 E-04$
$6 E-7 E-04$ . OE-OA 3.4E-OA $2.7 E-05$

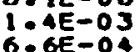
$\begin{array}{ll}3: 5 E-06 & 6: 6 E-06 \\ 2 E-06 & 1: 9 E-06\end{array}$ 2. $5.2 E-04 \quad 1.0 E-04$ $\begin{array}{lll}A E-0.06 & 1: 3 E=06 \\ 1: 3 E-03 & 1: 1 E-03\end{array}$ $1.5 E-03$ 5. $9 E-04$ $\begin{array}{ll}3: 3 E-05 & 5: 9 E-05 \\ 1.0 E E-O 4 & 1: 9 E-04\end{array}$ $3010-04$ 9. SE-05 1.2E-04 $7: 3 E-05$ $1.3 E-04$ 1.:ZE-05 $7: 7 E-05$ 2.6E-02

$2.0 E-03 \quad 6.0 E=03$

T. IE-OF

$3: 5 E=04$
$9: 2 E=-03$

$2: 2 E-03$

2. $4 E-0 B$

i: $:$ SE-:03 $1.0 E-05$
$2.0 E-05$ $4: 4 \varepsilon-05$ $2.8 E-012.6 E-03$ $2.1 E-02$

$\because 1 E=07$

$1.3 E=07$
$1.0 E-0,07$

$2: 1 E-04$
$3: A E-06$

औ. $1 E=07$

2. $1 E-0 B$

$1.6 E-07$

3. $9 E-08$

: $: 2 E-0.04 \quad 2.5 E-05$

$\begin{array}{lll}2: 3 E-07 & 2: 0 E=07 \\ 1: 6 E-03 & 1.6 E-03\end{array}$
THYMUS THYROIO UTERUS

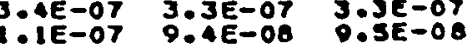
$1.9 E-08 \quad 5: 9 \mathrm{E}-09 \quad 7.5 E-09$

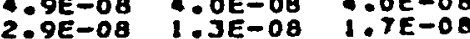
$2.4 E-08 \quad 6.9 E-09$ 1.3E-08 $1.0 E-04 \quad 1.0 E-04 \quad 1: 1 E-04$ $3.6 E-05$ 1:3E-05 3:JE-05 $4.4 E-08$ 1:5E-0B 2:5E-09 $4.3 E-08 \quad 1.4 E-08$ 1.JE-09 $6.4 E-046.4 E-04$ 6.TE-O4 $5.9 E-042.6 E-04 \quad 4.5 E-04$ $4.2 E-04$ 3.5E-04 3:3E-04 $2.7 E-05$ 2.7E-05 $2.7 E-05$ $1.4 E-03$ 1.5E-03 1.4E-03 $7.4 E-045.8 E-04 \quad 5.5 E-04$ $7.4 E-06 \quad 6 \cdot 9 E-06 \quad-7.0 E-06$ $504 E-06 \quad 2.3 E-06 \quad 2.0 E-06$ T.1E-04 5.6E-02 $4: T E-04$ $7.4 E-06 \quad 2.1 E-03 \quad 3: 0 E-06$

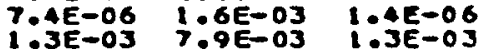
.9E-03 4.2E-03 1.2E-03

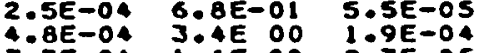
$7.3 E-04 \quad 1.1 E$ OO $8.7 E-O S$ $2.1 E-04 \quad 2: 7 E-01 \quad 3: 6 E-05$ $4.5 E-05 \quad 1.1 E-03 \quad 1.6 E-05$ 20105 T.SE-OS $\begin{array}{lll}1: 1 E-05 & 1.2 E-05 & 1.0 E-05 \\ 2: 7 E-02 & 3.5 E-02 & 2.0 E-02\end{array}$ $2.8 E-03 \quad 2.8 E-03 \quad 2.8 E-03$ $6.3 E-03 \quad 8.8 E-03 \quad 5.7 E-03$ $\begin{array}{lll}1.8 E-02 & 2.1 E-02 & 1.8 E-02 \\ 6.1 E-07 & 202 E-07 & 4.3 E-08\end{array}$ $7.7 E-04 \quad 8: 3 E-04$ B.:9E-04

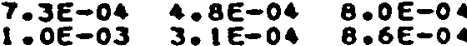
2E-DA 1.7E-OA 1.9E-O

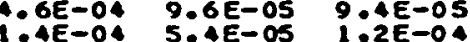
$1.1 E-04 \quad 3.0 E-05 \quad 1.2 E-04$ 3.0E-03 $2.6 E-03 \quad 2.6 E-03$

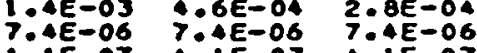
4 IE-07 $31 E-07$ A.IE-07 $4.9 E-07 \quad 3.8 E-07 \quad 3.6 E-07$

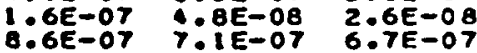
$2.5 E-07 \quad 9: 6 E-08 \quad 4.9 E-08$ 2.8E-04 $7.2 E-05$ 1:2E-0. $\begin{array}{lll}3: A E=07 & 2: 11 E-07 & 1: 9 E=07 \\ 1: 6 E-03 & 1: 6 E-03 & 1: 6 E-03\end{array}$ 
INHALATION DOSES AT I YEAR

(REM/MICROCURIE)

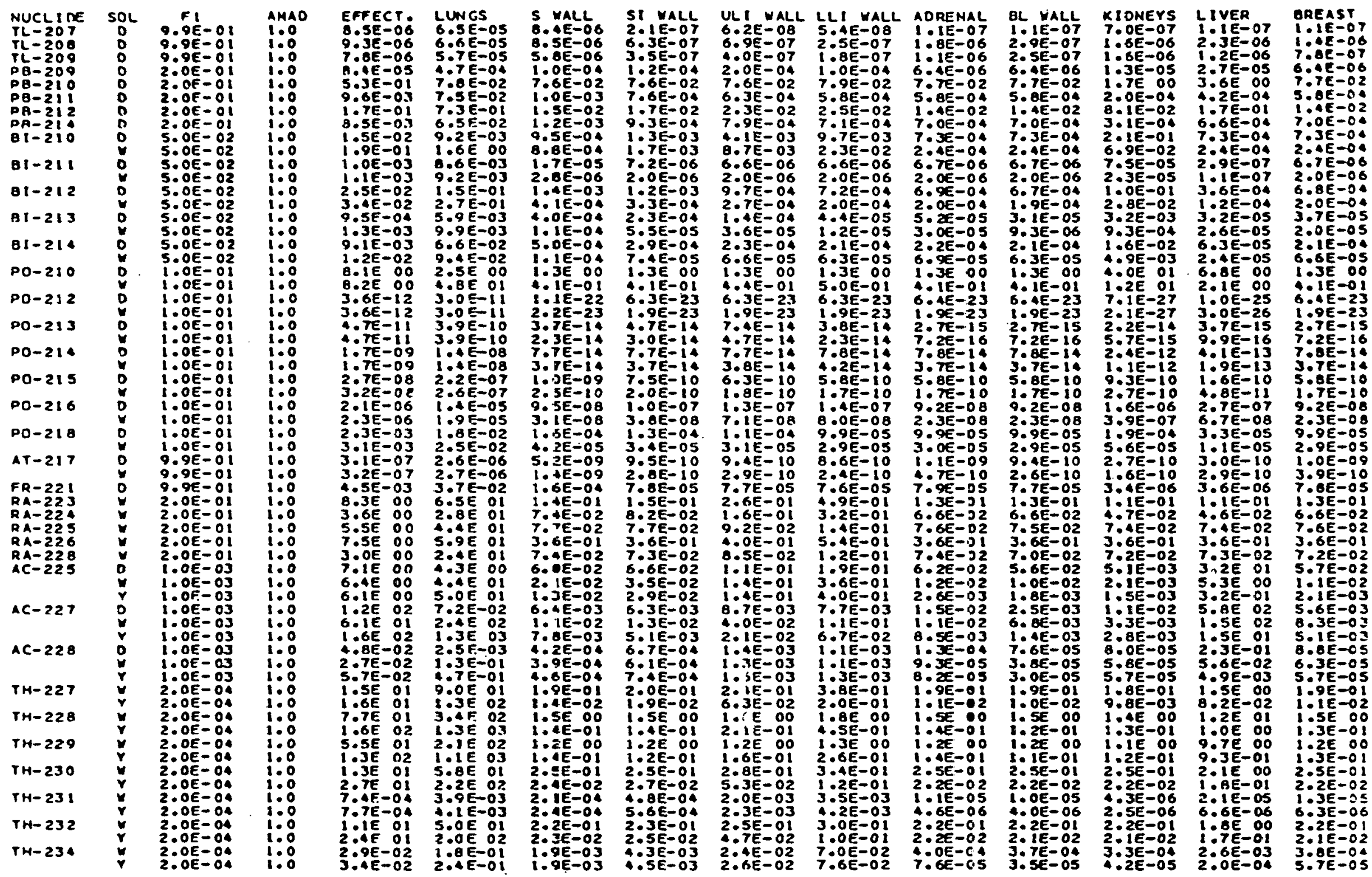


INHALATION OOSES AT I YEAR

(REMAMICROCURIE)

\begin{tabular}{|c|c|c|c|c|c|}
\hline 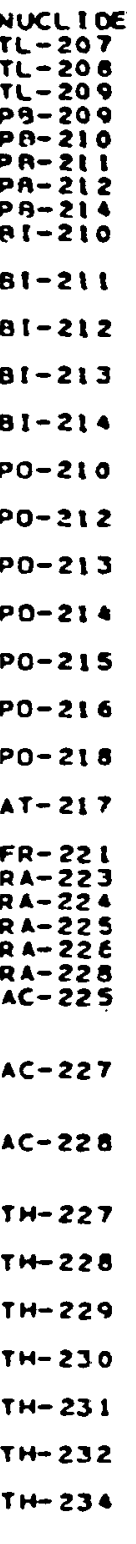 & 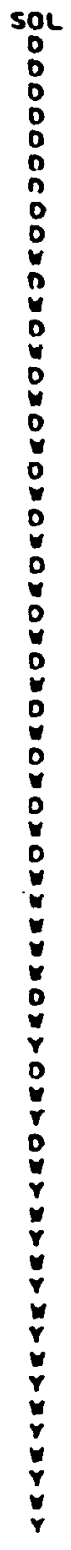 & 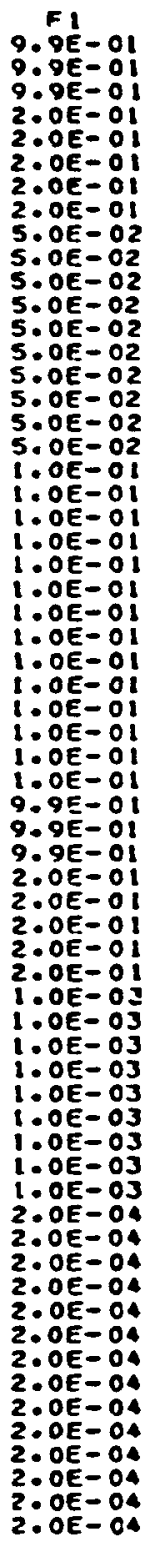 & 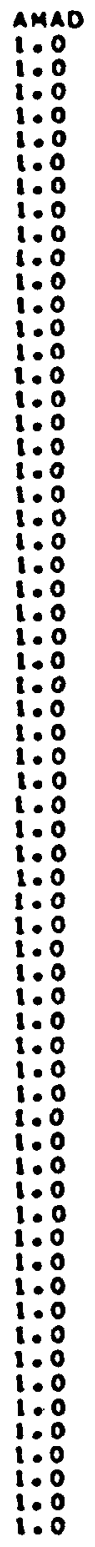 & 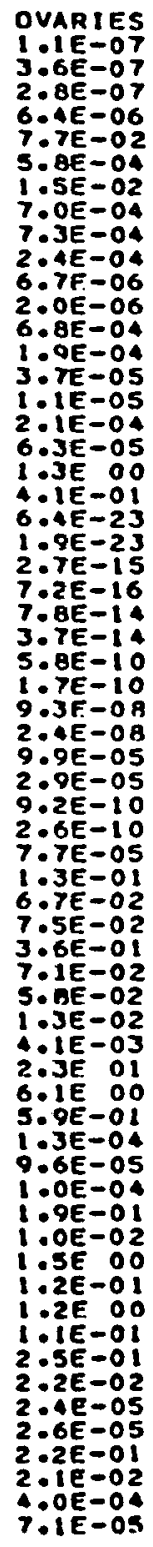 & 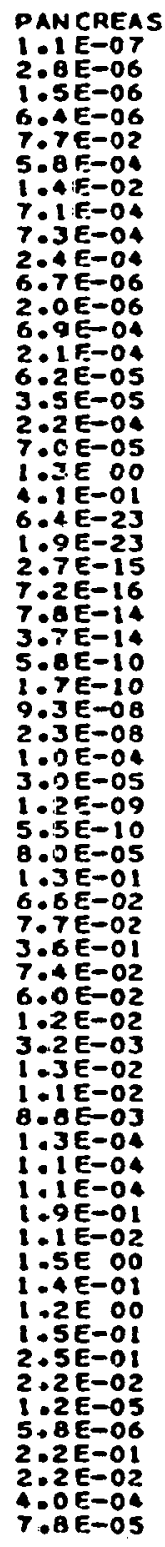 \\
\hline
\end{tabular}

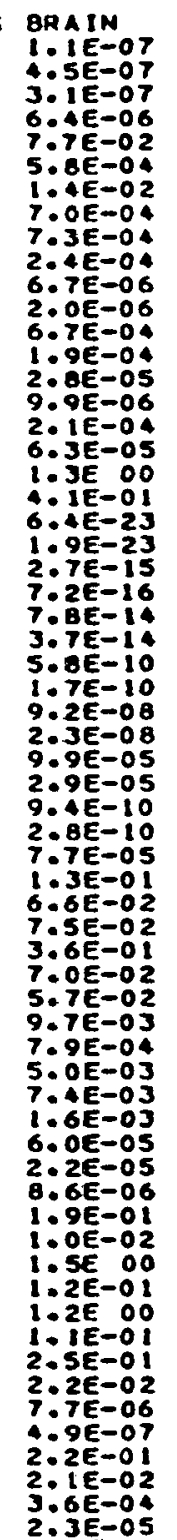

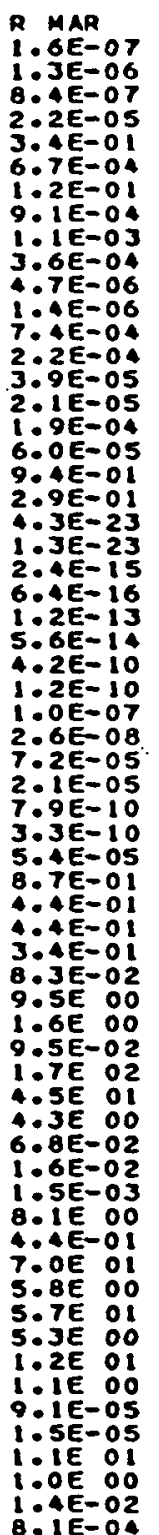

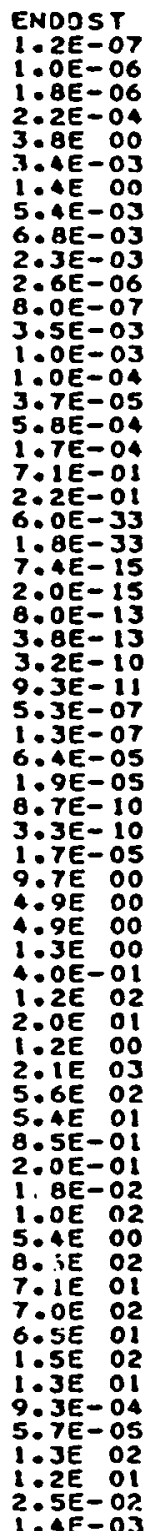

\begin{tabular}{|c|}
\hline 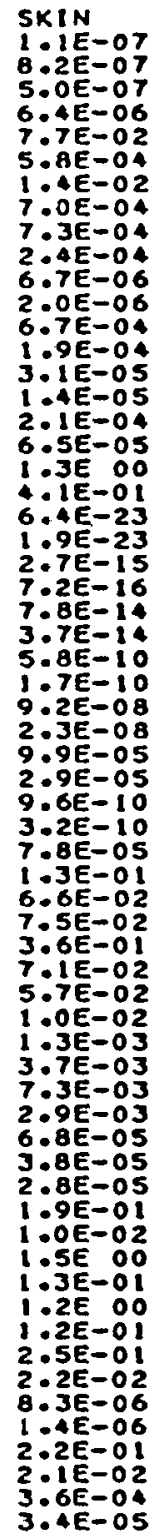 \\
\hline
\end{tabular}

SPLEEN SPLEE 1 1E- 07 2:AE-06 $7.76-0$
5 i. $9 E=06$ $3.96-03$
$2: 7 E-05$ $2.7 E-05$
$7.3 E-0.04$ $2: 4 E-094$
$2: 0 E-07$ 1:OE-O? $3.7 E-O A 4$
$1.2 E-O 4$ $4: 0 E-05$
$2: 3 E-05$ $6: 0 E-05$ $2.3 E-05$ 3 : IE 01 $1 \cdot 0 E-25$
$3.0 E-26$ $9.9 E-15$ i: $1 E-12$ I: $9 E-12$ 1: $\pi=10$ $2: 7 E-06$ 3. $2 E=04$ 9.6E-05 $2.6 E-10$
$4.2 E-06$ :.2E-06 $40 E-02$ $3: 06=01$ T.: $2 E-02$ 2. $1 E-03$ 3. $7 E-03$ $2: 2 E-03$ $6.4 E-03$
$6.00-05$ $8.0 E-05$
$8.0 E-05$ i:oE-01 $1: 0 E-02$ I:AE-O! I.: $1 E-O 00$ $2: 5 E-01$ 6. $6 E=06$ 5. $1 E-06$ $2.2 E-01$
$2.1 E-02$ 1. $2 \varepsilon-05$
TESTES $1.1 E-07$
$2.2 E-07$
$20-0.7$ 2.2E-07 T. $7 E-06$ S. $B E-04$ $1.0 \varepsilon-02$
$: 0 \varepsilon-0.04$ $7.3 E-04$ 2. $4 E-08$ $2.0 \varepsilon-06$ 6. TE-04 $1.9 E-04$
$2.0 E-05$ 8. $2 E-06$ 6. $3 E-05$ 1. JE OD $4.1 E-01$
$6.4 E-23$ 1.:PE-23 $2 E-16$
$7: B E-14$ 3. $7 E-14$ S.:BE-10 9. $2 E-08$ $9.8 E-05$ 2.9E-05 2. $6 E-10$ $1.3 E-01$ 6. $6 E-02$

7. $5 E-02$ 7. $0 E-02$ S.6EE-03 2. $3 E$ OI 6.1E DO S. $9 E-01$ $2.0 E-05$ i.9E- O1 1: $0 E-02$ 1. 2E-01 1.2E-O 2. $S E-01$ 2.9E-06 $2.2 E-01$ 3. $1 E-02$ 2. $3 E-05$
$1.1 E-07$

THYAOIO UTERUS

OEE-06 6.2E-06 JE-07 3.BE-07

6. $A E-06 \quad 6 . A E-06 \quad 6: 4 E-06$

S.AE-04 $5: a E-04$ S:AE-O.

$1 \cdot A E-02$ 1.AE-O2 $1 . A E-02$

$7.3 E-04 \quad 7: 3 E-04 \quad 7.3 E-04$

6.JE-06 6.7E-06 6.7E-0.6

$2.1 E-06 \quad 2.0 E-06 \quad 2.0 E-06$

2.2E

$40 E-05 \quad 3: 2 E-05 \quad 3: 6 E-05$

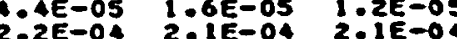

$7.4 E-05$ 6.6E-05 6.3E-05

$1.3 E 00$ 1.JE DO $1: 3 E 00$

$6: 4 E-23 \quad 0: 4 E-23 \quad 6: A E-23$

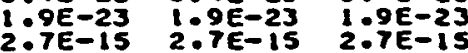

$\begin{array}{lll}7.2 E-16 & 7: 2 E-16 & 7.2 E-16 \\ 7: B E-14 & 7: 0 E-14 & 7: 8 E-14\end{array}$

3.7E-14 3.TE-14 $3.7 E-14$

$1.7 E-10$ 1:PE-10 1:7E-10

$2.3 E-08 \quad 2.3 E-08 \quad 2.3 E-08$

$9.9 E-05 \quad 9.9 E-05$ 9.9E-05

3.0E-05 $2.9 E-05$ 2.9E-0S

1. $8 E-10$
$6.5 E-10$

T.8E-05

$6.6 E-02$
$7.6 E-02$

3.6E-01 3.6E-01 3.6E-0

$7.0 E-02 \quad 7.2 E-02 \quad 7.0 E-02$

$1: 3 E-02 \quad: 00-02$ i: $: 1 E-02$

$4.0 E-03$ J.JE-03 3.1E-03

$9.2 E-03 \quad 7.4 E-03 \quad 6.9 E-03$

$1.2 E-02 \quad 3.9 E-03 \quad 1.5 E-03$

$1: 2 E-04$ ?:OE-05 $6.2 E-05$

$1: 2 E-04$
$1.3 E-04$
$0.9 E-05$

$\because 9 E-01 \quad 1: 9 E-01$
$1: 9 E-02$

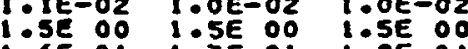

I. $6 E-01 \quad 1.3 E-01$ 1.2E-0I

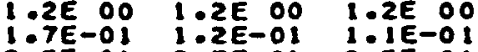

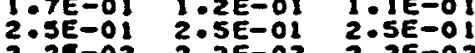

$2.2 E-02 \quad 2.2 E-02 \quad 2.2 E=0$

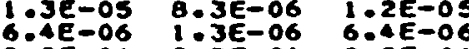

$2.2 E-01 \quad 2.2 E-01 \quad 2.2 E-01$

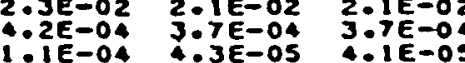


(REMIMICROCURIE)

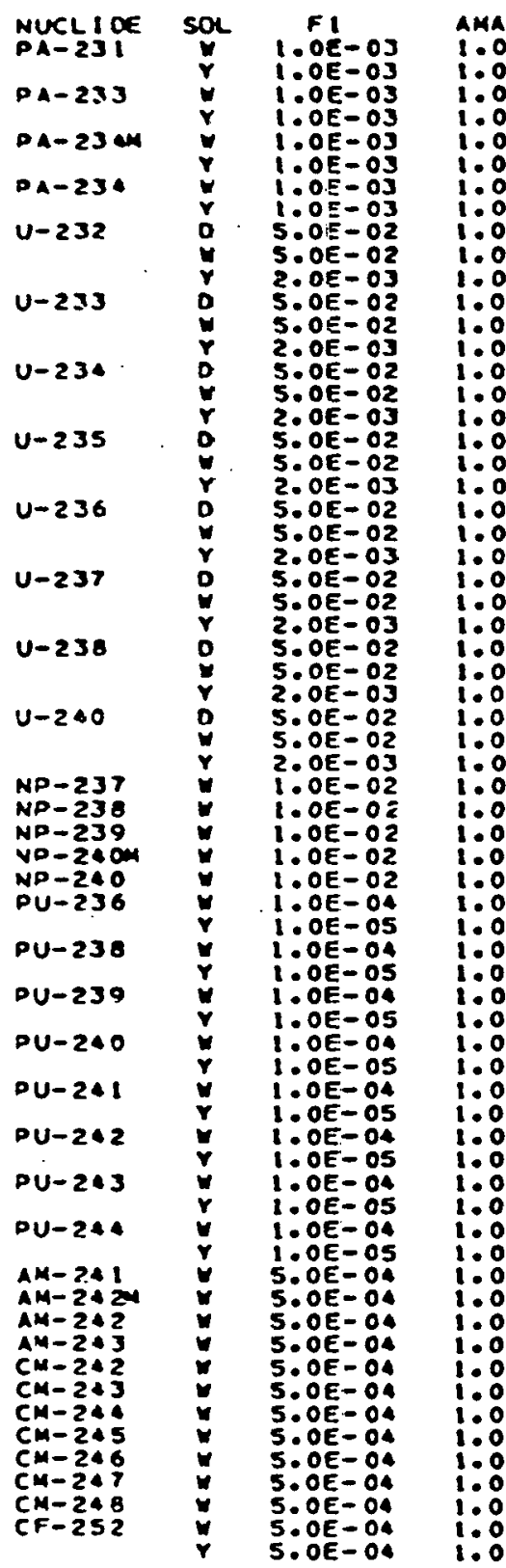

EFFECT: LUNGS

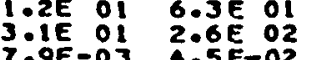
Q.:EE -03 $6.45-02$ 3. $B E-06 \quad 3: 1 E-05$ $\begin{array}{lll}8.6 E-04 & 5: 1 E-03 \\ 9.3 E-0.4 & 5: 5 E-03\end{array}$ $9.6 E-01$ 1.3E 00 P.IE OI $9.0 E$ OI $7.9 E-01$ 1.2E OO $7.3 E$ OO $5.9 E$ OI 7.BE-OI 1.22 OO 7.2E O० S.9E OI $7.3 E-O 1$ 1.1E $O 0$ 2.5E OI $2: 1 E$ Oz $\begin{array}{lll}7.4 E-01 & 1.1 E & 00 \\ 6.8 E & 00 & 5.5 E \text { OI }\end{array}$ 2.6E $011 \cdot 2 \cdot 1 E 02$ $\begin{array}{ll}3.2 E-03 & 1: 7 E-02 \\ 3.4 E-03 & 1.9 E-02\end{array}$ $6.5 E-01$ 1.OE OO

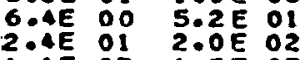
: $1 E=03$ A $: 5 E-0.3$ $2: 0 E-03 \quad 1.2 E-02$ $1.3 E 01 \quad 5.9 E-01$ $\begin{array}{ll}3.2 E-03 & 1.5 E-02 \\ 2 & -2 E-02\end{array}$

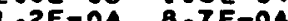
1.AE 01 6.8E OI 3
$1: O E$

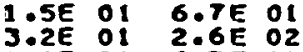

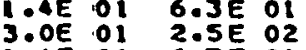
$1: A E$ OI $6: 3 E$ OI $3.0 E$ OI $2.5 E$ OZ $\begin{array}{ll}7.9 E-03 & 2.5 E-02 \\ 2.6 E-02 & 2.1 E-01\end{array}$ $1.3 E$ OI $\begin{array}{lll}2 \cdot B E & 01 \\ 1 . T E & -04 \\ 1.0 E-O A\end{array}$ $\begin{array}{ll}6.0 E & 01 \\ 2.3 E & 02 \\ 1.3 E & -03\end{array}$

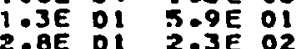
1.SE DI $6.7 E$ OI 3.TE-ว? $1.5 E$
$A .9 E-O I$ OIE JI $5.5 E$ OI

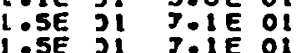
IAE Ji S.6E OI

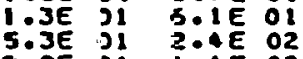
G.PE DI
5 YALL SI YALL ULI WALL LLI WALL AOREVAL BL YALL 6.2E-D3 9.6E-03 3.7E-02 $1.1 E-01$ $1.0 E-03$ 3.TE-03

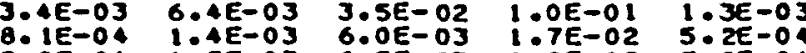
(1.5E-03 $6.5 E-03$ L

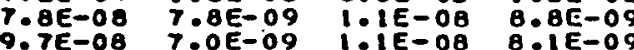
.5E-04 $7.0 E-09$ 1.IE-OB 6.5E-O4 . $8 E-02$ 2 0 (OE-02 $2.2 E-03$ 2.SE-02 $2.9 E-02 \quad 5.8 E-02$ 5. TE-02 S.BE-OZ B.SE-O $\begin{array}{lll}5.9 E-02 & 2: 2 E-02 & 4.5 E-02 \\ 1.5 E-03 & 6: 7 E-03 & 3.3 E-02\end{array}$ S. $6 E-02$ 5.7E-02 $6.55 E-02$ $3: 9 E-03 \quad 6.7 E-03 \quad 3.2 E-02$ $5.4 E-02$ 5:5E-02 $6: 3 E-02$ $1.9 E-02 \quad 2.1 E-02 \quad 4.8 E-02$ $5.3 E-02 \quad 5.4 E-02 \quad 6: 1 E-02$ $1.8 E-02$ 3. (1) $5.0 E-04 \quad 1.0 E-03 \quad 4.7 E-03$ $\begin{array}{lll}5.52 & 1.2 E-03 & 5.6 E-03\end{array}$ $1.7 E-0.02$
3 -1
5 $5.7 E-04$
$9.8 E-04$ 9.2E-04 $1.8 E-03$ 6.0E-03 (a) $5.0 E-04$ $2.6 E-06$ 2.6E-03 $1.0 E-02$ $\begin{array}{ll}6.4 E=03 & 1.0 E-02 \\ 2.8 E-03 & 6.5 E-03\end{array}$ $6.0 E-03$
$2.0 E-03$ 6. $0 E-03$ $2.7 E-03$ 1. $3 E-05$ 5. $7 E-03$ $2.5 E-03$
$3.5 E-05$ $1.0 E-04$
$1.0 E-02$ $1: 5 E=02$ $6.7 \in=-03$
$9: 0-0.04$ $0.4 E-03 \quad 1.2 E-02$ $.2 E-03$ (O) $8: T E-03$ i: $1 E-02$

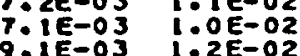
(3) 2: $3 \mathrm{E}-01$ $\begin{array}{lll}1.3 E-01 & 100 E-01 & 1: B E-01 \\ 2.3 E-01 & \end{array}$

B. $4 E-09$ C.AE- 09

9.0. a

$1.1 E-01$
$9, T E=02$

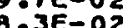

$8.03 E-02$
$1: 1 E-01$

$9.7 E-02$
$9.3 \mathrm{E}-02$

$1,1 E-01$

$7: 9 \mathrm{E}-\mathrm{O} 2 \mathrm{O}$ $1.0 \varepsilon-01$ $5.0 \mathrm{E}-03$ $1.2 E-02$ $1.5 E=02$ $1,1 F-01$ $4.5 E-03$ S.OE-03

$1: 1 E-0$

5.2E-03 l:1E-02

$4.9 E-03$

$3.1 E-05$ $1: 1 E-02$
$9: 1 E-006$ B. $0 E-06$

$3.8 E-02$

$1: 2 E-01$
$1: 1 E-01$
$1: 1 E-01$ i. $1 E-01$ $9.4 E-03 \quad 3: 7 E-02$ $9: 5 E-03$

$3.0 E-05$
$3.0 E-05$

$1: 7 E-04$ $1.0 E-01$ $1.0 E-01$ $1.00-01$ $5.2 E-04$
$5.3 E-04$ $5.3 E-04$ $1.0 E-01$
$9: 8 E-02$ $1.9 E-04$ $1.8 E-01$ :7E-OI 1:1E-01 $2.4 E-03$ T.2E-01 i. $3 E-01$ i.2E-01 1 $11 E-01$ 6.9E-: $3.5 E-0 !$
$3.4 E-0 !$ 3. $6 E-04$ 3. $5 E-04$ I: $7 \in-09$ OP $9 E-1$ $2.2 E-09$ A. 5E-09 $1.0 E-03$ $4 \cdot 1 E-03$ (

3.6E-03 $3.0 E-03$ $3.3 E-04$ 3. $3 E-04$ $3.4 E=04$ 3. $3 E-04$ $\begin{array}{ll}1 \cdot 1 E=06 & 8.6 E-07\end{array}$ $\begin{array}{ll}3.2 E=03 & 2.1 E=07 \\ 3 & 5 E-03\end{array}$ 3.: $5 E=04$

2. $2 \varepsilon=06$ $3.1 E-04$
$2.2 E-06$

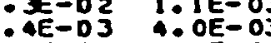

$6.9 E-D$ A.JE-O

$7.5 E-0 J 4.2 E-03$

6.: $3 E-J 3 \quad \because 5 E-03$ S.AE-JJ $\because: 1 E-0 J$ $\begin{aligned} & 5.2 E-J 3 \\ & 9.2 E-33\end{aligned}: \because E E-03$ 4.TE-ग T.OE-O $\begin{array}{ll}2.0 E-D 1 & 3.2 E-02 \\ 2.4 E-01 & 1.5 E-02\end{array}$
$2.3 E 00 \quad 1.0 E 00$ $1.6 E-03 \quad 1.7 E=03$ $2.0 E-09$ A. $3 E-09$ $1.2 E-04$ 1.BE-04 $3.5 E$ DO $1 . B E-02$ 3.7E-01 $5.6 E-03$ $8.6 E 00$ 5.7E-O2 2.0E-01 I:JEE-OJ $5.6 E=02$ $2.0 E-01$ (OE 7.9E $00 \quad 3.9 E-02$ $2.3 E$ 1.3 B.IE OO 5.3E-O $2.4 E 001.6 E-02$ $1.6 E-04$
$7.5 E$ $1.5 E-04$ $1.5 E=04$ I.AE-OA $2.2 E 00 \quad 1: 1 E-02$ $2: 7 E-03 \quad 4.9 E-05$ 5.3E-0S 6.9E-OS $1: 9 E-04$ 6.9E-05 5. JE- 05

$2.8 E-01$
$3.2 E-03$ $9.6 E=0$. 2:5E-0S

$\begin{array}{ll}1.2 E-05 & 2: 9 E \\ 6.2 E-06 & 200\end{array}$

$4.0 E-03$ $3.7 E-03$ 3. $7 \in=03$ 3. IE OI $2.9 E$ : $2.5 E$ :0

$1.0 E-06$

$3: 6 E-07$

$3.6 E-03$
$3.2 E-0.04$

$2.2 E-0$

2.TE OI $2.8 E-06$ 2.5E-05 $6.6 E-03 \quad 2.7 E$ O

$5.3 E=03$

greast

OPe-0. $3.5 E-0$. 2: $1.2 \epsilon=0$. $6.7 E-0 z$ $4.6 E-03$ i: $\mathcal{i}-02$ i. $: j E-03$ $1.6 E-02$ $1.3 E-03$ 1:6e-02 $3 \cdot A E-03$ $1: 6 \varepsilon-02$ $103 E-0.00$ 1.1E $=04$

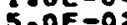
$105 E=02$ $3.7 E-05$

I:TE $=0$.

$6.0 E-05$

I. 4 E-OS

$\because 1 E-03$

$30 E-03$
$3.0 E-0.03$

3. $B E=04$

3.7E $=03$

$3.7 E-03$

$3.6 \mathrm{E}-0$
3

5.9E-07 3.6E- $=04$ 作 $8.2 E-03$ 2.4E DO Q.4E-OJ J:LE DI $5: 7 E=0$ $1.2 E-05$ 6.6E-O2 $1.3 E-05$ $3.9 E-03$ I.OE O1 $3.4 E-03$ $5.6 E-03 \quad 3.2 E$ of $5.1 E-03$ $4: 9 E-03$ J:OE O1 $\because .6 E-03$

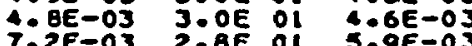
3.2E-01 $1.1 E$ O2 $2 . A E-01$

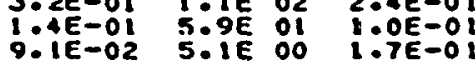




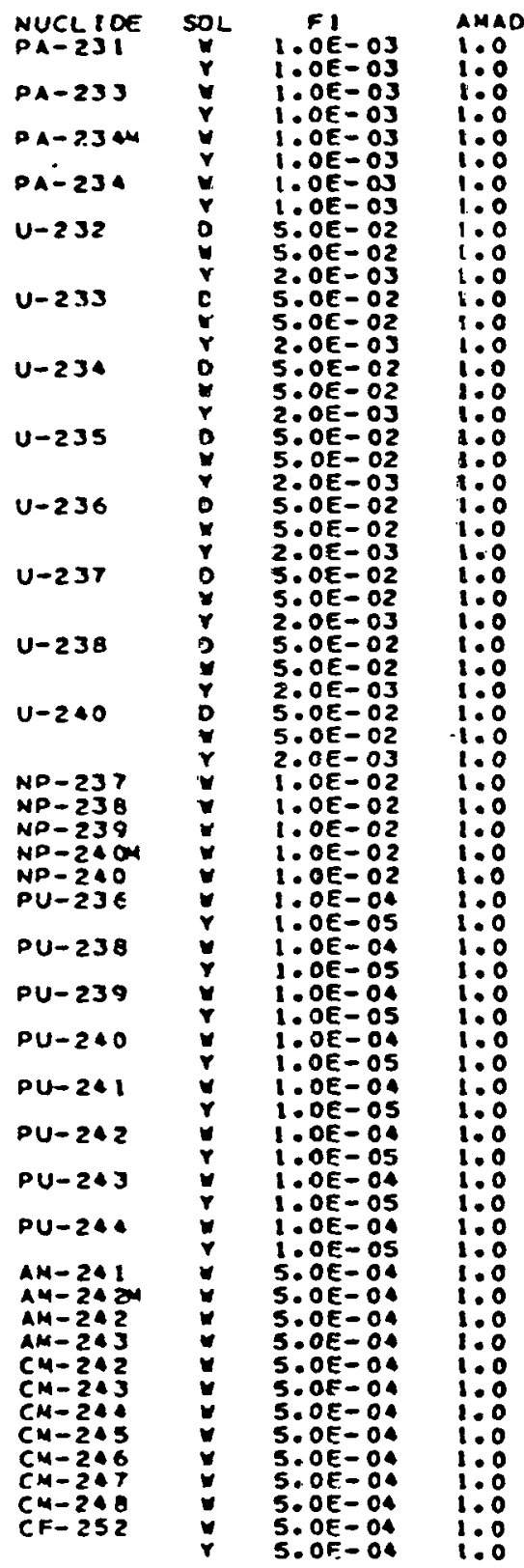

OVARIES PANCFEAS BRAIN $1.9 E-0.04$ $\because 9 E-04$
$\triangle 9 E-04$ S.:OE $1.6 E-09$ $2.1 E=04$
$2 . A E=04$ $6.4 E=02$
2

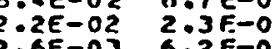
$5.6 E-02 \quad 5.2 E-0 J$ $1.7 E-02$ ? $1.7 E-02$ $5.6 E-02$ 5. $1: .6 E-02 \quad 1: 6 E-02$ $5.4 E-02 \quad 5.4 E-02$ $\begin{array}{ll}1.6 E=02 & 1: 7 E-02 \\ 1.9 E-03 & 4.9 E-03\end{array}$ $5.3 E-02 \quad 5.3 E-02$ $1.6 E-02$ $1,2 E-03 \quad 1.2 E-03$ 2:BE-OA 1:TET:

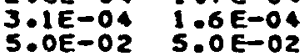
$1.5 E-02$ 1.5E-02 I.ZE $9.1 E-05 \quad 7.7 E-05$ $1.1 E-046.5 E-05$

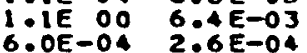
$\begin{array}{ll}2 . A E-04 & 1.1 E-04 \\ 6.4 E-00 & 3.2 E=07\end{array}$ $\begin{array}{ll}6.4 E-08 & 8.2 E-07 \\ 5.7 E-0.6 & 2.5 E-05\end{array}$ $1.2 E$ OC $4: 1 E-03$ $1.2 E$ OC $4.0 E-03$

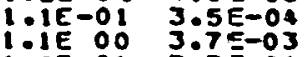
$1: 0 E-01$ 3. $3 \mathrm{E}-04$ $1.2 E-0.023$ $9.0 E-04$
$1.2 E-04$

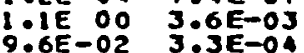
$3.2 E-06 \quad 4.3 E-06$ ( $0,7 E-02$ I.2E-02 $1.2 E 00 \quad 4: 4 E-O 3$ $\begin{array}{ll}4.9 E-01 & 6.4 E-02 \\ 2 . S E-03 & 1.4 E-05\end{array}$ $\begin{array}{ll}2.6 F-03 & 1: 4 E-O 5 \\ 1.2 E & 00\end{array}$

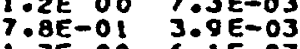
1:3E DO $6.1 E-03$ :ZE OO 5 O. $3 E-03$

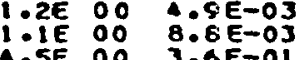
$2.5 E$
$2.4 E$
2

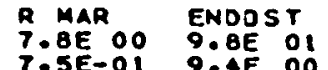
\begin{tabular}{lll}
$3.0 E-03$ \\
\hdashline$: 1 E=0.0$
\end{tabular} i. IE-0.4 $2.5 E-03 \quad 2.1 E-02$ 1.5E-O4 $\begin{array}{lll}5.5 E-09 & 1.5 E-0 E & 1.5 E-09 \\ 3.0 E-09 & 5 E-09\end{array}$ 2.6E-09 $2.0 E-09$ 1.6E-04 2.7E-04 6.JE-OS 2.9E-03 1:AE-DS $2.3 E-0$ 5. $7 E-02$

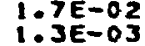
$5.6 E-02$
$1.6 E-0.02$ 2.7E-O1 3.0E-OS $\begin{array}{ll}8.9 E-02 & 1.2 E-00 \\ 1.1 E-02 & 1: 2 E-01\end{array}$ 5.5E-02 7.2E-01 5.7E-02 $\because \because A E-03 \quad 5.7 E-02$ $1.9 E-01$ 2.4E 0O S.6E-O2 $\begin{array}{lll}5.5 E-02 & 7.1 E-01 & 1: 6 E-02 \\ 4: 4 E-03 & 5.6 E-02 & 1: 3 E-03\end{array}$

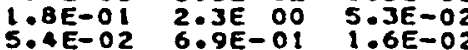
S: $1.6 E-02$ $1 . A E-0.03$
$5.3 E-02$ $1.0 E-02$
$1.2 E-03$ $5.4 E-02$ 1. $9 E-01$ $5.2 E-02$

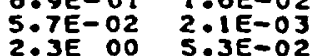
6.TE-01 1.6E-02 S. (.1E-03 5. $2 E-06$

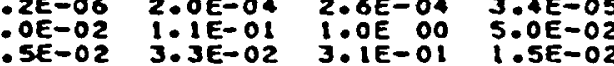
$1.2 E=03$
$0.7 E-05$ $4: 7 \varepsilon-06$

$: 3 E-03$
$: Z E-05$

$1.5 E-05$ $1.8 E-07$
$4.1 E=06$ 3.: $1 E-03$ 3. OEE 03 $3.7 E-03$
$3.3 E-04$ 3. $7 \in-03$ $3.3 E-O A$
$9.0 E-0.7$ 2.2EE-07

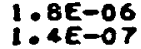
(.:0E-03 $1.1 E-03$
$: 6 E-004$ $1.0 E-05$ $4.6 E-03$
$3.0 E-03$ $4.6 E-03$ $\because: 2 E-03$ $4.3 E-03$
$4.0 E-03$ $1.36-01$
$5.6 E-02$
$2.0 E-02$ $3.3 E-02$ 3.IE-O $3.4 E-04 \quad 9.3 E-04$ A. $6 E-05$ 2.3E- 3

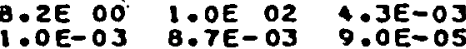
$3.6 E-04 \quad 2.6 E-03$ $1.5 E-05$ 1.AE-0S $8.6 E$ OO $1.1 E$ O2

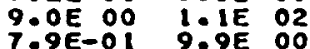

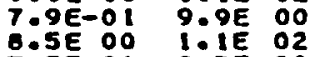
B.5E-01 9.3E OO

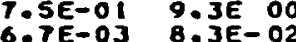
. $9 \varepsilon-04$ 8.1E 0O 1:0E O2 i. IE-05 $8.0 E 00$ $8.9 \mathrm{E}$ DO $8.5 E-05$
$6.5 E-06$ $9.9 E$ O1 $9.1 E 00$ $\begin{array}{ll}3.6 E & 00 \\ 2.0 E-02 & \end{array}$ $2.0 E-02$
$8.0 E-00$ $9.5 E$ OO $1: 1$ E 02 $2.4 E-01$ T.6E-OL $1: 1 E$ O2 $4: 6 E-03$ $\begin{array}{ll}7.2 E & 01 \\ 1.2 E & 02\end{array}$ 9.OE OO $1: 1 E$ OL $\quad 4: 2 E-0 J$

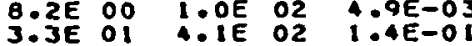

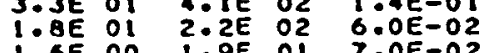

SPLEEN B:SE-O 5. $3 E-04$ 4. ZE-O $1.7 \mathrm{-04}$ 1. TE-OA 5. $1 E-02$ $5.4 E-03$ $1: 7 \mathbb{R}^{2}-025$ $1.3 \mathrm{~B}-03$ $1.0 E-02$ $1.3 E-03$ $1 . T E-02$

$5.7 E-03$

$1: 6 e-02$

I:ZE-03 i: $5.0 E-02$

1: $5 E-02$

6:SE-OS

$5: 11 E-05$

$2.2 E-04$
$8.5 E-05$

B. 6 BE-07 $8.1 E-06$ $\because 11 E-03$
$: 5 E-04$ $4.0 E-03$ 3.7E-0. $3.7 E-03$ $3.3 E-0.7$
$3.9 E-07$ 3.0E-07 $3.5 E=03$ $2.1 E=06$ $6.1 E-03$ $2.2 E-05$ $2.3 E-05$ 3. $\mathrm{TE}=04$ 3. $\frac{T E-03}{3 E-04}$ 3. TE-OJ $9: 4 E-07$

$6.8 E-07$

$3.6 E-03$
$3.4 E-0.0$ 2. TE-06

$1: 1 \in=02$

$5.2 E-03$

$\begin{array}{ll}1.3 E-05 & 2.6 E-03 \\ 5.6 E-03 & 1.2 E\end{array}$ $\begin{array}{ll}3.9 E-03 & Y: A E-01 \\ 5.2 E-03 & 1: 3 E\end{array}$ \begin{tabular}{lll}
$2 E-03$ & $1: 3 E$ & 00 \\
\hdashline $6 E-03$ & $1: 2 E$ & 00
\end{tabular} AE
$6.3 E-03$ $\begin{array}{lll}6.3 E-03 & 1: 1 E & 00 \\ 2: 1 E-01 & 45 E & 00\end{array}$ I. $3 E-0$ ?
TESTES 1. BE- OS 4. $:$ SE-09 3: 9 OE- OS 1.9E-05 I: $Y$ EE 02 5. $7 E-02$ : $3 E-03$ $1.6 E-02$ 5: $3 E-03$ I. $3 E-03$ $1.6 E-02$ i. $1 E-04$ $2.1 E-05$
$5.00-02$ 1. SE-02 $2: 9 E-05$
$1.2 E-05$ 9. SE- 06 5.:1E-05 $2.8 E-08$
$1.6 E-06$ I.2E 1:2E 1:2E-OO $1.2 E$ OO $9.0 E-04$
$1: 2 E-0.4$ $1.1 E 00$ $1.1 E=06$ 2. IE-OI
THYMUS $1.8 E-03$
$6.6 E-04$

1. $7: 4 E-09$

2:AE-04 $2.4 E-O A$
$6.7 E-02$ $2.3 E-02$ 5 $1: 7 E-02$
$1: 3 E-03$ $1.6 E-02$
$1.3 E-03$
$1.3 E$ 5 . $3 E-02$

7:2E-03

$1.6 E-02$

$1.2 E-03$
1
2

2. OE-OA

$1.5 E-02$
$2.1 E-03$

$1.0 E-04$

6.:AE-05

3. $O E=04$

1:1E-04

政

3: TE-O

3. $0 E-03$
$3: 6 E-0.04$

3. $7 \in=0 J$

3: $3 E=-0.3$

9.: YE-OY

$1.1 E-06$

3.:6E-03

$4.6 E-06$
$3.2 E-06$

$4.2 E=06$
$9.9 E=03$
$1.9 E=02$

$\begin{array}{r}1.9 E-02 \\ \hdashline 3 E=03\end{array}$

$5.9 E-04$
$1.4 E-05$

$6.4 E-03$

$5: 7 E-03$

$4.2 E=03$
$5.0 E=03$

$4.7 E-03$

$7.3 E-03$
$2.8 E-02$

$1.2 E=01$
$3.3 E=01$

THYAOIO UTERUS

The-03 $2.0 E-04$ z:2E-0. SO $2.5 E-00 \quad 1.1 E-00$ TE-OS 1.2E-OA -6E-0S 1.JE=04 $2.3 E-02$ 2.3E-02 $0.2 E-03$
1. I.TE-02 I:TE-O2 $1.3 E-03$
5 $106 E-02 \quad 1.6 E-02$ $5.4 E-02 \quad 5 . A E=02$ $1.6 E-02 \quad 1.6 E-02$ $5.3 E-02 \quad 5.3 E \rightarrow 02$ I.2E-03 1.BE-O2 $1: 1 E-04$
$5: A E-05$

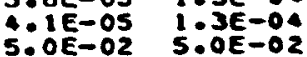
$1.5 E-02 \quad 1 \cdot 5 E-02$ 9.3E-05 $1: 1 E-04$ $2.1 E-05 \quad 5: 1 E-05$ $1.1 E-042.3 E-04$ $1.3 E-07$
$1.5 E-07$ $1.1 E-03$ S. $3.5 E-04$ 3.SE-0. (3) 3. $7 E-03$ 3. 3 E-0. B.:BE-07 $3.2 E-07$ 
INHALAT TON DOSES ÂT I VEAR (REMTMICROCURIE)

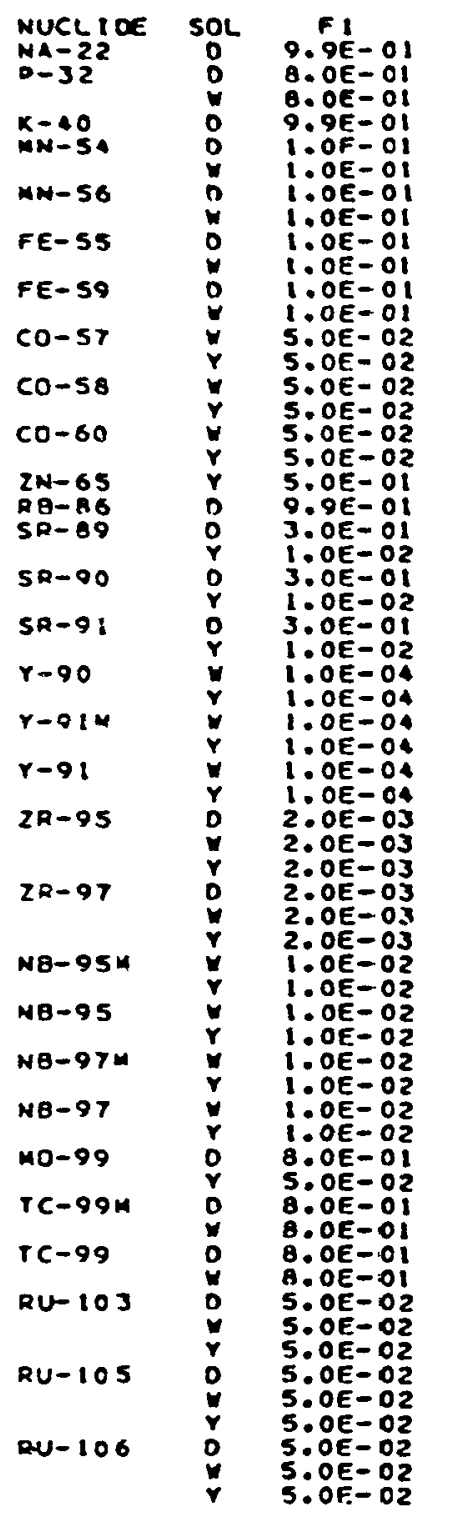

\begin{tabular}{|c|c|c|}
\hline 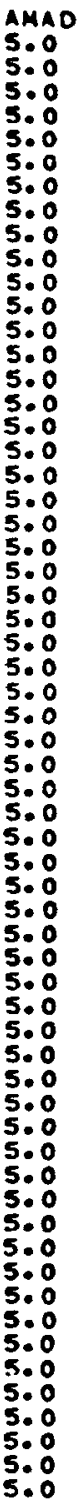 & 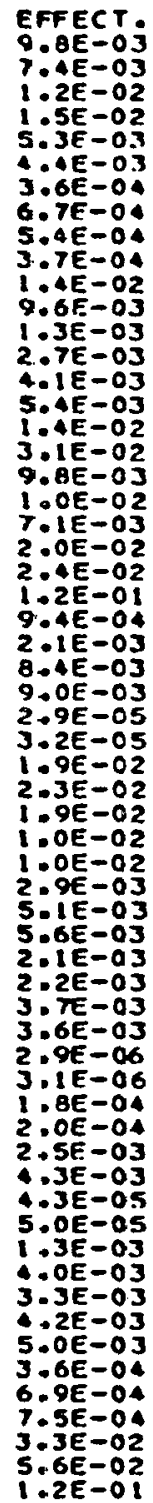 & 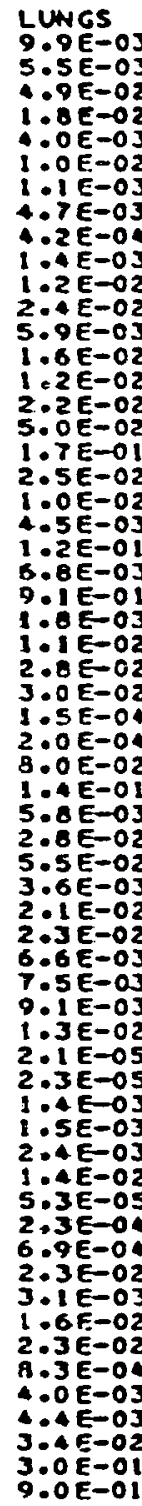 \\
\hline
\end{tabular}

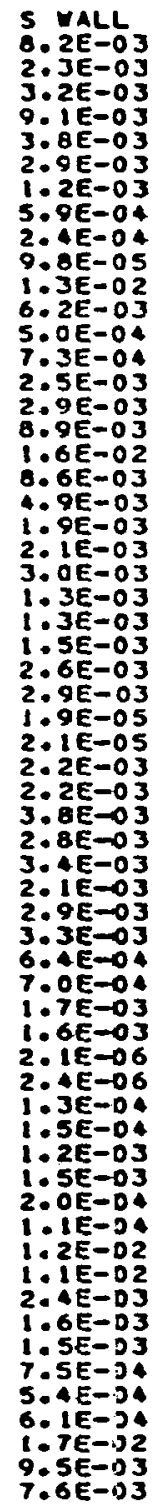

\begin{tabular}{|c|c|c|c|}
\hline & 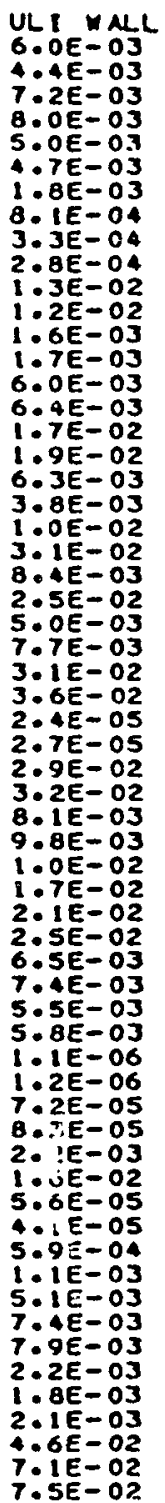 & $\begin{array}{l}\text { LLI } 7.04 \\
1.0 E-03 \\
1.0 E-02 \\
1.9 E-02 \\
8.4 E-03 \\
5.5 E-03 \\
6.0 E-03 \\
7.2 E-04 \\
3.2 E-04 \\
5.2 E-04 \\
6.6 E-04 \\
1.9 E-02 \\
2.4 E-02 \\
3.6 E-03 \\
3.9 E-03 \\
1.1 E-02 \\
1.2 E-02 \\
3.2 E-02 \\
3.3 E-02 \\
8.0 E-03 \\
1.1 E-03 \\
2.9 E-02 \\
9.0 E-02 \\
2.8 E-02 \\
9.7 E-02 \\
5.4 E-03 \\
8.4 E-03 \\
7.5 E-02 \\
8.6 E-02 \\
5.2 E-05 \\
5.8 E-05 \\
8.4 E-02 \\
9.4 E-02 \\
1.6 E-02 \\
2.3 E-02 \\
2.5 E-02 \\
2.5 E-02 \\
3.1 E-02 \\
3.6 E-02 \\
1.6 E-02 \\
1.8 E-02 \\
1.1 E-02 \\
1.2 E-02 \\
2.5 E-07 \\
2.6 E-07 \\
1.7 E-05 \\
1.8 E-05 \\
1.5 E-03 \\
3.7 E-02 \\
3.8 E-05 \\
2.7 E-05 \\
1.5 E-03 \\
3.1 E-03 \\
1.1 E-02 \\
1.8 E-02 \\
2.0 E-02 \\
1.9 E-03 \\
1.9 E-03 \\
2.2 E-03 \\
1.1 E-01 \\
2.0 E-01 \\
2.2 E-01\end{array}$ & 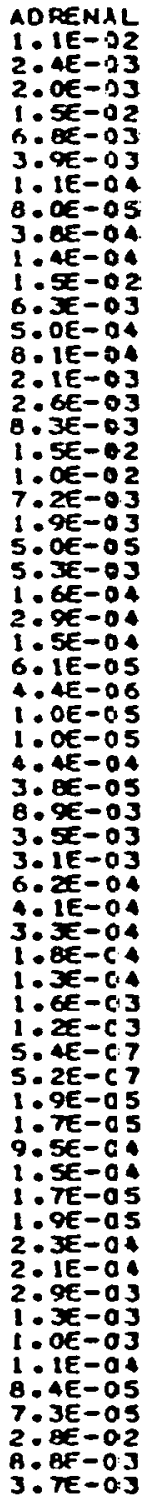 \\
\hline
\end{tabular}

BL MALL 2. 2 . -03 2. $O E-03$ $1.5 E-02$
$2.8 E-03$ 1. $6 E-03$ $3.4 E-05$ 3. $\mathrm{BE}-0.4$

$1.4 E-04$
$1.3 E-02$

$1.3 E-02$
$5.2 E-03$ $3.6 E=0$ $1.6 \mathrm{E}-03$
$\mathrm{~A}-03$

$1.2 E-03$
$5.6 E-03$

4. IE- 03

C. BE-03

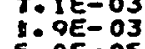

$5.3 E-03$

1. $6 E-04$
$3.1 E-04$

$1.12 E=04$
C. $2 E=05$

i. $9 E-06$

S. $8 E-07$

4. $3 E=04$

3. $A E-05$

$1.5 E-03$
$9.2 E-04$

7. $1 E=04$

3. $7 E-04$

B. SE-05

B:1E-03

9. OE- DQ

4. $3 E-08$

2. $4 E=06$ T. $2 E-04$

7. OE-06

2. $3 E-04$

3. $0 E-03$ 1: $2 E-03$

$\therefore: 2 E-04$

3. 5E-05 a. $6 E-03$
$2.9 E-03$

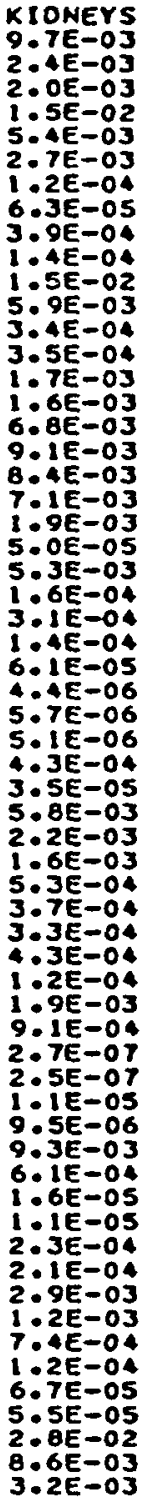

CTVER
CI.6E-OJ

$1.0 E-O J$
. OE-OJ

$1.5 E-02$

$1.7 E-02$
$7: T E-03$
2

$2.1 E=0$

$1.2 E-03$

$4.5 E-0$.

$1.0 E-O 2$

A: $4 E-0$.

$2.9 E-03$

$2: 2 E-02$

$1.8 E-02$

$8.8 E-03$
$7.1 E-03$

$: 1 E-03$
$1: 0 E-03$

5:0E-05

$1.6 E-04$

2:1E-DA

$1.6 E-03$

$2.2 E-05$

$101 E-05$

$1: 5 E-03$

$2.4 E-03$

$6: 0 E-04$

$4.5 E-04$

3. $9 E-0 A$
1. $5 E=0 A$

$1: 3 E-04$
$1.3 E=03$

$1: 2 E-03$

S.AE-07
$5.1 E-07$

$1: 9 E-05$

$1: 7 E-05$

$6.6 E-04$

$1: a E-05$

$2: B E-04$

$1: 3 E-03$

i:1E-0

8.0E-05

$2.8 E-02 \quad 2.8 E-02$ $\begin{array}{ll}8: 7 E-03 & 8: 6 E=03 \\ 3.7 E-03 & 3.3 E-03\end{array}$

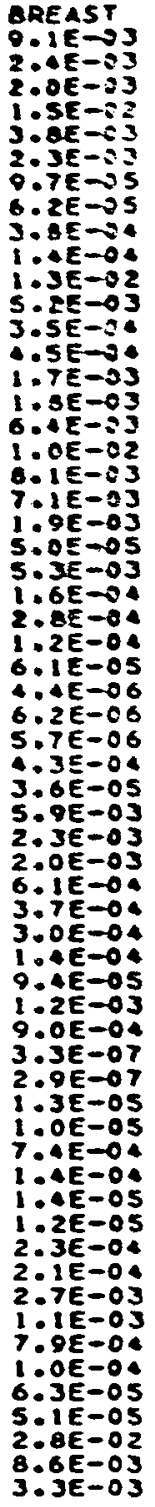


I MHalat ton doses at I vear

(REM/NICROCURIE)

\begin{tabular}{|c|c|c|}
\hline $\begin{array}{l}\text { NUCL } D E E \\
N A=22 \\
P-32\end{array}$ & $\begin{array}{c}\text { SOL }_{0} \\
0 \\
0\end{array}$ & $\begin{array}{c}F 1 \\
9.9 E-01 \\
8.0 E-01\end{array}$ \\
\hline $\begin{array}{l}K-10 \\
M N-5 .\end{array}$ & D & $\begin{array}{l}\text { OOE O OI } \\
1.0 E=01\end{array}$ \\
\hline$M N-56$ & $n$ & I.OE-OI \\
\hline$F E-55$ & D & $1.0 E=01$ \\
\hline$F E-50$ & 0 & $\begin{array}{l}1.0 E-O 1 \\
\text { OOE O }\end{array}$ \\
\hline$c 0-5\rangle$ & 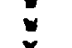 & $5.0 E-02$ \\
\hline co-se & $\frac{\pi}{r}$ & $5.0 E-02$ \\
\hline$C O-60$ & 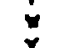 & $O E-02$ \\
\hline $\begin{array}{l}2 N-65 \\
R B-B 6 \\
S R-89\end{array}$ & $\begin{array}{l}r \\
0 \\
0\end{array}$ & $\begin{array}{l}5.0 E-01 \\
9.9 E-O 1 \\
3.0 E=01 \\
.0 E-01\end{array}$ \\
\hline SR-90 & 0 & 3. OE 01 \\
\hline $5 R-91$ & D & $\begin{array}{l}0 E-02 \\
0 E-01\end{array}$ \\
\hline$r-90$ & $y$ & OE- \\
\hline$r-91 \mu$ & 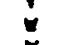 & $O E=0$. \\
\hline$r-91$ & $\checkmark$ & $O E-O A$ \\
\hline $2 A-95$ & & $\begin{array}{l}2.0 E=03 \\
2.0 E-03 \\
2.0 E-03\end{array}$ \\
\hline $2 R-97$ & & $\begin{array}{l}2.0 E=03 \\
2.0 E=03 \\
2.0 E=03\end{array}$ \\
\hline NB-95M & $y$ & $\begin{array}{l}: 0 E-02 \\
: O E=02\end{array}$ \\
\hline NB-OS & $y$ & $\begin{array}{l}1.0 E-02 \\
.0 E=02\end{array}$ \\
\hline NB-OTM & $y$ & $1.0 E-02$ \\
\hline$N B-97$ & $r$ & $\begin{array}{l}O E-02 \\
O E=02\end{array}$ \\
\hline MO-99 & 0 & $8.0 E-01$ \\
\hline rC-99M & o & B. OE $=01$ \\
\hline$T C-99$ & 0 & B.OE-0I \\
\hline$R U-103$ & & $\begin{array}{l}5.0 \varepsilon-02 \\
5.0 \varepsilon-02 \\
5.0 \varepsilon-02\end{array}$ \\
\hline$R U-105$ & & $\begin{array}{l}5.0 E-02 \\
5.0 E=02 \\
5.0 E-02\end{array}$ \\
\hline$R U-106$ & $\begin{array}{l}0 \\
y \\
y \\
y\end{array}$ & $\begin{array}{l}5.0 E=02 \\
5.0 E=02 \\
5.0 E-02\end{array}$ \\
\hline
\end{tabular}

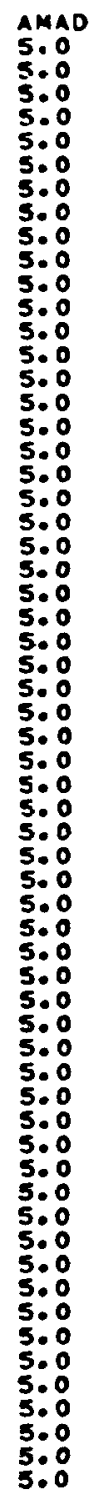

OVARIES PANCREAS GRAIN R RAR ENDJST

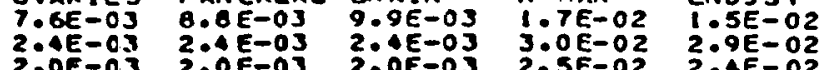
2.:0e-0.3

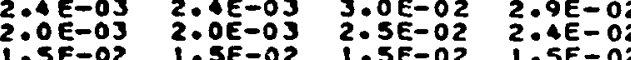

SKIN

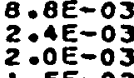

SPLEEN SPLEEN TESTES

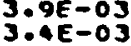
s.0. 3 $6.36=03$
3 $3: 11 E-03$ $1.7 E-04$ $1.5 \mathrm{E}-04$

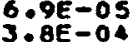
1.7e-05 $3.06-04$
$1.996-04$ 2.6E-05 7 JE-05 $3.1 E-03$ $2: 0 E-03$ a THYMUS THYROTD UTERUS $1.1 E-021 . A E-04$ $3.96-043.7 E=04$ $5: 2 E=05$ $1: 6 \mathrm{E}-03$ 3 :SE-03 2:

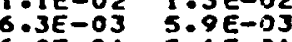
$6.00-0.4$
5

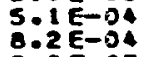
$3 \mathrm{BE}-03$ (1) - $8.5 E-03$

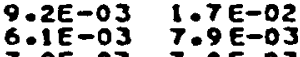
$3: 06-03$ $7: 0 E-03$
$: 00=03$ i $1 E-02$

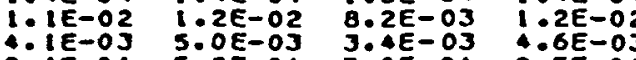
$40.06=04$

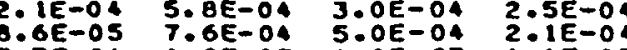

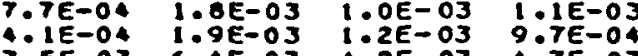
6.4E-03 $4.2 E-03$ $\begin{array}{lll}2: 7 E-03 & 1.0 E-02 & 7: 1 E-03 \\ 7.1 E-03 & 9.0 E-03 & 5.6 E-03 \\ 1 & 0 E-03 & 3\end{array}$ PIE-03 2.8E-02 2.SE-02 $5.0 E-05 \quad 5.0 E-05$ 5.:BE-O4 $\begin{array}{ll}2.1 E-02 & 3.2 E-02 \\ 5.7 E-04 & 8.4 E-04\end{array}$ S.:TE-OA $3.3 E-03$ 5. $5 E-0$ $\begin{array}{lll}1.6 E-04 & 3: 3 E-03 & 5.0 E=03 \\ 1.6 E-04 & 7: 6 E-04 & 6: 7 E=04 \\ 1.6 E-05 & 1.6 E-0.04 & 8.9 E-05\end{array}$ 作 $\begin{array}{rl}\because 1 E-04 \\ 6.1 E-05 & 2.2 E-04 \\ 3 & 0.1 E=05\end{array}$ $1.6 E-04$ $6.1 E-05 \quad 1: 6 E-03$ 1:6E-03

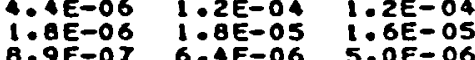
$2 \cdot 1 E-02$ 2.1E-02 $3.0 \mathrm{c}$ $A \cdot A E-06$ $1: 3 E-05$

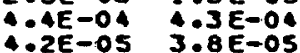
$6.4 E-03 \quad 5.1 E-03$ $\begin{array}{ll}3.6 E-03 & 2.7 E-03 \\ 2.9 E-03 & 3.3 E-03\end{array}$

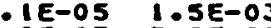
2: $2 E=03$ $3.8 E-02 \quad 3.1 E-0$ $1: 0 \mathrm{E}-02$
$2.7 \mathrm{E}-03$ $7.1 E-01$
$7.3 E-02$ $1.3 E-03$ $6.3 \mathrm{E}-04$ S:AE-DA $2.7 E-03$
$2.5 E-03$ $2.4 E-03$

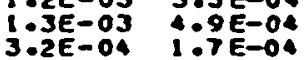
$.4 E-048.8 E-04$ 3: $1 E-05$ 1.2E-OA $2: 2 E=04$ 2.1E-OS 2.0E-04 2:TE-OA $7 \cdot 9 E-04$ $2.6 \mathrm{E}-03$ 2. TE-03 I:S E-03 $2.6 E-03 \quad 1.4 E-03$ 1.1E-0 $6.1 E-07$ $9.7 E-08$ 3.5E-OT $1.1 E-02$
$1.9 E-03$
$2.7 E-07$

$\begin{array}{ll}2.2 E-07 & 1.5 E-07 \\ 1.1 E-05 & 8.2 E-06\end{array}$ (a) $5.9 E-03$
$7: 1 E-03$
$1.02 E-03$ $7: 1 E-03$
$1: 9 E-03$

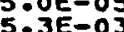
$1.6 E-04$ S.8E-OS $6.11 E=05$
$4.4 E-06$ $3.6 E-06$ $4.3 E-04$ $3.3 E-05$
$4.8 E-03$ $4.8 E-03$
$1.6 E-03$ $2.2 E-04$ $1 \cdot 3 E=04$ $4.6 E-05$ $4: 5 E-04$ $1.2 E-0$ $9.4 E-05$ $2.0 E=03$
$7: 3 E=04$ $7.3 E-05$ 2.1E-05 $1 \cdot A E=04$ 1:1E-02 4:0E-03 $1.0 E-04$
$7.0 E-04$ $2: 0 E-03$ 8. 3E-O4 $20.9 E-04$ $1.4 E-02 \quad 1: 8 E-03$ 7 IE-OJ $7.0 E-03$ $1.9 E-03$ i. $9 \varepsilon-03$ $5.3 E-03$ 5.0E-0S 1.6E-O4 $1.6 E-O$ $1.7 E=04 \quad 3.6 E-05$ 1.

$100 E-05$
$100 E-05$

$3: 3 E-04$ A. $3 E-04$

$4.9 E-033.6 E-03$

$2: 5 E-03 \quad 1.1 E-03$

TRTE-OA $1: 7 E=0$

$3.0 E-04 \quad 1.1 E-04$

1.6E-04 6.5E-05

$2.1 E-03 \quad 1.2 E-03$

$1.1 E-06$
0.06

5. $9 E-08 \quad 2.8 E=07$

: $7 E=08$

. $0 E=07 \quad 1.3 E-006$ 1. BE-OS 6.6E-O $7.6 E-04$
$1.6 E-04$
$1.6 E-0 E-05$ $7.1 E-04$
$3.0 E-03$ $6.4 E-04 \quad 1.9 E-04$ I.: $1 E=05$ $2.8 E-05$
$2.6 E-05$ $\because 3 E-05$
$i: 3 E-05$ $3.0 \varepsilon=0.04$ $3.0 \mathrm{C} \cdot 03$ $2.4 E-04$
$8.9 E-06$
$1.0 E-05$ $2.3 E-O 4$
$2.1 E-04$ $1.7 E-05$

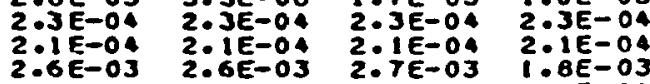
$2: 1 E=03$ $1.8 E-03$
$2.0 E-0.04$ $1: 4 \sum_{-03}$ $7.9 E-041.2 E-03$ $3.0 E-04$
$B .2 E-05$ $1.9 E-04$
$1.4 E-0.04$ $2.7 E-03$ $9.4 E=04$ $1: A E-04$ 2.2E-0S 1.1E-04 $1.2 E=04$ 1:0E-04 A.SE-06 $5.9 E-05$ 3.6E-05 2.5E-05

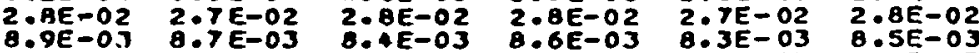
$3.3 E-03 \quad 3.8 E-03$ 
INHALATION DOSES AT I YEAR

(REMAMICROCURIE

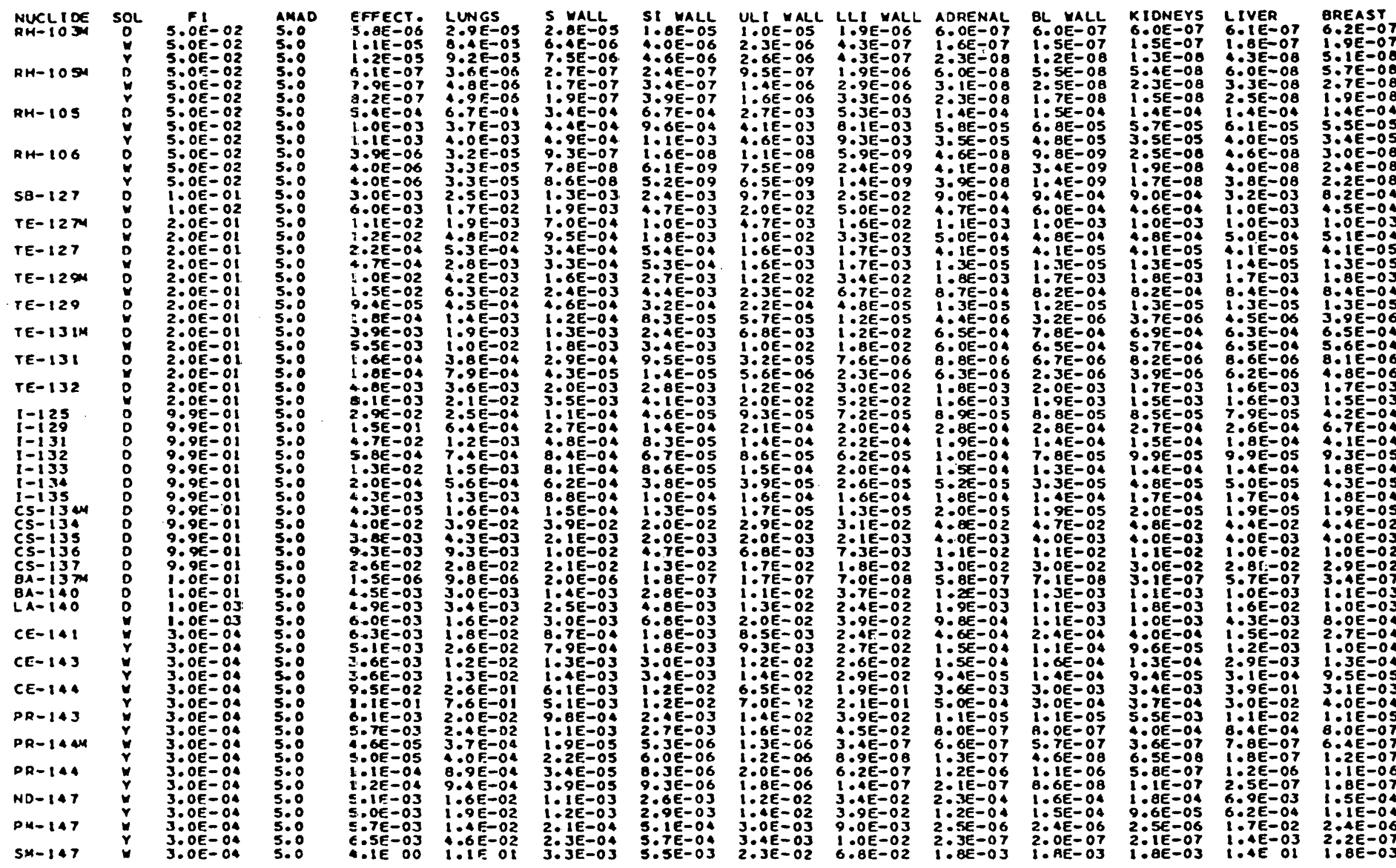


I NHALAT ION DOSES AT I YEAR

(REM/MICROCURIE)

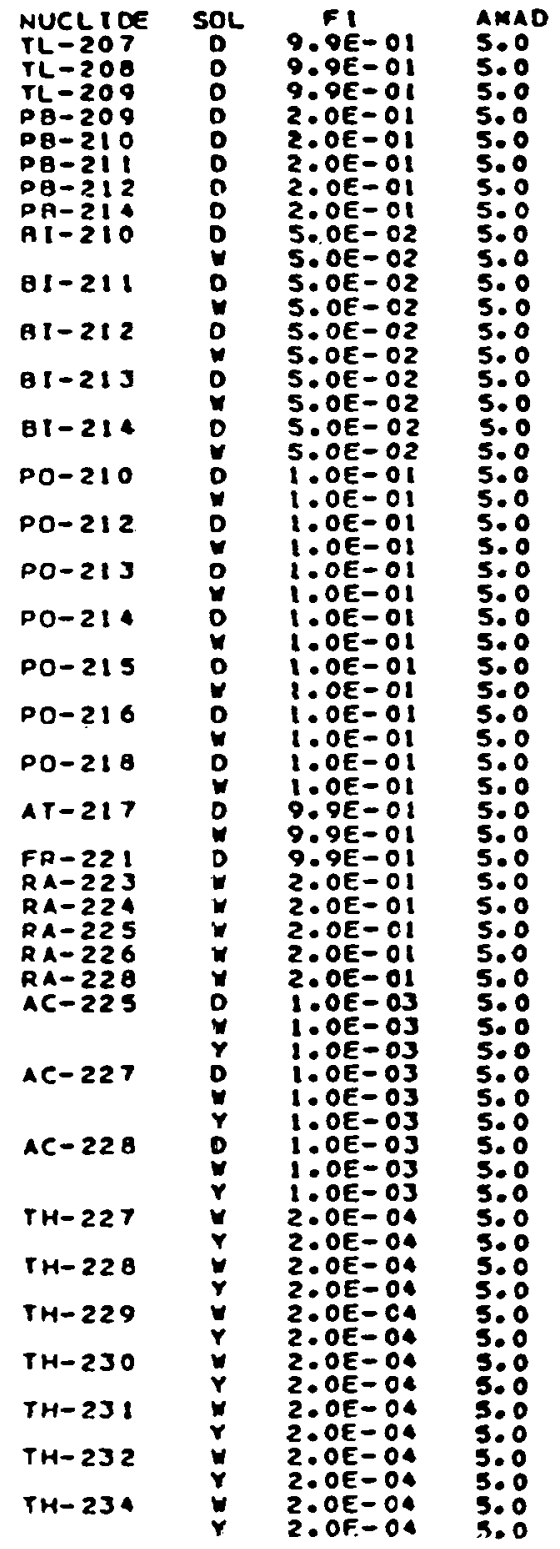

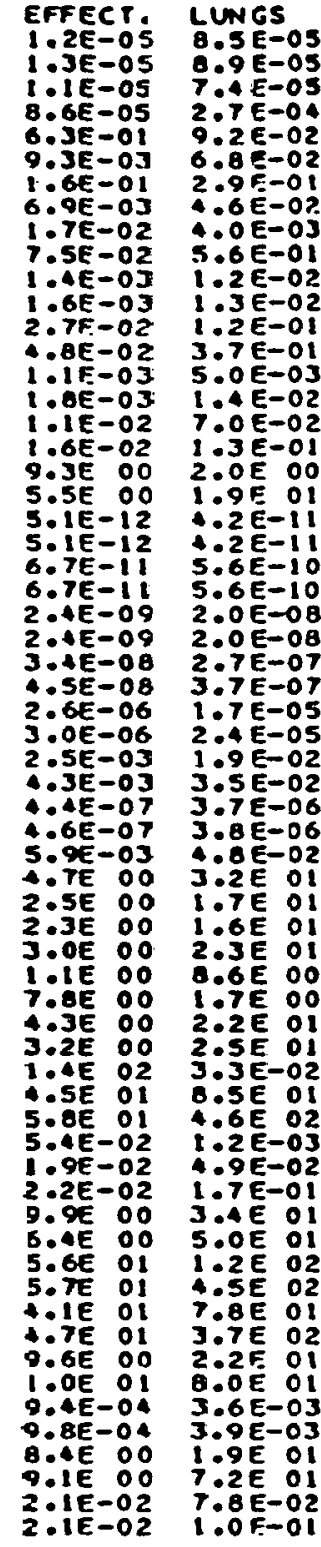

S. YÂLL 1:.e-05 $2.5 E-0$. 2.0 2 $1: 4 \in-03$ $3: 0,0 E-05$

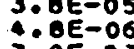
3. 0 EE-0. $7.5 E-0.4$
$3 E-0.4$ :IE-O $1: 1 E-03$ $1: 5 E-00$ $5 . S E-O I$ $2: 4 E-22$ B. $B E-1$ B. $9 E-14$ 3. $6 E-19$ $\because \because 3 E-10$ $1.4 E-07$ 5. P: $3 \in=05$ T) 3: $3 \in=04$ i: $2 E=0$ 1:1

$\rightarrow: 7 \in-02$

3: $2 E-0$

$2.1 \in=02$

s: $1 E=0.03$

3::

Pa:

2. 6 EE-

i: 4 EE

1: 4 E $E-0$

: $: 2 E-01$

2. $6 E-02$

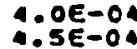
2. $S E-a$ 3:

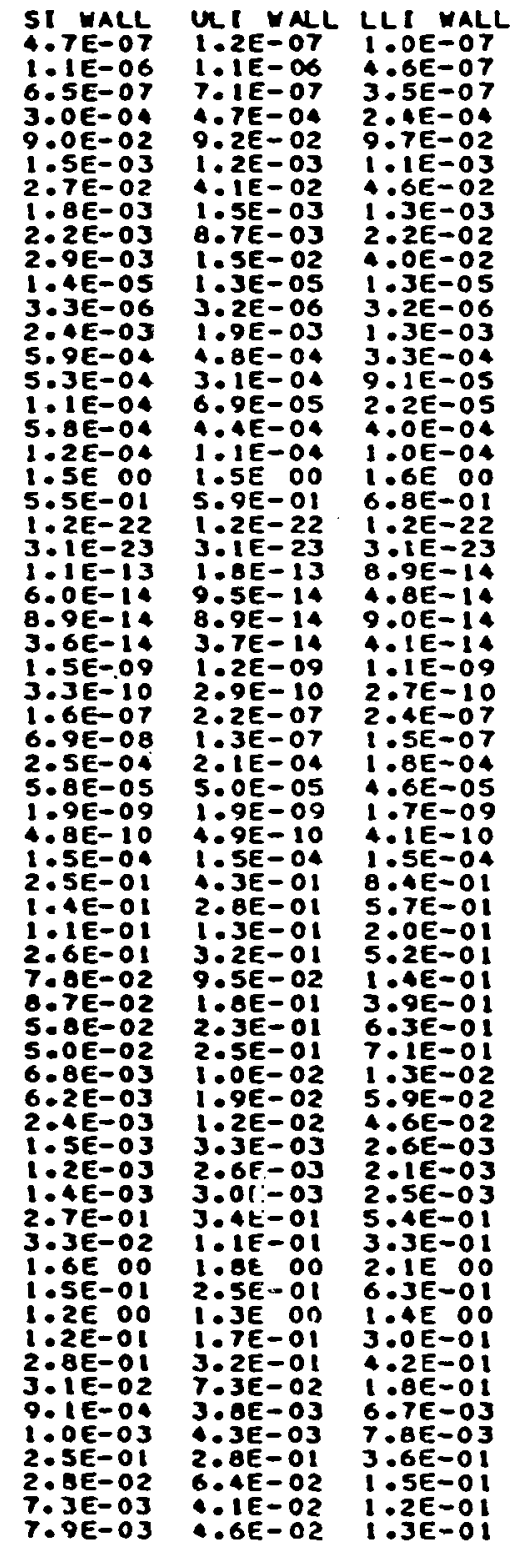

ADRENAL 2. $6 E-06$ 1:TE-OS Q: IE-02 $2.1 E-02$ $1.3 E=03$
$8.1 E=02$ 2.6E-OA $3.3 E-06$ $1 . Z E-0 J$ 3. $3 E-02$ $4.4 E-05$
$41 E-0$. i: IE-0: 1: $1 E-0$ O 1. $2 E-2=$ 3. $1 E-23$ i: $2 E-11$ $\rightarrow$ OEI:TE-OS $2: 7 E-16$
$1: 3 E-07$ $3 . \triangle E-08$ $1: \frac{B E-D}{T E-05}$ 2.OE-D P. $3 E-10$ $2: 1 E=01$ $1 E-0$
$\vdots 1 E-01$ $7.7 E-02$ $7.0 E-02$ $1.7 E-02$ 2.SE 03 $1.6 E-02$
$6.7 E=03$ $3: 2 E=03$
$1.0 E-04$ 1. $2 E=0: 4$ 2.6E-01 $1.9 E-02$ $1.6 E-00$ i. $3 E-00$ $1.1 E-0$
$2.0 E-01$ $2.3 E-02$
$1.6 E-05$ 5: $3 E-0$ 2. $1 E-02$ 5. $3 E=0.04$
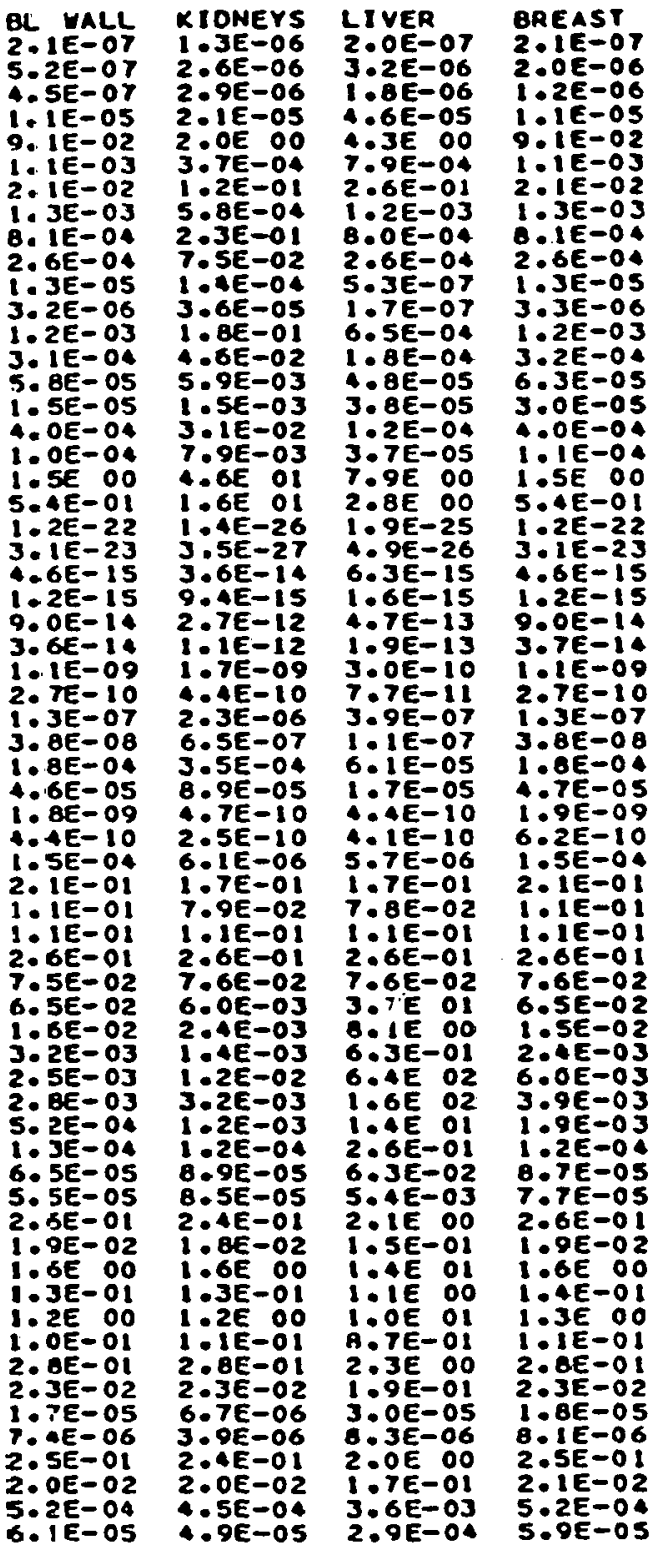
(REM/MICROCURIE)

\begin{tabular}{|c|c|c|}
\hline $\begin{array}{l}\text { NUCLIOC } \\
T L=207 \\
T L=208 \\
Y L-209 \\
0 A-209 \\
0 A-210 \\
P B-211 \\
0 A-212 \\
0 A-211 \\
B 1-210\end{array}$ & $\begin{array}{c}\text { SOL } \\
\text { D } \\
D \\
0 \\
0 \\
D \\
D \\
D \\
D \\
D\end{array}$ & $\begin{array}{l}F 1 \\
9.9 E-01 \\
9.9 E-01 \\
9.9 E-01 \\
2.0 E=01 \\
2.0 E=01 \\
2.0 E-01 \\
2.0 E=01 \\
2.0 F=01 \\
5.0 E-02\end{array}$ \\
\hline$\theta|-2| l$ & $D$ & $5.0 \varepsilon-02$ \\
\hline B $1-212$ & 0 & S.OE- O2 \\
\hline$B:-213$ & 0 & $\begin{array}{l}5.0 E=02 \\
5.0 E-02\end{array}$ \\
\hline$B t-2 t 4$ & 0 & $\begin{array}{l}5.0 E=02 \\
5.0 E=02\end{array}$ \\
\hline$P O-210$ & 0 & 1.OE-OI \\
\hline$P O-212$ & D & I.OE-OI \\
\hline$P O-213$ & $\underline{0}$ & $\begin{array}{l}1 . O E-O 1 \\
1 O E-O 1\end{array}$ \\
\hline$P D-214$ & & $1.0 E-01$ \\
\hline$P O-215$ & & I. OE-OI \\
\hline$P 0-216$ & 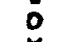 & I.OE-OI \\
\hline$P O-218$ & D & $O E-O i$ \\
\hline$A Y-217$ & D & $9 E-0 I$ \\
\hline $\begin{array}{l}F R-221 \\
R A-223 \\
R A-22 A \\
R A-225 \\
R A-226 \\
R A-22 B \\
A C-22 S\end{array}$ & $\begin{array}{l}0 \\
y \\
y \\
y \\
y \\
0 \\
y\end{array}$ & $\begin{array}{l}9.9 E-O I \\
2 . O E=O 1 \\
2 . O E=O 1 \\
2.0 E=01 \\
2.0 E=01 \\
2 . O E=01 \\
1 . O E=03 \\
1 . O E=O 3 \\
1 . O E-O 3\end{array}$ \\
\hline$A C-227$ & & $\begin{array}{l}1.0 E-03 \\
\text { I. OE }=03 \\
\text { OF }-03\end{array}$ \\
\hline$A C-22 B$ & & $\begin{array}{l}1.0 E-03 \\
1.0 E-03 \\
1.0 E-03\end{array}$ \\
\hline$T H-22 T$ & $y$ & $2.0 E-04$ \\
\hline$T H-228$ & $y$ & $O E-O 4$ \\
\hline$T H-229$ & 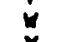 & $O E-04$ \\
\hline$T H-230$ & 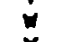 & \\
\hline$T H-238$ & $x$ & \\
\hline$T H-232$ & 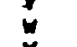 & \\
\hline$T H-234$ & $r$ & $\begin{array}{l}2.0 E-O A \\
2.0 E-O A\end{array}$ \\
\hline
\end{tabular}

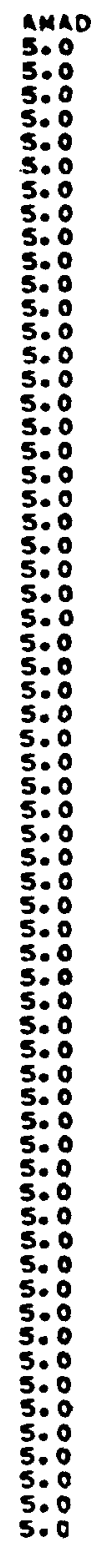

OVARIES PANCREAS BRAIN

MAR

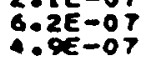

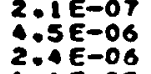

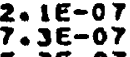
ENDJST i.iE-05

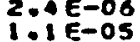
$\because \because 10=02$ i:ie-oj $1.1 E-05$

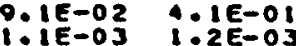

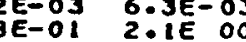
$2.16=02$ $2,1 \in-02$ $2,1 E-02$ $1.3 E-03$ $1.3 E-03$
$B .1 E-04$ $2.6 E=04 \quad 2.6 E-04$ $\begin{array}{ll}3.2 E-06 & 3: 3 E-06 \\ 1.2 E-003 & 1: 3 E-03\end{array}$

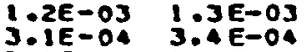
$3: 2 E-05$ $1: 1 E \rightarrow$ $\because .0 E-O 4$ $5: 3 E-0.5$ a:iE $\rightarrow 04$ $2.6 E-04$
$1: 3 E=05$ 3. $2 E-06$ 3. $2 E-03$ $5: 0 E-05$ $\because: O E-04$ 1. $0 E-04$ 1:OE-OA 1:1E-OA S.AE-OI S.AE-OI $1.2 E-22$
$3.1 E-23$ 3:.2E-22 $5.4 E-01$ $1.2 E-22$
$3.1 E-23$ $4.6 E-15$ I.ZE-15 1.2E-15

$9.0 E-149.0 E-14$

$9: 0 E-14$

3.6E-14

2: $7 E-10$

$1 \cdot 1 E-09$
$2,1 E-10$

$1.1 E 09$

: $: 3 E-07$ I:JE-0T $1.3 E-07$
$3 . B E-0 B$ $1: 8 E-04$
$1: 6 E=05$ $1: B E-09$
$4.6 E-10$ $4.6 E-10$
$1.5 E-04$ 2:1E-01 1:1E-01 $7.5 E-02$
$6.5 E-02$ $6.5 E-02$
$1.4 E-023$ 5. $3 E=03$ 3. $5 E-03$ $6 . A E-04$
$8.4 E-05$ $2: 8 E-05$ $2.6 \mathrm{E}-01$ $1: 0 E-02$ 1.:6E :3E DO $1.0 E-01$
$2.0 E-0$ $2: 3 E-02$ $1.2 E-05$
$9.5 E-0 T$ $2.5 E-01$
$2.0 E-02$ S. $1 E-0.4$
$3.8 E-05$ R. MAR 1: $5 E-06$ $3.2 E-06$ $4: 50^{\circ}: 0$

SKIN $1: 2 E-06$ ?:i $1.2 E-03$ $3.9 E-04$ $2.3 E-06$
$1: 3 E-03$ $7.4 E-03$ $2: A E-03$
$5: 0 E-06$ $1: 3 E-06$ $3.5 E-04 \quad 1.6 E-03$ $6.7 E-05$
$3:$ IE-05 $\begin{array}{ll}3.1 E-05 & 5: 0 E-05 \\ 3: 6 E-04 & 1: 1 E-03 \\ 9: 5 E-05 & 2: B E-04\end{array}$ $3.8 E-01$
$3.2 E-23$ $2: 1 E-23$
$: 1 E E-15$ $1.3 E-13$ .TE-10 3.TE-13 $2.0 E-10$
$1: 5 E-07$ A. $1.3 E-04$
$3.4 E-05$ $\begin{array}{ll}5.2 E-10 & 5.2 E-10 \\ 1.0 E-04 & 3.2 E-05\end{array}$ I.AE DO 1.06 OI 6.5E-OI 7.2E 00

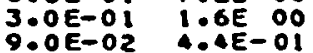
i:IE $2: 4 E$ OO $1: 9 E$ O2 $\because 2 E$ OO i.:3E Oz \begin{tabular}{ll}
$3.0 E$ & 01 \\
$2.3 \varepsilon$ & 00 \\
\hline
\end{tabular} \begin{tabular}{lll}
$2.4 E$ & 03 \\
5 & $9 E$ & 02 \\
\hline
\end{tabular} 5.2E 01 $1: 9 E-02$ Y.TE-03 $8.0 E-01$ 9:9E :O 6.JE OO $\begin{array}{ll}6.1 E & \text { OI } \\ \text { S.OE } & 00\end{array}$ S:OE OO $\begin{array}{llll}9.7 & 02 & 1.0 E & 0 \\ 7.0 E & 01 & 1.3 E-01\end{array}$ 6.2E O1 I:LE-OI :IE-OO $104 E$ OI $22.3 E-02$ $2.5 E-05 \quad 1.1 E-042.1 E-06$

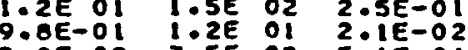
$2: 0 E-O 2$
$1.5 E-03$

$2.1 \varepsilon-02$

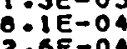

1. $3 E-05$

I. $2 E-03$

S. $5 E-05$

:.OE-:

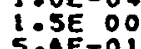

$1.2 E-22$
$3.1 E-23$

$\because: 6 E-15$

9.0E-14

$2: 7 E=10$

3.

$3.6 E-05$
$1.3 E-09$

$5.3 E-10$
$1.5 E-04$

$2: 1 E-01$
$1: 1 E-01$

$2.66=01$

$0.4 E-02$
$1.5 E$

$1.7 E-03$
$3.9 E-03$

$3.2 E-03$

$9: A E-05$

$5.0 E-05$
$3.7 E-05$

$2.6 E-01$

$.9 E-02$ T.SE OO $8.8 E-04$

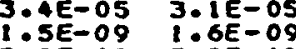

T:ZE OC

$\therefore$ :.SE-O5 $1 . B E-0 S$

A.BE-05 $7.9 \mathrm{E}-06$

$2.0 E-02 \quad 2: 1 E-02$

I.:E-OA $6.8 \mathrm{E}-0.5$
SPLEEN,

$2.0 E-07$
$3: 7 E-06$

$1.8 E-05$

i. $1 E-03$

$2.6 E-042.6 E-04$

$1.6 E-073.2 E-06$

1.2E-03

6.9E-05 5:1E-0S

1.2E-OA T: $3 E=05$

3.TE-05 $1: 0 E-04$

$2.8 E$ OI

S. $A E-01$

. $9 E-26$ 3.1E-23

$1.6 \mathrm{E}-14 \mathrm{i}: \mathrm{.} \mathrm{EE}-15$

$1: 9 E-12$ 3. $6 E-14$

$\begin{array}{ll}3: 0 E-09 & 1: 1 E-09 \\ 3: 6 E-10 & 2.7 E-10 \\ 1 & \end{array}$

$3.9 E-06$

$1.5 E-04 \quad 1.8 E=04$

6. $3 E-10 \quad 1.8 E-09$

7.SE-06 $1.5 E=00$

$1.7 E=012$ 2. $1 E-01$

$1.1 E-01$ 1. 1E-01

(a)

$\begin{aligned} & 2: A E-03 \\ & 1: T E-03\end{aligned} \quad 1: A E-022$

2. $6 E-01$

$1.5 E-03 \quad 1.6 E-03$

1. SE-03 $6.5 E 00$

$2 \cdot x-05$

I: IE-0:

$2.4 E-01$

I: $8 E-02$

1. TE-O!

1: $1 E-01$

$2.3 E-02$

8. $4 E-06$

$2: A E-01$
$2: 0 E-02$

$2 \cdot 0 E-02$
$5.2 E-04$

6. $4 E-05$
5. TE- OI

3. $0 E-05$

2: $6 E-01$

1. $6 E$ OO

1.3E-01

$1.0 E-01$
$2.0 E-01$

$2.3 E-02$

i:SEE-06

$2.5 E-01$
$2.0 E-02$

$4.2 E-05$
THYMUS THYROTO YTERUS

THY $2.8 E-06$ Q:AE-07 5.3E-0?

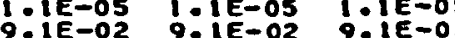
$1.1 E-03$ 1.IE-03 $1.1 E-03$

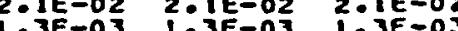
B.IE-04 B.IE-04 B:IE-O4 $2.6 E-04 \quad 2.6 E-04 \quad 2.6 E-04$ $3.3 E-06 \quad 3.3 E-06 \quad 3.2 E-06$ 1.2E-0J (1) $6.3 E-05 \quad 2.4 E-05 \quad 1: 9 E-05$ $1.2 E-04$ 1.OEE-OA $1.0 E-00$ $1.5 E 00$ 1.5E DO $1.5 E 00$ $1: 2 E-22 \quad 1: 2 E-22 \quad 1: 2 E-22$ $3.1 E-23 \quad 3: 1 E-23$ J.1E-23 $4.6 E-15$
$1.2 E-15$ $9.0 E-14$ 9.0E-14 9.0E-14 30.60

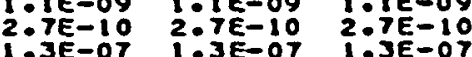

$1.8 E-041.8 E-04$ 1:BE-0.

$4.96-05$ A.TE-05 $4.6 E-05$ 1.

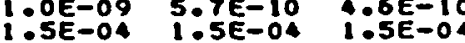
2:1E-OL 2:1E-OL 2:1E-01. 1.1E-01 l. IE-OI 1.1E-O $2.6 E-012.6 E-01$ 2.6E-0I (1) $1.6 E-02$ 1.4E-02 1.6E-02 $4.2 E-03 \quad 3.4 E-03$ 3.1E-03 $3.9 E-03 \quad 3.2 E-03 \quad 3.0 E-03$ I. $1.5 E-045: 9 E-05$ 1:1E-04 $2.6 E-012.6 E-01$ 2.6E-0 $1.9 E-02$ 1.9E-02 $1.9 E-02$ $1.6 E 00$ 1.6E OO 1.6E DO 1.4E-01 $\quad 3$ BE-01 $1.3 E-01$ BE-01 I:IE-OI 1:OE-O $2 . \theta E-01$ 2. $\theta E-01$ 2. $20 \theta-01$

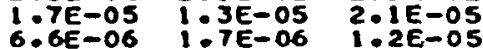
$2.5 E-01 \quad 2.5 E-01 \quad 2.5 E-01$ $\begin{array}{lll}5.3 E-04 & 5: 1 E-04 & 5.3 E-04\end{array}$ 
INHALATION DOSES AT I YEAR (REMPMICROCURIE)

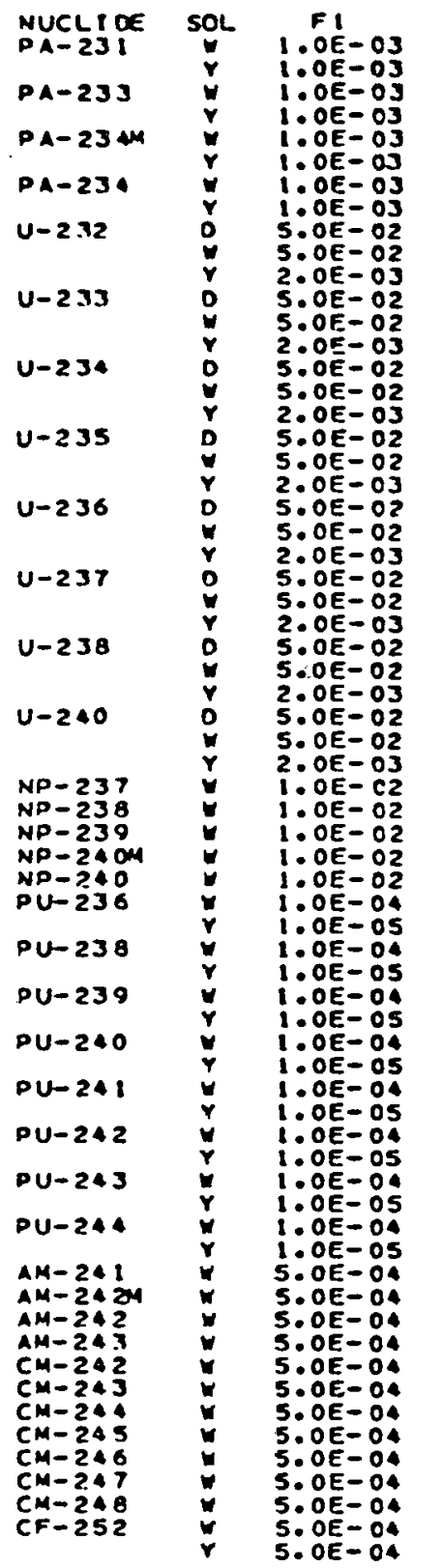

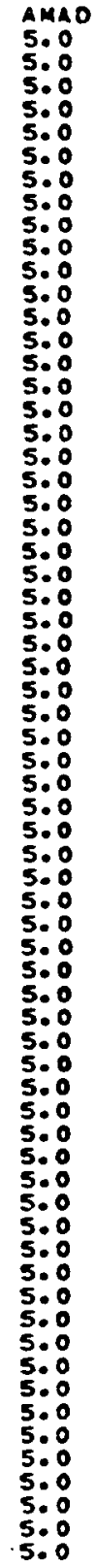

EFFECT: LUNGS

5 YAL

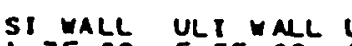

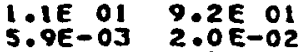

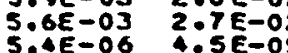

$\begin{array}{lll}5: A G E-06 & 4.5 E-05 \\ 1: 2 E-03 & 0.1 E-03\end{array}$

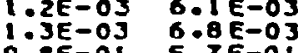

a

2.1E OI $1: 7 \in$ : 02

$\begin{array}{ll}1.0 E & \text { OO } \\ 5.3 E & 01\end{array}$

Y:QE-OI 5.0E-OI

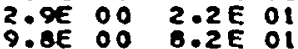

7.AE-OI A.TE-OI

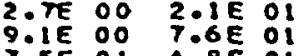

$2.8 E 00 \quad 2.1 E$ OI

$\begin{array}{ll}2: A E-03 & 1: 0 E-03 \\ 3: 1 E-03 & 9.6 E-03\end{array}$

$3.1 E-03 \quad 1: 1 E-02$
$6.6 E-01$
$0.0 E$

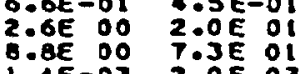

$1.4 E-03 \quad 2.0 E-03$

$\begin{array}{ll}2: A E-03 & 1: 2 E-02 \\ 2: 6 E-03 & 1: 3 E-02\end{array}$

$\begin{array}{ll}9.5 E-00 & 2.2 E \\ 3.5 E-03 & 9.6 E-03\end{array}$

$2.0 E-052.3 E-04$

I.OE O1 $2.6 E$ OI

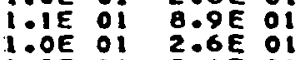

T.2E OI $9.4 E$ OI

9.BE OI B:9E OI

1:LE O1 $8: 9 E$ OI

$9.5 E-03$

$7.1 \mathrm{E}-03$
$\mathrm{E}-02$

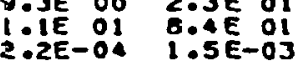

$2.4 E-04 \quad 1.6 E-03$

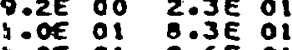

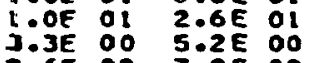

2:Ber

$7.2 \mathrm{E}-02$

P:TE OO 2 :

TiE Oi

2.7E OI

:

$2.3 \mathrm{E}$ OI

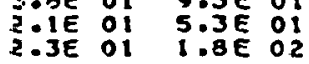

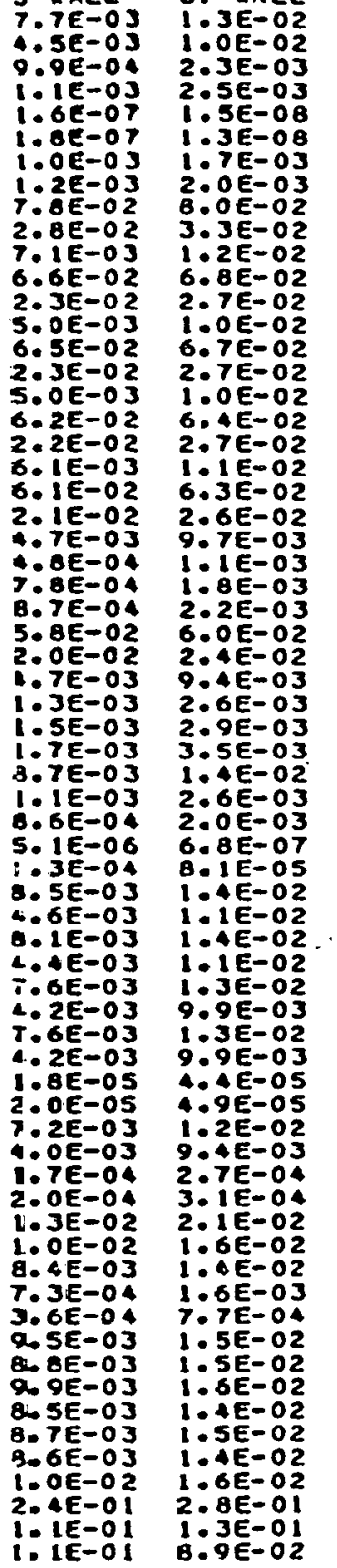

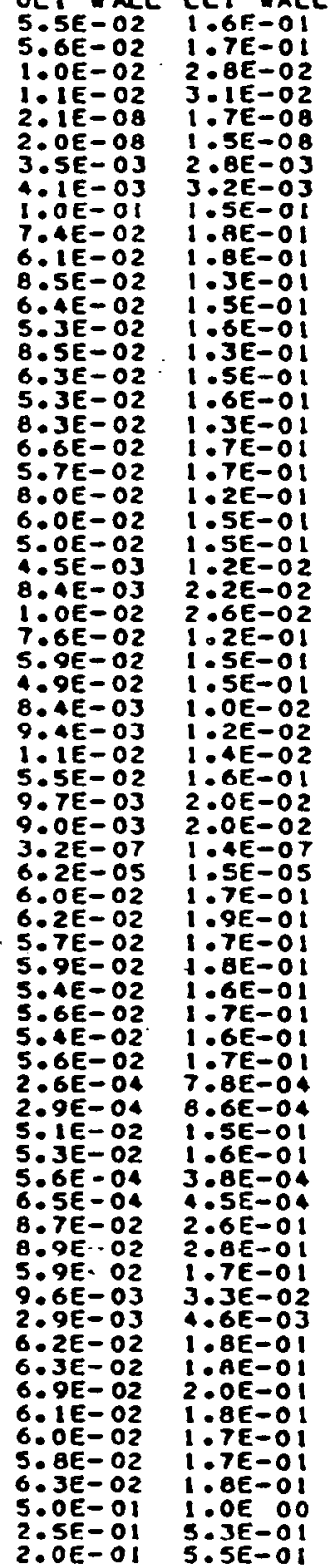

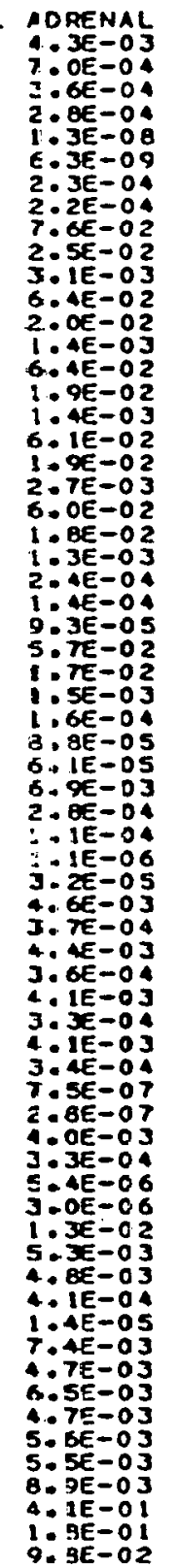

BL

ME-03 RTONEYS LIVER

2. $6 E-04$ 2.2E-O1 $1.5 E-01$

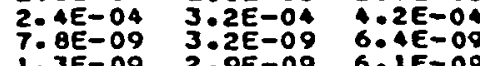

$7.8 E-09$

$1: 3 E=04$

$2: 9 E-09$

$6.4 E=09$

3.1E-0.

DE-02 $1.2 E$ OI $5 . \theta E=02$

2. $0 E-03$

3.:OE-OI

2. $0 E=02$

$3: 8 E: \circ$

$2.0 E-03$

2.2E-01 $2.0 E-02$

$9.7 E 00$ 6.AE-02

1. $9 E-02$

2: $\mid E-01$

$: 9 E-02$
$: A E-03$

$6: 1 E-02$
$1.9 E-02$

2:.9E 00

¿. $5 E-02$

$1.6 E-03$

$1: 0 E-02$

$2.0 E-01$

$1: A E-02$
$2: A E=03$

$2, B E-O O$

0
0

$1.9 E-04$

$1: 0 E-02$

$1.6 E-04$

$2.6 \mathrm{E}-04$

$i: T E=0$

$1.3 E=04$
$1.1 E-04$

作

$\begin{array}{ll}2.6 E-0.1 & 1: 3 E-03 \\ 3.9 E-03 & 6.3 E-05\end{array}$

1. $6 E-04$

1:OE-O3 $7: 1 E-O 5$

$4.4 E-03 \quad 6: 1 E-03$

7:0E-0S

2.7E-04

$1.2 E-04$

$6.9 E-06$

$1: B E-05$

1: $5 E-03$

$3: 7 E-04$

$7.2 E-06$

3:.7E-05

3.JE 01

$4.4 E-03$

3. $5 E-04$

$3.5 E-04$

$3.6 E$ OI

政

$3: J E-04$

3.2E O1

3:2E OI

5. $3 E-07$

3. $3 E-04$

$2.6 E$ O०

$1.5 E-07$

2.1E-0.

3.. $9 E-03$

$3: 9 E-03$
$3.2 E-04$

2.4E 00

1: $\mathrm{BE}=06$

1.

5. $7 E-05$

3.0E :1

1. $2 E-03$

$2.4 E-03$

1.je-0 $2.4 E$. DO

$\begin{array}{lll}3: A E & \text { Ol } & 4: 6 E=03 \\ 1.2 E & 01 & 0.0 E-00\end{array}$

1: $3 E-05$

:TEE-O3

S. TE $\mathrm{TEO}$

$6.6 E-03$
$4.7 E-03$

3.9E-02 $2 . A E-0 S$

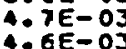

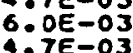

2.3E OI $4.7 E=03$

$5.2 E-03 \quad 3.4 E$ OI $\because: 9 E-03$

\begin{aligned} $06-03 \\$\hdashline $9 E-03\end{aligned}$

$8.5 E-02$

$7.5 E-03$
$3.2 E-01$

3.: $1 E$ :

$5.9 E-03$
$.9 E=03$

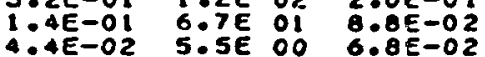




\begin{tabular}{|c|c|c|}
\hline $\begin{array}{l}\text { NUCL IDE } \\
P A-23 !\end{array}$ & SOL & $\begin{array}{c}F 1 \\
1.0 E-03\end{array}$ \\
\hline$P A-233$ & 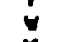 & I.OE-03 \\
\hline$P A-2 J$ AM & 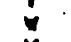 & $1.0 E-03$ \\
\hline$P A-234$ & 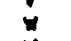 & I.OE-CJ \\
\hline$u-232$ & 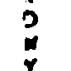 & $\begin{array}{l}5 . O E-O 2 \\
5 . O F=02\end{array}$ \\
\hline$U-233$ & $\ddot{q}$ & $\begin{array}{l}5.0 E-02 \\
5.0 E=02 \\
2.0 E=03\end{array}$ \\
\hline U-234 & $\stackrel{D}{Y}$ & $\begin{array}{l}5.0 \bar{E}-02 \\
5.0 E=02 \\
2.0 E-03\end{array}$ \\
\hline$u-235$ & $\begin{array}{r}0 \\
y\end{array}$ & $\begin{array}{l}5.0 E-02 \\
5.0 E-02 \\
2.0 E=03\end{array}$ \\
\hline v-2 36 & 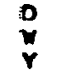 & $\begin{array}{l}5.0 E-02 \\
5.0 E=02 \\
2.0 F=03\end{array}$ \\
\hline$U-237$ & $\begin{array}{l}0 \\
y \\
Y\end{array}$ & $\begin{array}{l}5.0 E-02 \\
5.0 E=02 \\
2.0 E-03\end{array}$ \\
\hline U-238 & $\frac{y}{y}$ & $\begin{array}{l}5.0 \bar{E}-02 \\
5.0 E=02 \\
2.0 E-03\end{array}$ \\
\hline$u-240$ & $\frac{D}{y}$ & $\begin{array}{l}5.0 E-02 \\
5.0 E-02 \\
2.0 E-03\end{array}$ \\
\hline $\begin{array}{l}N P-237 \\
N P=238 \\
N P=239 \\
N P=240 \\
N P=240 \\
P U-236\end{array}$ & 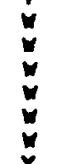 & $\begin{array}{l}1: 0 E-02 \\
1.0 E-02 \\
1: 0 E-02 \\
1.0 E=02 \\
1.0 E-02 \\
1.0 E-04\end{array}$ \\
\hline$P U=238$ & 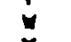 & $O E-04$ \\
\hline$P U-239$ & $*$ & OE-O \\
\hline$P U-240$ & 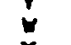 & $1 . O E-0$ \\
\hline$P U-24 I$ & 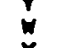 & $O E=04$ \\
\hline$P U-242$ & $y$ & $1.0 \mathrm{OE}-04$ \\
\hline$P U-243$ & $\dot{v}$ & $1.0 E-04$ \\
\hline PU-244 & 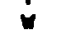 & $D E-O A$ \\
\hline $\begin{array}{l}A M-24 ! \\
A M-242 M \\
A M-242 \\
A M-243 \\
C M-242 \\
C M-243 \\
C M-244 \\
C M-245 \\
C M=246 \\
C M-247 \\
C M-24 B \\
C F-252\end{array}$ & $\begin{array}{l}y \\
y \\
y \\
y \\
y \\
y \\
y \\
y\end{array}$ & 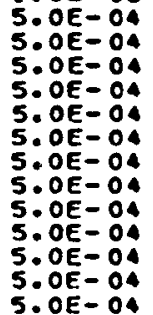 \\
\hline
\end{tabular}

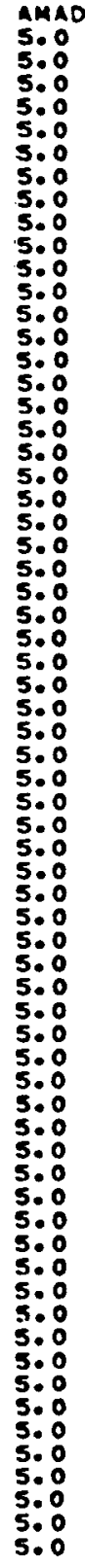

OVARIES PANCAEAS BRAIN

2.SE-04 A.JE-O3 4.2E-O3

$3.0 E=04$
$0.1 E-04$

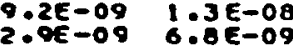

$\therefore .0 E-04$ 3.OE-OA

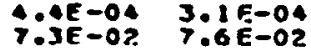

$\begin{array}{lll}2.3 E-02 & 2.5 E \\ 2: 0 E-03 & 3.02 \\ 2 & -0.03\end{array}$

$6.4 E-02$

2.0E-02 $6.4 E-02$

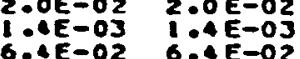

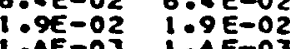

$6.1 E-02$ 6:1E-02

$1.9 E-02$

$\begin{array}{ll}2.2 E-03 & 2.7 E-0 J \\ 6.0 E-002 & 6.0 \mathrm{E}-02 \\ 6 & 0.02\end{array}$

1:BE-02 $1: 8 E-02$

$3.6 E=04$

更

1. $\mathrm{TE}-02$ 1.TE-02

$1.3 E-03 \quad 1.5 E-03$

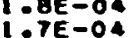

$2.0 E-04$

1.

$\begin{array}{ll}1: 0 E-03 & 3.2 E-04 \\ 0.4 E-04 & 1.3 E-04\end{array}$

$9.5 E-0 B$

$1.0 E-05$

(1)

:OE-OI $3.7 E-04$

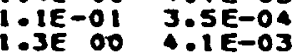

$1.0 E-01$

1.3E 1.001

.5E-05 $2.9 E-07$

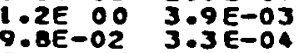

$5.9 E-06$

$5.1 E-06 \quad 4.3 E-06$

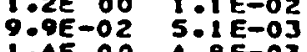

a.QE-01 $3.6 E-04$

$3.2 E-03 \quad 1: 6 E-05$

1.JE

I:AE

6.7 E-03

S.7 E-03

$1: 3 E$ : 00 5.2E-03

S.OF $00 \quad 3.2 E-01$

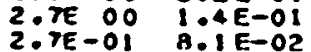

\begin{aligned} $7 E-09 \\$\hdashline\end{aligned}

i: $\begin{array}{ll}2 E-05 \\ 9 E-05\end{array}$

$6.4 E-02$
$1.9 \varepsilon-02$
$0.1 E-0$

$1.4 E-03$

1:9E-02

$1.5 E-04$

$4.8 E-06$

3.6E-05

$4.6 E-03$
$3: 7 E-004$

3. $1 \in=03$

$2.9 E-06$

$8.6 E-0.4$
$: 5 E-03$

$4: Y E-03$
$5: 3 E-03$

$1 . A E-01$
$6.0 E-02$
O.GAR ENDOST

2. $3 E-04$

$7.6 E-02$
$2.4 E-02$

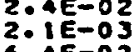

$2: 0 E-02$

$1.01 E-03$
$6.0 E-02$

$1: 8 E-02$
$3 E=03$

T. $7 E-02$

$1: 3 E=03$

$6.3 E-06$
$4.8 E-03$

2. $2 E-05$

$2.7 E-07$
$6.3 E=06$

$4 . A E=03$

3.JE-04

5. 7 E -07

3. $9 E=03$

$2.6 E-07$
$6.4 E-03$

$2: 2 E-04$

5. $0 E-03$

5.: $i E-03$

\begin{tabular}{ll}
$4.7 E-03$ \\
\hdashline $.0 E-03$
\end{tabular}

$6.0 E-02$
$1.0 E-02$

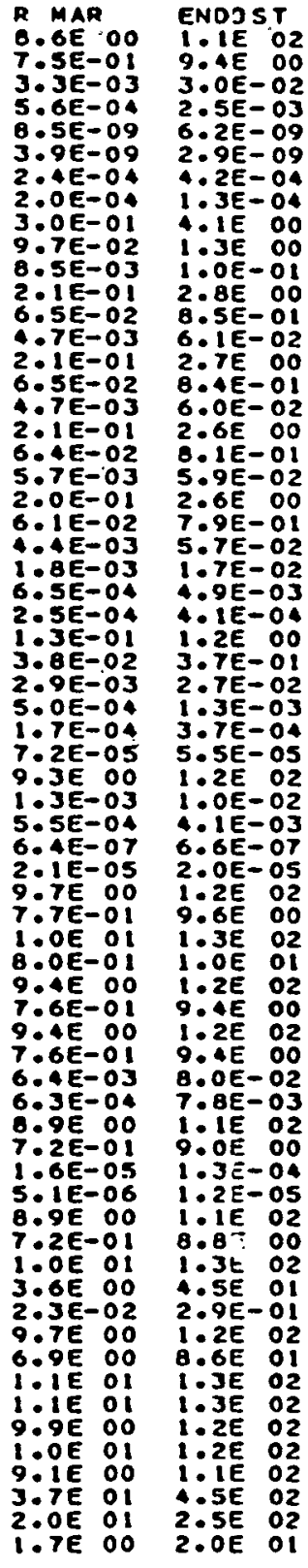

SKIN

$A E-0 \&$
$1.2 E-04$

SE-0: 6.6E-05 2. $9 E-04$ (1)

$9.0 E-05$
$7.5 E-05$

$7.6 E-02$

$2: 4 E-02$

$6.4 E-02$

$1: A E=03$

i: $9 E-02$

$1 . A E-03$
$6.1 E-02$

: $9 E-02$

6:0E-OL

$1 . \Delta E-02$

$1: 3 E-04$

S:Be-05

S: $7 \mathrm{y}^{2} 02$

1: 7 E $3 E-02$

$1.3 E-04$
$5.0 E E-05$
3

$2: 4 E-05$

A. $.1 E-03$

3 . $9 E-05$

i:SE-07

$4.6 E-03$
$3.7 E-04$

$4.4 E-03$

3. $5 E-04$
$4.1 E-0.03$

$3.3 E-04$
$4.1 E-03$

$4: 1 E-03$
$3: 3 E-04$

S.SE-0
$1.3 E E-07$

$3.9 E-03$
$3.2 E-04$

$3.3 E-06$

8.1E-07

$1.8 E-03$
$\therefore 5 E-03$
$3.5 E-0.5$

$2.1 E-04$

$\therefore: A E=03$

$5.1 E-03$

$\therefore .6 E-03$

$\therefore \rightarrow T E-03$
$5: 1 E-03$

5. $2 E-01$

$5.5 E-02$
3.OE-O2

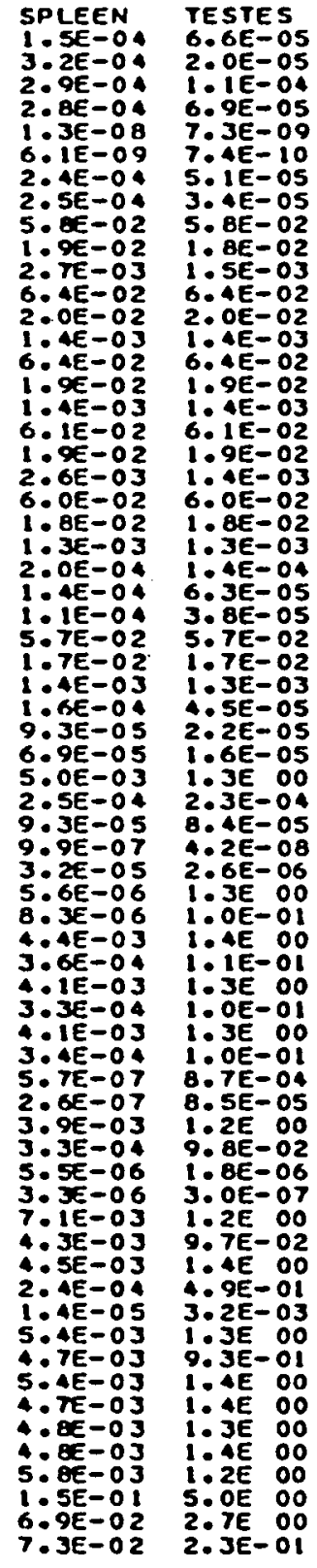

THYMUS

B.7E=04

3.TE -04

I:PE -00

$2.9 E-04$

$7.6 E-02$

$2.5 E-02$
$4.2 E-0 J$

$6.4 E-02$

$1: 4 E-03$

$6: 4 E-02$
$109 E-02$

$1.2 E=3$

$3: 9 E-02$

6.0E-02

1: $\mathrm{AE}-02$

I. $2 E=04$

1.2E-04

5.TE-02

$1.6 E-03$
$1.3 E-04$

9:5E-05

$5: 1 E-03$

$2.5 E-04$

$1.6 E-06$

\begin{tabular}{l}
$4.7 E-05$ \\
\hdashline $.6 E-03$
\end{tabular}

$3.8 E-04$
$4.4 E-03$

$3: 6 E-04$
$4: 1 E-03$

3. $3 E-04$

$4: 1 E-03$
$3: 4 E-04$

5. $7 E-07$

$3: 9 E=03$

6:3E-06

$4.2 E=06$
$7.5 E-03$

$7.1 E-03$
$: .6 E-03$

$2.5 E-04$

$1.0 E-05$
S. $5 E-03$
$4.7 E-03$

$5: 4 E-03$

4 TE -03

$0.0 E-03$
$0.2 E-03$

$1.6 E-01$
$7.2 E-02$
$1.22 E-01$

$1.2 E-02$
$1.2 E-01$
THROI0 UTERUS

BE-04 AjE-04

.4E-05 I:Je-04

-0E-0B A.Ae-0o

. IE-04 2.je-04

.

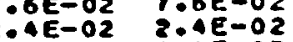

$2.6 E-03 \quad 2: 1 E-03$

$2: 0 E-02$ 2:0E 002

:AE-OJ $1: A E-0 J$

$19 E-02 \quad 1: 9 E-02$

G:AE-02 $6: 1 E-02$

:9E=02 $1.9 E-02$

$6.0 E-02$ 6.0E-02

$\begin{array}{ll}0 E-02 & 1: B E-02 \\ 3 E-03 & 1: 3 E E-03 \\ 3 E E-03 & 2\end{array}$

$\begin{array}{ll}03 E-04 & 2.1 E-04 \\ 505 E-05 & 2.2 E-0.4\end{array}$

.5E-05 $2.2 E-04$

$\begin{array}{ll}1.7 E-02 & 1.7 E-02 \\ 1.7 E-02\end{array}$

$1.3 E-03 \quad 1.3 E-03$

SE-05 $1: 1 E-04$

.6E-03

.0E-04 $41 E-04$

$1.8 E-04$
$3.3 E-05$
$3 E-07$
$2.4 E-07$

. $8 E-05$

$2: 4 E-07$

$4.6 E-0 J$

$\begin{array}{ll}7 E-04 & 3.7 E-04 \\ 3 & \end{array}$

$3.5 E=04 \quad 3: 5 E=04$

$\begin{array}{lll}1 E-03 & 4: 1 E-03 \\ 3.3 E-04 & 3.3 E-04\end{array}$

$\begin{array}{cc}3.3 E-04 & 3.3 E-04 \\ 3 E-003 & 4.1 E-03\end{array}$

$3.3 \mathrm{E}-04$

S.AE-OF

3. $3 E=04$

3. $9 E-03$ Jj: $9 E=03$

$3.2 E-04$
$.2 E-04$

S.2E-06

5. $6 E-03$

$.6 E-03$
$.5 E-03$

. $1 E-06$

$3.8 E-03$
$1.4 E-03$

O $9 E-04 \quad 2.1 E-04$

$1.1 E-05 \quad 1.6 E-05$

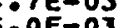

. $7 E-03$

$4: 9 E-03$

$: T E-03$
$3: 1 E=03$

: $7 E-03$

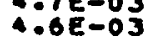

:1E-03

3.8E-02

$5: 2 E-03$
$9: 9 \mathrm{~g}-02$

$3.6 E-02$
$2.3 E-02$ 
INGESTION DOSES AT I YEAR (REM/MICROCURIE)

\begin{tabular}{|c|c|}
\hline $\begin{array}{l}N U C L I \text { OF } \\
H-3 \\
C-14 \\
N A-22 \\
O-32 \\
K=-40 \\
M N-54 \\
Y N-56 \\
F E-55 \\
F F-59 \\
C O-57\end{array}$ & 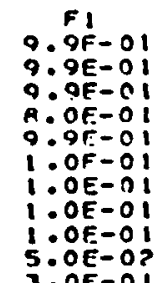 \\
\hline $\operatorname{con}-5 \theta$ & 5.0 \\
\hline$c 0-60$ & $5.0 F-02$ \\
\hline $\begin{array}{l}Z N-65 \\
R A-86 \\
59-89\end{array}$ & $\begin{array}{l}5.0 E-01 \\
9.9 E-01 \\
3.0 E-01 \\
.0 E-02\end{array}$ \\
\hline$S R-90$ & $3.0 E-O I$ \\
\hline $5 a-9 t$ & OE- \\
\hline $\begin{array}{l}Y=90 \\
Y-91 M \\
Y-91 \\
20-95 \\
Z R-97 \\
N B-95 M \\
N R-95 \\
N A-974 \\
N B-97 \\
M O-99\end{array}$ & 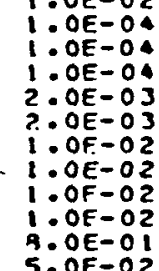 \\
\hline $\begin{array}{l}T C-994 \\
R C-99 \\
R U-103 \\
R U-105 \\
R U-1006 \\
R H-10034 \\
R H-10054 \\
R H-105 \\
R H-106 \\
S B-127\end{array}$ & $\begin{array}{l}\text { B. OE-OI } \\
8.0 E-01 \\
5.0 E-02 \\
5.0 E-02 \\
5.0 F-02 \\
5.0 E-02 \\
5.0 E-02 \\
5.0 E-02 \\
5.0 E-02 \\
1.0 E-01\end{array}$ \\
\hline $\begin{array}{l}T E-12 N \\
T E=127 \\
T E-1294 \\
T E=129 \\
T E-1314 \\
T E=1331 \\
T E-133 \\
1-125 \\
1-129 \\
1-131 \\
1-13 \\
1-133 \\
1-133 \\
1-135 \\
1-135\end{array}$ & 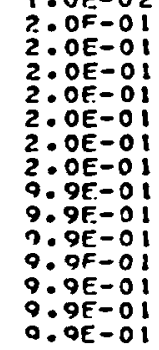 \\
\hline
\end{tabular}

LUNGS

9.

3. $3 E-0 S$

6. $9 \mathrm{E}-05$

2.06-03

(9.

2. AE-OA

1. $2 E-03$

5. $9 \mathrm{E}-03$

5. $2 E-03$

\begin{aligned} 0 \\ \hdashline$E-04\end{aligned}$

.

aic-os

$3.2 E-04$

5. $0 E-0 B$

B.
BE

P.9F-05

6. $5 E-05$

9. $8 E-06$

9. $6 E-05$

T. $3 E-06$

S. $7 E-05$

उE-05

2. $2 F-04$

$2.5 E-04$

2: $3 E=03$

$1 \% 9 E-08$

$1.5 E-05$

2E-OB

$1.6 E-04$
$5.8 E-05$

$3: 4 E-04$
$1: 2 E-05$

S:TE-0.

I. $9 E-O K$

2. 2E-0.

C. AE-D

T. 2E-0:

3. $7 E-0 A$
S. $\theta E-05$
.

$1: a E-04$

$4.6 E-05$
$i .9 E-04$
SI WAL

$9.0 E-0$

5. 0 E -03

?:6F-03

3. $9 \mathrm{E}-03$

8. $6 E-05$

$6.0 \mathrm{E}-03$

7:6E-04

$0.0 E-0.3$
$5 F-03$

$9.9 E-03$

$1: 0 E-02$

$4: 0 E-03$

$A E-03$
$6.1 E-03$

$3.0 E-03$

$2.6 E-03$

$\therefore 9 E-0 J$

6. $5 E-03$
$9.4 E-03$

$1.1 E-04$

: $2 E-02$

3. $4 E-03$

$1.6 \mathrm{E}-05$

$1: 1 E-03$

7.6E-03
3.6E-0.

3. $3 E-04$

$3.0 E-03$
$3,16-03$

5. $9 E-05$

$1.6 E-03$
$5,8 E-07$

5. $5 E-03$

(1)

I:AE- $\triangle A 3$

$5: 1 E-03$

S. $96-03$

5. $9 E-04$

5. $5 E-05$

2:6E-04

$1: 1 E-04$

$1.3 E-04$
$9.0 E-05$
$1.6 E-04$
ULT WALL LLI YALL

IE-OA

: $7 E-03$

SEE -03

$5: 1 E-03$

2. $9 E-04$

$1.3 E-02$

$: T E-03$

$7.1 E-03$

$1.8 E-02$

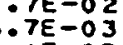

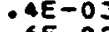

2. $6 E-02$

$0.6 E-02$
$1.0 E-02$

$1.3 E-02$

$\therefore E-02$
$\therefore A E-02$

$1.1 E-0$.

$3.8 E-02$

$4.5 E-02$

.
S. $6 E-03$
$6 E-03$

1. 2E -05

. IE-03

1. $3 E-0$.

$1 . A E-O 3$
$9.2 E-03$

$6: 1 E-03$
$3: T E-02$

政

$3.0 E-0$.
$6.0 E$

(

2.TE-02

$\because \begin{aligned} & 1 E-02 \\ & 3 E-03\end{aligned}$

:OE-O2

$1 . \theta E-02$

2. $9 E-02$

2. $6 E-04$

2. $\triangle E-04$

$2.9 E-04$

Q. $3 E-05$
$3.0 E-04$

\begin{tabular}{|c|c|}
\hline $\begin{array}{l}1 \\
1 \\
A \\
A\end{array}$ & 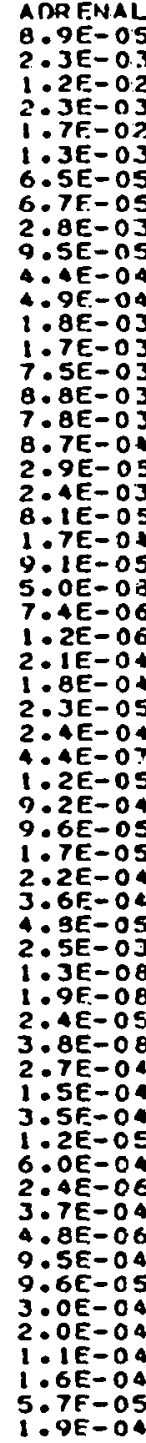 \\
\hline
\end{tabular}

OL WALL

$9.0 \mathrm{E}=04$

9: $8 \mathrm{E}-03$

1. $3 E-03$

$6.7 E-05$

$3.4 E-03$

$2.5 E-04$

$5=115-04$
$1: 3 \equiv-0.3$

$1.28-03$
$3.7 \mathrm{E}-03$
7.06

7. $8 \mathrm{EE}-03$

$7.6 \mathrm{E}-03$
$\mathrm{TE}-03$

8. $7 E-04$

$2.4 E-03$

. IE-05

2. $9 E-04$

2.5E-OA

$8.6 E-06$

$4.4 E-06$

$6.5 E-04$

Э.2E-05

9. $9 E-04$

$2.9 E-07$

$1.6 E-05$
$7.3 E-0.4$

2. AE-0A

2. $2 E-04$

$3 . J E-04$

$1.3 E=04$

$1.4 E-08$

$5.5 E-05$

3 . $3 E-09$

. $E-04$

$3.5 E-04$

. $3 E-04$

$2.5 E-06$

O. $A E-04$

2. $2 E-03$

3. OE-OA

. $6 \mathrm{E}-04$

$9=05$

\begin{tabular}{l}
$1 . S E-04$ \\
\hdashline$. S E-05$
\end{tabular}

: : $9 E-05$
KIONEYS

I: $1 E-03$

?: $3 E-03$

1. $3 E-03$

$6.7 \varepsilon-05$

$3: 1 E=03$
$1: 3 E-04$

$\therefore \quad \Delta E-04$

1: $9 E-0 j$

$3.3 E-03$
$7.0 E-03$

$8.3 E-03$

$8.7 E-04$

$2.9 E-03$

. $1 E-05$

5. 00 E 0.06

S.:OE-OB

.2E-06

$4.1 E-04$

6.
$5 E-O 5$
$5.0 E-04$

$7.6 E-07$

2. SE -05
$9.0 E-03$

2:0E-OS

2.2E-O4

$\because . \theta E-04$

$1: 0 E-04$
$2: 6 E-03$

1. $8 E-08$

3. $9 \mathrm{E}-05$

$4.2 E-04$
3

$3.2 E-04$

1.: $2 E-05$

6. $0 E-04$

$6.1 E-0.04$

$1.3 E-03$

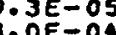

$1.7 E-04$

$1.3 E=04$
$1.6 E-04$

$1.6 E-04$
$6.8 E-05$

6. $8 E-05$
LIVER

$8: 0 E-03$
$0 E-03$

$1,6 E-02 \quad 2.36-0 J$

$0.0 E-0$.

2.IF-OA B.TE-OS

$\because .7 E-03$ 2.OE-OJ

$9.2 E-04$ 3.9E-04

Q.OE-OA $0.6 E-04$

$\begin{array}{ll}2.9 E-03 & 2.0 E-03 \\ 1.3 E-02 & 0.5 E-03\end{array}$

T.7E-0J

$7 . B E-03 \quad 7 . B E-03$

$\begin{array}{ll}8.7 E-04 & 8.7 E-04 \\ 2.9 E-05 & 2.9 E-05\end{array}$

B. IE-OS

$1.7 E-04 \quad 2.0 E-04$

1:AE-OA $1: 3 E-0:$

$9.1 E-06 \quad 6.8 E-06$

2. $\theta E-04 \quad 2.0 E-06$

$\begin{array}{ll}1.9 E-O 4 & 3,0 E-04 \\ 2.9 E-05 & 3,0 E-05\end{array}$

$3.0 F-04 \quad 3.9 E-04$

$1.6 E-05 \quad 1.2 E-05$

$6.5 E-04 \quad 1.3 E-0$.

I.TE-OS I:SE-OS

2. $9 E-04 \quad 2.2 E-04$

$3.2 E-05$
$2.0 E-05$

$1.9 E-O B$

$1.9 E-08 \quad 1.0 E-08$

3.6E-OB 2.5E-OB

$7.4 E-04 \quad 3.7 E-04$

$2.7 E-04$
$3.4 E-04$
$3.5 E-04$

$5 . B E-04 \quad 6.0 E-04$

$2.6 E-06 \quad 2.4 E-06$

$\because 7 E-04$ S. $1 E-0$.

$1.0 E-O 3 \quad 1: 2 E-O 3$
0.03

2.9E-0.

$1.8 F-04 \quad 45 E-04$

$1.0 F-04$ 1.OOE-OA

$\begin{array}{ll}1.5 F-04 & 1: 9 E-04 \\ 5.2 F-05 & 1: 7 E-05\end{array}$

$5.2 F-05$
$1 . T E-04$ 
INGESTION DOSES AT I YEAR

(REM/MICRDCUAIE)

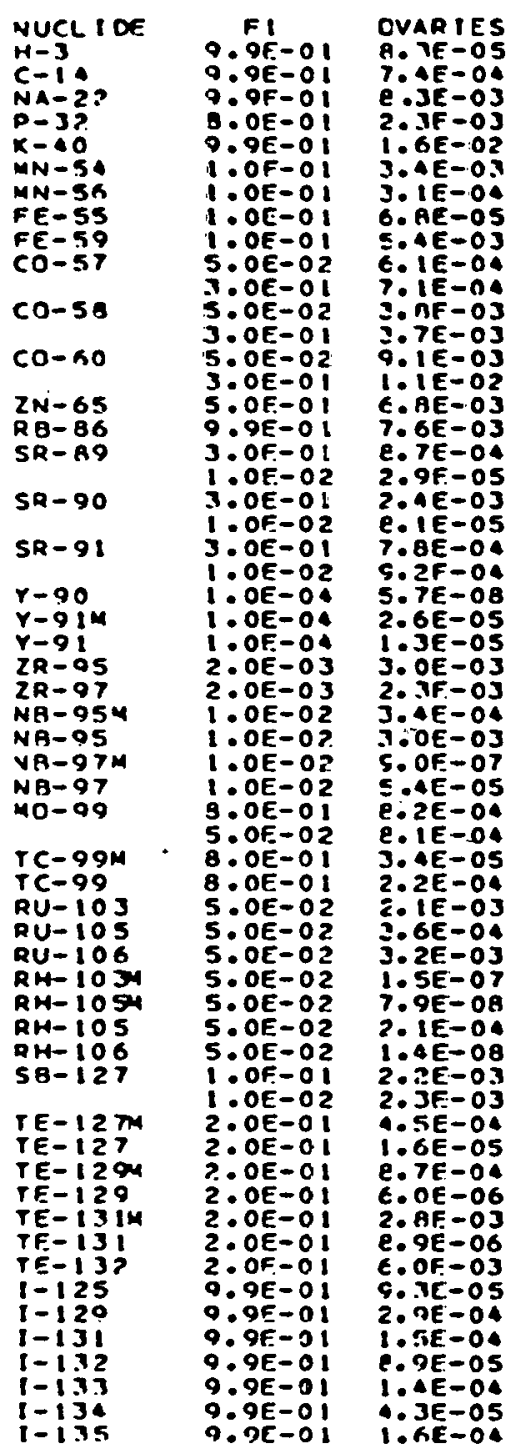

PANCREAS RRAIN

e. IE-OS

OGE-O

० उE-0.3

1.TE-OZ

2. $1 E-0$

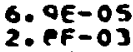

$1, A E-04$

$6.6 E=04$

$1.6 E-03$

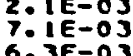

6. 3E-O

B. TE-OA

S.

:1F-OS

AE-D

5. 0 -OB

i. 1 FE-05

2. $0 E-06$

$\because 0 E-04$

$\therefore$ OE-OS

OF-06

6. $1 E-05$

$1 . \triangle E-O A$

3. $2 E-05$

2. 6 E-03

1.6E-OT

!:IE-OP

3. OE-0?

3. IE-DA

3.AE-D4

$1.3 E-05$

5. $9 E-04$

6. $1 E-04$

$1: 1 E-03$

$3.0 E-04$

2. 1 EE-04

2. AE-OA

$2.0 \mathrm{E}-04$
o.jE-OS

: $1 E-02$

1.65 .02

60 of -0.

T:AE $=06$

2. OE -0J

3. TE -04

$2: 1 E=04$

Q $9 \mathrm{E}-0$

5: $3 E=03$

B: $7 E-04$

2. $9 E-05$

8 IF -0 S

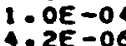

3: 6 E -07

3. 6E -05

5:AE-OS

6. AF -09

2. $1 E=0$ ?

i. 2E -OS

2. $2 E-05$

2. SE -03

4 IE-OO

$1.1 E-05$

i: 盗E-D

$3.0 E-0.05$

1. $2 E-05$

$\because 3 E-06$

$1: B E=04$

O.SE -OA

S. $2 E-04$

S.1E-09

1. $9 E-04$
$3.2 E-05$
$2.0 E-04$

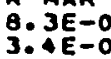

ENOOST
$6.6 E-05$

SKIN

$1.9 E-02$ 1 $6 E-02 \quad 0205-03$

-02 2,BE -02

$1.6 E-02$

$1.8 E-03$

J.

6. $8 E-05$

2. $6 E-04$

政:

?.7E-0 3

6. SE-0J

8.

9. $9 E-03$

5. $3 E-04$

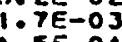

$1.5 E-04$
$2.1 E-04$

萡

i. $9 E-05$

$3 E-04$
$\because: B E=04$

1. $2 E-04$

7. $2 E-04$

$1: 5 E-05$

$3.1 E-04$

2. $3 E-05$

2. $9 E-04$

2. $6 \mathrm{E}-03$

3. $8 E-08$

5. $A E-05$

1. $8 E-08$

$\because 9 E-04$

2. $2 E-02$

1:: $\begin{aligned} & 1 E-02 \\ & \text { SE-06 }\end{aligned}$

B. $8 E-04$

2. $66-06$

$2.1 E-04$

3. $A E-04$

$9.2 E-05$

$1 . T E=04$
$1.1 E=05$
1. TE-OA
. TE $=03$

. $5 E-04$

. $7 E-04$

:2E-03

$4.0 E-03$

促

$4.9 E-04$

$9 E-02$
$2: 6 E-03$

:3E-04

: $6 E-05$

$3.2 E-06$

$1.8 E-05$

$1: 3 E-03$

$14 E-04$

I:OE-0?

: $9 E-06$

$2.4 E-14$

$9.4 E-06$

2. $2 E=04$

. $3 E-05$

$1.9 E-08$

$1.2 E-08$

$1: 1 E=08$

1. $9 E-04$

$\because: B E-02$
$1.7 E-05$

$2 \cdot 5 E-02$

$1: 2 E-03$

3.: $2 E-03$

i. $A E-04$

3. $8 E-04$

$2.9 E-04$
$6.6 E-05$

$1.4 E-04$
$2: 9 E-05$

$2.9 E-05$
$i, A E-04$
8. $3 E-05$

9:SE-O

$1.6 E-0$

: $6 \mathrm{E}-02$

6.7E-OA

6. $9 E-05$

$2.3 E-0.3$

.6E-0:

$1.4 E-03$

$8 E-03$
0.03

: $8 E-03$

更

2. $4 E-03$

: 4 E-O4

$2.6 E-06$

$1.5 E-04$

1.1

$1: 7 E-04$

$4.8 E-06$

.9E-OA

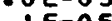

$2 \mathrm{E}-04$

2.5E-05

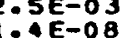

$\because E-09$

$1.9 E=05$

2:1 1 E-0.

1:1E-0

1 . $2 E-05$

$: 7 E-06$

2 SE-OA

B. $2 E-04$

$39 E-04$

3. $3 E-04$

?.

$3 . A E-05$
$1.7 E-O A$

1 उE-OA

$5.6 \mathrm{E}-03$

9.5E-05

6. $0 E-04$

.9E-O

T.: $1 E-03$

B.TE-OA

$2.9 E-05$
$2.4 E-03$

$2 .: 1 E-03$
$8,1 F-05$

$2.3 E-04$

$5.0 E-08$

$2 \cdot 4 E-05$

$1.8 \mathrm{~B}-06$
$3.2 \mathrm{E}-04$
$\mathrm{~S}$

3

$4: 0 E-D 4$

$1.6 E-06$

$3.4 E-04$

$1.1 E-04$

$2.2 E-04$

$\because .2 E-04$

2. $6 E-03$

$\mathrm{S}: 1 E-08$
$3: 3 E-08$

5. $3 E-08$
$3.2 E-05$

1. $6 E-0$ ?

$2.5 E-04$

$3.4 E-04$

5. $2 E-05$

$4.5 E-06$
$5.0 E-06$

I. $3 E-05$

$7: 7 E-0 S$

$2.8 E-04$

$2.06-04$

IOBE-0:

TESTES
$8.3 E=06$
$7.2 E-04$

THYHUS

THYROIO

B. $3 E-05$
$8.9 E-04$

$1: 0 E-02$

2: $7 E-02$

$5.4 E-04$

$1.0 E-05$

$1.6 E-02$
$5 E-04$

. $2 E-05$ 1.7E-05

$\begin{aligned} & 2.6 E-05 \\ & 2.2 E-03\end{aligned}: 9 E-03$

列

3. TE-OA

$1: 3 E=03$

2. $2 E-04$

$1.2 E-03$

5: $9 E-03$

$7.6 E-03$
$.6 E-03$

0
0

$6.4 E-03$

Q. TE-O4

$2.9 E-05 \quad 2.9 E-05$

$\begin{array}{ll}2: 4 E-03 & 2.4 E-03 \\ 8: 1 E-05 & 8: 1 E-05\end{array}$

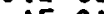

3E-0S

$1: 0 E=04$

5. $0 E=08$

-9E-07

$1: A c=06$

$2.9 E-04$

5. $1 E-05$

5

$2.8 E-06$
$6.7 E-04$

$1: 0 E-04$
$: .2 E-05$

2. $2 E-04$

$2.9 E-05$

1. 3 -OB

1.:OE-0B

$1,1 E-09$

2. 2E-04

3.
$1: 2 E-04$

5. $8 \mathrm{E}-04$

1. SE-06

$1: 5 E-06$

$9.2 E-05$

$2.9 E-04$

I:SE-04

$7.3 E-05$

2. $9 E-O 5$

2. $8 E-05$

6. $7 E-06$

9. $5 E-08$

2.1E-06

$4.4 E-05$

政 
INCESTIOW DOSES AT I YEAR (OEMPHA ACROCURIE)

\begin{tabular}{|c|c|c|}
\hline 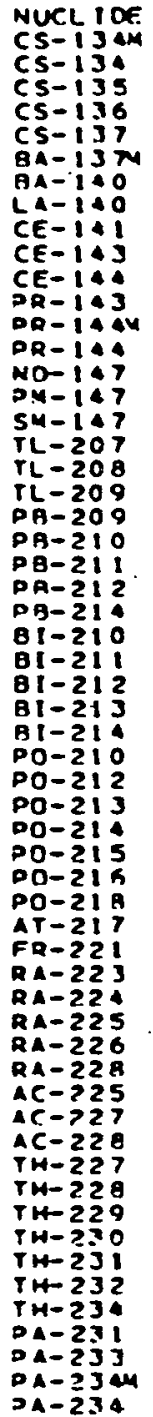 & 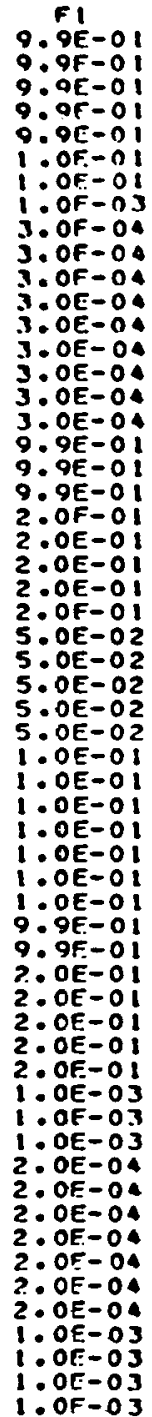 & 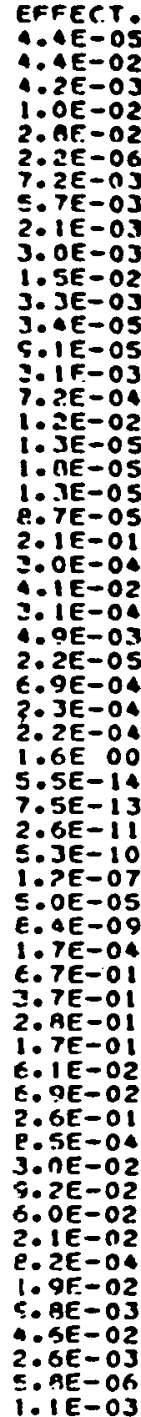 \\
\hline
\end{tabular}

LUNGS

5. OE-OS

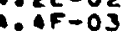

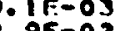

$2.96-02$
$4.6 E-07$

2: $1 E-04$

5. $2 E-06$

I. IE-0S

2. $B F-O B$

7. $0 \mathrm{E}-08$

$1.2 E-07$

5. $9 E-09$

A: $2 E-O 6$

2. $7 E-06$

$1.6 E-06$

3. $O E-02$

5. $7 E-05$

$9.6 E-05$

$7.3 E-05$

$3.9 E-0 B$

3.

$9.1 E-06$

$3.2 \mathrm{E}-3 \mathrm{i}$

$8 E-16$
$0 \mathrm{CE}-14$

2. $6 E-11$

G. 1 E-06

2.:

$\therefore$. $6 E-01$

$6 E-02$
$0.0 E-02$

$1.2 E-01$

2. $8 E-02$

政

Z.TE-05

$3 E-04$
$35 E-03$

i. $9 E-03$

$4.2 E-04$
S. $3 E-07$

3. $7 E-04$

3. $A E-05$

C. $2 E-09$
S. $6 E-O S$

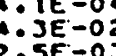

IE -0

: AE -02

IIE -03

$\because 0 E-0.3$
$8.3 E-0.03$

:IE -03

1. JE $=03$

$1.55-03$
$\because .3 E-03$

$2.6 E-04$

$2: 2 E=04$

$2.5 E-04$

1:9E-04

3. OE-O2

1: $1 E-02$

1.7E -03

3.TE-04

$5.9 F-03$

$2.9 E-03$
$3.2 E-03$

2. ᄀE -01

i. $2 E-11$

$\because 3 E-10$

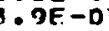

$6.9 E-54$

$3.6 E-53$

:.OE

i.OE 102

$\because 8 E-02$

1. $9 E=02$

$6 . O E-03$

$7.4 E-03$
$6.6 E-03$

$3.5 E-C 3$
$7.5 E-C .5$

$3 . A E-C 3$

$4.5 E-c$

1: 1 E $-\mathrm{E}-\mathrm{aJ}$

2. 2 . $9 E-a .35$
S.IE-05 ULI WALL LLI WAL

$\begin{array}{lll}-02 & 3.1 E-02 & 3: 4 E-02\end{array}$

T.6E -03 A. $3 E-03$

$1.7 E-06$ 1.2E-06 4.JE-07

$1: 1 E-02 \quad 3.8 E-02 \quad 9.7 E-02$

$2.2 E-03 \quad 1.1 E-02 \quad 3.2 E-02$

$1.4 E-02$ B. $2 E-02$ 2.4E-OI

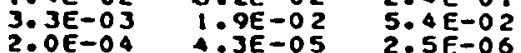

$3.6 E-04 \quad 6.6 E-05 \quad 3.6 E-06$

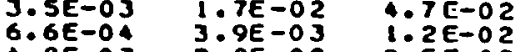

$\begin{array}{lll}8 E-03 & 2.8 E-02 & 8.5 E-02\end{array}$

7.BE-06 5.4E-06 2:1E-0Q

$\begin{array}{lll}4.6 E-06 & 3: A E-06 & 1: 4 E-06 \\ 3 E-04 & 3: 3 E-03 & 6: 6 F-04\end{array}$

3. $D E-02$ 3. JE-02 $4.7 E-02$

$\begin{array}{lll}1.7 E-03 & 5.0 E-04 & 1: 1 E-04 \\ 2.2 E-02 & 6: 2 E-02 & 7: 4 E-02\end{array}$

$3.9 E-03 \quad 2.1 E-02 \quad 5.9 E-04$

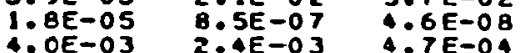

$\because Y E-03 \quad 2 . A E-O 3 \quad 46 \mathrm{E}-04$

8. $6 E-04$ 2.2E-04 2.7E-05

$\begin{array}{lll}7.0 E-23 & 2: 1 E-33 & 3.2 E-34\end{array}$

$\begin{array}{lll}1.6 E-14 & 1: 7 E-14 & 2.0 E-14 \\ 1.7 E-09 & 5.0 E-10 & 7.6 E-11\end{array}$

$8.8 E-00$

I.

$\begin{array}{lll}2.7 E-09 & 2.4 E-09 & 1.9 E-09 \\ 1.3 E-04 & 1.1 E-04 & 1.0 E-04\end{array}$

2.1E-01 $.06 E-01$ 1.0E DO

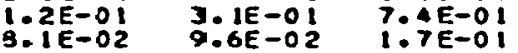

$1.3 E-01$

$5.0 E-02$

3. $1 E=01$

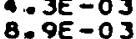

9. $3 E-02$

1. $1 E-01$

?.: $1 E-02$

$1.0 \mathrm{E}-02$

9. $0 E-03$

1. $1 E-02$

$3.0 E-03$
$1.7 E-0.06$

T: OE -03

5.
$5: A E-02$
$6,0 E-02$

$6.5 E-02$

1. $3 E-02$

B.AE-OB
I. IE-OL
ADRENAL

$2.2 E-05$
$5: 2 E-02$
$1.2 E-03$

$3.3 E-02$
$5: 5 E-07$
$3: 5 E$

. $5 E-0$

$3.9 E-04$

$1: 7 \in-05$

1.

. $8 E-0$ B

:OE-O7

. $8 E-05$

C. $2 E-09$
$1.2 E-06$

:3E-07

$2.6 E-06$

.OE-O2

$5.8 E-05$

作

?.3E-05

$4.9 E-08$
$4.9 E-05$

3.4E-DS

2.TE-D1

.2E-34

1.6E-

2. TE-I1

$6.8 E-06$

1.: 0 E-O4

r.6E-01

$8.0 E-02$
$1.00-02$

$1.2 E-01$
$\because B E-C .25$

. $2 E-0$

$2.5 E-02$

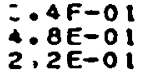

$1.96-01$

1:3E-0?

3. $7 \mathrm{E}-\mathrm{O} 2$

$4.6 \mathrm{E}-0 \mathrm{~B}$
$9.0 \mathrm{E}-\mathrm{O} 3$

$4.5 E-C A$

$2.6 E-03$

IAE-OG

3. $7 E-0.6$

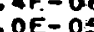

BL FALL KIONEYS $5.1 E-02 \quad 5.2 E-02$

1.. $2 E-02$ ? $.2 E-02$

1. $3.3 E-02$

. 3E-nJ 5.2E-04

$1.2 E-04 \quad 4.4 E-05$

$1.0 E-04$

$2.8 E-08 \quad 1.4 E-05$

$9.8 E-08$ 2. $7 E-07$

1. BE-04 $7.0 E-0 S$

$\because 2 E-06 \quad 4.2 E-06$

$\begin{array}{ll}1: 2 E-06 & 4.2 E=06 \\ 9: 0 E-07 & 8.6 E=07\end{array}$

$6.9 E-07$
$2.3 E=-06$

3. $O E-02$

$5.8 E-05$
$7.1 E \rightarrow 03$

1: OEE-OA

$1.6 E-05$
$5.0 E-05$
$3.0 E-O 5$

$3: 0 E-O 2$

8. $6 E-06$

$3.2 E-34$

1.: $6 \mathrm{E}-16$

$2: 6 E-11$

$2.3 E-08$
$B, B E-06$

$1.96-09$

1:GE-OI

$3.7 E-02$
$3.0 E-O 2$

1.2E-OI

$1.6 E-02$
$3.6 E-03$

.1E-C5 $05 E-0 S$

G. IE-

$2: 3 E-03$
$2: 3 E-03$

$1: 2 E-04$
$1 E-05$

$3: O E-O S$

E. SE

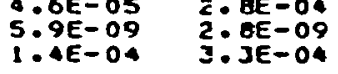

LIVER

$2.1 E-05$
$: B E-02$

$A E-03$

उ: $10=02$

3. $9 E-04$

$6.2 E-04$

$6.7 E-05$
O. OF 05

2E-O4

$9.7 E-08$

$1.7 E-07$

$3.9 E-05$

3. $1 E-02$

$3.2 E-06$

$1.7 E-06$
$9.8 E-06$

$1.4 E 00$

$4.5 E-06$

$6.55-01$

2.1E-0S

$5.6 E-05$

$1.5 E-07$

$4.1 E-03$

$2.3 E-04$

B. OE 00

3. $6 E-38$

$4.7 E-13$

3. $3 E-11$

1. $2 E=05$

6. $2 E-10$

1.3E-01

6. $2 E-02$

$1.2 E-01$

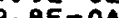

2. $5 E-05$

$1: 4 E-04$

2. $5 E-03$

. $9 E-03$

列

$4.6 E=06$

1:2E-05

$1.2 E-04$

B. $1 E-09$

$8.6 E-05$

$-05$

$3.8 E-08$
$3.0 E-05$

$3.2 E-05$

$1: 4 E-000$

$6.0 E-16$

8.2E-14

$6: 1 E-08$

3. $4 E-06$

$4.6 E-10$
5. $3 E-06$

$1.3 E-01$

6.1E-02

$\because \because 2 E-01$

$6.8 E-02$

$1.2 E$
5.00
3

3. $5 E=03$

$1.5 E-02$

3. $5 E-03$

$3.6 E-06$

. AE-05

$8: \theta E-05$

$5.5 E-09$
$2.2 F-04$

gREAST

$0.1 E-D S$
$\because B E-O Z$

a

3. $1 E-02$

उ. $7 \in=0 ?$

6.6E-0.

$: 1 E-05$
$.6 E-0 S$

3. $3 E-0 S$

$2.8 E-08$
$0.6 E-08$

1.2E-O?

$6.9 E-05$

8. $2 E-06$

1. $3 E-07$

1. $3 E-06$

3. $0 E-02$

$5.8 E-05$
$6.9 E-03$

i. 0 E -04 
INGESTION DOSES AT I YGAR IREMIMICTRCURIEI

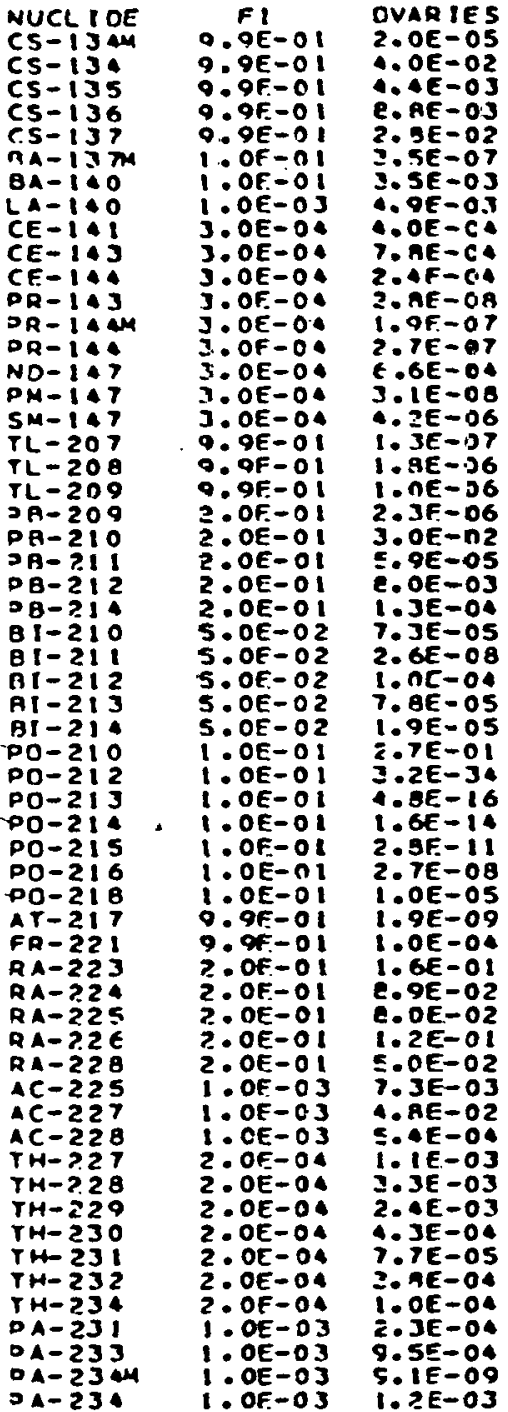

. AE-OS $2: 1 E-05$

$\because 2 E-02$

. उF-06

- SE-O

B. IE-O

are-os

AE-OS

. AE-O

$1.0 E-06$

$7.00-05$

R. 3E-0

$0.2 E=06$
$2.0 E$

$1.2 E-05$

2... $E-06$

3. OE=

3:OE-03

1.SE

3. उE-07

2. $\begin{aligned} & 2 \varepsilon-04 \\ & 1: 4 E-04\end{aligned}$

5. $8 E-05$

3. $2 E-34$

A. $2 E-16$

3. IE-1

2. $2 E-08$

3. $3 E-09$

$1.1 E-04$

$1.6 E-01$
$8.6 E-02$

. OE-O2

\begin{tabular}{l}
$1.2 E-01$ \\
\hdashline $.0 E-02$
\end{tabular}

0

2. $8 E-05$

$1.9 E-04$

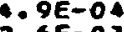

$1.9 E-03$

(

$3.7 E-04$

$1.2 E-05$

1: $1 E-04$

$3.8 E-08$
$3.9 E-04$
2:TE-OZ

R MAR

FNOOST SKIN

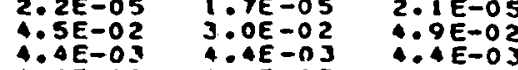

$6.0 E-03$

$1: 0 E-0$
$1: 0 E$

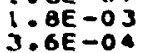

S

5. $9 E-05$

$3: 0 E-06$

$5.6 E-08$
$7.2 E-05$

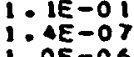

$3.3 E-06$
$3: 0 E-05$

$1.5 E 00$

6. $2 E-01$

$6.1 E-04$
$6.7 E-04$

$1.6 E-08$

1.SE

$1,1 \in-05$
$1,4 \in-0$

$3.0 E-49$
$1: 3 E-1$ is

$1: 3 E-15$
$1: 6 E-13$

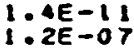

$3.9 E=06$
$1.7 E=09$

2. TE-OS

1.2

S.IE OO

2.1E

2.SE -OI

I:A E

2.: XE-OI

$1.1 E 00$

$7.65-06$

$2.2 E=01$
$6.7 E-05$

B. IE 01

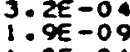

I. OE -04

3: 1 :

$2.7 F-04$

1. $2 E-05$

$1.6 E-05$

$3.0 \mathrm{~F}-\mathrm{OA}$

S. $6 E-08$

$6.2 E-0$

$\because: 3 E-06$
$i=07$

$1.2 E-06$

7.6E-0?

3:OE-O2

5. TE-OS

i: $3 E-05$

$1.7 E-08$
$3.5 E-05$

1 O OE-OS

2. $7 E-0$

$1.6 E-14$

2.6E-11

$5.8 E-06$

$1.0 \mathrm{~F}-04$

$1.66-01$
$8.6 \mathrm{E}-02$

$8.0 \mathrm{~F}-\mathrm{O}$

$1.2 E-01$
$4 . B E=02$

$47 E-04$

$8.3 E-06$

$\because A E-04$

$2.6 E-03$
$1.9 E-0$

C. $2 E-04$

3. 7 F-O

3. $3 F-06$

$6.9 E-05$
B.9E-07 6. 65

$9.7 F-11$
$1.8 E-06$
SPLEEN

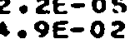

$4.4 E-03$
$1.1 E-02$

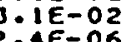

政

$6.6 E-04$
$6.2 E-05$

$8.3 E-05$

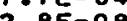

$2.2 E-07$
$4.2 E-07$

$5.8 E-07$
$5.4 E-05$

$7: 5 E-09$

1.:AE-OO

$6.7 E-06$

$2,1 E-07$

: $2 E-06$

$1.8 E-03$
$8.0 E-05$

$\begin{array}{r}3: 3 E-05 \\ \hdashline: B E-07\end{array}$

$6.9 E-05$

8.

1. $4 E$ ol

$6.6 E-15$
$8.2 E-13$

$7.4 E-11$

$6.0 \varepsilon=07$

$1: 1 E-09$

$1.3 E-01$
$6.1 E-02$

$1.2 E-01$
$A B E-02$

$7.1 E-04$

P. $2 E-06$

A.SE-OA
$2.5 E-03$

$1.9 E-03$

3. TE-O6

3. $6 E-04$

$9: 76-06$

$1: 5 \varepsilon-05$

B.
$2 E-05$
$2.2 E-0 B$

2. $6 E-04$
TESTES

$\because 1 E-02$

SE-O3

$2.8 E-02$

: $9 E-04$

:SE-04

$5.7 E-05$
$2.6 E-05$

2.6

1. $2 E-0 B$

$2.5 E-08$
S. $0 E-05$

?. $2 E-09$

: $3 E-07$

.. AE-OT

2. $3 E-06$

3. OE- 02

. $8 E-03$

9. $2 E-05$
$3 E-05$

B. SE-09

6. SE-06

2: $1 E-06$

.2E-34

$1.6 E-14$

2. $2 E-0 B$

$1: 6 E-06$

$1.0 E-04$

$1.6 E-01$
$8.6 E-02$
$1.6 E-02$

B. OE-O2

$1.2 E-01$
$4.0 E-02$

政

$8 E-02$
$30 E-05$

$4 . \theta E-04$

$1.9 E-03$

:9E-06

3. $T E-04$

Q. $2 E-06$

(1)

$7: Y E-10$
$8.7 E-05$
$.2 E-02$
$1 E-03$

$1 \cdot 4 E-02$

IE-O

$1.7 \varepsilon-04$

$6.4 E-05 \quad 2.4 E=05$

3E-06 $1.6 E-06$

3

. $7 E-O B \quad 5.4 E-O 9$

3. $9 E-08 \quad 1: 3 E-08$

.7E-09 5.6E-OO

A. $2 E-06$

.1E-07 $\because 9 E-07$

$2 E-07$
$3 E-06$

3.0E-02

S.7E-0S

.1E-0S 9.0E-0S

.2E-OB $9.6 E-09$

. $1 E-05 \quad 2.8 E-05$

S. $0 E-06$

$2 E-30$

$1.6 E-14$

2. $6 E-11$

2. $1 E=00$

$1: 9 E-O 9$

OE

$8.66-0.02$
$0.0 E-0$

$1.2 E-01$

1. $9 E-04$

$7.9 E-06$

$1: 1 E-05$

SE-0J

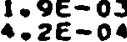

$1.2 E=04$
$3.3 E=0$.

3. $T E=04$

3. $2 E-05$

$5.2 E-06$
$1.7 E-09$

2. $3 E-05$

1.6e-14

2: $1 E=08$

S: $9 E-06$

$100 E-04$

8.6E-02

B. $0 E-02$

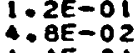

1.AE

$6.4 E-06$
$3.5 E-06$

$4.3 E=04$
$2.5 E=03$

$1: 9 E-03$

3. 4 - 08

3. TE 04

3: $0 E-06$

$1.8 E-06$
$1.1 E-10$

UTERUS

30
$0.06-05$

$4.4 E-03$

B $B E-O 2$

I.AE-O3

2. $3 E-03$

3. $2 E-04$

$2 . B E-O B$

2.TE-07

$2.5 E-0$.

: $2 E-06$

i: $9 E-06$

$1.1 E-06$

3. $0 E-02$

$7.3 E-0 J$

$7.3 E-05$

3. $1 E-08$

8
$7.2 E-05$

3. $4 E-06 \quad 1.0 E-0 S$

2.7E-34 $3: 2 E-34$

$0.8 E-16$
$1.0 E E-16$

$2.8 E \rightarrow 11$

$2.4 E-08$
$9.4 E-06$

1. $9 E=09$

$1.6 E=01$
$B, 7 E=02$

OOE-O2

$1.2 E-01$
$4,9 E-02$

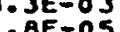

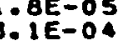

$6.7 E-04$

$2.1 E-03$

1.BE-OS

3.TE-OA

I:OE-O4

$3.8 E-04$
$5.8 E-09$ 
INGESTION DOSES AT I YEAR (REA ALHA

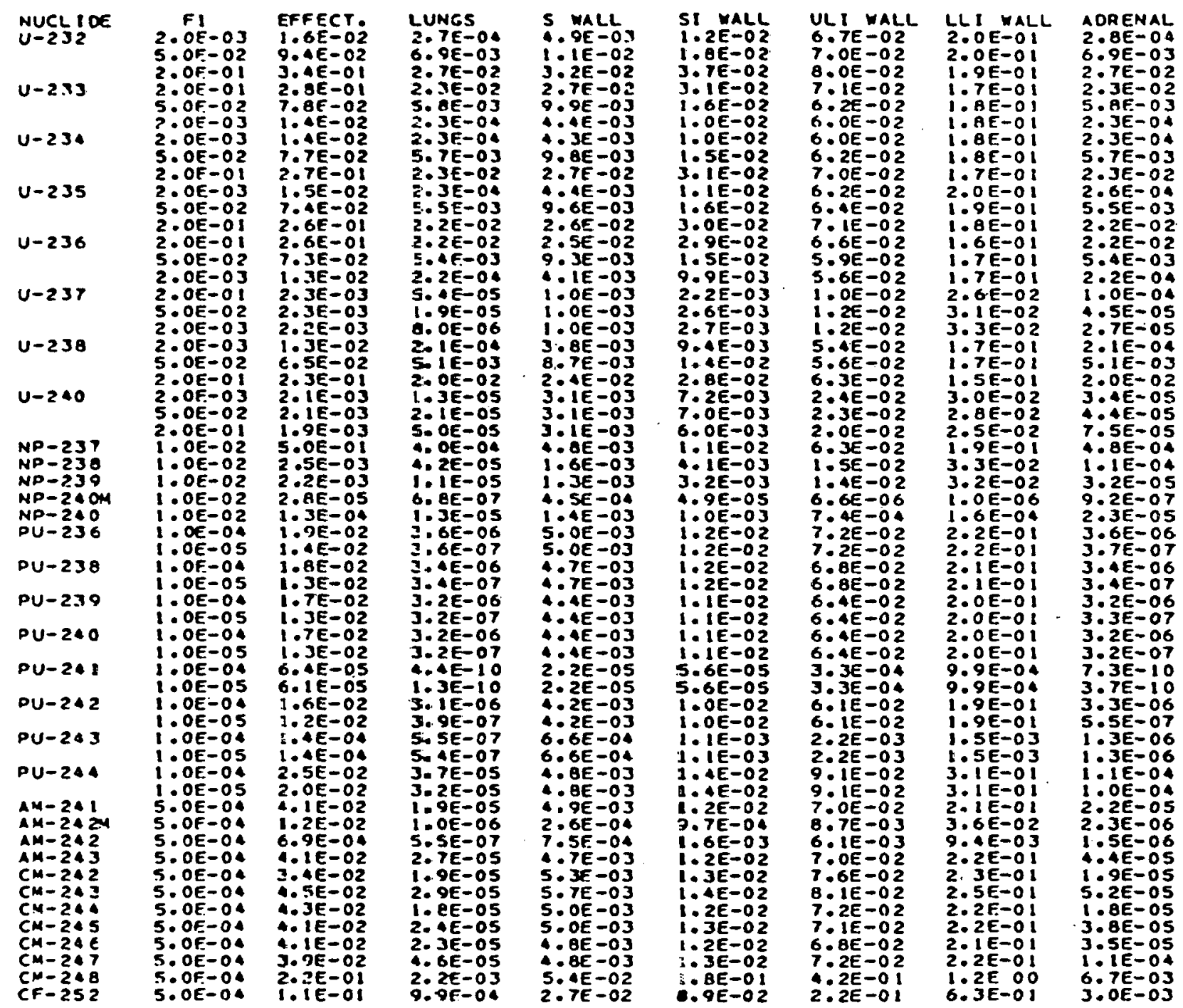

BL $W A L L$ $6.9 \mathrm{E}-03$

$2.7 E-02$
$2.3 E-02$

S. $\theta \varepsilon-03$

(2)

5. $\mathrm{BE}-0.3$

(3E-02

$4: 7 E-04$
$.7 E-03$

政

2.2E-02

政

2. OE-OA

$1: 9 E-04$

2. $2 E-04$

$5.1 E-03$

$1: 1 E-04$

उE-O4

3. $7 E-04$

3. $7 E-O 4$

$3.66-07$

3. $9 E-06$

:PE-0\%

3. $5 E-06$

3: $1 E-07$

S.DE-07

: AE-06

ב: $3 E-09$

כ.: $0 E-09$

: $3 E-06$

BE-06

7. :E-O4

7: $\begin{aligned} & 1 E-04 \\ & \text { G }\end{aligned}$

1. BE-0S

2. $0 E-06$

1. $9 E-05$

1. SE-OS

$1.6 E-04$

\begin{tabular}{l}
$1.3 E-04$ \\
\hdashline $.3 E-04$
\end{tabular}

3. वE-02
1.2E-02 2.1E-0A 2.8E-OA

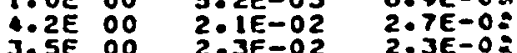

3. BE-OL 5.BE-O3 5.8E-OJ

3. $5 E-02$ 2.3F- 04 2.3E-0

B.7E-01 S.7E-OJ 5.7F-0J

3.5E DO $2.3 E-02$ 2.JE-0?

$\begin{array}{lll}3.2 E-02 & 2.2 E-04 & 3.0 E-04 \\ 8.1 E-01 & 1.1 E-03 & 5.6 E-0 J \\ 1.6 E-02 & 2.2 E-02\end{array}$

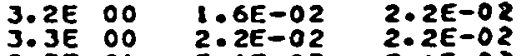

$\begin{array}{lll}\text { 8. } 3 E-01 & 5.4 E-03 & 5.4 E-03 \\ 3.3 E-02 & 2.2 E-04 & 2.2 E-04\end{array}$

$\begin{array}{lll}3.7 E-03 & 2.2 E-05 & 1.0 E-04 \\ 9 & 0.02 & \end{array}$

1.1E-04 $\because .9 E-05$ 6.TE-05

3.1E-02 1.6E-04 2.1E-04

$3.1 E 00 \quad 1.5 E-02$ 2.0E-02

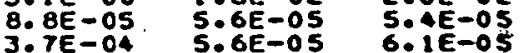

$\begin{array}{lll}3.3 E-03 & 5.9 E-05 & 8.5 E-05 \\ 1.0 E-04 & 2.3 E-00 & 3.7 E-04\end{array}$

2. $3 E-04 \quad 4.3 E-04 \quad 1.8 E-04$

$\begin{array}{lll}7.8 E-05 & 1.6 E-04 & 6.4 E-05 \\ 1.4 E-06 & 8.4 E-07 & 6.1 E-07\end{array}$

$\begin{array}{lll}5.1 E-08 & 2.5 E-02 & 4.0 E-06 \\ 4: 6 E-0 B & 2.5 E-03 & 7.8 E-0 \%\end{array}$

$\begin{array}{lll}3.4 E-06 & 2.6 E-02 & 3.7 E-06 \\ 3.6 E-07 & 2.6 E-03 & 6.96 E-07\end{array}$

3.2E-06 $2: 4 E-02 \quad 3: 3 E-06$

$2,4 E-03$

3.5E-OT $2.4 E-03$

$1.3 E-09 \quad 1: S E-05$

$\begin{array}{ll}1.0 E-09 & 1: 5 E-26 \\ 3.6 E-06 & 2: 3 E-02\end{array}$

9. $3 E-07 \quad 2.3 E-03$

2.6E

2. $4 E-04 \quad 2.3 E-02$

$3: 1 E-05$ 1:JE-OI

$4.8 E-06 \quad 4.4 E-02$

$3: 3 E-06$
$7: 1: 1 E-04$
0

9.1E-02

$1.8 E-05$ I:AE-0!

$\begin{array}{ll}7.2 E-05 \\ 5.7 E-05 & 1: 3 E-01 \\ 3 E-01 & 0.5\end{array}$

2. $5 E-02 \quad \because B E E-O 1$

\begin{tabular}{ll}
$3.8 E-07$ \\
\hline $5 E-06$
\end{tabular}

$3.5 E-06$
$6.7 E-0 ?$

$1.3 E-09$

$1.0 E=09$

1. $3 E-06$

2.2E-06

2. $7 E-04$

2. $7 E-O A$

3. उE-0S

3. 4 E-06

i: 9E-OS

8.7E-OS

$80 \theta E-05$

6. $7 E-05$

6. $2 E-05$

i. $6 E-02$ 
INGESTION DOSES AT I YEAR (REMUMICROCURIE)

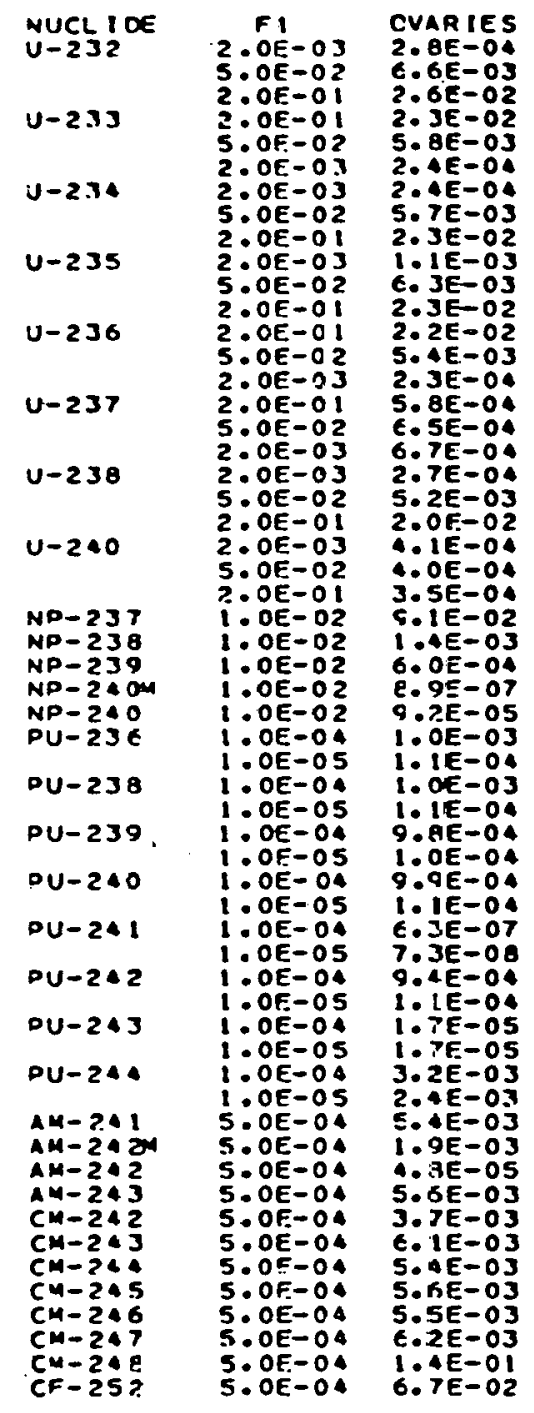

panCREAS BRATN $\begin{array}{ll}\text { 2. OE-OA } & 2.0 E-04 \\ \text { 6E-OS } & 6.0 E-0 J\end{array}$

R MAR 2. TE-O2 . BE-OJ $5.3 E-0:$ $2.3 E-O A$
$5.7 E=03$

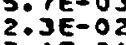
3. IE-OA

$5.6 E-03$

$2.2 E-02$
$2.2 E-02$

SE-O 3

2. 2E-0

- YE-OS

. SE-05

2. $1 \mathrm{E}-04$

$2.0 E-02$

8. $3 E-05$

1E-OS

$4.6 E-04$

2. $2 E-04$

6. JE-06

3. TE-OS

3. ᄀE-O6

$3.5 E-06$
$4.8 E-07$

3. $2 E=06$

3. OE-O 3 -OS

$4.6 E-07$

OEE

3. $7 E-06$

1. $0 E-06$

5. BE-O6

5. 8E-06

$2.3 E-02$

5. BE -03

$2.3 E=04$

2. TE -03

$2.2 E-04$
$5.5 E-03$

$2 \cdot 2 E-02$

2. 2E-O2

2. $2 E=04$

1. JE $=05$

2. OE -0 .

S. IE -03

7. TE-07

. OE -05
O. OS

$3.4 E=04$
$4.5 E-06$

$1.7 E=06$
$1.2 E=08$

3. $6=-06$

$3 \cdot 6=-06$

3. $\begin{aligned} & \text { 3. } \\ & 3.06\end{aligned}-07$

$3.2 E-06$
$3.2 E-07$

$3.2 E-07$
$3 \cdot 2 E-06$

3.2E-07

2. $9 E-11$

3. CE $=07$

3. $0 E-09$

2E-05 1.TE-05

$\begin{array}{ll}4.8 E-06 & 3.6 E-07 \\ 5.3 E-06 & .6 E-08\end{array}$

.

i. 9 E-OS

9.6E-OS

1. $9 E=05$

$1 \cdot 9 E-0.5$
$2 \cdot J E-0.5$

$1 . B E-05$

S. TE-OS $1 . R E-05$

$\begin{array}{ll}1 . A E-02 & 5.3 E-04 \\ 6.5 E-0 J & 2.3 E-O A\end{array}$

1. 1E-0J $1.5 E-02$

SरIN

$\begin{array}{ll}1.1 E-01 & 1.5 E\end{array}$

1. $9 E-02$

$1.0 E$ OD

$1.0 E-02$
1.06

2. $2.5 E-03$

1.

$1.9 E-02$
$7.6 E-02$

$6 E-02$
$6 E-04$

$7.5 E-02$

$7.2 E-02$

OBE-O

$2.0 E-01$
$9.5 E-01$

2. 3E-O $3 E-01$

9. $3 E-03$

I. $6 E=03$

$1.2 E=04$
$: 3 E=03$

1. IE-O

$3.3 E-0$
$3 E-0 S$

$1.3 E-04$

Q. $3 E$ OO

8 . $3 E-04$

$3.6 E-04$

1. $1 E-05$

$9 \cdot 3 E-02$
$9.3 E-03$

9.5E-02

$9.5 E-03$

$9.0 E-03$

. OE- 02

S. TE-OS

5. $7 E-06$

$8.5 E=03$

2. IE-06

$2.1 E-06$

$8.5 E-03$

$0.8 E-01$
$1.6 E-01$

1. OE $1 E-03$

$\therefore 6 E-01$

$3.4 E-O$
S. $0 E-01$

$5.0 E-0$

- RE-OI

$1.7 E-01$
$\therefore E-01$

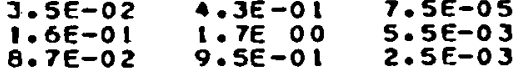

$1.6 E-01$
SPLEEN $2 \cdot 1 E-04$

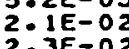

$5.8 E-0 J$

$2 \cdot 3 E-04$

5: $7 E-04$

5. TE-OJ

$5: 6 E-03$

$2.2 E-02$

2. $2 E-025$

$2.2 E-04$

$6.7 E-05$

S. $4 E-05$

5.: $1 E-03$

2.0E-02

$6.7 E-05$

3 . $5 E=04$

$1: 7 E-04$

\begin{tabular}{l}
$5.7 E-05$ \\
$3.5 E-00$ \\
\hline
\end{tabular}

$3.5 E-06$
$6.6 E-05$

$6.6 E-05$
$4.7 E-00$

$3.4 E-06$
$3.7 E-0$

$3.7 E-07$
$3.2 E-06$

3.5E-0?

$3.2 E-06$
$3.5 \mathrm{SE}-07$

1.0 0 - 09

$3.5 E-06$

$8.0 E-07$

$3.5 E-06$
$3.5 E-06$

I:OE-OA

$2.8 E-05$

$3.3 E-06$
$3.3 E-006$

S.
$1.9 E-05$

$1: 9 E-05$

S.6E-OS

:IE-OS

$1.1 E-02$
5. $1 \mathrm{OE}-03$
TESTES

2. $1 E-02$

$2.3 E-02$

2. $3 E-0.4$

2. $3 E-0$

5. $7 E-03$

2. $\mathrm{BE}-04$

5. 5E 03

2. $2 E-02$

$5 . A E-03$

2.2E-O4

$5.3 E-05$

2. $2 E-04$

$5.1 E-03$

2. $0 E-02$

$3.2 E-05$

3. TE-02

$1.2 E-04$

5. $8 E-0.08$

1: $0 E-03$

$1: 0 E-03$

$1.0 E-03$

$1: 0 E-04$
$9.8 E-04$

9. $\mathrm{BE}-05$

9.

-07

6. $3 E-08$

9. AE-0S

6. 1E-O

i: $A E-03$

5. $3 E-04$

1: $8 E-03$

$1.4 E-05$

5.0

3. $7 \varepsilon=03$

5. $4 E-0 J$

5. $1 E-03$

5.:2E 03

$5.2 E-02$
$2.6 E-02$
mYuus

2.PE =

. $7 E-02$

$2.3 E-02$
$5.8 E-03$

2. $3 E-04$

5. $7 E-03$

2. $3 E-02$

$5.5 E-03$
$2.2 E-02$

$2.2 E-02$

$2.2 E-04$

I. $2 E-05$

2. $7 E-06$

(2.06

. $0 E-02$

1.:4E-05

4. $2 E-05$

$3.3 E-04$
$: 9 E-05$

A. $1 E-06$

$1: 7 E=07$
$\therefore .4 E=06$

3. $6 E-06$

J. $A E-06$

$3.4 E-07$

3. $2 E-07$

$3.2 E=06$
$3.2 E=07$

2. $9 E-10$

5. SE-11
3. OE-OG

3. $3 E-07$

1. $2 E-07$
$1,2 E-07$

1. BE-05

1. $7 E-05$

3. $6 E-07$

i. $9 E=05$

$1.9 E-05$

1. BE-OS

$1.9 E-05$
$1.9 E-05$

2. $8 E-05$

$1.0 E=03$
$1.0 .0 E-04$

THYROIO UTERUS

$6.9 E-03 \quad 6.9 E-0 J$

.JE-02 2 JE-OZ

$5.8 F-03 \quad 5.8 E-03$

उE-04 $2.3 E=0$

- $7 E-03$ S. $7 E-03$

SE-O $5.6 E-0$.

S. $0 E-03$

(1)

(1)

-2E-05 2.7E-O.

.

(

2.OE-O2

$1: 1 E-05$

M. IE-OS

$3.2 E-04$
$3: 5 E-06$

$1: 9 E-06$

O. $2 E-08$
$1.4 E-06$

3. $6 E-06$

$3.4 E-06$

3. $1 E-06$

3. $2 E=07$

$3.2 E-06$
$3.2 E-07$
$3.0 E-17$

$3.6 E-10$
$3.0 E-11$

3. $0:=06$

$2.2 E=08$

2. $0 E=08$

3.7E-06

$3.7 E-05$
$2.2 E-07$
$G E-07$

6. $5 E-08$

$1.8 E-05$

. $9 E-05$

1: TE-05

2. $1 E-05$

3: TE=D:

2.7E-O

S.1E-0J

2. $0 E-02$

1.9E-:

3.9E-D:

2.SE-OA

$8.8 E-07$
$7.0 E-05$

i.TE-:O

$3.7 E-06$

6.5E=07

S. SE-0 
MAXIMUM ANNUAL INHALATION DOSE

(REMUMICROCOZTIE)

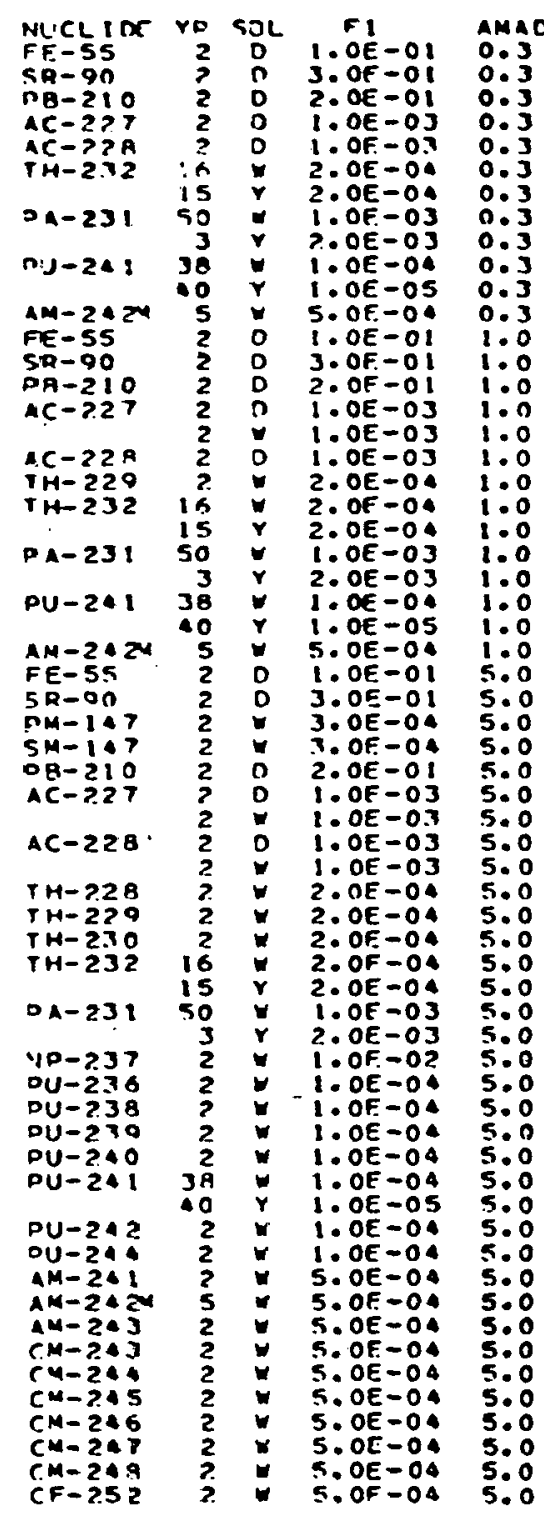

EFFECT: LUMGS

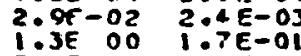
3.AF $02 \quad 3 \quad 2 E-0$. 5. $1 E$ OI $2.3 E-0$ अ.6E भI 1. OE O2 3.Ke D2 Q. 2. $96-01 \quad 7.7 \mathrm{~F}-0 \mathrm{~S}$ AF oi 3 BE-Od $6.9 E-04 \quad 5.3 E-0$ 2:TF-02 $2: 3 E-03$ $3.2 E$
3.06
1.02 7.TF-02 1.TE-OS $\begin{array}{llll}6.4 E & \text { ol } & 8.3 E & 00 \\ 0.06 & 21 & 2.2 E-02\end{array}$ 3.AE OI 5:BE OI 6. TE DI $5.6 E$ O2 .6E- Ol h.9E-o ..2E Di $2: 5 E-0$. $3.3 F-0$ 2 $2.8 \mathrm{BF}-03$ . TE-03 $2.3 E-O$ $1.4 E$ DO 1. $8 F-0$ 3. $3 E$ O2 $2.1 E-02$ 8.3E O1 $2.8 E 0$ $2.1 F-02$ C.2E-O T.OOE DI $1: 5$ E OO $1.3 E$ of $8.5 E-0$ 5.OF O1 2.3E-02 .SE OI $9.5 E-0$ $2.4 E$ ol $2.0 E-02$ $1.2 E$ ol $\because A E-O$ $1.4 E$ Ol $.7 E-0$

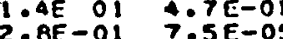

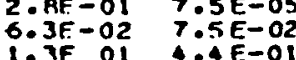
l. $3 E$ Ol $\triangle A \Delta E-O$ 1..3F of $2.7 \mathrm{E}-0$ liAE ol $1.9 F=01$ .SF ol $5.1 F-0$ 1. SE Of $4.9 E-0$ l. $3 E$ ol $4.5 E-0$ $5.3 E$
$2 . A E$

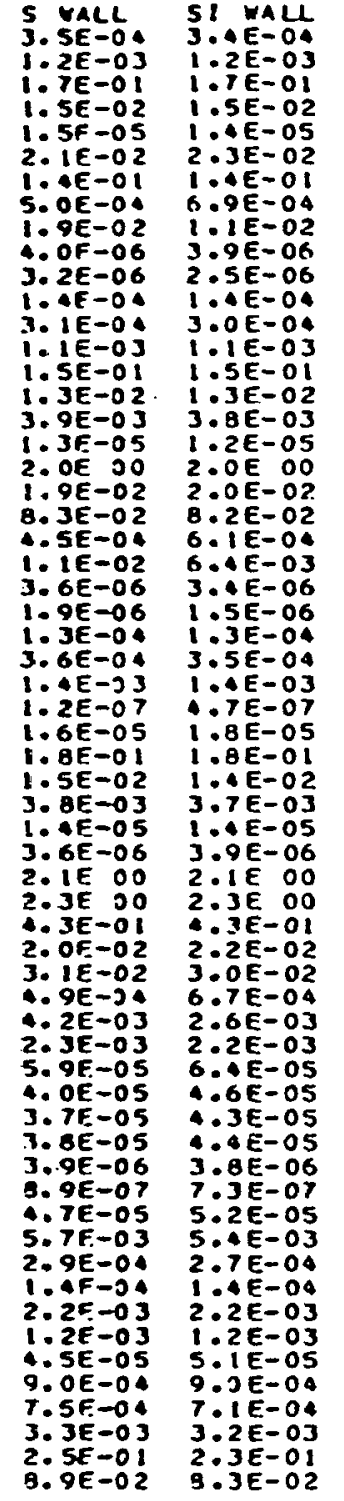

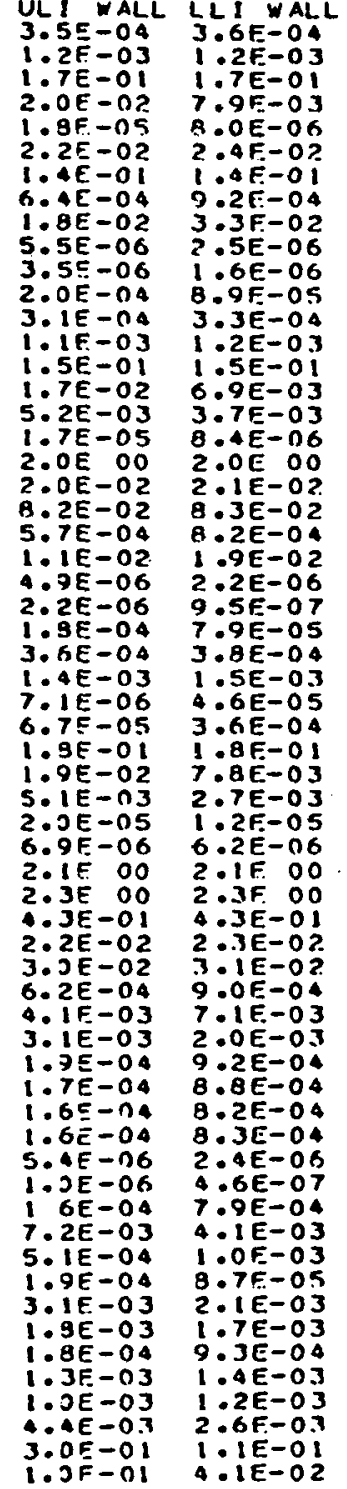

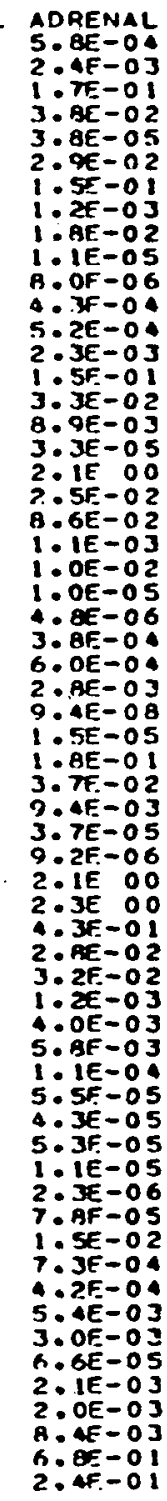

BL BALLL $2 \cdot 4 E-03$ $\therefore 6 E-03$ 5.:1E- 02 $\because \because A E-01$ $8.1 E-03$
$0 . \Delta E-07$ 5.9E-07 3. OE- 05

S. DE- OA

$1: 5 E-01$

i: $5 E-03$

2..6E- 06

$1.9 E-02$
$8: 2 E-02$

$\because 2 E-04$

B.AE-07

$3.6 E-07$
$3.5 E=05$ 6.OE-O $2.8 E=03$ $2.5 E-08$
$1.5 F-05$ $\because A E-03$ $1.3 E-03$
$5.2 E-06$ $1.4 F-06$ 2.1E 00 ‥JE- OI 2. $1 E-02$ $4.6 E-04$ 2. $1 E-0.2$ $4.2 E-05$ 3. $A E-05$ $3 . \triangle E-O S$
3. $\triangle E-O S$ $9.2 E-07$ 1. $8 E-07$ $1: 9 E-03$ $8.2 E-05$
$3.8 E-05$ $6.3 E-09$ 3. $\mathrm{AE}-04$ 2. $9 E-04$ 2. $4 E-04$ $1: 1 E-03$ $7: 4 E-02$
$2.6 E-02$
KIONEYS LIVER 2: $4 E-03 \quad 205 E-03$ 2. $9 E-02$ 1:GE 03 1.SEE-OI I.4F-O l.2E OO Q.OE OA $1: 0 E-02$ $1.2 E-006$
$8.6 E-06$
$3: 9 E-01$ 3. $1 E-06$ 6. $3 E-0$ 5. 2E-04 $1.7 E-03$ 2.3E-03 $2: 3 E-03$ 2.5E-02 $1.4 E$ OJ $2.2 E-0 S \quad 3.7 E-O$ 2:OE-OO $1: 7 \in$ - 0 8. उE-02 $6.8 E-0$ $\begin{array}{ll}7.2 E-04 & 5.4 E-04 \\ 7.2 E-01 & 1.1 E-02\end{array}$ A. 2. $A E-04$ 5.5E OI $2.8 E-03 \quad 2.8 E-03$ $1,5 E-05$ 4. OE OO $2.8 E-02$ 7.: $2 E-03$ $2.5 E-05$
$6.2 E-06$ 2.1E 00 2: $3 E$ O० ‥3E-OI 3.7E OO 3. $1 E-02 \quad 1.5 E-0$ . $45 E-03$ G.2E OI . AE-OS $4.3 E-05$ 3. BE-OS $4.1 E-05$ $8.4 E-06$
$1.6 E-06$
$0.0 E-065$ $6.2 E-05$ $1,1 E-02$ 3. $0 E-04$ $4.4 E-03$
$2.4 E-03$ $1: B E-03$ i. $.6 \mathrm{E}-03$ $6.1 \varepsilon-03$ 5. $5 E-01$
DHEAst 2 iaf -03

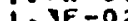
$1.3 F=05$ $1 . A E-01$ $9 A F=$ $\because 2 E-06$ $1.65-04$ $5.2 E-04$ $2.3 E=03$ 1:SE-02 2: $\begin{aligned} & 1 E-05 \\ & 1 E \\ & \text { I }\end{aligned}$ B. $4 E-02$ B. $4 E-04$ 8. $3 E-06$ 2.:OE $=06$ (1) $2.8 E-03$
$4.5 E-0 B$ I. $5 E-05$ $1: B E-01$

$1: 3 E-02$
$3: 4 F-03$ 3. $25-05$ 2. IE 00 ..3E-O $2.4 E=02$ $3.1 F-02$ $9.2 E-0$ $2: 1 E-03$ $6.0 \mathrm{E}=05$ 3: $:$ E -05 4.1E-O $\because$ IE -06 $5.00-05$ $5.6 E-03$ 2. $B E=0$. $1.9 E-03$

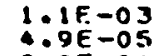
8 .OF -04 $8.0 E=04$
$2.9 E-03$ $2.7 E-01$
$0.5 E-02$ 
MAXIMUM ANAUAL INHALATION DOSE (REMPMICROC̈URIE)

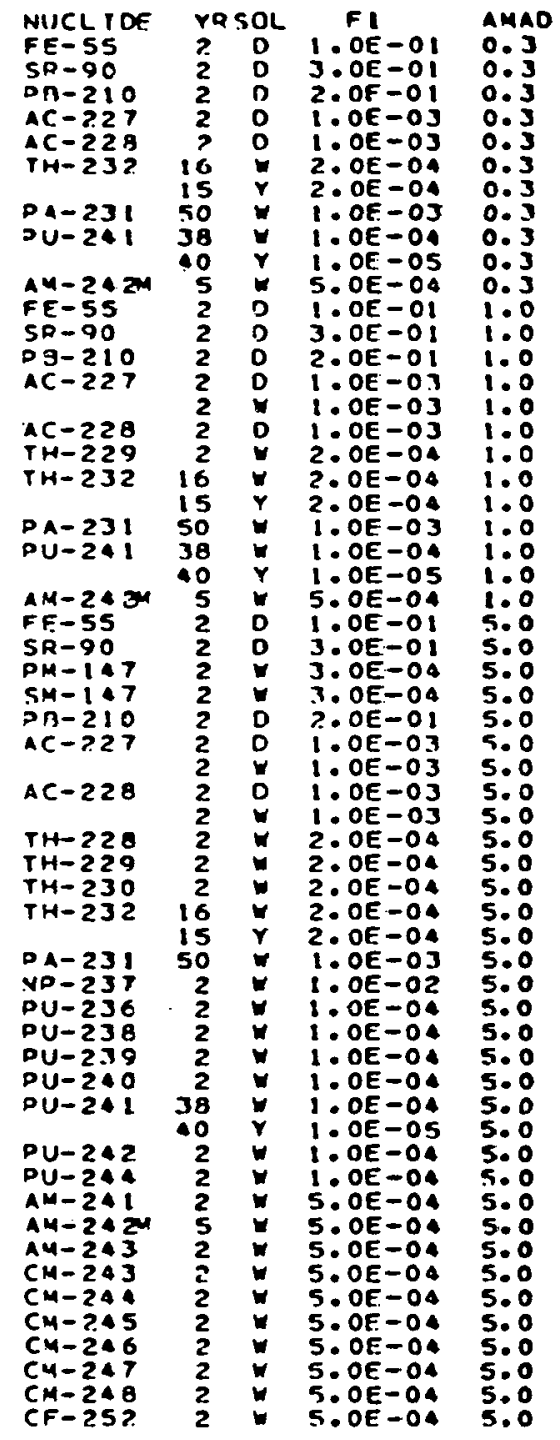

CVARIFS PANCREAC araIh S.ZE-DA $6.0 E-04$ 5. BE-04 . TE-OI I.TF-OI $6 . A F$ OI J.2F-0.2

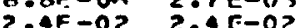
$1 . A F=01$ I.SE-01 $\begin{array}{ll}7 . A E-O A & 9.6 F-D A \\ 7.5 F-02 & 9.3 E-06\end{array}$ .6E-02 $6.8 E-06$ $2.6 E 00 \quad 3.3 E-04$ $2.3 E-03$ 2.3F-03 .5F-OI 1.5F-OI

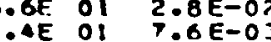
2.8E-06 $2.4 E-05$ 2. IE-02 2.2 E-02 $8.3 E-02$ Q.5E-02 6.RE-OA 7.7E-OA $\begin{array}{ll}6.7 F-02 & 9.3 E-06 \\ 2.8 E-02 & -1 E-06\end{array}$ .je on $2.9 \mathrm{E}-\mathrm{O}$ 5.9F-04 $6.1 E-04$

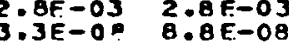
.5F-05 $1.5 \mathrm{~F}-0.5$

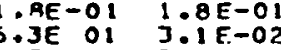
1.5E ol $7.9 E-03$ $\begin{array}{ll}.8 E-06 & 2.7 E=05 \\ 2.3 E-06 & 6.6 \mathrm{E}-06\end{array}$ $2.1 F$ OD $2.1 E$ OD $.3 E-01 \quad 4.3 E-01$ 2.3 3.1

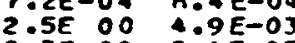
政 2.6E $00 \quad 3.86-05$ 2.6E 000 4:1E-05 $1.4 E-02 \quad 1.9 E-06$ - AE ON $6.1 E-O$ 2.7F. $00 \quad 6.3 \dot{E}-04$ 2.6E 00 3.2E- 04 2. $0 F$ OO $2.6 \mathrm{~F}-03$ 2.AE 00 S.1F-OS 2.7E OO $1.5 \mathrm{E}-03$ 9.9F OO S.OE-OI
I.SE OO I.AF-OI

$1.7 E-01$
$1 E-02$

. 5E-OI

$\because 3 E-06$

$\begin{array}{r}\text { ?. } \\ 1.3 E-06 \\ \hline \text {. } 04\end{array}$

S: $2 E-04$

$1.5 E-01$

1. OE -02

9. JE-06

2.
$8 E O-O 2$

$1.5 \mathrm{E}-\mathrm{O} 3$

$1: 7 E \rightarrow 06$

$1.2 E-04$
$6.0 E-04$

$2: 8 E-03$
$4: 0 E-J B 8$

1. $5 E-05$

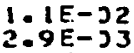

$1,00 E-95$

2. 1150

$3.3 \mathrm{E}-\mathrm{O} 1$
$3 \mathrm{E}-\mathrm{O}$

$3.2 E-82$
$1.6 \mathrm{E}-0.3$

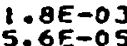

$3.2 E-05$
302

$\because 0 E-C 5$
$\because .2 E-C 6$

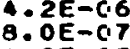

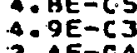

$1.3 \mathrm{E}-\mathrm{a} A$
$1.0 \mathrm{E}-\mathrm{CJ}$

P.: $T E-04$

6.5E-OA

2. 5E-aJ

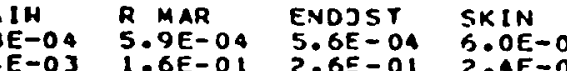

$02 \quad 5.9 E \quad 0.3$

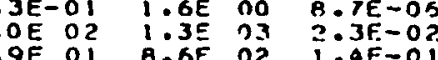

- ?E OI $9.1 E$ OL $\quad 0.8 F-04$

.TE-OI $3.3 E$ OO

$1.9 E-11$

$1.5 E-O i$

$1: 0 E$
$1: 1 E-O L$

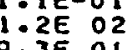

$\because 2$ OI

3.AE-

I:GE-D

IE-O

$8.2 E-03$

?:? O OO

$\begin{array}{lll}4.6 E & O 2 \\ 1.1 E & O 2 & 1 \\ 3.2 E & 01\end{array}$

$1.2 E-01$
$3.0 E-02$

$1.3 E$ O.

$\begin{array}{lll}2.6 E & 01 \\ 1.0 E & 02 \\ 2.0 E & \text { OL }\end{array}$

$2.0 E$ OI

\begin{tabular}{lll}
$3.3 E$ & :O \\
$3.3 E$ & 02 \\
\hline
\end{tabular}

$2.4 E-01203 E-03$

$.1 E$ O3 $7.0 \mathrm{OF}-\mathrm{O}$

$1.3 E$ O3 $2.3 E-03$

IAE OO $7.6 E-06$

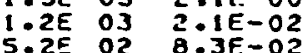

.IE $026.1 E-04$

$4.8 E$
$2.0 E$

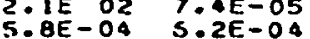

$3.0 E-01$ 3.AE-O3

9.6501 l.5E-O5

$1.4 E$ OI $1.8 F-01$

:TE O3

$7.8 E-03$
$3.1 E-03$

1.SE 00 B $8.5 E-06$

$1.6 \mathrm{E}$ O3

$1.7 E \quad 03 \quad 2-1 E$ D

$3.2502 \quad 4.3 E-01$

1.3E OJ 2.3E-02

IE OI B.QE OZ

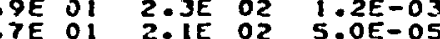

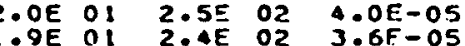

$1.9 E$ O

B.OE-02

:AE OI

$2.0 E$ OI

$1: 9 \mathrm{E}$ OI

$\begin{array}{ll}2.1 E & \text { OI } \\ 2.0 E & \text { OI } \\ 2.0 E & \text { OI }\end{array}$

2 :OE :

$10 B E$ OI

$\begin{array}{lll}2.4 E & O 2 & 3.6 F-05 \\ 2.4 E & 02 & 3.8 E-05\end{array}$

$5.2 E$ DO

यद $4.3 E-07$

. 02 3.7F-03

2.35 O2 $\mathrm{g}: \mathrm{SE}-04$

$2.4 E$ O2 $1.1 E-03$

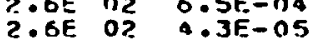

2.SE Oर $4.5 E-04$

-SE 02 5.3E-0Q

2.3E O2 $1: A E-03$

$\begin{array}{ll}3.3 E-0.1 \\ 9.2 E-0.2 & 3: 3 F\end{array}$
SPLEEN TESTES

$\begin{array}{ll}3.1 E-03 & \text { 5. RE-04 } \\ 3.4 F-03 & 2.4 E-0.3\end{array}$

$\begin{array}{ll}1.7 E-01 & 1.7 E-01 \\ 1.0 F-O 2 & 6.5 F-01\end{array}$

OAE-OG

- JE-02 $20.3 E-0$ ?

$\begin{array}{lll}A E-J 1 & 1.4 E-01 \\ 3 E-0.4 & 0.7 E-O G\end{array}$

2. $6 E=06$ 7: $5 E-02$

2.AE - $5 E-05 \quad 4.6 E-05$

$2: 7 E-03 \quad 5: 1 E-01$

$1.5 F-01$ I. $5 E-O 1$

$2.2 E-03$ I. $4 E$ OI

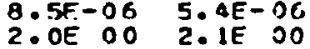

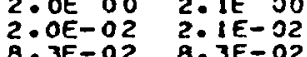

4. $9 \mathrm{E}-04$

$\begin{array}{ll}3 E-06 & 5.7 E-22 \\ 1.5 E-06 & 2.0 B E-02\end{array}$

B. $5 E-05$

5. 0 3E-0O

$2.8 E-03 \quad 2.8 E-5 Z$

$1.5 E-051.5 E-05$

$\begin{array}{lll}1.0 E-01 & 1.8 E-01 \\ 0.9 E-03 & 6.3 E & 01\end{array}$

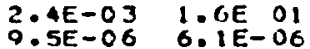

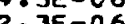

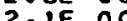

2. उE $: 00$

$1.5 E-06$

2. $3 E$ OO

$\begin{array}{ll}3.2 E-02 \\ 3.15 & 2.3 E-02\end{array}$

$\begin{array}{ll}3.1 E-02 & 3.1 E-02 \\ 6.2 E-04 & 5.2 E-04\end{array}$

$1 \cdot 5 E-03$

$3.9 E-05$

$3.5 \times-05$
$3.7 E-05$

2. SE 00

2. TE 00

2. KE nO

$6.5 E-07 \quad 1: 4 E-02$

$4: 1 E-03$ 2: 4 DE

$1.8 E-04 \quad 2$. $7 E$ OO

$1.4 E-03$
$8.25=04$

2. JE 00

$5 . \mathrm{BF}-04$

2. $35-03$

2. $A E$ OO

2. IE DO

2.7F 00

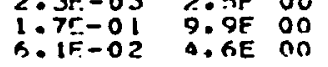

THYMUS THYROIO UTERUS

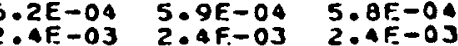
. $7 E-01$ 1:TE-O1 $1: 7 E-01$ $1.0 F-05 \quad 4: 4 E-06 \quad 6.9 F-00$

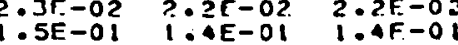

$\begin{array}{rl}2 E-04 & 9.2 E-04 \quad 5: 3 E-04\end{array}$

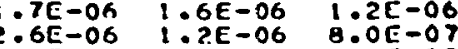

. $2 E-05$ 5. $0 E-05 \quad 5.3 E-05$

2.3E-03 2.3E-03 $2.2 E-04$

..5E-01 $1.5 E-01 \quad 1.5 E-01$

$\begin{array}{lll}7.6 E-03 & 5.9 E-03 & 5.3 E-0 J \\ 2.5 E-03 & 2.0 E-03 & 1: 8 F-03\end{array}$

$2.06-06 \quad 3.9 E-06 \quad 5.6 E-06$

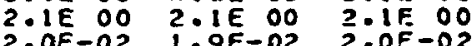

$8.5 E-02$ a: $3 E-02$ R $\quad: 2 E=02$

$1.5 E-06 \quad 1.4 E-06 \quad 1.1 E-06$

$1.5 E-06 \quad 7.0 E-07 \quad \therefore 9 E-07$

G.4E-O4 $6: 1 E-04$ G:OE-OA

$\begin{array}{lll}2.8 E-03 & 2.8 E-03 & 2 . A F-0 J \\ 3.2 E-08 & 2.9 E-0 B & 2.9 E-0 B\end{array}$

. $5 E-05$ 1:5E-05 1:5F-05

$\begin{array}{lll}2 E E-01 & 1.8 E-01 & 1.8 E-01 \\ 8.5 E-03 & 5.0 E-03 & 6.0 E-03\end{array}$

$\begin{array}{lll}2.3 E-03 & 1.8 E-03 & 1: 3 E-03\end{array}$

2.5E-06 1:1E-06 $1: 3 E-06$

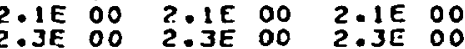

$4.3 E-01$ 4.3E-OL $4.3 E-01$

::E-02 $3: 1 E-02$ 2:1E-02

. IE-04 $9 . n E-04$ S.2E-04

. OE-05 $\quad .1 E-05 \quad 4.4 E-05$

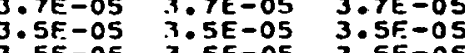

3.5E-05 3.5E-05 3.5E-0S

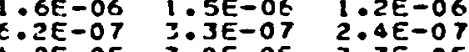

3.7E-03 $2.5 E-03 \quad 2.4 \vec{E}-03$

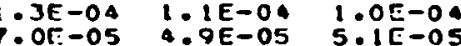

$1 E-03 \quad 8.9 E-04 \quad 8.6 E-04$

.1E-0S $4.0 E-05$ 5.2E -04

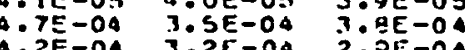

$2.2 E-03 \quad 1.6 E-03 \quad 1.3 E-03$

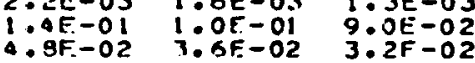


MAXIMUM ANNUAL INGESTION DOSE (REMPMACROCURIE)

\begin{tabular}{|c|c|c|c|}
\hline 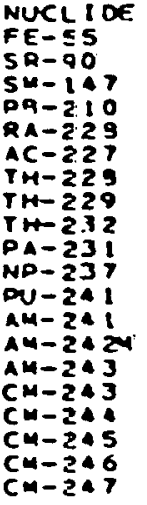 & $\begin{array}{r}Y R \\
2 \\
2 \\
2 \\
2 \\
3 \\
2 \\
2 \\
2 \\
2 \\
16 \\
50 \\
2 \\
32 \\
2 \\
5 \\
2 \\
2 \\
2 \\
2 \\
2\end{array}$ & 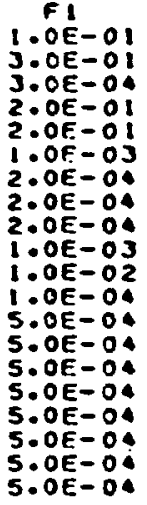 & $\begin{array}{l}\text { EFFECT } \\
1 . A E-00 \\
1.5 E-02 \\
1: 2 E-02 \\
1: 7 E-01 \\
1.7 E-01 \\
6.2 E-01 \\
9.5 E-02 \\
1.1 E-01 \\
7.5 E-02 \\
2.6 E-01 \\
9.7 E-01 \\
2.1 E-01 \\
5.6 E-02 \\
5.0 E-02 \\
5.4 E-02 \\
5.8 E-02 \\
5.7 E-02 \\
5.5 E-02 \\
5.5 E-02 \\
5.0 E-02\end{array}$ \\
\hline
\end{tabular}

\begin{tabular}{|c|c|}
\hline 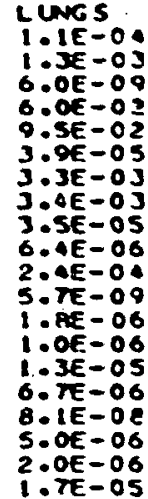 & 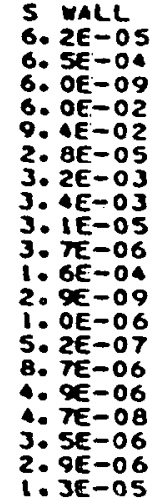 \\
\hline
\end{tabular}

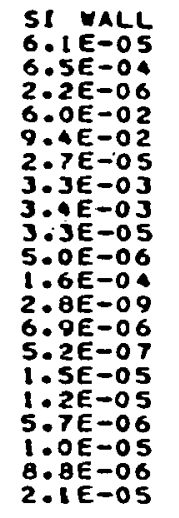

UI WALL
$6.4 E-0 S$
$6.8 E-04$
$5.4 E=05$
$6.0 E-02$
$9.4 E-02$
$4.7 E-05$
$3.5 E-03$
$3.5 E-03$
$3.3 E-05$
$3.7 E-06$
$3.4 E-04$
$41 E-09$
$1.4 E-04$
$7.3 E-07$
$1.5 E-04$
$1.6 E-04$
$1.4 E-04$
$1.4 E-04$
$1.3 E-04$
$1.6 E-04$

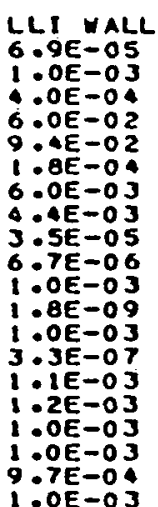

ADRENAL

1. OE $=04$
$1.3 E-03$

BL YALL

$x$ IONEYS

LIV VER

$1.3 E-0.3$
$1.0 E-0.03$

作

$9.5 \mathrm{E}-02$

$6.0 E-09$

$1.3 E-03$

1. JE OO

$9.6 E-02$

S. $3 E-05$

$3.4 E-03$

3. $5 E-05$

5. $9 E-06$

$5 E-03$

$3.4 E=03$

3. $4 E-06$

$3.2 E-04$
$6.3 E-09$

2.1E- 06

- $2 E-04$

6.9E- 10

1. IE-06

$2.0 E-05$

1. IE-OS

$1.4 E-07$

1. $7 E-05$

9.2E-06
$7.9 E=08$

$\begin{array}{ll}1.6 E-06 & 6.8 E-06 \\ 1.2 E-06 & 6.0 E-06 \\ 6.1 E-06 & 2.0 E-05\end{array}$

$\begin{array}{ll}1.6 E-06 & 6.8 E-06 \\ 1.2 E-06 & 6.0 E-06 \\ 6.1 E-06 & .20\end{array}$

$7.3 E=06$

S. OE-O2

$9.4 E-02$

2. $9 E$ E 00

$2.9 E-02$

2E-

$4 E-06$
$4.4 E$

$7.3 E-04$

.2E-OI

2.6E-O

. $6 E-01$

$2.5 E-01$
$2.5 E-01$

2. $3 E-0$ I

BReAst

$0.06-04$
$1.00-03$

$0.0 E-00$
$6.0 \mathrm{E}-08$

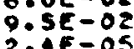

$3.3 E-03$

उ. PE $=03$

$6.9 E-00$

उ.je $=08$

\%OE-O?

$7.5 E-06$

$3 E-06$
$6.8 E-08$

3. $1 E-00$

$3.1 E-00$

... 
MAXINUM ANMUAL INGESTION DOSE (REMIMICROCURIE)

\begin{tabular}{|c|c|c|c|c|}
\hline 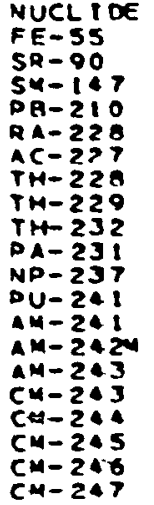 & $\begin{array}{r}r \\
2 \\
2 \\
2 \\
2 \\
3 \\
2 \\
2 \\
2 \\
16 \\
50 \\
2 \\
35 \\
2 \\
5 \\
2 \\
2 \\
2 \\
2 \\
2 \\
2\end{array}$ & 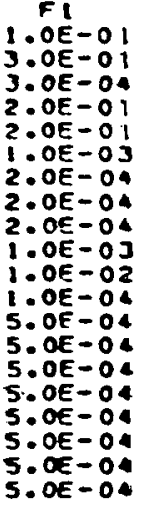 & 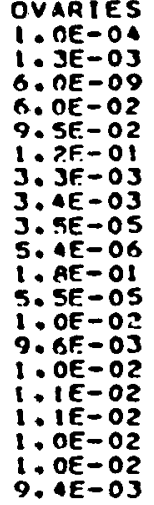 & 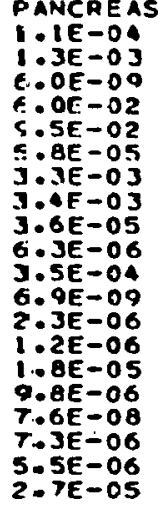 \\
\hline
\end{tabular}

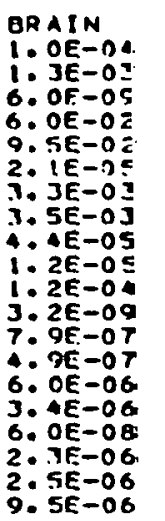

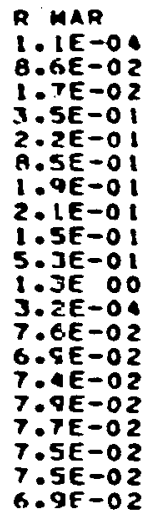

ENDOST

SKIN

1. $\triangle E-O I$

$1.30-03$
$1.15-0,04$

SPLEEN TESTES

THYMUS

THYROID

UTERUS

$2.2 E-01$
$-S E$ OO

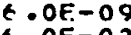

i. $3 E-03$

$1.1 \mathrm{E}=04$

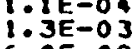

1.OE-0.

S.CE- 09 6. $.0 E-09$ 6.OE-O9

9. 5E-02

..

$6.06-09$

$1.6 \mathrm{E}-\mathrm{O}$

$9.5 F-02$

o.r. 02

1. IF 01

$1.4 E-05$

3. $3 E-03$

$3.4 E-03$

(6E-05

3E-05

.9E 00

1.7E OI

$3.4 E-0.3$

(3)

$1.00-1) 1$

$5.5 F-05$

$9.2 \mathrm{E}-\mathrm{OS}$

1.OE-02

$1.2 E-09$

$3.3 E-03$
$3.4 F-03$

$3 \cdot 9 E=06$

.1E-09 9.3F-10

$0.5 E-0$

B. $6 E-01$

OF -02

3. OE $=0$

$4.9 F-07$
$1.96-07$

$3 \cdot 1 E-07$

-

$1.95-07$

. $9 E-0$

$1.0 F-02 \quad 1.7 E=06$

1.9E-06

$3,4 E-08$

2 . OE -06

$2.0 E-O S$

a.n:-06

B. AE -06

6. OE-OG

1:AE
$7.1 F=06$ 